OAK RIDGE

NATIONAL LABORATORY

MANAGED BY UT-BATTELLE

FOR THE DEPARTMENT OF ENERGY

\title{
AGR-2 Fuel Compacts Information Summary: Prepared for the NRC MELCOR Project
}

\author{
Revision 1
}

John D. Hunn

November 2010

Prepared for the

United States Department of Energy Office of Nuclear Energy under the

Next Generation Nuclear Plant Advanced Gas Reactor Fuel Development Program 


\section{DOCUMENT AVAILABILITY}

Reports produced after January 1, 1996, are generally available free via the U.S. Department of Energy (DOE) Information Bridge.

Web site http://www.osti.gov/bridge

Reports produced before January 1, 1996, may be purchased by members of the public from the following source.

National Technical Information Service

5285 Port Royal Road

Springfield, VA 22161

Telephone 703-605-6000 (1-800-553-6847)

TDD 703-487-4639

Fax 703-605-6900

E-mail info@ntis.fedworld.gov

Web site http://www.ntis.gov/support/ordernowabout.htm

Reports are available to DOE employees, DOE contractors, Energy Technology Data Exchange (ETDE) representatives, and International Nuclear Information System (INIS)

representatives from the following source.

Office of Scientific and Technical Information

P.O. Box 62

Oak Ridge, TN 37831

Telephone 865-576-8401

Fax 865-576-5728

E-mail reports@adonis.osti.gov

Web site http://www.osti.gov/contact.html

This report was prepared as an account of work sponsored by an agency of the United States Government. Neither the United States Government nor any agency thereof, nor any of their employees, makes any warranty, express or implied, or assumes any legal liability or responsibility for the accuracy, completeness, or usefulness of any information, apparatus, product, or process disclosed, or represents that its use would not infringe privately owned rights. Reference herein to any specific commercial product, process, or service by trade name, trademark, manufacturer, or otherwise, does not necessarily constitute or imply its endorsement, recommendation, or favoring by the United States Government or any agency thereof. The views and opinions of authors expressed herein do not necessarily state or reflect those of the United States Government or any agency thereof. 


\section{Table of Contents}

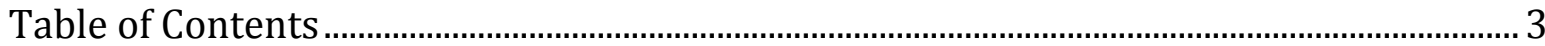

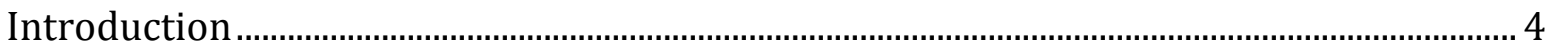

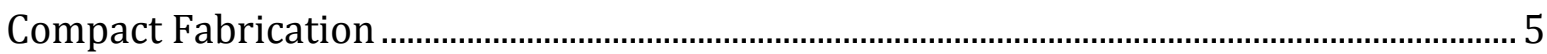

Summary of Particle and Compact Properties ...................................................................... 11

Appendix A : Compact dimensions, mass, matrix density, and packing fraction............ 14

Appendix B : Particle diameter, weight and volume ............................................................ 57

Appendix C : Particle and compact uranium loading ............................................................... 71 


\section{Introduction}

This document was prepared at the request of the NRC to provide summary information on the compacts used in the Department of Energy's Office of Nuclear Energy (DOE-NE) Advanced Gas Reactor Fuel Development and Qualification (AGR) program's second irradiation test, AGR-2. The data in this summary document is being provided to Robert P Wichner in support of a Nuclear Regulatory Commission (NRC) Methods for Estimation of Leakages and Consequences Of Release (MELCOR) code development effort.

The AGR-2 compacts described in this report contained two types of fuel kernels. Uranium oxide $\left(\mathrm{UO}_{2}\right)$ fuel kernels with a nominal diameter of $500 \mu \mathrm{m}$ and mixed uranium oxide/uranium carbide (UCO) fuel kernels with a nominal diameter of $425 \mu \mathrm{m}$. The fuel kernels were produced by Babcock and Wilcox (B\&W) in Lynchburg Virginia. The UCO kernels were identified as composite G73I-14-69307. The $\mathrm{UO}_{2}$ kernels were identified as composite G73AA-10-69308.

The fuel kernels were coated with a tristructural-isotropic (TRISO) coating in a $150 \mathrm{~mm}$ diameter fluidized bed chemical vapor deposition (CVD) coating furnace at B\&W. The TRISO-coated particles consisted of a spherical kernel coated with an approximately $50 \%$ dense carbon buffer layer (100 $\mu \mathrm{m}$ nominal thickness), followed by a dense inner pyrocarbon layer ( $40 \mu \mathrm{m}$ nominal thickness), followed by a SiC layer $(35 \mu \mathrm{m}$ nominal thickness), followed by another dense outer pyrocarbon layer ( $40 \mu \mathrm{m}$ nominal thickness).

The TRISO-coated particles were shipped to Oak Ridge National Laboratory (ORNL) to be fabricated into the final fuel form. Particles were overcoated in a resinated graphite powder and then these overcoated particles were pressed into cylindrical compacts. This document is a compilation of characterization data for four compact lots: AGR-2 UCO Variant fuel compact lot LEU06-OP1-Z, AGR-2 UCO Baseline fuel compact lot LEU07-OP1-Z, AGR-2 UCO Variant fuel compact lot LEU09-OP2-Z, and AGR-2 B\&W $\mathrm{UO}_{2}$ fuel compact lot LEU11-OP2-Z.

LEU06-0P1-Z was a compact lot fabricated using particle composite LEU06. LEU06 came from B\&W coated particle lot G73J-14-93074A, which was an upgraded batch of TRISO-coated $425 \mu \mathrm{m}$ diameter, $14 \%$ low enriched uranium oxide/uranium carbide kernels (LEUCO). LEU07-OP1-Z was a compact lot fabricated using particle composite LEU07. LEU07 came from B\&W coated particle lot G73J-14-93072A, which was an upgraded batch of TRISO-coated $425 \mu \mathrm{m}$ diameter, 14\% LEUCO. These two compact lots were fully characterized, but were not used in the AGR-2 irradiation test.

LEU09-0P2-Z was a compact lot fabricated using particle composite LEU09. LEU09 came from B\&W coated particle lot G73J-14-93073A, which was an upgraded batch of TRISO-coated $425 \mu \mathrm{m}$ diameter, 14\% LEUC0. LEU11-OP2-Z was a compact lot fabricated using particle composite LEU11. LEU11 came from B\&W coated particle lot G73H-1093085B, which was an upgraded batch of TRISO-coated $500 \mu \mathrm{m}$ nominal diameter, $9.6 \%$ low enriched uranium oxide kernels. These two compact lots were fully characterized and were used in the AGR-2 irradiation test. 


\section{Compact Fabrication}

After coating, particle batches were upgraded using sieves and shape separation tables. Material for compacting was then chute riffled from the final particle lots and shipped from $\mathrm{B} \& \mathrm{~W}$ to ORNL. Further upgrading using a roller-micrometer was performed at ORNL on particle batches LEU09 and LEU11 to remove a small number of bare kernels, undersized particles, and heavily faceted particles. The particles were then washed multiple times in methanol by submerging the particles and then decanting off the liquid. Washing of particles prior to overcoating was adapted in order to help reduce the amount of contamination on the particles that may have been acquired during processing or general handling. This procedure also reduces the amount of loose carbon dust on the surface of the particles.

The roller-micrometer technique uses rotating inclined cylinders with a diverging gap to sort particles according to their size. Using a vibrating vee-trough feeder, particles are fed in a single stream into the gap between the rollers. The rollers are angled downward away from the feed point and rotate with an upward and outward motion. Particles travel down the gradually widening gap until they reach a point equal to their width, at which point they drop through the gap into a series of collection bins. The roller-micrometer is a very accurate and reliable device for sorting coated particles by size. It also tends to sort coated particles by shape because the particles continuously re-orient as they travel down the inclined rollers, and faceted particles fall through a narrower gap than spherical particles of the same diameter. Figure 1 shows the roller-micrometer equipment.

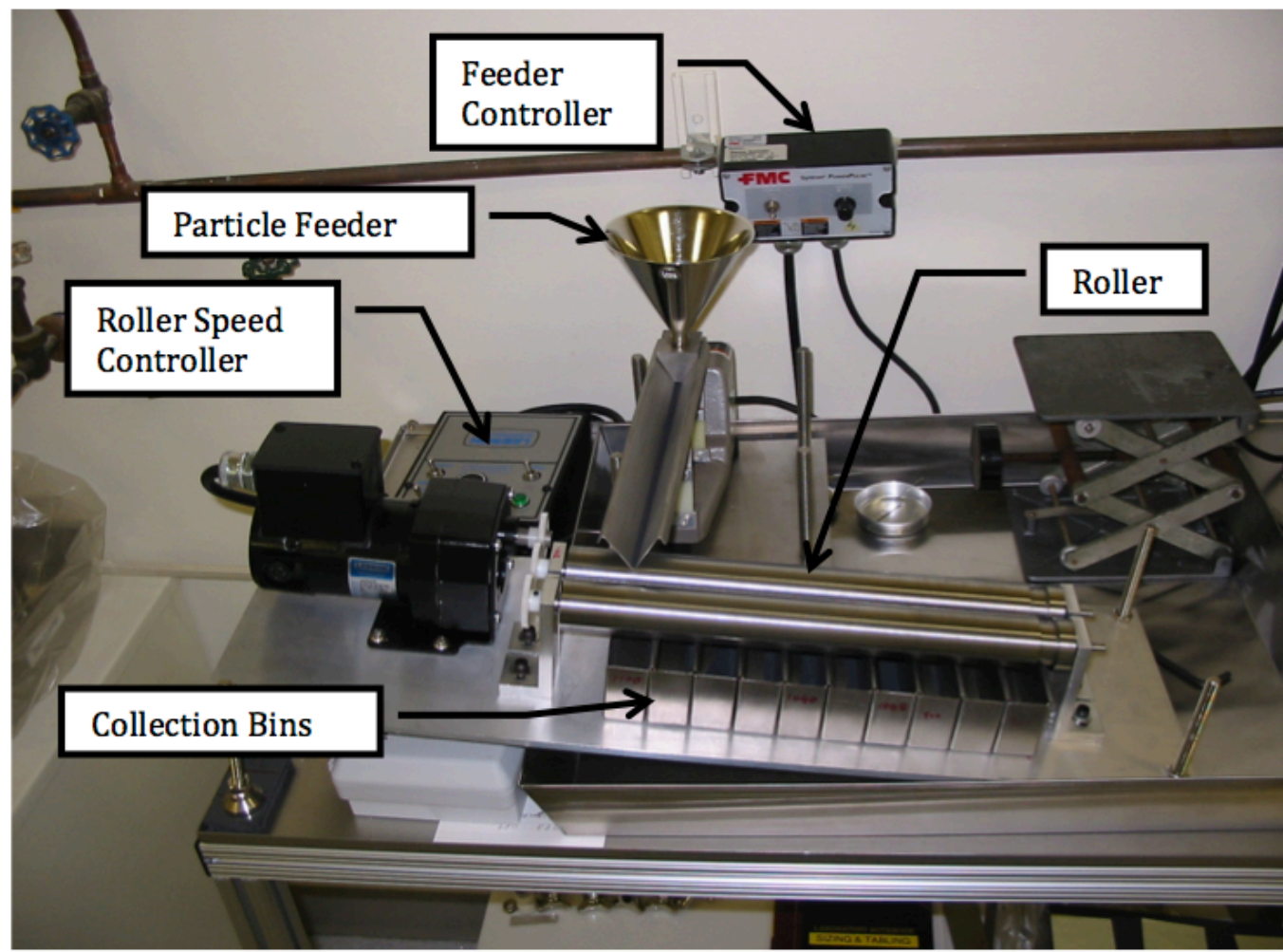

Figure 1. Photograph of roller-micrometer equipment showing the arrangement of the roller, particle feeder, and collection bins. 
After washing, particle samples were riffled out for characterization. Average particle weight, diameter, envelope volume, and uranium content were measured. At this time, 20 gram aliquots for use as overcoater charges were also riffled. Riffling at ORNL was done using a 10 position rotary riffler.

Particles were overcoated by rolling in a matrix precursor prepared from a resinated mix of natural and synthetic graphite. The AGR-2 Fuel Specification (SPC-923) puts maximum limits on the elemental impurities $\mathrm{Al}, \mathrm{Ca}, \mathrm{Ti}, \mathrm{V}, \mathrm{Cr}, \mathrm{Mn}, \mathrm{Fe}, \mathrm{Co}$, and $\mathrm{Ni}$. The natural graphite, synthetic graphite, and thermosetting resin used to make the matrix/overcoat material may contain these impurities. Therefore, the selection of graphite and resin used to make the matrix must have low concentrations of these impurities to ensure that compacts made using this matrix will be within specification. Part of the compacting development effort was selection and qualification of natural graphite, synthetic graphite, and resin. A graphite or resin was considered "qualified" if it could produce a compact that was within specification on impurities. The qualification process involved receiving natural graphite and synthetic graphite and testing them via glow discharge mass spectrometry (GDMS) in order to establish their initial impurity concentrations. The graphite and resin were then combined to produce matrix that was carbonized and heat treated in powder form. The impurity levels in the heat treated matrix were then measured by GDMS as well.

Table 1 shows the initial impurity levels for the natural graphite and synthetic graphite that were used to make all the AGR-2 compacts. Natural graphite (Asbury Graphite Mills RD13371), synthetic graphite (SGL Carbon KRB2000), and a thermosetting resin were combined in a weight ratio of 64:16:20 to make the matrix. A sample of one of the matrix batches (RDKrS-050809) used to make LEU09-0P2-Z is also shown in Table 1. The matrix was carbonized and heat treated in powder form prior to being tested for impurities by GDMS. Notice that the heat treatment processes significantly reduced impurity levels in the matrix for several elements. The vanadium impurity in the heat treated matrix is higher than in the graphite and is most likely being picked up during heat treatment in the graphite furnace. Low levels of vanadium (15-20 $\mu \mathrm{g} / \mathrm{compact})$ have been observed in all the AGR-2 compacts. Uranium in all of the matrix components was less than the detection limit.

Table 1. Matrix constituents that were used in AGR-2 LEU09-OP2-Z compacts

\begin{tabular}{|c||c|c|c|}
\hline \multicolumn{1}{|c||}{\multirow{2}{*}{ Element }} & \multicolumn{3}{|c|}{ Impurity concentration (ppm) } \\
\cline { 2 - 4 } & $\begin{array}{c}\text { Natural graphite- } \\
\text { RD13371 }\end{array}$ & $\begin{array}{c}\text { Synthetic graphite- } \\
\text { KRB2000 }\end{array}$ & $\begin{array}{c}\text { Heat-treated matrix- } \\
\text { RDKrS-050809 }\end{array}$ \\
\hline \hline $\mathrm{Al}$ & 36 & 0.35 & 1.2 \\
\hline $\mathrm{Ca}$ & 9.4 & 0.7 & 0.51 \\
\hline $\mathrm{Ti}$ & 0.43 & 0.06 & 0.92 \\
\hline $\mathrm{V}$ & 0.6 & 0.02 & 8.8 \\
\hline $\mathrm{Cr}$ & 4.5 & $<0.5$ & $<0.5$ \\
\hline $\mathrm{Mn}$ & 0.54 & $<0.05$ & 0.05 \\
\hline $\mathrm{Fe}$ & 34 & 1.4 & $<0.05$ \\
\hline $\mathrm{Co}$ & $<0.05$ & 0.25 & $<0.1$ \\
\hline $\mathrm{Ni}$ & 0.37 & 1.2 & $<0.05$ \\
\hline $\mathrm{U}$ & $<0.05$ & $<0.05$ & \\
\hline
\end{tabular}


Overcoated particles were sorted for size by sieving and, in some cases, sorting by roller-micrometer. This process helped to control the average volume of overcoat on the particles (the amount of overcoat effects the final matrix density). The average weight per overcoated particle was determined and was used in conjunction with the average uranium per particle to calculate a compacting charge weight that would produce compacts meeting the specifications on dimension, matrix density, and uranium loading. A record of the weight of each compact charge can be found on data report form DRF-24D in Appendix A.

Note that all the uranium per particle measurements on the LEU06, LEU07 and LEU09 material were consistent to within the sampling error and measurement uncertainty, as expected given that the same kernel batch was used for all three coating runs. Table 2 shows the measured average uranium content per particle obtained directly from the uranium analyses of the individual particles compared to that calculated from the uranium analyses of the compacts divided by the average number of particles per compact.

Table 2. Average and standard deviation ( \pm value) for uranium per particle based on particle and compact analyses of LEU06, LEU07 and LEU09

\begin{tabular}{|l|c|c|c|}
\hline & LEU06 & LEU07 & LEU09 \\
\hline \hline $\begin{array}{l}\text { grams U/particle } \\
\text { based on particle analysis }\end{array}$ & $3.953 \pm 0.010 \cdot 10^{-4}$ & $3.930 \pm 0.007 \cdot 10^{-4}$ & $3.964 \pm 0.009 \cdot 10^{-4}$ \\
\hline $\begin{array}{l}\text { grams U/particle } \\
\text { based on compact analysis }\end{array}$ & $3.942 \pm 0.006 \cdot 10^{-4}$ & $3.938 \pm 0.016 \cdot 10^{-4}$ & $3.958 \pm 0.009 \cdot 10^{-4}$ \\
\hline
\end{tabular}

A significant change was made to the compacting equipment between the LEU06 and LEU07 campaigns and the LEU09 and LEU11 campaigns. A Carver manual hydraulic press was replaced with a Promess servo-electric press. The Promess press provided pressing rate and piston displacement control to three decimal place precision. This enabled the pressing rate and compact length to be precisely set and repeated for each individual compacting charge. The force for each compact was also recorded more accurately, to $\pm 10 N$. Figure 2 and Figure 3 show the distribution in compact length for LEU06-0P1-Z and LEU09-0P2-Z. The effect of the introduction of the Promess press can clearly be seen. The AGR-2 specified compact length limits of $\geq 25.02 \mathrm{~mm}$ and $\leq 25.40 \mathrm{~mm}$ are shown in the figures as vertical lines. 


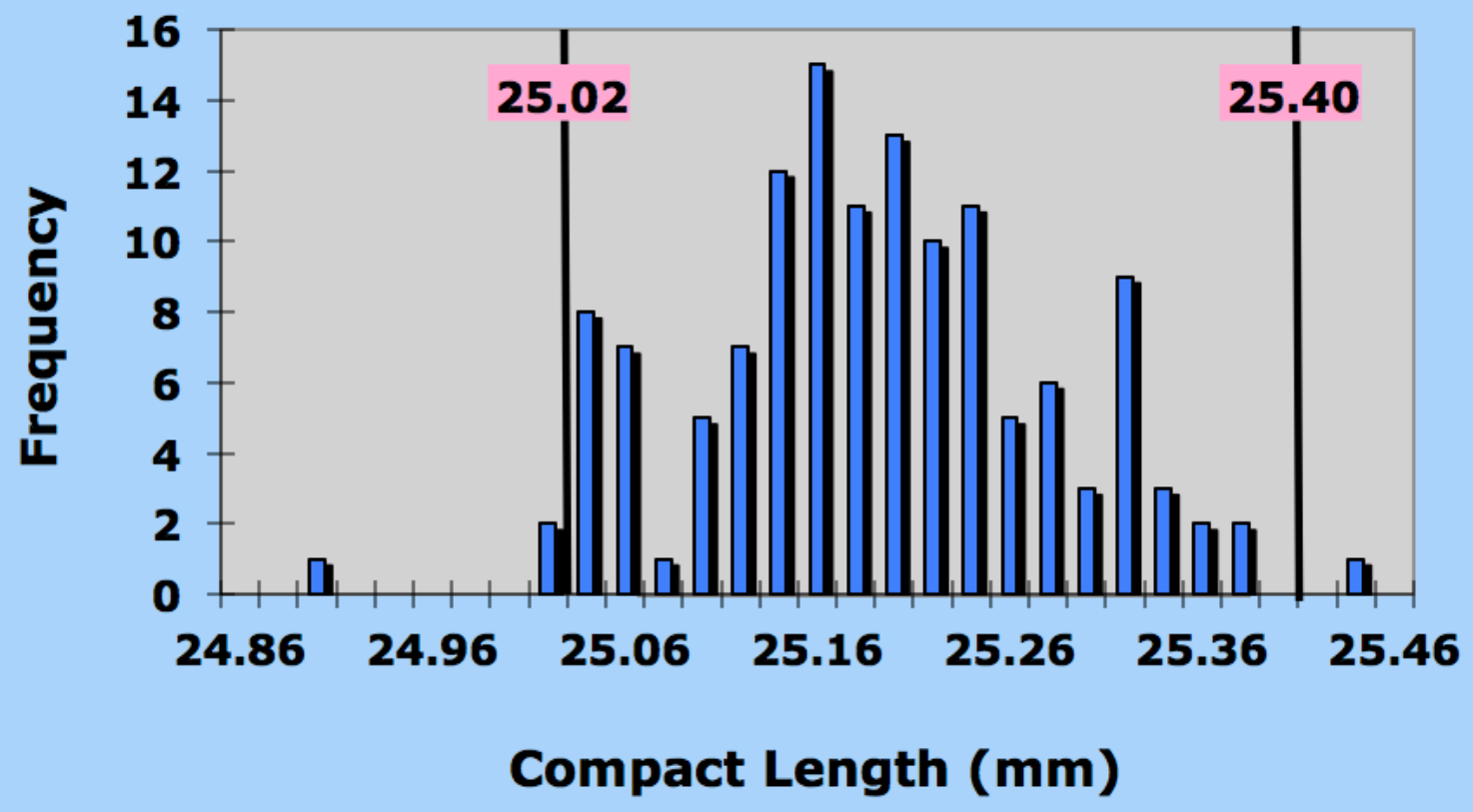

Figure 2. LEU06-OP1-Z compact length distribution.

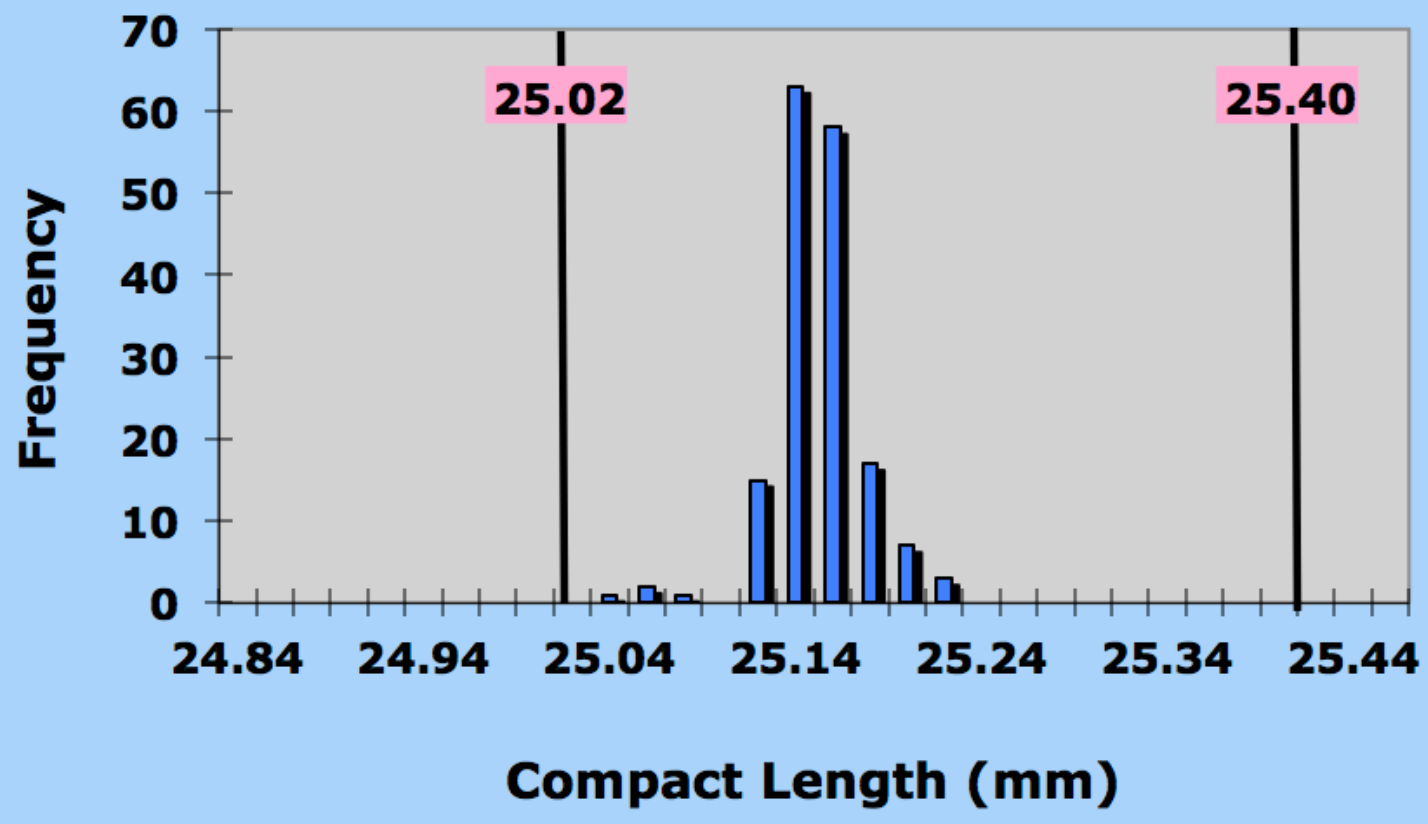

Figure 3. LEU09-OP2-Z compact length distribution. 
The compacting charges were formed into green compacts using a heated, double acting die. The die was heated between $65^{\circ} \mathrm{C}$ and $75^{\circ} \mathrm{C}$ (except for LEU06, which was pressed at $95^{\circ} \mathrm{C}$ ). Approximately $0.10 \mathrm{~g}$ of matrix was added to the top and bottom of the compact in order to create matrix "end caps." The end caps were formed with the compact by first pouring a matrix charge into the heated die, followed by the overcoated particles, and then a second charge of matrix. This forming method created a thin $(<0.25 \mathrm{~mm}$ thick) fuel free zone on the ends of the compact, called end caps. These fuel free zones can be seen in the $\mathrm{x}$ ray images in Figure 4. Molding pressure was less than $60 \mathrm{MPa}$. In total, 180-220 green compacts were fabricated. The compacts retained the designation of the riffled charges (e.g., LEU09-OP2-G001 through G185). The green compacts were carbonized and heat treated according to the following procedures.

$\begin{array}{ll}\text { Carbonization parameters: } & <350^{\circ} \mathrm{C} / \mathrm{hr} \text { in He atmosphere } \\ & \text { Hold at } 950 \pm 50^{\circ} \mathrm{C} \text { for } 1.0 \pm 0.4 \mathrm{hr} \\ & \text { Furnace cool }\end{array}$

Heat treatment parameters: $\quad \sim 20^{\circ} \mathrm{C} / \mathrm{min}$ in vacuum $(<1.3 \mathrm{~Pa})$

Hold at $1650-1850^{\circ} \mathrm{C}$ for $60 \pm 10 \mathrm{~min}$

Furnace cool at $\sim 20^{\circ} \mathrm{C} / \mathrm{min}$ to below $700^{\circ} \mathrm{C}$

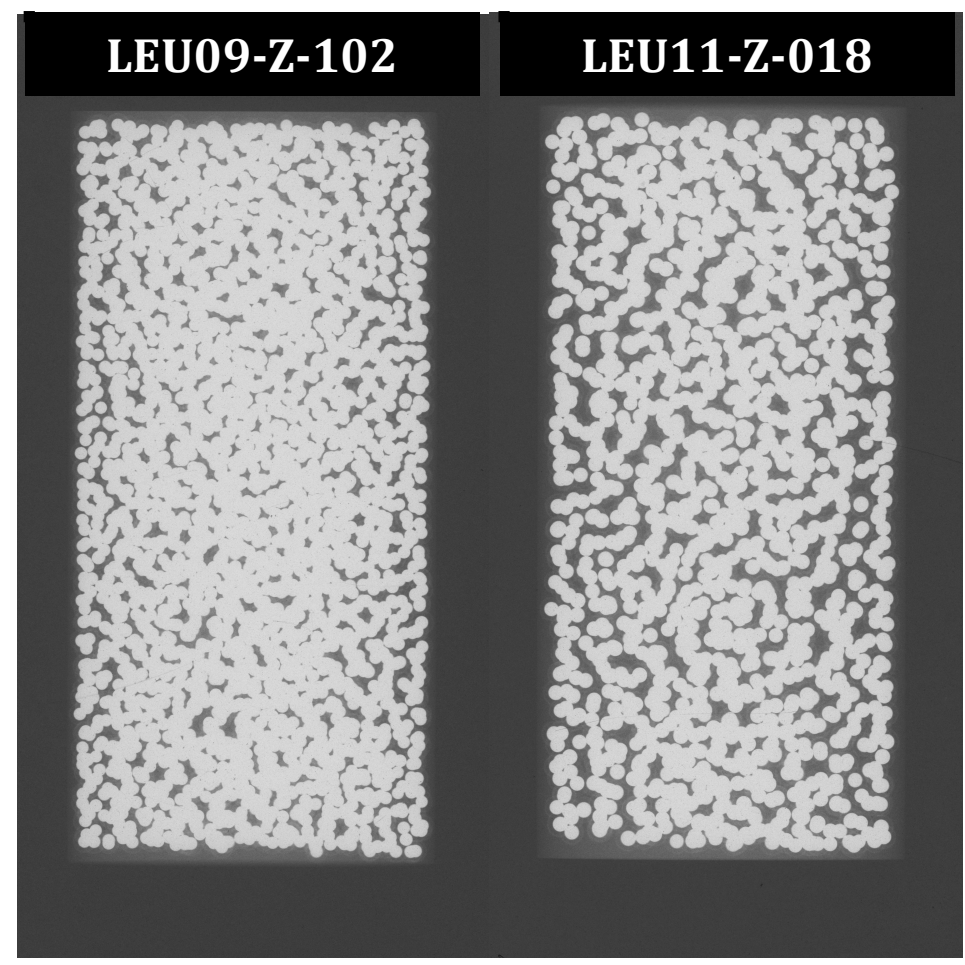

Figure 4. X-ray radiographs of compacts from LEU09-OP2-Z and LEU11-OP2-Z. 
After compacting, an appropriate number of compacts were selected for use. Compacts with obvious processing defects, chips, or undesirable dimensions were sorted out and not included in the compacts selected for the final fuel compact lot. This down-select was part of the compacting process and was performed prior to random selection of compacts for acceptance testing. The selected compacts were randomized and relabeled, changing the Gnumber designation to a Z-number designation. A record of the original G-number for each Z-numbered compact can be found on data report form DRF-24C in Appendix A. After relabeling, the compacts were characterized for product acceptance. This included measurement of compact length, diameter, mass, matrix density, uranium content, and impurity content. In addition, analyses were performed to determine defect fractions for exposed uranium, defective $\mathrm{SiC}$, uranium dispersion due to defective inner pyrocarbon (IPyC), and defective outer pyrocarbon (OPyC). 


\section{Summary of Particle and Compact Properties}

The appendices to this report provide copies of some of the data report forms (DRF) generated during characterization of the particles and compacts. These forms contain the detailed data generated by the various inspections performed. Appendix A contains information on the compact length, diameter, mass measured for each individual compact and the calculated average packing fraction and average matrix density. Appendix B contains information on the particle diameter measured on individual particles using a shadowscope technique and average particle envelope volume measured on several thousand particles by mercury displacement. Appendix C contains information on the average uranium loading per particle and per compact.

Table 3 gives mean values and standard deviations for key variable properties of the compact lots and associated particle batches. Data report forms associated with the summary data in Table 3 are attached in the appendices to this report.

Table 3. Quick reference table for key variable properties

\begin{tabular}{|c|c|c|c|c|}
\hline Property & LEU06 & LEU07 & LEU09 & LEU11 \\
\hline Mean particle diameter $(\mu \mathrm{m})$ & $\begin{array}{c}874.7 \\
(22)\end{array}$ & $\begin{array}{c}861.8 \\
(24)\end{array}$ & $\begin{array}{c}873.2 \\
(23)\end{array}$ & $\begin{array}{c}953.0 \\
(28)\end{array}$ \\
\hline Mean particle volume (cc) & $3.46 \mathrm{E}-4$ & $3.29 \mathrm{E}-4$ & $3.43 \mathrm{E}-4$ & $4.45 \mathrm{E}-4$ \\
\hline Mean particle weight (g) & $\begin{array}{c}1.036 \mathrm{E}-3 \\
(2 \mathrm{E}-6)\end{array}$ & $\begin{array}{c}1.004 \mathrm{E}-4 \\
(5 \mathrm{E}-6)\end{array}$ & $\begin{array}{c}1.032 \mathrm{E}-3 \\
(3 \mathrm{E}-6)\end{array}$ & $\begin{array}{c}1.462 \mathrm{E}-3 \\
(5 \mathrm{E}-6)\end{array}$ \\
\hline Mean uranium per particle (g $\mathrm{U}$ ) & $\begin{array}{c}3.95 \mathrm{E}-4 \\
(2 \mathrm{E}-6)\end{array}$ & $\begin{array}{c}3.93 \mathrm{E}-4 \\
(1 \mathrm{E}-6)\end{array}$ & $\begin{array}{c}3.96 \mathrm{E}-4 \\
(1 \mathrm{E}-6)\end{array}$ & $\begin{array}{c}6.39 \mathrm{E}-4 \\
(1 \mathrm{E}-6)\end{array}$ \\
\hline Average numbers of particles/compact & 3186 & 3177 & 3176 & 1543 \\
\hline Mean uranium per compact (g U) & $\begin{array}{c}1.256 \\
(0.002)\end{array}$ & $\begin{array}{c}1.251 \\
(0.005)\end{array}$ & $\begin{array}{c}1.257 \\
(0.003)\end{array}$ & $\begin{array}{c}0.993 \\
(0.006)\end{array}$ \\
\hline $\begin{array}{l}\% \text { packing fraction (particle volume / compact } \\
\text { volume) }\end{array}$ & $\begin{array}{l}37.08 \\
(0.15)\end{array}$ & $\begin{array}{l}35.09 \\
(0.12)\end{array}$ & $\begin{array}{l}36.81 \\
(0.05)\end{array}$ & $\begin{array}{l}23.46 \\
(0.04)\end{array}$ \\
\hline Mean compact diameter $(\mathrm{mm})$ & $\begin{array}{c}12.29 \\
(0.011)\end{array}$ & $\begin{array}{c}12.32 \\
(0.007)\end{array}$ & $\begin{array}{c}12.29 \\
(0.004)\end{array}$ & $\begin{array}{c}12.27 \\
(0.008)\end{array}$ \\
\hline Mean compact length (mm) & $\begin{array}{l}25.18 \\
(0.09)\end{array}$ & $\begin{array}{l}25.19 \\
(0.07)\end{array}$ & $\begin{array}{l}25.14 \\
(0.02)\end{array}$ & $\begin{array}{l}25.13 \\
(0.02)\end{array}$ \\
\hline Mean compact mass (g) & $\begin{array}{c}6.254 \\
(0.011)\end{array}$ & $\begin{array}{c}6.346 \\
(0.008)\end{array}$ & $\begin{array}{c}6.295 \\
(0.012)\end{array}$ & $\begin{array}{c}6.100 \\
(0.017)\end{array}$ \\
\hline Mean compact matrix density $\left(\mathrm{g} / \mathrm{cm}^{3}\right)$ & $\begin{array}{c}1.56 \\
(0.012) \\
\end{array}$ & $\begin{array}{c}1.61 \\
(0.008)\end{array}$ & $\begin{array}{c}1.59 \\
(0.005)\end{array}$ & $\begin{array}{c}1.68 \\
(0.008)\end{array}$ \\
\hline
\end{tabular}

Values in parentheses are standard deviations of the mean.

Table 4 summarizes impurity data for the AGR-2 fuel compacts. This data was obtained by leach-burn-leach (LBL) followed by inductively coupled plasma mass spectrometry analysis of the nitric acid leach solutions. The table shows the possible range for the mean value of the measured impurities, where the upper limit is the as-reported mean and the lower limit is the possible minimum value calculated by accounting for the fact that the analytical mass spectrometer measurements reported as threshold values could have been 
as low as zero. This range reflects the uncertainty in the measured impurity values due to the mass spectrometry measurement thresholds.

Table 4. Mean impurity levels in $\mu \mathrm{g} /$ compact for AGR-2 fuel compacts measured by deconsolidation leach-burn-leach technique

\begin{tabular}{|l|c|c|c|c|}
\hline Impurity & LEU06 & LEU07 & LEU09 & LEU11 \\
\hline \hline Iron & $1.43-4.86$ & $0.70-4.78$ & $0.51-4.04$ & $0.13-2.75$ \\
\hline Chromium & $0.30-0.64$ & $0.37-0.59$ & $0.46-0.61$ & $0.34-0.48$ \\
\hline Manganese & $0.31-0.84$ & $0.00-0.20$ & $0.000-0.136$ & $0.000-0.133$ \\
\hline Cobalt & $0.00-0.26$ & $0.00-0.16$ & $0.000-1.115$ & $0.000-0.113$ \\
\hline Nickel & $0.00-1.28$ & $0.02-0.84$ & $0.38-0.96$ & $0.02-0.59$ \\
\hline Calcium & $39.23-40.78$ & $30.35-36.05$ & $36.20-39.34$ & $34.29-35.16$ \\
\hline Aluminum & $31.37-31.48$ & $30.01-30.08$ & $29.60-29.60$ & 42.69 \\
\hline Titanium & $7.77-9.12$ & $2.17-3.05$ & $2.20-2.81$ & $2.76-3.31$ \\
\hline Vanadium & $20.06-20.37$ & $18.01-18.23$ & $16.94-17.09$ & $15.27-15.41$ \\
\hline
\end{tabular}

Table 5 is a summary of the measured defect fractions for AGR-2 fuel compacts. Listed in the table are the actual numbers of defects observed for the numbers of particles analyzed. In parentheses are the binomial distribution calculated upper limits of the 95\% confidence interval on the various defect fractions. In other words, these values are the lowest tolerance limits for which the compact lot would be deemed acceptable at 95\% confidence, based on the particular sample that was measured. In many cases zero defects were observed. The defect fractions listed in the table for these cases are limited by the number of particles measured. The actual defect fraction could be much lower.

Table 5. Quick reference table for key attribute properties

\begin{tabular}{|c|c|c|c|c|}
\hline Defect Property & LEU06 & LEU07 & LEU09 & LEU11 \\
\hline \multirow{2}{*}{ Exposed kernel fraction } & $\begin{array}{c}10 / 127448 \\
(\leq 1.4 \mathrm{E}-4)\end{array}$ & $\begin{array}{c}15 / 317690 \\
(\leq 7.3 \mathrm{E}-5)\end{array}$ & $\begin{array}{c}3 / 317625 \\
(\leq 2.5 \mathrm{E}-5)\end{array}$ & $\begin{array}{c}3 / 246840 \\
(\leq 3.2 \mathrm{E}-5)\end{array}$ \\
\hline \multirow{2}{*}{ Defective SiC coating fraction } & $0 / 127448$ & $0 / 190614$ & $0 / 254100$ & $0 / 123420$ \\
& $(\leq 2.4 \mathrm{E}-5)$ & $(\leq 1.6 \mathrm{E}-5)$ & $(\leq 1.2 \mathrm{E}-5)$ & $(\leq 2.5 \mathrm{E}-5)$ \\
\hline \multirow{2}{*}{ Defective IPyC coating fraction } & $0 / 63724$ & $443 / 63538$ & $0 / 63525$ & $1 / 61710$ \\
& $(\leq 4.8 \mathrm{E}-5)$ & $(\leq 7.6 \mathrm{E}-3)$ & $(\leq 4.8 \mathrm{E}-5)$ & $(\leq 7.7 \mathrm{E}-5)$ \\
\hline \multirow{2}{*}{ Defective OPyC coating fraction } & $0 / 3186$ & $0 / 3177$ & $0 / 3176$ & $0 / 1543$ \\
& $(\leq 9.4 \mathrm{E}-4)$ & $(\leq 9.5 \mathrm{E}-4)$ & $(\leq 9.5 \mathrm{E}-4)$ & $(\leq 2.0 \mathrm{E}-3)$ \\
\hline
\end{tabular}

Values in parentheses are 95\% confidence limits.

Exposed kernel defects are those kernels that are not protected by any coating layer, which can be due to fracture of the TRISO coating during particle handling operations or coating damage during compacting. The fraction of exposed kernels is determined from the dissolved uranium in the pre-burn leach solutions during deconsolidation-LBL analysis. Particles with $\mathrm{SiC}$ coating defects are particles whose kernels become exposed only after removal of exposed carbon during the burn step of the LBL analysis. This can indicate porous or cracked $\mathrm{SiC}$ layers that may not retain fission products during reactor operation. The fraction of particles with $\mathrm{SiC}$ coating defects is determined from the dissolved uranium in the post-burn leach solutions. Particles with defective IPyC coatings are particles that 
allow unacceptable chlorine intrusion through the IPyC layer during SiC deposition. When particles are at an elevated temperature during coating and compacting, chlorine in the buffer layer can react with uranium in the kernel, causing it to migrate out of the kernel. Particles with defective IPyC coatings are detected by using x-ray imaging to look for excessive uranium dispersion in the buffer layer of particles deconsolidated from compacts. Particles with $\mathrm{OPyC}$ coating defects are particles that exhibit missing or damaged outer pyrocarbon, a condition that can occur during particle handling or compacting. The fraction of particles with defective $\mathrm{OPyC}$ is determined by visual inspection of particles deconsolidated from compacts.

The AGR-2 Fuel Specification (INL SPC-923) included a requirement for a uranium contamination fraction (grams exposed $U$ per grams $U$ in compact) of $\leq 2 \mathrm{E}-5$ at $95 \%$ confidence. Uranium contamination is defined as uranium that is not encapsulated by a fission gas retentive coating layer. It is determined from analysis of the first series of acid leach solutions obtained by LBL (before the burn) and includes any exposed kernels, uranium in the compact's graphite matrix, and uranium near the surface of the TRISO particles. For AGR-2 compacts, the total uranium contamination fraction was dominated by the exposed kernel fraction described above. The relatively high fractions of particles with exposed kernels reported in Table 5 was determined to most likely have occurred at B\&W during removal of the particles from the coating furnace via a suction transfer system. Compact lots LEU06-0P1-Z and LEU07-0P1-Z were rejected primarily because of these defects (LEU07 also had an unacceptable fraction of particles with defective IPyC). The uranium contamination fractions for LEU09-0P2-Z and LEU11-0P2-Z were also above the specified limit at 95\% confidence, but these compacts were dispositioned as acceptable for use in the irradiation. This disposition was partially justified by the fact that the actual measured defect fractions were less than 2E-5 and analysis of a larger sample size may have eventually provided data that would satisfy the specification at $95 \%$ confidence.

Improvements in the particle handling systems have been made at $B \& W$ as a result of the lessons learned from the AGR-2 fuel fabrication campaign. Future exposed kernel fractions are expected to be significantly reduced from the levels reported in Table 5. In the LBL analysis, compacts were inspected in sets of five compacts each. It is useful to consider the results from the sample sets that did not contain compacts with exposed kernel defects. The total amount of uranium not contained by $\mathrm{SiC}$ can be determined by analyzing all the acid leach solutions obtained by LBL (both before and after the burn). Table 6 summarizes this analysis for only those fuel compact sets with no exposed kernels or defective SiC. This provides a measure of the uranium contamination in the outer pyrocarbon and graphite matrix.

Table 6. Uranium not contained by $\mathrm{SiC}$ in compacts with intact $\mathrm{SiC}$

\begin{tabular}{|c|c|c|c|c|}
\hline Defect Property & LEU06 & LEU07 & LEU09 & LEU11 \\
\hline $\begin{array}{c}\text { Number of compacts analyzed with } \\
\text { no exposed kernels or defective SiC }\end{array}$ & 10 & 20 & 65 & 75 \\
\hline $\begin{array}{c}\text { Total uranium measured by LBL } \\
\text { (gU) }\end{array}$ & $2.82 \mathrm{E}-5$ & $1.04 \mathrm{E}-4$ & $3.18 \mathrm{E}-4$ & $7.04 \mathrm{E}-5$ \\
\hline $\begin{array}{c}\text { Fraction of uranium outside SiC } \\
\text { (gU/gU in compact) }\end{array}$ & $2.24 \mathrm{E}-6$ & $4.14 \mathrm{E}-6$ & $3.89 \mathrm{E}-6$ & $9.45 \mathrm{E}-7$ \\
\hline
\end{tabular}




\section{Appendix A : Compact dimensions, mass, matrix density, and packing fraction}

The diameter of a nominally cylindrical compact is inspected by measuring two orthogonal thicknesses near the top, middle and bottom using a digital caliper. Because these six chord measurements do not determine maximum diameter at all points or curvature of the compact, the conformance of the compact to fit within a cylinder of specified maximum diameter is checked by passing through a $12.460(+0.000 /-0.001) \mathrm{mm}$ inner diameter and 20-mm-thick ring gauge. The length of a compact is determined by measuring the distance between the ends of the compact using a vertical height gauge. The two contact faces of the height gauge are parallel and larger in diameter than the ends of the compact.

Average matrix density is calculated from

$$
\frac{\text { matrix } \text { weight }}{\text { matrix volume }}=\frac{\text { compact weight }- \text { particle weight }}{\text { compact volume }- \text { particle volume }} \text {. }
$$

Compact weight is measured directly on each compact. Compact volume is calculated for each compact from the measured length and average of the measured diameters, assuming a cylindrical geometry. Total particle weight is estimated from the average weight per TRISO particle multiplied by the number of particles in the compact. The number of particles in the compact is estimated from the total weight of the overcoated particles used in the compact charge divided by the average weight per overcoated particle.

particle weight $=$ average weight per TRISO particle $\times$ approximate number of particles

$$
\text { approximate number of particles }=\text { Round to Integer }\left(\frac{\text { overcoated particle weight }}{\text { average weight per overcoated particle }}\right)
$$

The particle volume is estimated from the average TRISO particle volume multiplied by the number of particles in the compact, which is estimated as for particle weight.

particle volume $=$ average volume per TRISO particle $\times$ approximate number of particles

Average packing fraction is determined as the total particle volume divided by the compact volume.

This appendix contains information on the compact length, diameter, mass for each individual compact in DRF-24A and the calculated packing fraction and matrix density in DRF-24B. 
Data Report Form DRF-24A: compact Dlameter and Length

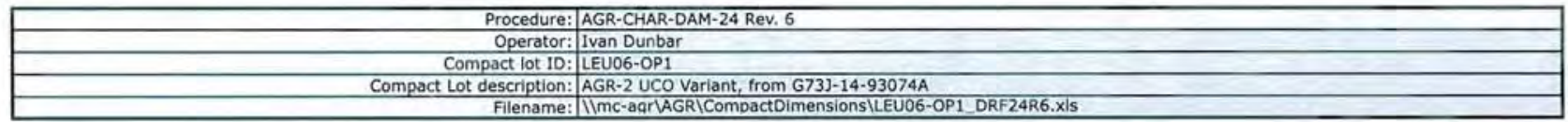

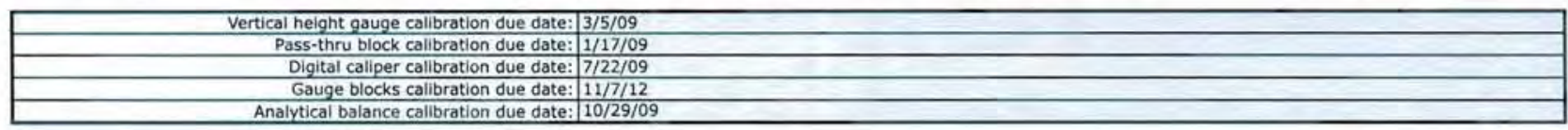

\begin{tabular}{|c|l|}
\hline Acceptance criteria for compact length: & 225.02 and $\leq 25.40 \mathrm{~mm}$ \\
\hline Acceptance criteria for compact diameter: & 212.22 and $\leq 12.46 \mathrm{~mm}$ (and pass through $12.46 \mathrm{~mm}$ ring gauge) \\
\hline Acceptance criteria for compact mass: & For information only \\
\hline
\end{tabular}

\begin{tabular}{|c|c|c|c|c|c|c|c|c|c|c|}
\hline \multirow{2}{*}{$\begin{array}{l}\text { Compact } \\
\text { ID Number }\end{array}$} & \multirow{2}{*}{$\begin{array}{l}\text { Length } \\
(\mathrm{mm})\end{array}$} & \multicolumn{6}{|c|}{ Diameter $(\mathrm{mm})$} & \multirow{2}{*}{$\begin{array}{l}\text { Pass Thru? } \\
\text { (Yor } \mathrm{N})\end{array}$} & \multirow{2}{*}{$\begin{array}{l}\text { Compact weight } \\
\text { (a) }\end{array}$} & \multirow{2}{*}{$\begin{array}{c}\text { Accept? } \\
\text { (pass or fall) }\end{array}$} \\
\hline & & Top 1 & Top 2 & Middle 1 & Middle 2 & Bottom 1 & Bottom 2 & & & \\
\hline & & 12.29 & 12.27 & 12.29 & 12.29 & 12.28 & 12.28 & & & \\
\hline 2002 & 25.025 & 12.28 & 12.28 & 12.29 & 12.29 & 12.28 & 12.29 & $\mathrm{Y}$ & 6.2585 & pass \\
\hline 2003 & 25.192 & 12.29 & 12.28 & 12.30 & 12.30 & 12.30 & 12.30 & $\mathrm{Y}$ & 6.2521 & pass \\
\hline 2004 & 25.133 & 12.28 & 12.27 & 12.29 & 12.29 & 12.28 & 12.28 & $\bar{Y}$ & 6.2508 & pass \\
\hline $\mathrm{ZO05}$ & 25.003 & 12.29 & 12.28 & 12.29 & 12.30 & 12.28 & 12.28 & $\bar{Y}$ & 6.2698 & fall \\
\hline 2006 & 25.099 & 12.27 & 12.27 & 12.28 & 12.28 & 12.28 & 12.27 & $Y$ & 6.2659 & pass \\
\hline 2007 & 25.265 & 12.30 & 12.30 & 12.30 & 12.29 & 12.31 & 12.30 & $\mathrm{Y}$ & 6.2593 & pass \\
\hline 2008 & 25.242 & 12.28 & 12.28 & 12.28 & 12.28 & 12.28 & 12.28 & $\bar{Y}$ & 6.2668 & pass \\
\hline 2009 & 25.199 & 12.29 & 12.28 & 12.29 & 12.30 & 12.27 & 12.28 & Y & 6.2563 & pass \\
\hline 2010 & 25.031 & 12.28 & 12.29 & 12.29 & 12.29 & 12.27 & 12.27 & $\bar{Y}$ & 6.2610 & pass \\
\hline 2011 & 25.117 & 12.28 & 12.27 & 12.30 & 12.30 & 12.28 & 12.28 & $\mathrm{Y}$ & 6.2700 & pass \\
\hline 2012 & 25.318 & 12.29 & 12.30 & 12.30 & 12.30 & 12.28 & 12.28 & $Y$ & 6.2599 & pass \\
\hline 2013 & 25.173 & 12.28 & 12.29 & 12.29 & 12.30 & 12.30 & 12.28 & $Y$ & 6.2544 & pass \\
\hline 2014 & 25.235 & 12.29 & 12.29 & 12.30 & 12.30 & 12.29 & 12.29 & $\bar{Y}$ & 6.2670 & pass \\
\hline 2015 & 25.056 & 12.29 & 12.29 & 12.29 & 12.29 & 12.29 & 12.29 & $Y$ & 6.2614 & pass \\
\hline 2016 & 25.098 & 12.27 & 12.28 & 12.28 & 12.28 & 12.28 & 12.28 & Y & 6.2468 & pass \\
\hline 2017 & 25.095 & 12.28 & 12.27 & 12.29 & 12.29 & 12.27 & 12.28 & $r$ & 6.2747 & pass \\
\hline 2018 & 25.153 & 12.27 & 12.28 & 12.29 & 12.29 & 12.27 & 12.27 & $\bar{Y}$ & 6.2644 & pass \\
\hline 2019 & 25.173 & 12.27 & 12.28 & 12.28 & 12.28 & 12.28 & 12.28 & $r$ & 6.2449 & pass \\
\hline 2020 & 25.314 & 12.27 & 12.27 & 12.27 & 12.28 & 12.27 & 12.27 & $Y$ & 6.2618 & pass \\
\hline 2021 & 25.151 & 12.27 & 12.27 & 12.28 & 12.28 & 12.27 & 12.27 & $Y$ & 6.2629 & pass \\
\hline 2022 & 25.205 & 12.27 & 12.28 & 12.29 & 12.28 & 12.27 & 12.27 & $Y$ & 6.2646 & pass \\
\hline 2023 & 25.029 & 12.27 & 12.27 & 12.28 & 12.27 & 12.26 & 12.26 & 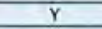 & 6.2530 & pass \\
\hline 2024 & 25.150 & 12.28 & 12.28 & 12.29 & 12.29 & 12.27 & 12.27 & $y$ & 6.2486 & pass \\
\hline 2025 & 25.149 & 12.27 & 12.28 & 12.29 & 12.29 & 12.28 & 12.28 & $Y$ & 6.2617 & pass \\
\hline 2026 & 25.225 & 12.28 & 12.27 & 12.29 & 12.28 & 12.27 & 12.28 & $\bar{Y}$ & 6.2462 & pass \\
\hline 2027 & 25.361 & 12.29 & 12.28 & 12.29 & 12.29 & 12.27 & 12.27 & $y$ & 6.2610 & pass \\
\hline 2028 & $\frac{25.301}{25.189}$ & 12.29 & 12.28 & $\frac{12.29}{12.29}$ & 12.29 & 12.28 & 12.27 & $\frac{1}{y}$ & $\frac{0.2010}{6.2592}$ & $\frac{\text { poss }}{\text { pass }}$ \\
\hline 2029 & 25.129 & 12.28 & 12.29 & 12.29 & 12.29 & 12.28 & 12.28 & $Y$ & 6.2610 & pass \\
\hline 2030 & 25.319 & 12.29 & 12.27 & 12.28 & 12.28 & 12.28 & 12.27 & $Y$ & 6.2406 & pass \\
\hline 2031 & 25.325 & 12.29 & 12.28 & 12.29 & 12.29 & 12.28 & 12.27 & $Y$ & 6.2613 & pass \\
\hline 2032 & 25.173 & 12.27 & 12.27 & 12.29 & 12.29 & 12.29 & 12.28 & $Y$ & 6.2553 & pass \\
\hline 2033 & 25.175 & 12.29 & 12.28 & 12.30 & 12.30 & 12.30 & 12.29 & $Y$ & 6.2416 & pass \\
\hline 2034 & 25.191 & 12.28 & 12.27 & 12.29 & 12.29 & 12.28 & 12.29 & $Y$ & 6.2526 & pass \\
\hline 2035 & 25.035 & 12.27 & 12.26 & 12.28 & 12.27 & 12.26 & 12.26 & $Y$ & 6.2667 & pass \\
\hline 2036 & 25.225 & 12.27 & 12.27 & 12.29 & 12.29 & 12.29 & 12.28 & Y & 6.2640 & pass \\
\hline 2037 & 25.148 & 12.28 & 12.27 & 12.29 & 12.29 & 12.29 & 12.28 & $Y$ & 6.2580 & pass \\
\hline 2038 & 25.151 & 12.26 & 12.26 & 12.27 & 12.27 & 12.26 & 12.27 & $\frac{1}{Y}$ & $\frac{0.2500}{6.2720}$ & $\frac{\text { pass }}{\text { pass }}$ \\
\hline Z039 & 25.057 & 12.27 & 12.27 & 12.28 & 12.27 & 12.27 & 12.27 & $\bar{Y}$ & 6.2644 & pass \\
\hline 2040 & 25.215 & 12.28 & 12.28 & 12.29 & 12.29 & 12.28 & 12.28 & $Y$ & 6.2623 & pass \\
\hline
\end{tabular}

Comments

Compact LEU06-0P1-Z005 was $0.017 \mathrm{~mm}$ below the lower limit on compact length; this compact was used for LBL analysis.

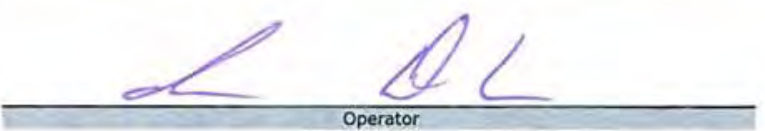

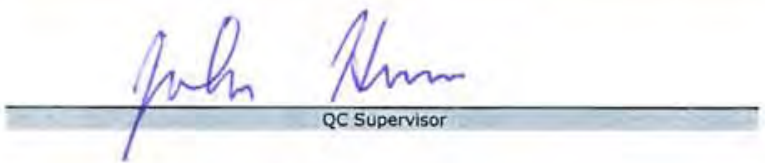

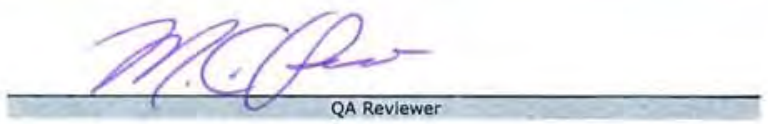

$$
2-27-09
$$

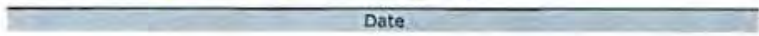

$7-24-09$
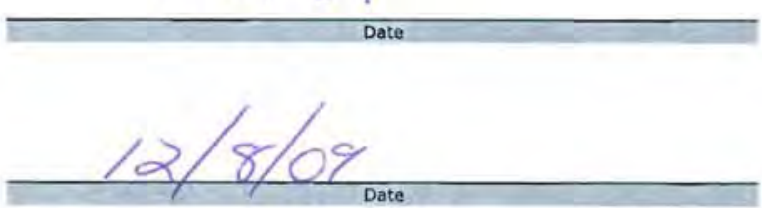
Data Report Form DRF-24A: Compact Diameter and Length

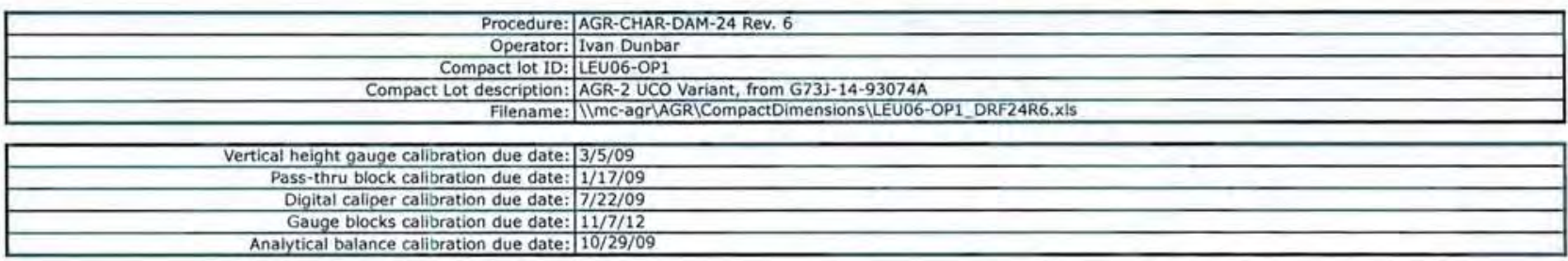

\begin{tabular}{|l|l|}
\hline Acceptance criteria for compact length: & $\geq 25.02$ and $\leq 25.40 \mathrm{~mm}$ \\
\hline Acceptance criteria for compact diameter: & $\geq 12.22$ and $\leq 12.46 \mathrm{~mm}$ (and pass through $12.46 \mathrm{~mm}$ ring gauge) \\
\hline Acceptance criteria for compact mass: & For information only \\
\hline
\end{tabular}

\begin{tabular}{|c|c|c|c|c|c|c|c|c|c|c|}
\hline \multirow{2}{*}{$\begin{array}{c}\text { Compact } \\
\text { to Number }\end{array}$} & \multirow{2}{*}{$\begin{array}{l}\begin{array}{l}\text { Length } \\
(\mathrm{mm})\end{array}\end{array}$} & \multicolumn{6}{|c|}{ Diameter $(\mathrm{mm})$} & \multirow{2}{*}{$\begin{array}{l}\text { Pass Thru? } \\
\text { (Y or } \mathrm{N}) \\
\end{array}$} & \multirow{2}{*}{$\begin{array}{l}\text { Compact weight } \\
\text { (a) }\end{array}$} & \multirow{2}{*}{$\begin{array}{c}\text { Accept? } \\
\text { (pass or fail) }\end{array}$} \\
\hline & & Top 1 & Top 2 & Middle 1 & Middle 2 & Bottom 1 & Bottom 2 & & & \\
\hline 2041 & & 12.28 & 12.27 & 12.29 & 12.29 & 12.28 & 12.28 & & & \\
\hline 2042 & 25.248 & 12.28 & 12.27 & 12.29 & 12.28 & 12.28 & 12.27 & $\bar{Y}$ & 6.2592 & pass \\
\hline 2043 & 25.059 & 12.28 & 12.27 & 12.29 & 12.29 & 12.27 & 12.28 & $\bar{Y}$ & 6.2391 & pass \\
\hline 2044 & 25.015 & 12.27 & 12.27 & 12.28 & 12.29 & 12.27 & 12.27 & $Y$ & 6.2207 & fail \\
\hline 2045 & 25.111 & 12.27 & 12.27 & 12.29 & 12.28 & 12.26 & 12.27 & $\frac{Y}{Y}$ & 6.2484 & pass \\
\hline 2046 & 25.236 & 12.26 & 12.27 & 12.28 & 12.27 & 12.27 & 12.27 & 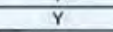 & 6.2633 & pass \\
\hline 2047 & 25.305 & 12.27 & 12.26 & 12.28 & 12.29 & 12.27 & 12.26 & $\mathrm{Y}$ & 6.2569 & pass \\
\hline 2048 & 25.180 & 12.28 & 12.28 & 12.29 & 12.30 & 12.28 & 12.29 & $Y$ & 6.2583 & pass \\
\hline 2049 & 25.340 & 12.28 & 12.28 & 12.29 & 12.29 & 12.28 & 12.28 & $Y$ & 6.2775 & pass. \\
\hline 2050 & 25.262 & 12.27 & 12.28 & 12.28 & 12.28 & 12.27 & 12.27 & $\bar{Y}$ & 6.2359 & pass \\
\hline 2051 & 25,133 & 12.26 & 12.26 & 12.29 & 12.28 & 12.26 & 12.27 & $Y$ & 6.2435 & pass \\
\hline 2052 & 25.205 & 12.27 & 12.26 & 12.28 & 12.28 & 12.27 & 12.27 & $Y$ & 6.2631 & pass \\
\hline 2053 & 25,123 & 12.27 & 12.26 & 12.28 & 12.29 & 12.28 & 12.27 & $Y$ & 6.2432 & pass \\
\hline 2054 & 25.270 & 12.28 & 12.29 & 12.30 & 12.29 & 12.28 & 12.29 & $Y$ & 6.2561 & pass \\
\hline 2055 & 25.175 & 12.28 & 12.28 & 12.29 & 12.28 & 12.28 & 12.27 & $Y$ & 6.2570 & pass \\
\hline 2056 & 25.182 & 12.28 & 12.27 & 12.29 & 12.29 & 12.27 & 12.28 & $\frac{1}{y}$ & 6.2478 & pass \\
\hline 2057 & 25.101 & 12.26 & 12.26 & 12.27 & 12.27 & 12.26 & 12.27 & $Y$ & 6.2667 & pass \\
\hline 2058 & 25.138 & 12.28 & 12.27 & 12.29 & 12.28 & 12.27 & 12.27 & $\bar{Y}$ & 6.2384 & pass \\
\hline 2059 & 25.292 & 12.28 & 12.27 & 12.28 & 12.28 & 12.28 & 12.28 & $Y$ & 6.2209 & pass \\
\hline 2060 & 25.157 & 12.27 & 12.28 & 12.28 & 12.28 & 12.27 & 12.28 & $Y$ & 6.2519 & pass \\
\hline 2061 & 25.148 & 12.28 & 12.29 & 12.28 & 12.28 & 12.27 & 12.28 & $Y$ & 6.2444 & pass \\
\hline 2062 & 25.264 & 12.28 & 12.28 & 12.28 & 12.28 & 12.27 & 12.27 & $Y$ & 6.2791 & pass \\
\hline 2063 & 25.214 & 12.27 & 12.27 & 12.29 & 12.28 & 12.27 & 12.27 & $\bar{Y}$ & 6.2543 & pass \\
\hline 2064 & 25.238 & 12.29 & 12.29 & 12.29 & 12.29 & 12.28 & 12.28 & $Y$ & 6.2576 & pass \\
\hline 2065 & 25.130 & 12.28 & 12.28 & 12.29 & 12.29 & 12.28 & 12.29 & $Y$ & 6.2621 & pass \\
\hline 2066 & 25.104 & 12.27 & 12.27 & 12.29 & 12.28 & 12.27 & 12.27 & $\bar{Y}$ & 6.2522 & pass \\
\hline 2067 & 25.118 & 12.27 & 12.28 & 12.29 & 12.29 & 12.28 & 12.28 & $\frac{1}{Y}$ & 6.2561 & pass \\
\hline 2068 & 25.140 & 12.28 & 12.27 & 12.29 & 12.29 & 12.28 & 12.29 & $Y$ & 6.2603 & pass \\
\hline 2069 & 25.201 & 12.29 & 12.29 & 12.30 & 12.28 & 12.29 & 12.29 & $Y$ & 6.2533 & pass \\
\hline 2070 & 25.229 & 12.29 & 12.28 & 12.30 & 12.29 & 12.28 & 12.28 & $Y$ & 6.2581 & pass \\
\hline 2071 & 25.052 & 12.29 & 12.28 & 12.30 & 12.30 & 12.29 & 12.29 & $Y$ & 6.2468 & pass \\
\hline 2072 & 25.040 & 12.30 & 12.30 & 12.31 & 12.31 & 12.29 & 12.29 & $Y$ & 6.2603 & pass \\
\hline 2073 & 25.185 & 12.29 & 12.29 & 12.30 & 12.30 & 12.29 & 12.29 & $Y$ & 6.2516 & pass \\
\hline 2074 & 25.209 & 12.29 & 12.30 & 12.30 & 12.30 & 12.29 & 12.30 & $Y$ & 6.2532 & pass \\
\hline 2075 & 25.097 & 12.29 & 12.29 & 12.30 & 12.31 & 12.29 & 12.30 & $Y$ & 6.2532 & pass \\
\hline Z076 & 25.152 & 12.28 & 12.28 & 12.30 & 12.29 & 12.28 & 12.28 & $\bar{Y}$ & 6.2613 & pass \\
\hline 2077 & 25.255 & 12.29 & 12.29 & 12.30 & 12.29 & 12.28 & 12.29 & $Y$ & 6.2377 & pass \\
\hline 2078 & 25.242 & 12.30 & 12.30 & 12.30 & 12.31 & 12.29 & 12.29 & $y$ & 6.2327 & pass \\
\hline 2079 & 25.179 & 12.29 & 12.28 & 12.30 & 12.30 & 12.29 & 12.30 & $Y$ & 6.2446 & pass \\
\hline 2080 & 25.317 & 12.29 & 12.30 & 12.31 & 12.31 & 12.30 & 12.30 & $Y$ & 6.2702 & pass \\
\hline
\end{tabular}

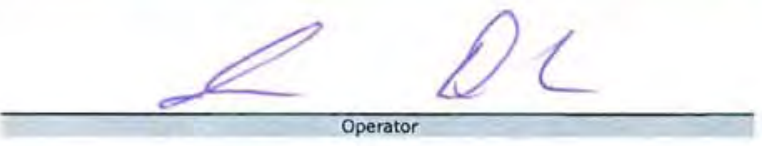

Lahn Mons

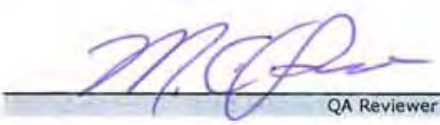

$2-27-09$

$7-24-09$

Date

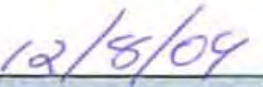


Data Report Form DRF-24A: Compact Diameter and Length

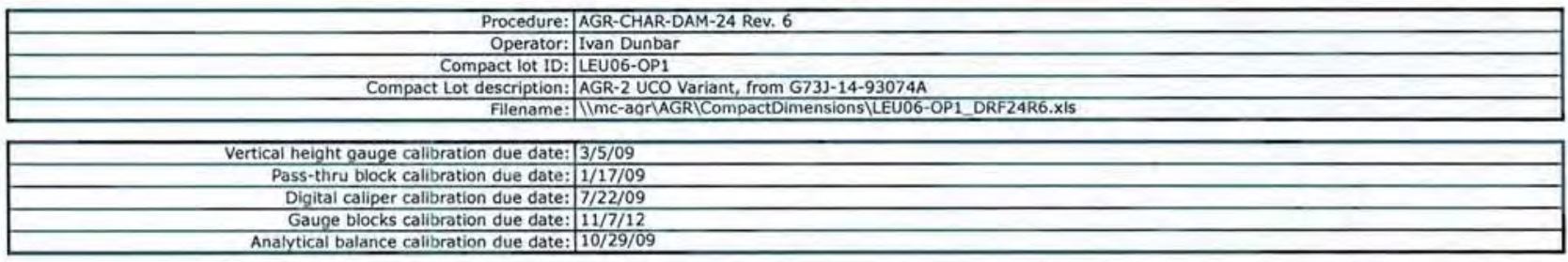

\begin{tabular}{|l|l|}
\hline Acceptance criteria for compact length: & 225.02 and $\leq 25.40 \mathrm{~mm}$ \\
\hline Acceptance criteria for compact diameter: & $\geq 12.22$ and $\leq 12.46 \mathrm{~mm}$ (and pass through $12.46 \mathrm{~mm}$ ring gauge) \\
\hline Acceptance criteria for compact mass: & For information only \\
\hline
\end{tabular}

\begin{tabular}{|c|c|c|c|c|c|c|c|c|c|c|}
\hline \multirow{2}{*}{$\begin{array}{c}\text { Compact } \\
\text { ID Number }\end{array}$} & \multirow{2}{*}{$\begin{array}{l}\text { Length } \\
(\mathrm{mm})\end{array}$} & \multicolumn{6}{|c|}{ Diameter $(\mathrm{mm})$} & \multirow{2}{*}{$\begin{array}{c}\text { Pass Thru? } \\
(\text { Y or } \mathrm{N})\end{array}$} & \multirow{2}{*}{$\begin{array}{l}\text { Compact weight } \\
\text { (a) }\end{array}$} & \multirow{2}{*}{$\begin{array}{c}\text { Accept? } \\
\text { (pass or fail) }\end{array}$} \\
\hline & & Top 1 & Top 2 & Middle 1 & Middle 2 & Bottom 1 & Bottom 2 & & & \\
\hline 2081 & & 12.30 & 12.30 & 12.30 & 12.30 & 12.30 & 12.30 & & & \\
\hline 2082 & 25.110 & 12.30 & 12.30 & 12.30 & 12.31 & 12.30 & 12.30 & $\bar{Y}$ & 6.2533 & pass \\
\hline 2083 & 25.318 & 12.29 & 12.29 & 12.29 & 12.29 & 12.28 & 12.28 & $\mathrm{Y}$ & 6.2374 & pass \\
\hline 2084 & 25.258 & 12.30 & 12.30 & 12.31 & 12.31 & 12.30 & 12.29 & $\bar{Y}$ & 6.2519 & pass \\
\hline 2085 & 25.157 & 12.30 & 12.29 & 12.32 & 12.31 & 12.30 & 12.30 & $\frac{Y}{Y}$ & 6.2596 & pass \\
\hline 2086 & 25.145 & 12.30 & 12.30 & 12.31 & 12.31 & 12.30 & 12.29 & $Y$ & 6.2504 & pass \\
\hline 2087 & 24.888 & 12.29 & 12.29 & 12.30 & 12.29 & 12.28 & 12.28 & $\frac{Y}{Y}$ & 6.2392 & fail \\
\hline 2088 & 25.183 & 12.29 & 12.29 & 12.31 & 12.30 & 12.29 & 12.29 & $Y$ & 6.2598 & pass \\
\hline 2089 & 25.185 & 12.29 & 12.29 & 12.30 & 12.30 & 12.29 & 12.29 & $Y$ & 6.2505 & pass \\
\hline 2090 & 25.145 & 12.30 & 12.30 & 12.30 & 12.30 & 12.30 & 12.30 & $Y$ & 6.2583 & pass \\
\hline 2091 & 25.312 & 12.30 & 12.30 & 12.31 & 12.30 & 12.29 & 12.29 & $Y$ & 6.2630 & pass \\
\hline 2092 & 25.140 & 12.31 & 12.30 & 12.31 & 12.30 & 12.31 & 12.31 & $\bar{Y}$ & 6.2565 & pass \\
\hline 2093 & 25.222 & 12.29 & 12.29 & 12.31 & 12.31 & 12.29 & 12.30 & $\bar{Y}$ & 6.2624 & pass \\
\hline 2094 & 25.231 & 12.29 & 12.29 & 12.30 & 12.30 & 12.30 & 12.29 & $Y$ & 6.2673 & pass \\
\hline 2095 & 25.052 & 12.29 & 12.30 & 12.30 & 12.30 & 12.29 & 12.28 & $Y$ & 6.2520 & pass \\
\hline 2096 & 25.031 & 12.29 & 12.30 & 12.30 & 12.31 & 12.29 & 12.28 & $\bar{Y}$ & 6.2423 & pass \\
\hline 2097 & 25.208 & 12.30 & 12.30 & 12.30 & 12.30 & 12.30 & 12.29 & $Y$ & 6.2401 & pass \\
\hline 2098 & 25.284 & 12.29 & 12.29 & 12.30 & 12.30 & 12.29 & 12.29 & $Y$ & 6.2372 & pass \\
\hline Z099 & 25.436 & 12.31 & 12.31 & 12.31 & 12.31 & 12.30 & 12.30 & $Y$ & 6.2695 & fall \\
\hline 2100 & 25.200 & 12.29 & 12.30 & 12.31 & 12.29 & 12.30 & 12.29 & $Y$ & 6.2560 & pass \\
\hline $\mathrm{Z101}$ & 25.130 & 12.31 & 12.31 & 12,31 & 12.31 & 12.31 & 12.31 & $Y$ & 6.2578 & pass \\
\hline 2102 & 25.055 & 12.30 & 12.29 & 12.30 & 12.30 & 12.30 & 12.30 & $\bar{Y}$ & 6.2366 & pass \\
\hline 2103 & 25.105 & 12.29 & 12.30 & 12.31 & 12.31 & 12.29 & 12.28 & $Y$ & 6.2580 & pass \\
\hline 2104 & 25.090 & 12.30 & 12.30 & 12.31 & 12.31 & 12.30 & 12.29 & $Y$ & 6.2679 & pass \\
\hline 2105 & 25.030 & 12.30 & 12.30 & 12.30 & 12.31 & 12.30 & 12.30 & $Y$ & 6.2612 & pass \\
\hline 2106 & 25.048 & 12.30 & 12.30 & 12.31 & 12.31 & 12.30 & 12.30 & $Y$ & 6.2478 & pass \\
\hline 2107 & 25.137 & 12,30 & 12.29 & 12.29 & 12.29 & 12.29 & 12.29 & $\bar{Y}$ & 6.2567 & pass \\
\hline Z108 & 25.361 & 12.30 & 12.29 & 12.31 & 12.30 & 12.29 & 12.29 & Y & 6.2392 & pass \\
\hline 2109 & $\frac{25.301}{25.154}$ & $\frac{12.30}{12.30}$ & $\frac{12.27}{12.30}$ & $\frac{12.31}{12.31}$ & 12.31 & 12.31 & 12.30 & $\frac{1}{Y}$ & $\frac{0.2592}{6.2475}$ & $\frac{p \text { pass }}{\text { pass }}$ \\
\hline $\mathrm{Z} 110$ & 25.261 & 12.29 & 12.28 & 12.30 & 12.30 & 12.29 & 12.29 & $Y$ & 6.2650 & pass \\
\hline Z111 & 25.152 & 12.29 & 12.28 & 12.28 & 12.28 & 12.28 & 12.28 & $y$ & 6.2692 & pass \\
\hline 2112 & 25.214 & 12.29 & 12.29 & 12.31 & 12.31 & 12.30 & 12.31 & $Y$ & 6.2469 & pass \\
\hline 2113 & 25.204 & 12.30 & 12.29 & 12.30 & 12.31 & 12.30 & 12.29 & $Y$ & 6.2511 & pass \\
\hline 2114 & 25.133 & 12.30 & 12.30 & 12.31 & 12.31 & 12.30 & 12.30 & $Y$ & 6.2580 & pass \\
\hline 2115 & 25.306 & 12.30 & 12.28 & 12.30 & 12.30 & 12.29 & 12.29 & $\bar{Y}$ & 6.2647 & pass \\
\hline Z116 & 25.236 & 12.29 & 12.29 & 12.30 & 12.30 & 12.29 & 12.29 & $Y$ & 6.2417 & pass \\
\hline 2117 & 25.213 & 12.28 & 12.29 & 12.30 & 12.30 & 12.28 & 12.29 & $Y$ & 6.2448 & pass \\
\hline 2118 & 25.161 & 12.30 & 12.30 & 12.31 & 12.31 & 12.30 & 12.30 & $\bar{Y}$ & 6.2392 & pass \\
\hline 2119 & 25.233 & 12.30 & 12.30 & 12.31 & 12.31 & 12.31 & 12.30 & $Y$ & 6.2487 & pass \\
\hline$Z 120$ & 25.130 & 12.30 & 12.31 & 12.29 & 12.30 & 12.30 & 12.29 & $Y$ & 6.2484 & pass \\
\hline
\end{tabular}

Compact LEU06-0P1-2087 was $0.132 \mathrm{~mm}$ below the lower limit on compact length; this comments

Compact LEU06-OP1-Z099 was 0.036
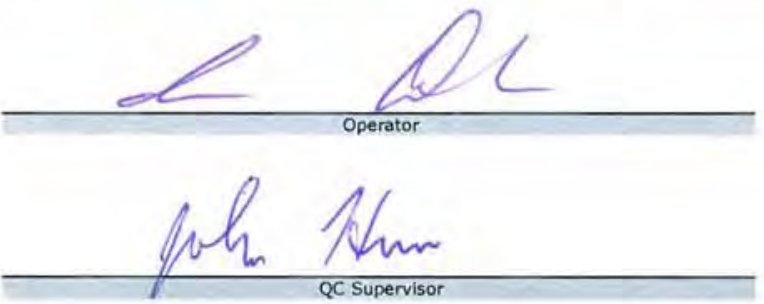

oncen-

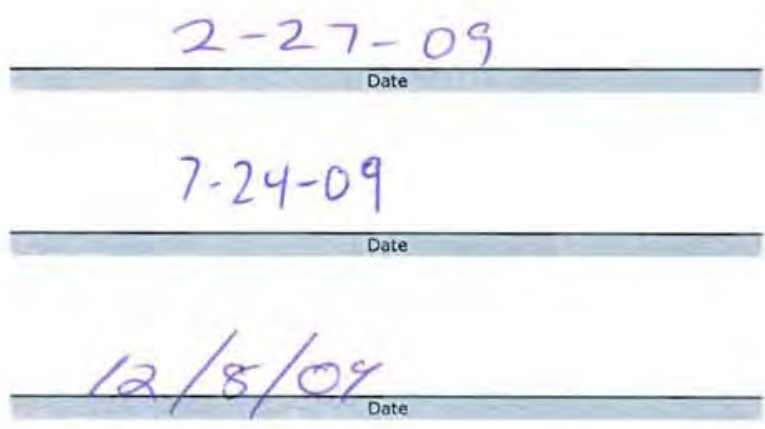


Data Report Form DRF-24A: Compact Diameter and Lenath

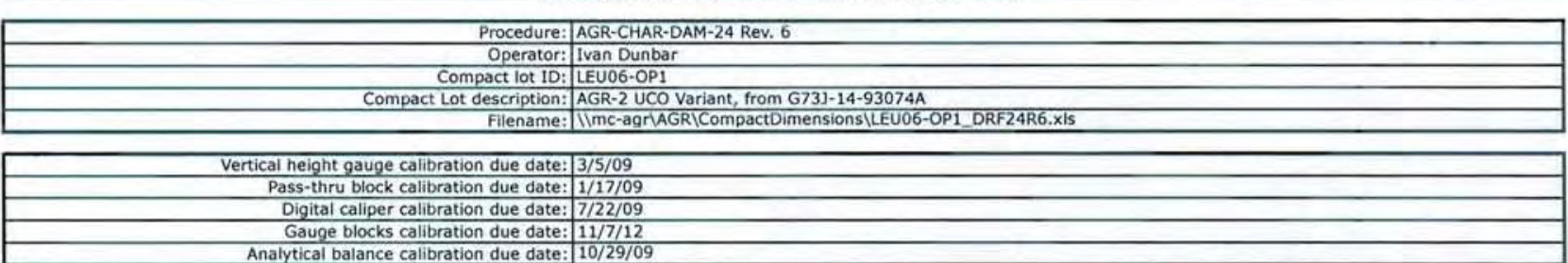

Acceptance criteria for compact length: $\quad \geq 25.02$ and $\$ 25.40 \mathrm{~mm}$

Acceptance criteria for compact diameter: $\geq 12.22$ and $\leq 12.46 \mathrm{~mm}$ (and pass through $12.46 \mathrm{~mm}$ ring gauge) Acceptance criteria for compact mass: For information only

\begin{tabular}{|c|c|c|c|c|c|c|c|c|c|c|}
\hline \multirow{2}{*}{$\begin{array}{l}\text { Compact } \\
\text { ID Number }\end{array}$} & \multirow{2}{*}{$\begin{array}{l}\text { Length } \\
\text { (mm) }\end{array}$} & \multicolumn{6}{|c|}{ Diameter $(\mathrm{mm})$} & \multirow{2}{*}{$\begin{array}{l}\text { Pass Thru? } \\
\text { (Y or N) }\end{array}$} & \multirow{2}{*}{\begin{tabular}{|c|} 
Compact weight \\
(a)
\end{tabular}} & \multirow{2}{*}{$\begin{array}{c}\text { Accept? } \\
\text { (pass or fail) }\end{array}$} \\
\hline & & Top 1 & Top 2 & Middle 1 & Middle 2 & Bottom 1 & Bottom 2 & & & \\
\hline 2121 & 25.065 & 12.28 & 12.28 & 12.30 & 12.30 & 12.29 & 12.29 & $Y$ & \begin{tabular}{|l|}
6.2379 \\
\end{tabular} & pass \\
\hline $\mathrm{Z} 122$ & 25.172 & 12.29 & 12.29 & 12.30 & 12.31 & 12.29 & 12.29 & $\bar{Y}$ & 6.2575 & pass \\
\hline$Z 123$ & 25.194 & 12.30 & 12.30 & 12.31 & 12.31 & 12.29 & 12.29 & $\bar{Y}$ & 6.2614 & pass \\
\hline 2124 & 25.192 & 12.29 & 12.29 & 12.30 & 12.30 & 12.29 & 12.29 & $Y$ & 6.2667 & pass \\
\hline 2125 & 25.193 & 12.30 & 12.29 & 12.30 & 12.30 & 12.30 & 12.29 & $Y$ & 6.2300 & pass \\
\hline$Z 126$ & 25.309 & 12.30 & 12.28 & 12.31 & 12.30 & 12.29 & 12.30 & $Y$ & 6.2488 & pass \\
\hline 2127 & 25.345 & 12.30 & 12.30 & 12.30 & 12.30 & 12.30 & 12.30 & $\bar{Y}$ & 6.2608 & pass \\
\hline$Z 128$ & 25.299 & 12.30 & 12.30 & 12.31 & 12.31 & 12.30 & 12.30 & $\bar{Y}$ & 6.2596 & pass \\
\hline 2129 & 25.154 & 12.30 & 12.30 & 12.31 & 12.32 & 12.31 & 12.31 & $\bar{Y}$ & 6.2658 & pass \\
\hline 2130 & 25.175 & 12.30 & 12.30 & 12.30 & 12.30 & 12.29 & 12.29 & $Y$ & 6.2507 & pass \\
\hline $\mathrm{Z131}$ & 25.040 & 12.28 & 12.29 & 12.31 & 12.30 & 12.30 & 12.30 & $Y$ & 6.2226 & pass \\
\hline$Z 132$ & 25.224 & 12.30 & 12.30 & 12.31 & 12.31 & 12.30 & 12.30 & $Y$ & 6.2618 & pass \\
\hline 2133 & 25.176 & 12.29 & 12.29 & 12.30 & 12.30 & 12.29 & 12.29 & $Y$ & 6.2496 & pass \\
\hline$Z 134$ & 25,325 & 12.29 & 12.29 & 12.30 & 12.30 & 12.30 & 12.29 & $Y$ & 6.2204 & pass \\
\hline Z135 & 25.199 & 12.28 & 12.28 & 12.29 & 12.29 & 12.29 & 12.29 & $Y$ & 6.2543 & pass \\
\hline \multicolumn{11}{|l|}{2136} \\
\hline \multicolumn{11}{|l|}{$Z 137$} \\
\hline \multirow{2}{*}{\multicolumn{11}{|c|}{$\mathrm{Z138}$}} \\
\hline \multirow{2}{*}{\multicolumn{11}{|c|}{$\begin{array}{l}Z 139 \\
Z 140\end{array}$}} \\
\hline & & & & & & & & & & \\
\hline \multicolumn{11}{|l|}{ Z141 } \\
\hline \multicolumn{11}{|l|}{$\mathrm{Z142}$} \\
\hline \multirow{2}{*}{\multicolumn{11}{|c|}{$\frac{Z 144}{Z 145}$}} \\
\hline & & & & & & & & & & \\
\hline \multicolumn{11}{|l|}{2146} \\
\hline \multicolumn{11}{|l|}{$Z 147$} \\
\hline \multicolumn{11}{|l|}{2148} \\
\hline \multicolumn{11}{|l|}{$\frac{2149}{7150}$} \\
\hline \multirow{2}{*}{\multicolumn{11}{|c|}{2151}} \\
\hline & & & & & & & & & & \\
\hline \multicolumn{11}{|l|}{ Z152 } \\
\hline \multicolumn{11}{|l|}{ Z153 } \\
\hline \multirow{2}{*}{\multicolumn{11}{|c|}{$\begin{array}{l}Z 154 \\
Z 155\end{array}$}} \\
\hline & & & & & & & & & & \\
\hline \multicolumn{11}{|l|}{2156} \\
\hline 2157 & & & & & & & & & & \\
\hline$Z 158$ & & & & & & & & & & \\
\hline 2159 & & & & & & & & & & \\
\hline 2160 & & & & & & & & & & \\
\hline
\end{tabular}

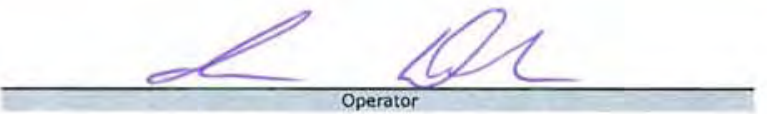

PNh ocsupervisor

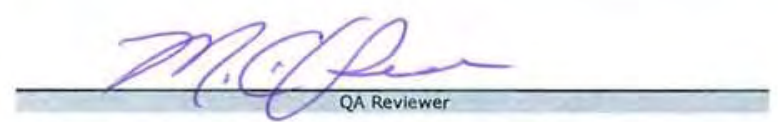

$$
2-27-09
$$

$$
7-24-09
$$

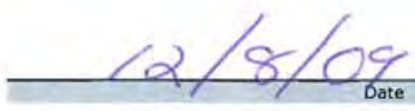


ORNL/TM-2010/296
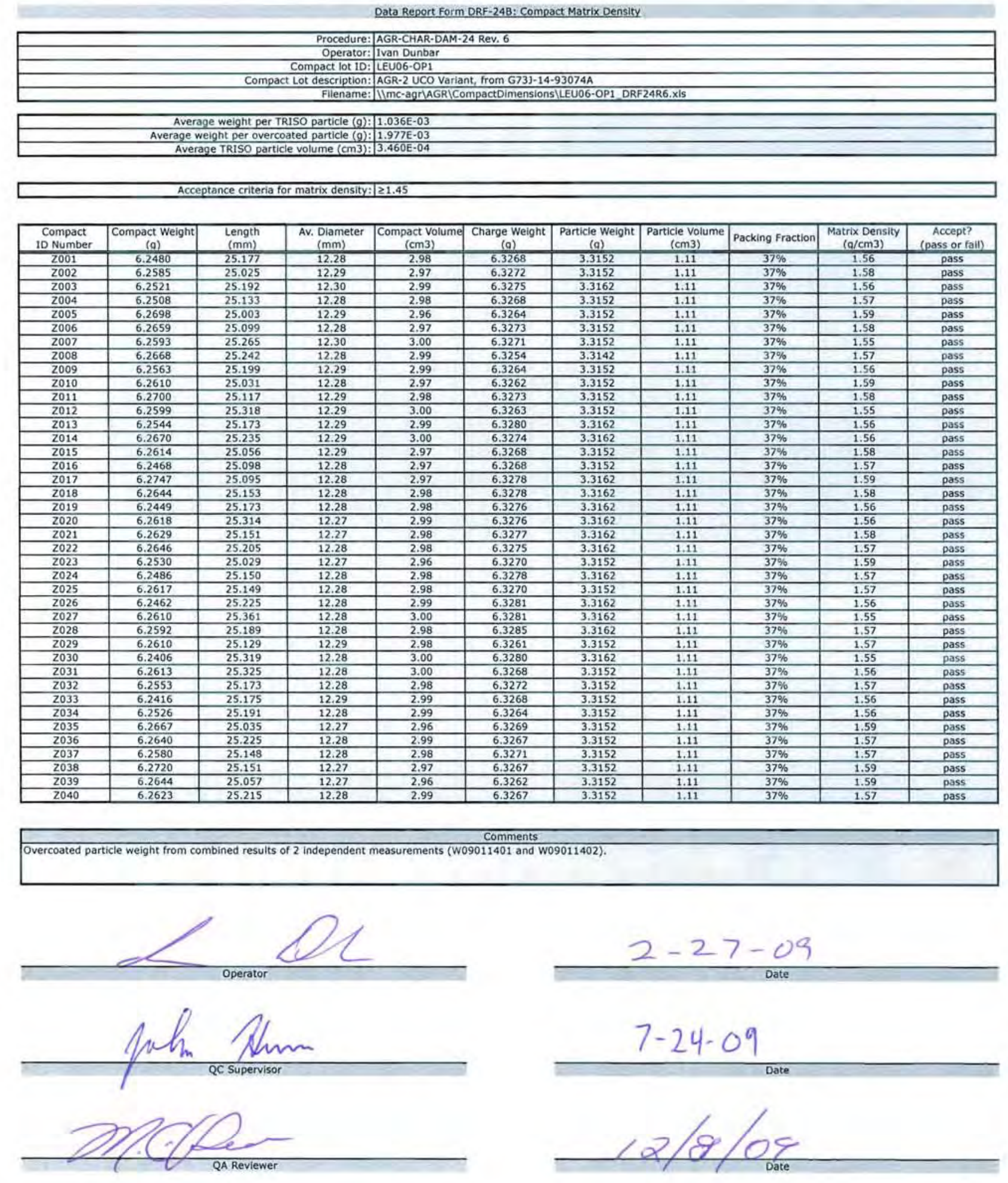

19 
Data Report Form DRF-248: Compact Matrix Density

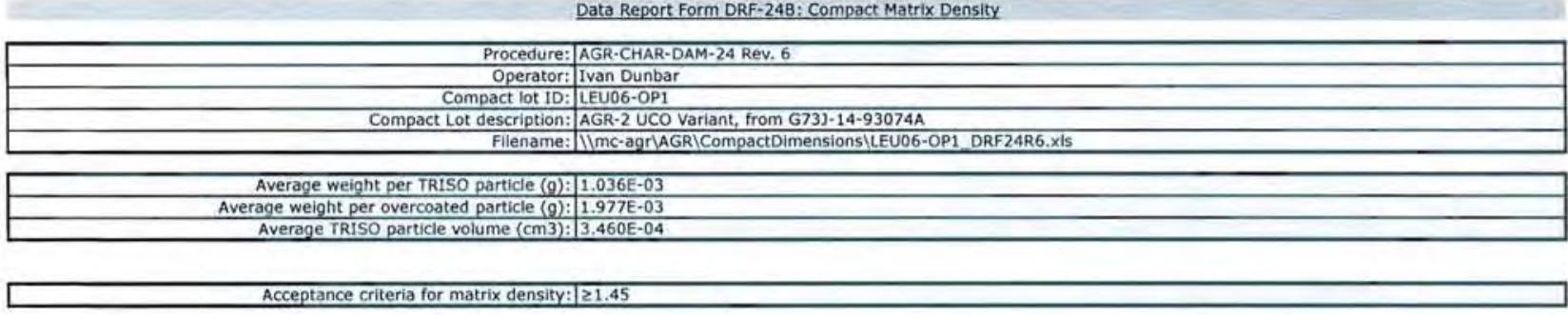

\begin{tabular}{|c|c|c|c|c|c|c|c|c|c|c|}
\hline $\begin{array}{l}\text { Compact } \\
\text { ID Number }\end{array}$ & $\begin{array}{l}\text { Compact Weight } \\
\text { (a) }\end{array}$ & $\begin{array}{l}\text { Length } \\
(\mathrm{mm})\end{array}$ & $\begin{array}{c}\text { Av. Diameter } \\
(\mathrm{mm})\end{array}$ & $\begin{array}{c}\text { Compact Volume } \\
(\mathrm{cm} 3)\end{array}$ & $\begin{array}{l}\text { Charge Weight } \\
\text { (a) }\end{array}$ & $\begin{array}{l}\text { Particle Weight } \\
\text { (a) }\end{array}$ & $\begin{array}{c}\text { Particle Volume } \\
(\mathrm{cm} 3)\end{array}$ & Packing Fraction & $\begin{array}{c}\text { Matrix Density } \\
(0 / \mathrm{cm} 3)\end{array}$ & $\begin{array}{c}\text { Accept? } \\
\text { (pass or fall) }\end{array}$ \\
\hline 2041 & 6.2570 & 25.263 & 12.28 & \begin{tabular}{l|l}
.99 \\
\end{tabular} & 6.3273 & 3.3152 & 1.11 & $37 \%$ & 1.56 & pass \\
\hline 2042 & 6.2592 & 25.248 & 12.28 & 2.99 & 6.3271 & 3.3152 & 1.11 & $37 \%$ & 1.56 & pass \\
\hline 2043 & 6.2391 & 25.059 & 12.28 & 2.97 & 6.3267 & 3.3152 & 1.11 & $37 \%$ & 1.57 & pass \\
\hline 2044 & 6.2207 & 25.015 & 12.28 & 2.96 & 6.3266 & 3.3152 & 1.11 & $37 \%$ & 1.57 & pass \\
\hline 2045 & 6.2484 & 25.111 & 12.27 & 2.97 & 6.3272 & 3.3152 & 1.11 & $37 \%$ & 1.57 & pass \\
\hline 2046 & 6.2633 & 25.236 & 12.27 & 2.98 & 6.3274 & 3.3162 & 1.11 & $37 \%$ & 1.57 & pass \\
\hline 2047 & 6.2569 & 25.305 & 12.27 & 2.99 & 6.3274 & 3.3162 & 1.11 & $37 \%$ & 1.56 & pass \\
\hline 2048 & 6.2583 & 25.180 & 12.29 & 2.99 & 6.3279 & 3.3162 & 1.11 & $37 \%$ & 1.57 & pass \\
\hline Z049 & 6.2775 & 25.340 & 12.28 & 3.00 & 6.3258 & 3.3152 & 1.11 & $37 \%$ & 1.56 & pass \\
\hline 2050 & 6.2359 & 25.262 & 12.28 & 2.99 & 6.3271 & 3.3152 & 1.11 & $37 \%$ & 1.55 & pass \\
\hline 2051 & 6.2435 & 25.133 & 12.27 & 2.97 & 6.3271 & 3.3152 & 1.11 & $37 \%$ & 1.57 & pass \\
\hline 2052 & 6.2631 & 25.205 & 12.27 & 2.98 & 6.3272 & 3.3152 & 1.11 & $37 \%$ & 1.57 & pass \\
\hline 2053 & $\frac{0.0312}{6.2432}$ & $\frac{25.205}{25.123}$ & $\frac{12.21}{12.28}$ & $\frac{2.96}{2.97}$ & $\frac{0.2572}{6.3276}$ & 3.3132 & 1.11 & $37 \%$ & 1.57 & $\begin{array}{l}\text { pass } \\
\text { pass }\end{array}$ \\
\hline 2054 & 6.2561 & 25.270 & 12.29 & 3.00 & 6.3279 & 3.3162 & 1.11 & $37 \%$ & 1.56 & pass \\
\hline 2055 & 6.2570 & 25.175 & 12.28 & 2.98 & 6.3270 & 3.3152 & 1.11 & $37 \%$ & 1.57 & pass \\
\hline 2056 & 6.2478 & 25.182 & 12.28 & 2.98 & 6.3268 & 3.3152 & 1.11 & $37 \%$ & 1.56 & pass \\
\hline 2057 & 6.2667 & 25.101 & 12.27 & 2.97 & 6.3273 & 3.3152 & 1.11 & $37 \%$ & 1.59 & pass \\
\hline 2058 & 6.2384 & 25.138 & 12.28 & 2.98 & 6.3270 & 3.3152 & 1.11 & $37 \%$ & 1.56 & pass \\
\hline 2059 & 6.2209 & 25.292 & 12.28 & 2.99 & 6.3260 & 3.3152 & 1.11 & $37 \%$ & 1.54 & pass \\
\hline 2060 & 6.2519 & 25.157 & 12.28 & 2.98 & 6.3279 & 3.3162 & 1.11 & $37 \%$ & 1.57 & pass \\
\hline 2061 & 6.2444 & 25.148 & 12.28 & 2.98 & 6.3272 & 3.3152 & 1.11 & $37 \%$ & 1.57 & pass \\
\hline 2062 & 6.2791 & 25.264 & 12.28 & 2.99 & 6.3263 & 3.3152 & 1.11 & $37 \%$ & 1.57 & pass \\
\hline 2063 & 6.2543 & 25.214 & 12.28 & 2.98 & 6.3274 & 3.3162 & 1.11 & $37 \%$ & 1.57 & pass \\
\hline 2064 & 6.2576 & 25.238 & 12.29 & 2.99 & 6.3272 & 3.3152 & 1.11 & $37 \%$ & 1.56 & pass \\
\hline 2065 & 6.2621 & 25.130 & 12.29 & 2.98 & 6.3270 & 3.3152 & 1.11 & $37 \%$ & 1.57 & pass \\
\hline 2066 & 6.2522 & 25.104 & 12.28 & 2.97 & 6.3262 & 3.3152 & 1.11 & $37 \%$ & 1.58 & pass \\
\hline 2067 & 6.2561 & 25.118 & 12.28 & 2.98 & 6.3270 & 3.3152 & 1.11 & $37 \%$ & 1.57 & pass \\
\hline 2068 & 6.2603 & 25.140 & 12.28 & 2.98 & 6.3264 & 3.3152 & 1.11 & $37 \%$ & 1.57 & pass \\
\hline 2069 & 6.2533 & 25.201 & 12.29 & 2.99 & 6.3279 & 3.3162 & 1.11 & $37 \%$ & 1.56 & pass \\
\hline 2070 & 6.2581 & 25.229 & 12.29 & 2.99 & 6.3260 & 3.3152 & 1.11 & $37 \%$ & 1.56 & pass \\
\hline 2071 & 6.2468 & 25.052 & 12.29 & 2.97 & 6.3267 & 3.3152 & 1.11 & $37 \%$ & 1.57 & pass \\
\hline 2072 & $\frac{0.460}{6.2603}$ & $\frac{3.032}{25.040}$ & 12.30 & $\frac{2.97}{2.98}$ & $\frac{0.3207}{6.3270}$ & $\frac{3.9132}{3.3152}$ & $\frac{1.11}{1.11}$ & $37 \%$ & 1.58 & pass \\
\hline 2073 & 6.2516 & 25.185 & 12.29 & 2.99 & 6.3270 & 3.3152 & 1.11 & $37 \%$ & 1.56 & pass \\
\hline 2074 & 6.2532 & 25.209 & 12.30 & 2.99 & 6.3271 & 3.3152 & 1.11 & $37 \%$ & 1.56 & pass \\
\hline 2075 & 6.2532 & 25.097 & 12.30 & 2.98 & 6.3279 & 3.3162 & 1.11 & $37 \%$ & 1.57 & pass \\
\hline 2076 & 6.2613 & 25.152 & 12.29 & 2.98 & 6.3273 & 3.3152 & 1.11 & $37 \%$ & 1.57 & pass \\
\hline 2077 & 6.2377 & 25.255 & 12.29 & 3.00 & 6.3263 & 3.3152 & 1.11 & $37 \%$ & 1.55 & pass \\
\hline 2078 & 6.2327 & 25.242 & 12.30 & 3.00 & 6.3282 & 3.3162 & 1.11 & $37 \%$ & 1.54 & pass \\
\hline 2079 & 6.2446 & 25.179 & 12.29 & 2.99 & 6.3282 & 3.3162 & 1.11 & $37 \%$ & 1.56 & pass \\
\hline 2080 & 6.2702 & 25.317 & 12.30 & 3.01 & 6.3278 & 3.3162 & 1.11 & $37 \%$ & 1.55 & pass \\
\hline
\end{tabular}

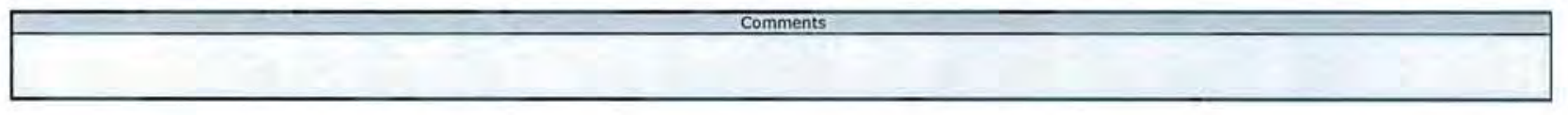

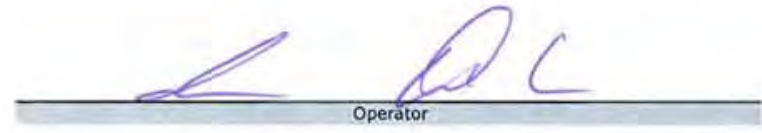
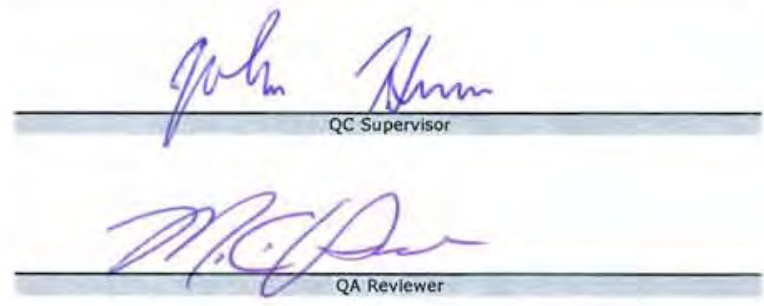

$\frac{2-27-09}{\text { Date }}$

$7-24-09$

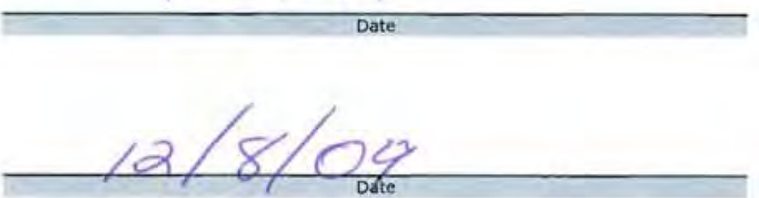


Data Report Form DRF-248: Compact Matrix Density

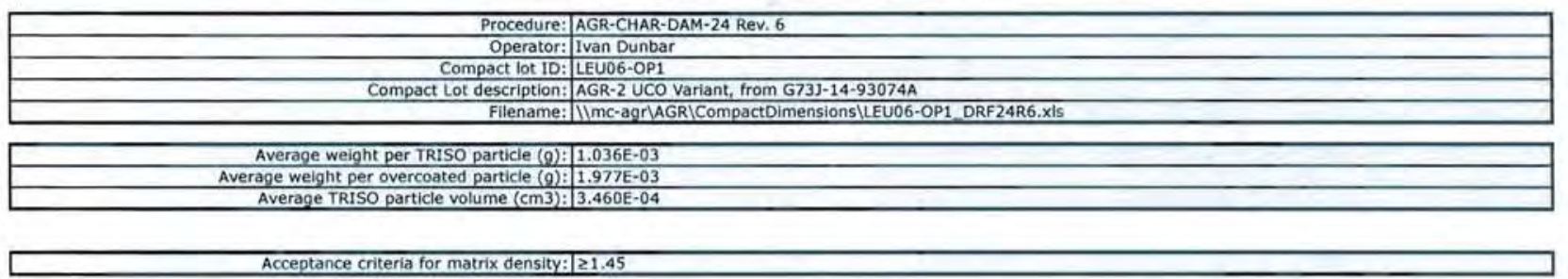

\begin{tabular}{|c|c|c|c|c|c|c|c|c|c|c|}
\hline $\begin{array}{l}\text { Compact } \\
\text { ID Number }\end{array}$ & $\begin{array}{l}\text { Compact Weight } \\
\text { (a) }\end{array}$ & $\begin{array}{l}\text { Length } \\
(\mathrm{mm})\end{array}$ & $\begin{array}{c}\text { Av. Diameter } \\
(\mathrm{mm})\end{array}$ & \begin{tabular}{|c|} 
Compact volume \\
$(\mathrm{cm} 3)$
\end{tabular} & $\begin{array}{l}\text { Charge Weight } \\
\text { (0) }\end{array}$ & $\begin{array}{l}\text { Particle Weight } \\
\text { (a) }\end{array}$ & \begin{tabular}{|c|} 
Particle Volume \\
$(\mathrm{cm} 3)$
\end{tabular} & Packing Fraction & $\begin{array}{c}\text { Matrix Density } \\
(\mathrm{a} / \mathrm{cm} 3)\end{array}$ & $\begin{array}{c}\text { Accept? } \\
\text { (pass or fail) }\end{array}$ \\
\hline 2081 & 6.2417 & 25.355 & 12.30 & 3.01 & 6.3276 & 3.3162 & $\begin{array}{l}1.11 \\
\end{array}$ & $37 \%$ & 1.54 & pass \\
\hline 2082 & 6.2533 & 25.110 & 12.30 & 2.98 & 6.3279 & 3.3162 & 1.11 & $37 \%$ & 1.56 & pass \\
\hline 2083 & 6.2374 & 25.318 & 12.29 & 3.00 & 6.3273 & 3.3152 & 1.11 & $37 \%$ & 1.54 & pass \\
\hline 2084 & 6.2519 & 25.258 & 12.30 & 3.00 & 6.3269 & 3.3152 & 1.11 & $37 \%$ & 1.55 & pass \\
\hline 2085 & 6.2596 & 25.157 & 12.30 & 2.99 & 6.3271 & 3.3152 & 1.11 & $37 \%$ & 1.56 & pass \\
\hline 2086 & 6.2504 & 25.145 & 12.30 & 2.99 & 6.3261 & 3.3152 & 1.11 & $37 \%$ & 1.56 & pass \\
\hline 2087 & 6.2392 & 24.888 & 12.29 & 2.95 & 6.3257 & 3.3152 & 1.11 & $38 \%$ & 1.59 & pass \\
\hline 2088 & 6.2598 & 25.183 & 12.30 & 2.99 & 6.3274 & 3.3162 & 1.11 & $37 \%$ & 1.56 & pass \\
\hline 2089 & 6.2505 & 25.185 & 12.29 & 2.99 & 6.3273 & 3.3152 & 1.11 & $37 \%$ & 1.56 & pass \\
\hline 2090 & 6.2583 & 25.145 & 12.30 & 2.99 & 6.3270 & 3.3152 & 1.11 & $37 \%$ & 1.56 & pass \\
\hline 2091 & 6.2630 & 25.312 & 12.30 & 3.01 & 6.3276 & 3.3162 & 1.11 & $37 \%$ & 1.55 & pass \\
\hline 2092 & 6.2565 & 25.140 & 12.31 & 2.99 & 6.3268 & 3.3152 & 1.11 & $37 \%$ & 1.56 & pass \\
\hline 2093 & 6.2624 & 25.222 & 12.30 & 3.00 & 6.3276 & 3.3162 & 1.11 & $37 \%$ & 1.56 & pass \\
\hline Z094 & $\frac{0.2024}{6.2673}$ & $\frac{23.222}{25.231}$ & $\frac{1.30}{12.30}$ & 3.00 & $\frac{0.3210}{6.3282}$ & $\frac{.30102}{3.3162}$ & $\frac{1.11}{1.11}$ & $37 \%$ & 1.56 & pass \\
\hline 2095 & 6.2520 & 25.052 & 12.29 & 2.97 & 6.3286 & 3.3162 & 1.11 & $37 \%$ & 1.57 & pass \\
\hline 2096 & 6.2423 & 25.031 & 12.30 & 2.97 & 6.3270 & 3.3152 & 1.11 & $37 \%$ & 1.57 & pass \\
\hline 2097 & 6.2401 & 25.208 & 12.30 & 2.99 & 6.3282 & 3.3162 & 1.11 & $37 \%$ & 1.55 & pass \\
\hline $\mathrm{Z} 098$ & 6.2372 & 25.284 & 12.29 & 3.00 & 6.3270 & 3.3152 & 1.11 & $37 \%$ & 1.54 & pass \\
\hline 2099 & 6.2695 & 25.436 & 12.31 & 3.03 & 6.3264 & 3.3152 & 1.11 & $37 \%$ & 1.54 & pass \\
\hline $\mathrm{Z} 100$ & 6.2560 & 25.200 & 12.30 & 2.99 & 6.3266 & 3.3152 & 1.11 & $37 \%$ & 1.56 & pass \\
\hline $\mathrm{Z101}$ & 6.2578 & 25.130 & 12.31 & 2.99 & 6.3271 & 3.3152 & 1.11 & $37 \%$ & 1.56 & pass \\
\hline $\mathrm{Z102}$ & 6.2366 & 25.055 & 12.30 & 2.98 & 6.3264 & 3.3152 & 1,11 & $37 \%$ & 1.56 & pass \\
\hline $\mathrm{Z} 103$ & 6.2580 & 25.105 & 12.30 & 2.98 & 6.3270 & 3.3152 & 1.11 & $37 \%$ & 1.57 & pass \\
\hline $\mathrm{Z104}$ & 6.2679 & 25.090 & 12.30 & 2.98 & 6.3270 & 3.3152 & 1.11 & $37 \%$ & 1.57 & pass \\
\hline $\mathrm{Z105}$ & 6.2612 & 25.030 & 12.30 & 2.97 & 6.3279 & 3.3162 & 1.11 & $37 \%$ & 1.58 & pass \\
\hline $\mathrm{Z} 106$ & 6.2478 & 25.048 & 12.30 & 2.98 & 6.3266 & 3.3152 & 1.11 & $37 \%$ & 1.57 & pass \\
\hline $\mathrm{Z} 107$ & 6.2567 & 25.137 & 12.29 & 2.98 & 6.3274 & 3.3162 & 1.11 & $37 \%$ & 1.57 & pass \\
\hline $\mathrm{Z} 108$ & 6.2392 & 25.361 & 12.30 & 3.01 & 6.3263 & 3.3152 & 1.11 & $37 \%$ & 1.54 & pass \\
\hline Z109 & 6.2475 & 25.154 & 12.31 & 2.99 & 6.3278 & 3.3162 & 1.11 & $37 \%$ & 1.56 & pass \\
\hline$\frac{2109}{\mathrm{z} 110}$ & $\frac{0.2413}{6.2650}$ & $\frac{25.54}{25.261}$ & $\frac{12.31}{12.29}$ & $\frac{1.97}{3.00}$ & $\frac{0.3270}{6.3275}$ & $\frac{3.302}{3.3162}$ & $\frac{1.11}{1.11}$ & $37 \%$ & 1.56 & pass \\
\hline $\mathrm{Z} 111$ & 6.2692 & 25.152 & 12.28 & 2.98 & 6.3272 & 3.3152 & 1.11 & $37 \%$ & 1.58 & pass \\
\hline $\mathrm{Z112}$ & 6.2469 & 25.214 & 12.30 & 3.00 & 6.3266 & 3.3152 & 1.11 & $37 \%$ & 1.55 & pass \\
\hline $\mathrm{Z} 113$ & 6.2511 & 25.204 & 12.30 & 2.99 & 6.3271 & 3.3152 & 1.11 & $37 \%$ & 1.56 & pass \\
\hline Z114 & 6.2580 & 25.133 & 12.30 & 2.99 & 6.3274 & 3.3162 & 1.11 & $37 \%$ & 1.56 & pass \\
\hline $\mathrm{Z} 115$ & 6.2647 & 25.306 & 12.29 & 3.00 & 6.3279 & 3.3162 & 1.11 & $37 \%$ & 1.55 & pass \\
\hline 2116 & 6.2417 & 25.236 & 12.29 & 3.00 & 6.3275 & 3.3162 & 1.11 & $37 \%$ & 1.55 & pass \\
\hline 2117 & 6.2448 & 25.213 & 12.29 & 2.99 & 6.3266 & 3.3152 & 1.11 & $37 \%$ & 1.56 & pass \\
\hline 2118 & 6.2392 & 25.161 & 12.30 & 2.99 & 6.3290 & 3.3162 & 1.11 & $37 \%$ & 1.55 & pass \\
\hline 2119 & 6.2487 & 25.233 & 12.31 & 3.00 & 6.3265 & 3.3152 & 1.11 & $37 \%$ & 1.55 & pass \\
\hline 2120 & 6.2484 & 25.130 & 12.30 & 2.99 & 6.3266 & 3.3152 & 1.11 & $37 \%$ & 1.56 & pass \\
\hline
\end{tabular}

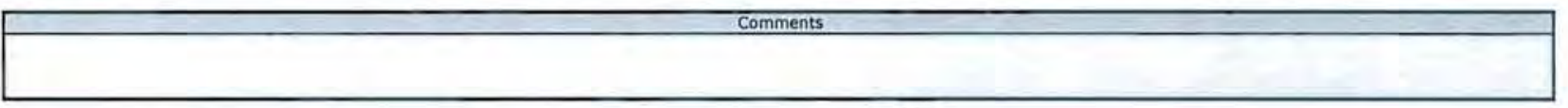

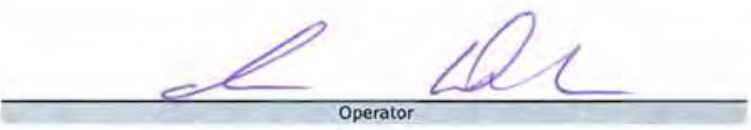
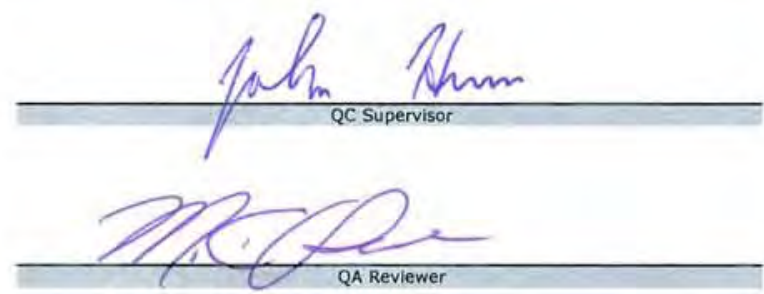

$$
2-27-09
$$

$7-24-09$

Date

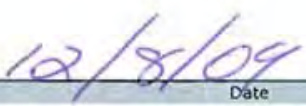


Data Report Form DRF-24B: Compact Matrix Density

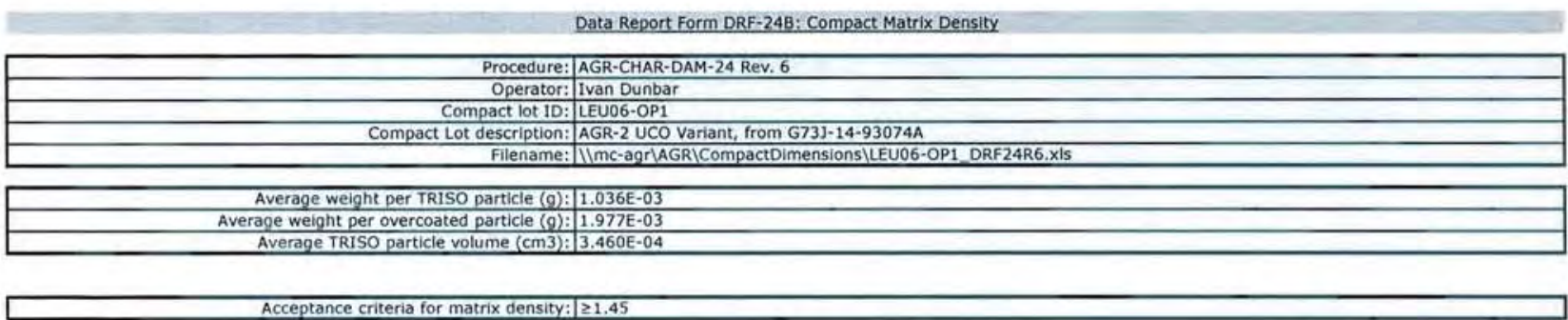

\begin{tabular}{|c|c|c|c|c|c|c|c|c|c|c|}
\hline $\begin{array}{l}\text { Compact } \\
\text { ID Number }\end{array}$ & $\begin{array}{l}\text { Compact Weight } \\
\text { (a) }\end{array}$ & $\begin{array}{l}\text { Length } \\
(\mathrm{mm})\end{array}$ & $\begin{array}{c}\text { Av. Dlameter } \\
(\mathrm{mm})\end{array}$ & $\begin{array}{c}\text { Compact volume } \\
(\mathrm{cm} 3)\end{array}$ & $\begin{array}{l}\text { Charge Weight } \\
\text { (a) }\end{array}$ & $\begin{array}{l}\text { Particle Weight } \\
\text { (a) }\end{array}$ & $\begin{array}{c}\text { Particle Volume } \\
(\mathrm{cm} 3)\end{array}$ & Packing Fraction & $\begin{array}{l}\text { Matrix Density } \\
(\mathrm{a} / \mathrm{cm} 3)\end{array}$ & $\begin{array}{c}\text { Accept? } \\
\text { (pass or fail) }\end{array}$ \\
\hline $\mathrm{Z121}$ & 6.2379 & 25.065 & 12.29 & 2.97 & 6.3275 & 3.3162 & 1.11 & $37 \%$ & 1.57 & pass \\
\hline 2122 & 6.2575 & 25.172 & 12.30 & 2.99 & 6.3278 & 3.3162 & 1.11 & $37 \%$ & 1.56 & pass \\
\hline $\mathrm{Z123}$ & 6.2614 & 25.194 & 12.30 & 2.99 & 6.3260 & 3.3152 & 1.11 & $37 \%$ & 1.56 & pass \\
\hline$Z 124$ & 6.2667 & 25.192 & $\frac{12.29}{1.70}$ & 2.99 & 6.3265 & 3.3152 & 1.11 & $37 \%$ & 1.57 & pass \\
\hline 2125 & 6.2300 & 25.193 & 12.30 & 2.99 & 6.3270 & 3.3152 & 1.11 & $37 \%$ & 1.55 & pass \\
\hline$Z 126$ & 6.2488 & 25.309 & 12.30 & 3.01 & 6.3281 & 3.3162 & 1.11 & $37 \%$ & 1.54 & pass \\
\hline$Z 127$ & 6.2608 & 25.345 & 12.30 & 3.01 & 6.3264 & 3.3152 & 1.11 & $37 \%$ & 1.55 & pass \\
\hline$Z 128$ & 6.2596 & 25.299 & 12.30 & 3.01 & 6.3272 & 3.3152 & 1.11 & $37 \%$ & 1.55 & pass \\
\hline 2129 & 6.2658 & 25.154 & 12.31 & 2.99 & 6.3267 & 3.3152 & 1.11 & $37 \%$ & 1.56 & pass \\
\hline$Z 130$ & 6.2507 & 25.175 & 12.30 & 2.99 & 6.3279 & 3.3162 & 1.11 & $37 \%$ & 1.56 & pass \\
\hline $\mathrm{Z131}$ & 6.2226 & 25.040 & 12.30 & 2.97 & 6.3270 & 3.3152 & 1.11 & $37 \%$ & 1.56 & pass \\
\hline 2132 & 6.2618 & 25.224 & 12.30 & 3.00 & 6.3279 & 3.3162 & 1.11 & $37 \%$ & 1.56 & pass \\
\hline 2133 & 6.2496 & 25.176 & 12.29 & 2.99 & 6.3287 & 3.3162 & 1.11 & $37 \%$ & 1.56 & pass \\
\hline Z134 & 6.2204 & 25.325 & 12.30 & 3.01 & 6.3276 & 3.3162 & 1.11 & $37 \%$ & 1.53 & pass \\
\hline 2135 & 6.2543 & 25.199 & 12.29 & 2.99 & 6.3279 & 3.3162 & 1.11 & $37 \%$ & 1.56 & pass \\
\hline \multirow{2}{*}{\multicolumn{11}{|c|}{2136}} \\
\hline \multirow{2}{*}{\multicolumn{11}{|c|}{$\frac{Z 137}{Z 138}$}} \\
\hline & & & & & & & & & & \\
\hline \multirow{2}{*}{\multicolumn{11}{|c|}{$\frac{2139}{7140}$}} \\
\hline & & & & & & & & & & \\
\hline \multicolumn{11}{|l|}{$\frac{1440}{Z 141}$} \\
\hline \multicolumn{11}{|l|}{2142} \\
\hline \multirow{2}{*}{\multicolumn{11}{|c|}{$\begin{array}{l}2143 \\
7144\end{array}$}} \\
\hline & & & & & & & & & & \\
\hline \multicolumn{11}{|l|}{$\frac{Z 144}{Z 145}$} \\
\hline \multirow{2}{*}{\multicolumn{11}{|c|}{2146}} \\
\hline \multirow{2}{*}{\multicolumn{11}{|c|}{$\frac{Z 147}{Z 148}$}} \\
\hline & & & & & & & & & & \\
\hline \multicolumn{11}{|l|}{2149} \\
\hline \multicolumn{11}{|l|}{2150} \\
\hline \multirow{2}{*}{\multicolumn{11}{|c|}{$\frac{Z 151}{Z 152}$}} \\
\hline & & & & & & & & & & \\
\hline \multicolumn{11}{|l|}{$\mathrm{Z} 153$} \\
\hline \multirow{2}{*}{\multicolumn{11}{|c|}{$\frac{Z 154}{Z 155}$}} \\
\hline & & & & & & & & & & \\
\hline \multicolumn{11}{|l|}{2156} \\
\hline \multirow{2}{*}{\multicolumn{11}{|c|}{$\frac{Z 157}{Z 158}$}} \\
\hline & & & & & & & & & & \\
\hline 2159 & & & & & & & & & & \\
\hline $\mathrm{Z160}$ & & & & & & & & & & \\
\hline
\end{tabular}

Comments

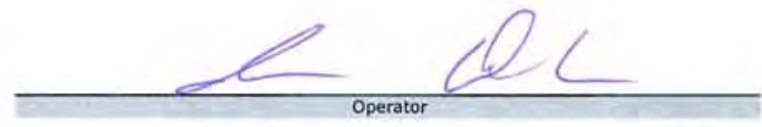

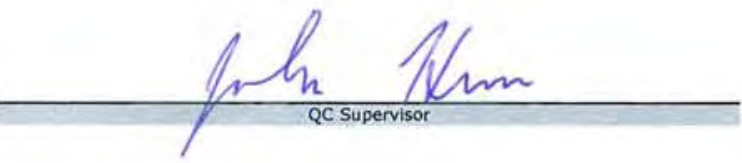

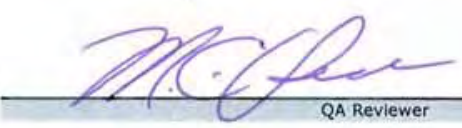

$2-27-09$

$7-24-09$

Date

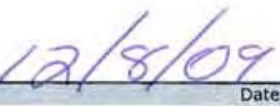


Data Report Form DRF-24C: Compact Tracking

\begin{tabular}{|r|l|}
\hline Procedure: & AGR-CHAR-DAM-24 Rev. 6 \\
\hline Operator: & Ivan Dunbar \\
\hline Compact lot ID: & LEU06-OP1 \\
\hline Compact Lot description: & AGR-2 UCO Variant, from G73J-14-93074A \\
\hline Filename: & IIme-agr\AGRICompactDimensions\LEU06-OP1 DRF24R6.xIS \\
\hline
\end{tabular}

\begin{tabular}{|c|c|}
\hline $\begin{array}{l}\text { Compact } \\
\text { Z Number }\end{array}$ & $\begin{array}{c}\text { Compact G } \\
\text { Number }\end{array}$ \\
\hline 2001 & $\mathrm{G} 043$ \\
\hline$Z 002$ & G165 \\
\hline$Z 003$ & G104 \\
\hline$Z 004$ & $\mathrm{G} 040$ \\
\hline Zo05 & G177 \\
\hline Z006 & G158 \\
\hline 2007 & G094 \\
\hline 2008 & G142 \\
\hline 2009 & G125 \\
\hline Z010 & G154 \\
\hline Z011 & G173 \\
\hline Z012 & G128 \\
\hline Z013 & G096 \\
\hline Z014 & G123 \\
\hline 2015 & G122 \\
\hline Z016 & G055 \\
\hline Z017 & G179 \\
\hline Z018 & G095 \\
\hline$Z 019$ & G037 \\
\hline 2020 & G087 \\
\hline Z021 & G159 \\
\hline Z022 & G075 \\
\hline Z023 & G083 \\
\hline 2024 & G109 \\
\hline Z025 & G066 \\
\hline Z026 & G036 \\
\hline 2027 & G117 \\
\hline Z028 & G099 \\
\hline 2029 & G129 \\
\hline 2030 & G008 \\
\hline Z031 & G118 \\
\hline Z032 & G126 \\
\hline Z033 & G110 \\
\hline Z034 & G124 \\
\hline Z035 & G162 \\
\hline 2036 & G130 \\
\hline Z037 & G127 \\
\hline Z038 & G176 \\
\hline Z039 & G161 \\
\hline ZO40 & G093 \\
\hline
\end{tabular}

\begin{tabular}{|c|c|}
\hline $\begin{array}{c}\text { Compact } \\
\text { Z Number }\end{array}$ & $\begin{array}{c}\text { Compact G } \\
\text { Number }\end{array}$ \\
\hline Z041 & G116 \\
\hline Z042 & G084 \\
\hline Z043 & G028 \\
\hline Z044 & G032 \\
\hline Z045 & G073 \\
\hline Z046 & G147 \\
\hline Z047 & G078 \\
\hline Z048 & G065 \\
\hline Z049 & G143 \\
\hline Z050 & G019 \\
\hline Z051 & G033 \\
\hline Z052 & G156 \\
\hline Z053 & G027 \\
\hline Z054 & G092 \\
\hline Z055 & G053 \\
\hline Z056 & G061 \\
\hline Z057 & G160 \\
\hline Z058 & G017 \\
\hline Z059 & G009 \\
\hline Z060 & G089 \\
\hline Z061 & G112 \\
\hline Z062 & G144 \\
\hline Z063 & G041 \\
\hline Z064 & G085 \\
\hline Z065 & G137 \\
\hline Z066 & G046 \\
\hline Z067 & G121 \\
\hline Z068 & G139 \\
\hline Z069 & G068 \\
\hline Z070 & G148 \\
\hline Z071 & G030 \\
\hline Z072 & G152 \\
\hline Z073 & G115 \\
\hline Z074 & G070 \\
\hline Z075 & G090 \\
\hline Z076 & G145 \\
\hline Z077 & G013 \\
\hline Z078 & G015 \\
\hline Z079 & G057 \\
\hline$Z 080$ & G100 \\
\hline
\end{tabular}

\begin{tabular}{|c|c|}
\hline $\begin{array}{c}\text { Compact } \\
\text { Z Number }\end{array}$ & $\begin{array}{c}\text { Compact G } \\
\text { Number }\end{array}$ \\
\hline Z081 & G011 \\
\hline Z082 & G067 \\
\hline Z083 & G007 \\
\hline Z084 & G105 \\
\hline Z085 & G134 \\
\hline Z086 & G131 \\
\hline Z087 & G164 \\
\hline Z088 & G074 \\
\hline Z089 & G051 \\
\hline Z090 & G101 \\
\hline Z091 & G080 \\
\hline Z092 & G133 \\
\hline Z093 & G077 \\
\hline Z094 & G006 \\
\hline Z095 & G047 \\
\hline Z096 & G114 \\
\hline Z097 & G016 \\
\hline Z098 & G005 \\
\hline Z099 & G098 \\
\hline Z100 & G049 \\
\hline Z101 & G064 \\
\hline Z102 & G060 \\
\hline Z103 & G153 \\
\hline Z104 & G163 \\
\hline Z105 & G171 \\
\hline Z106 & G108 \\
\hline Z107 & G097 \\
\hline Z108 & G021 \\
\hline Z109 & G111 \\
\hline Z110 & G138 \\
\hline Z111 & G172 \\
\hline Z112 & G062 \\
\hline Z113 & G052 \\
\hline Z114 & G132 \\
\hline Z115 & G076 \\
\hline Z116 & G014 \\
\hline Z117 & G038 \\
\hline Z118 & G004 \\
\hline Z119 & G091 \\
\hline$Z 120 ~$ & G069 \\
\hline
\end{tabular}

\begin{tabular}{|c|c|}
\hline $\begin{array}{l}\text { Compact } \\
\text { Z Number }\end{array}$ & $\begin{array}{c}\text { Compact G } \\
\text { Number }\end{array}$ \\
\hline Z121 & G034 \\
\hline $\mathrm{Z} 122$ & G072 \\
\hline Z123 & G135 \\
\hline Z124 & G149 \\
\hline $\mathrm{Z} 125$ & G018 \\
\hline Z126 & G113 \\
\hline Z127 & G136 \\
\hline $\mathrm{Z} 128$ & G119 \\
\hline Z129 & G141 \\
\hline Z130 & G039 \\
\hline Z131 & G035 \\
\hline $\mathrm{Z} 132$ & G086 \\
\hline $\mathrm{Z} 133$ & G058 \\
\hline Z134 & G010 \\
\hline Z135 & G103 \\
\hline Z136 & \\
\hline Z137 & \\
\hline Z138 & \\
\hline Z139 & \\
\hline Z140 & \\
\hline Z141 & \\
\hline Z142 & \\
\hline Z143 & \\
\hline$Z 144$ & \\
\hline$Z 145$ & \\
\hline Z146 & \\
\hline Z147 & \\
\hline Z148 & \\
\hline Z149 & \\
\hline$Z 150$ & \\
\hline$Z 151$ & \\
\hline Z152 & \\
\hline $\mathrm{Z} 153$ & \\
\hline Z154 & \\
\hline Z155 & \\
\hline$Z 156$ & \\
\hline Z157 & \\
\hline Z158 & \\
\hline Z159 & \\
\hline Z160 & \\
\hline
\end{tabular}

Comments

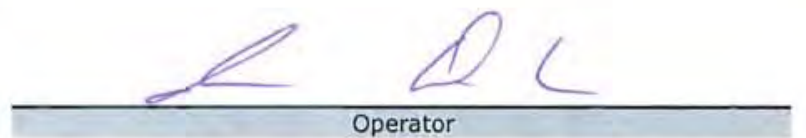

$2-16-09$

Operator

Date 
Data Report Form DRF-24D: Compact Charge Weight

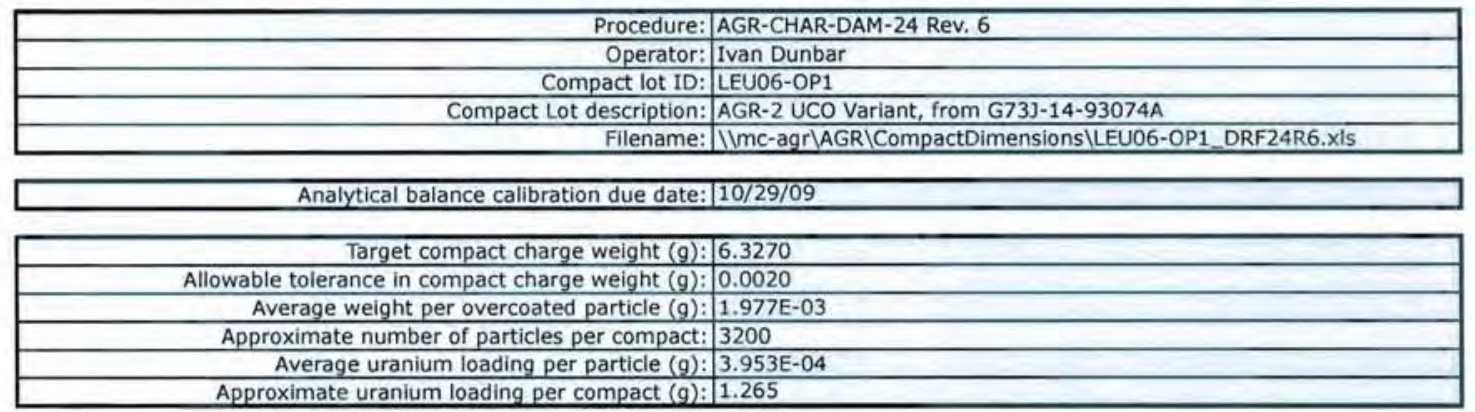

\begin{tabular}{|c|c|}
\hline $\begin{array}{l}\text { Compact } \\
\text { G Number }\end{array}$ & \begin{tabular}{|c|} 
Charge Weight \\
(g) \\
\end{tabular} \\
\hline G001 & 6.3272 \\
\hline $\mathrm{GOO2}$ & 6.3280 \\
\hline G003 & 6.3274 \\
\hline G004 & 6.3290 \\
\hline G005 & 6.3270 \\
\hline G006 & 6.3282 \\
\hline G007 & 6.3273 \\
\hline G008 & 6.3280 \\
\hline G009 & 6.3260 \\
\hline G010 & 6.3276 \\
\hline G011 & 6.3276 \\
\hline G012 & 6.3264 \\
\hline G013 & 6.3263 \\
\hline G014 & 6.3275 \\
\hline G015 & 6.3282 \\
\hline G016 & 6.3282 \\
\hline G017 & 6.3270 \\
\hline G018 & 6.3270 \\
\hline G019 & 6.3271 \\
\hline G020 & 6.3278 \\
\hline G021 & 6.3263 \\
\hline G022 & 6.3252 \\
\hline G023 & 6.3282 \\
\hline G024 & 6.3272 \\
\hline $\mathrm{G} 025$ & 6.3283 \\
\hline G026 & 6.3286 \\
\hline $\mathrm{G} 027$ & 6.3276 \\
\hline G028 & 6.3267 \\
\hline G029 & 6.3261 \\
\hline G030 & 6.3267 \\
\hline G031 & 6.3266 \\
\hline G032 & 6.3266 \\
\hline G033 & 6.3271 \\
\hline G034 & 6.3275 \\
\hline G035 & 6.3270 \\
\hline G036 & 6.3281 \\
\hline G037 & 6.3276 \\
\hline G038 & 6.3266 \\
\hline G039 & 6.3279 \\
\hline G040 & 6.3268 \\
\hline G041 & 6.3274 \\
\hline G042 & 6.3268 \\
\hline $\mathrm{G} 043$ & 6.3268 \\
\hline G044 & 6.3269 \\
\hline G045 & 6.3284 \\
\hline
\end{tabular}

\begin{tabular}{|c|c|}
\hline $\begin{array}{c}\text { Compact } \\
\text { G Number }\end{array}$ & $\begin{array}{c}\text { Charge Weight } \\
(\mathrm{g})\end{array}$ \\
\hline G046 & 6.3262 \\
\hline G047 & 6.3286 \\
\hline G048 & 6.3267 \\
\hline G049 & 6.3266 \\
\hline G050 & 6.3275 \\
\hline G051 & 6.3273 \\
\hline G052 & 6.3271 \\
\hline G053 & 6.3270 \\
\hline G054 & 6.3277 \\
\hline G055 & 6.3268 \\
\hline G056 & 6.3286 \\
\hline G057 & 6.3282 \\
\hline G058 & 6.3287 \\
\hline G059 & 6.3266 \\
\hline G060 & 6.3264 \\
\hline G061 & 6.3268 \\
\hline G062 & 6.3266 \\
\hline G063 & 6.3278 \\
\hline G064 & 6.3271 \\
\hline G065 & 6.3279 \\
\hline G066 & 6.3270 \\
\hline G067 & 6.3279 \\
\hline G068 & 6.3279 \\
\hline G069 & 6.3266 \\
\hline G070 & 6.3271 \\
\hline G071 & 6.3269 \\
\hline G072 & 6.3278 \\
\hline G073 & 6.3272 \\
\hline G074 & 6.3274 \\
\hline G075 & 6.3275 \\
\hline G076 & 6.3279 \\
\hline G077 & 6.3276 \\
\hline G078 & 6.3274 \\
\hline G079 & 6.3273 \\
\hline G080 & 6.3276 \\
\hline G081 & 6.3281 \\
\hline G082 & 6.3276 \\
\hline G083 & 6.3270 \\
\hline G086 & 6.3271 \\
\hline & 6.3272 \\
\hline & 6.3279 \\
\hline 6.3276 \\
\hline
\end{tabular}

\begin{tabular}{|c|c|}
\hline $\begin{array}{c}\text { Compact } \\
\text { G Number }\end{array}$ & $\begin{array}{c}\text { Charge Weight } \\
(\mathrm{g})\end{array}$ \\
\hline G091 & 6.3265 \\
\hline G092 & 6.3279 \\
\hline G093 & 6.3267 \\
\hline G094 & 6.3271 \\
\hline G095 & 6.3278 \\
\hline G096 & 6.3280 \\
\hline G097 & 6.3274 \\
\hline G098 & 6.3264 \\
\hline G099 & 6.3285 \\
\hline G100 & 6.3278 \\
\hline G101 & 6.3270 \\
\hline G102 & 6.3274 \\
\hline G103 & 6.3279 \\
\hline G104 & 6.3275 \\
\hline G105 & 6.3269 \\
\hline G106 & 6.3272 \\
\hline G107 & 6.3281 \\
\hline G108 & 6.3266 \\
\hline G109 & 6.3278 \\
\hline G110 & 6.3268 \\
\hline G111 & 6.3278 \\
\hline G112 & 6.3272 \\
\hline G113 & 6.3281 \\
\hline G114 & 6.3270 \\
\hline G115 & 6.3270 \\
\hline G116 & 6.3273 \\
\hline G117 & 6.3281 \\
\hline G118 & 6.3268 \\
\hline G119 & 6.3272 \\
\hline G120 & 6.3276 \\
\hline G121 & 6.3270 \\
\hline G122 & 6.3268 \\
\hline G123 & 6.3274 \\
\hline G124 & 6.3264 \\
\hline G125 & 6.3264 \\
\hline G126 & 6.3272 \\
\hline G127 & 6.3271 \\
\hline G128 & 6.3263 \\
\hline G130 & 6.3261 \\
\hline G13133 & 6.3267 \\
\hline & 6.3261 \\
\hline & 6.3274 \\
\hline G135 & 6.3268 \\
\hline & 6.3260 \\
\hline
\end{tabular}

\begin{tabular}{|c|c|}
\hline $\begin{array}{c}\text { Compact } \\
\text { G Number }\end{array}$ & $\begin{array}{c}\text { Charge Weigh } \\
\text { (9) }\end{array}$ \\
\hline G136 & 6.3264 \\
\hline G137 & 6.3270 \\
\hline G138 & 6.3275 \\
\hline G139 & 6.3264 \\
\hline G140 & 6.3275 \\
\hline G141 & 6.3267 \\
\hline G142 & 6.3254 \\
\hline G143 & 6.3258 \\
\hline G144 & 6.3263 \\
\hline G145 & 6.3273 \\
\hline G146 & 6.3262 \\
\hline G147 & 6.3274 \\
\hline G148 & 6.3260 \\
\hline G149 & 6.3265 \\
\hline G150 & 6.3269 \\
\hline G151 & 6.3276 \\
\hline G152 & 6.3270 \\
\hline G153 & 6.3270 \\
\hline G154 & 6.3262 \\
\hline G155 & 6.3279 \\
\hline G156 & 6.3272 \\
\hline G157 & 6.3264 \\
\hline G158 & 6.3273 \\
\hline G159 & 6.3277 \\
\hline G160 & 6.3273 \\
\hline G161 & 6.3262 \\
\hline G162 & 6.3269 \\
\hline G163 & 6.3270 \\
\hline G164 & 6.3257 \\
\hline G165 & 6.3272 \\
\hline G166 & 6.3264 \\
\hline G167 & 6.3280 \\
\hline G169 & 6.3266 \\
\hline G170 & 6.3269 \\
\hline G171 & 6.3261 \\
\hline G172 & 6.3279 \\
\hline G173 & 6.3272 \\
\hline G175 & 6.3273 \\
\hline G177 & 6.3270 \\
\hline & 6.3278 \\
\hline & 6.3267 \\
\hline & 6.3264 \\
\hline & 6.3270 \\
\hline & 6.3270 \\
\hline
\end{tabular}

Comments

Overcoated particle weight from combined results of 2 independent measurements (W09011401 and W09011402).

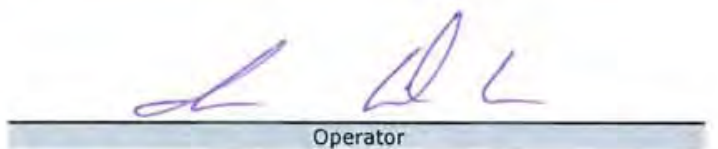

$$
2-4-09
$$


Data Report Form DRF-24A: Compact Dlameter and Length

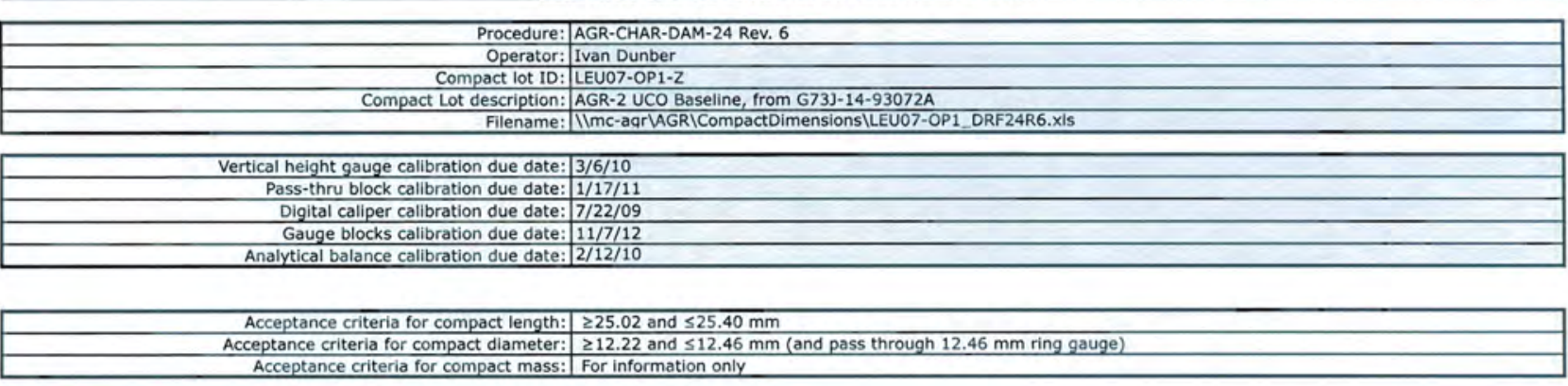

\begin{tabular}{|c|c|c|c|c|c|c|c|c|c|c|}
\hline \multirow{2}{*}{$\begin{array}{l}\text { Compact } \\
\text { ID Number }\end{array}$} & \multirow{2}{*}{$\begin{array}{l}\text { Length } \\
(\mathrm{mm})\end{array}$} & \multicolumn{6}{|c|}{ Diameter $(\mathrm{mm})$} & \multirow{2}{*}{$\begin{array}{l}\text { Pass Thru? } \\
\text { (Yor } \mathrm{N})\end{array}$} & \multirow{2}{*}{$\begin{array}{l}\text { Compact weight } \\
\text { (a) }\end{array}$} & \multirow{2}{*}{$\begin{array}{c}\text { Accept? } \\
\text { (pass or fail) }\end{array}$} \\
\hline & & Top 1 & Top 2 & Middle 1 & Middle 2 & Bottom 1 & Bottom 2 & & & \\
\hline & & 12.31 & 12.31 & 12.32 & 12.32 & 12.31 & 12.31 & & & \\
\hline 2002 & 25.284 & 12.31 & 12.31 & 12.32 & 12.32 & 12.31 & 12.31 & $Y$ & 6.3501 & pass \\
\hline 2003 & 25.159 & 12.31 & 12.31 & 12.32 & 12.32 & 12.32 & 12.32 & $\begin{array}{r}\mathrm{Y} \\
\end{array}$ & 6.3504 & pass \\
\hline 2004 & 25.124 & 12.31 & 12.30 & 12.32 & 12.32 & 12.31 & 12.31 & $\bar{Y}$ & 6.3500 & pass \\
\hline 2005 & 25.218 & 12.31 & 12.31 & 12.32 & 12.32 & 12.31 & 12.31 & $\bar{Y}$ & 6.3466 & pass \\
\hline 2006 & 25.225 & 12.31 & 12.31 & 12.33 & 12.32 & 12.32 & 12.32 & $\bar{Y}$ & 6.3508 & pass \\
\hline 2007 & 25.242 & 12.31 & 12.31 & 12.32 & 12.32 & 12.31 & 12.32 & $\mathrm{Y}$ & 6.3453 & pass \\
\hline 2008 & 25.197 & 12.32 & 12.33 & 12.33 & 12.34 & 12.33 & 12.33 & $r$ & 6.3609 & pass \\
\hline 2009 & 25.165 & 12.30 & 12.31 & 12.32 & 12.32 & 12.31 & 12.31 & $\mathrm{Y}$ & 6.3542 & pass \\
\hline 2010 & 25.204 & 12.31 & 12.31 & 12.32 & 12.32 & 12.31 & 12.30 & $\mathrm{Y}$ & 6.3419 & pass \\
\hline 2011 & 25.144 & 12.31 & 12.31 & 12.32 & 12.32 & 12.30 & 12.30 & $Y$ & 6.3376 & pass \\
\hline 2012 & 25.358 & 12.31 & 12.30 & 12.31 & 12.31 & 12.30 & 12.31 & $Y$ & 6.3532 & pass \\
\hline 2013 & 25.217 & 12.30 & 12.30 & 12.32 & 12.32 & 12.31 & 12.31 & $Y$ & 6.3393 & pass \\
\hline 2014 & 25.212 & 12.30 & 12.31 & 12.32 & 12.32 & 12.31 & 12.31 & $Y$ & 6.3355 & pass \\
\hline 2015 & 25.352 & 12.32 & 12.32 & 12.33 & 12.33 & 12.31 & 12.31 & $\bar{Y}$ & 6.3399 & pass \\
\hline 2016 & 25.232 & 12.32 & 12.31 & 12.33 & 12.33 & 12.31 & 12.31 & $\bar{Y}$ & 6.3613 & pass \\
\hline 2017 & 25.063 & 12.31 & 12.31 & 12.32 & 12.31 & 12.30 & 12.30 & $Y$ & 6.3530 & pass \\
\hline 2018 & 25.172 & 12.31 & 12.31 & 12.32 & 12.31 & 12.30 & 12.30 & $Y$ & 6.3494 & pass \\
\hline Z019 & 25.215 & 12.31 & 12.30 & 12.32 & 12.32 & 12.30 & 12.30 & $Y$ & 6.3473 & pass \\
\hline 2020 & 25.232 & 12.31 & 12.31 & 12.33 & 12.33 & 12.31 & 12.31 & $\bar{Y}$ & 6.3604 & pass \\
\hline 2021 & 25.073 & 12.31 & 12.30 & 12.31 & 12.32 & 12.30 & 12.31 & $Y$ & 6.3369 & pass \\
\hline 2022 & 25.222 & 12.31 & 12.31 & 12.32 & 12.32 & 12.30 & 12.30 & $Y$ & 6.3438 & pass \\
\hline 2023 & 25.152 & 12.30 & 12.31 & 12.32 & 12.32 & 12.30 & 12.30 & $Y$ & 6.3443 & pass \\
\hline 2024 & 25.151 & 12.31 & 12.30 & 12.32 & 12.32 & 12.30 & 12.31 & $Y$ & 6.3502 & pass \\
\hline 2025 & 25.112 & 12.31 & 12.31 & 12.32 & 12.31 & 12.32 & 12.32 & $\mathrm{Y}$ & 6.3495 & pass \\
\hline 2026 & 25.196 & 12.31 & 12.31 & 12.32 & 12.32 & 12.31 & 12.31 & $\bar{Y}$ & 6.3415 & pass \\
\hline 2027 & 25.233 & 12.31 & 12.30 & 12.32 & 12.32 & 12.31 & 12.31 & $Y$ & 6.3492 & pass \\
\hline 2028 & 25.134 & 12.31 & 12.31 & 12.32 & 12.32 & 12.31 & 12.31 & $Y$ & 6.3530 & pass \\
\hline 2029 & 25.093 & 12.30 & 12.30 & 12.31 & 12.31 & 12.30 & 12.30 & $Y$ & 6.3447 & pass \\
\hline 2030 & 25.156 & 12.30 & 12.30 & 12.31 & 12.31 & 12.30 & 12.30 & $Y$ & 6.3453 & pass \\
\hline Z031 & 25.134 & 12.30 & 12.30 & 12.31 & 12.31 & 12.30 & 12.30 & $Y$ & 6.3499 & pass \\
\hline 2032 & 25.201 & 12.32 & 12.32 & 12.33 & 12.33 & 12.32 & 12.32 & $Y$ & 6.3590 & pass \\
\hline 2033 & 25.093 & 12.31 & 12.31 & 12.32 & 12.32 & 12.31 & 12.31 & $Y$ & 6.3267 & pass \\
\hline 2034 & 25.221 & 12.32 & 12.32 & 12.32 & 12.32 & 12.31 & 12.32 & $Y$ & 6.3420 & pass \\
\hline 2035 & 25.303 & 12.30 & 12.30 & 12.32 & 12.32 & 12.30 & 12.30 & $Y$ & 6.3611 & pass \\
\hline 2036 & 25.224 & 12.30 & 12.30 & 12.31 & 12.31 & 12.30 & 12.30 & $Y$ & 6.3464 & pass \\
\hline 2037 & 25.134 & 12.30 & 12.30 & 12.31 & 12.32 & 12.31 & 12.30 & $Y$ & 6.3399 & pass \\
\hline 2038 & 25.132 & 12.31 & 12.31 & 12.32 & 12.32 & 12.31 & 12.31 & $Y$ & 6.3407 & pass \\
\hline 2039 & 25.185 & 12.31 & 12.31 & 12.32 & 12.33 & 12.32 & 12.31 & $Y$ & 6.3449 & pass \\
\hline 2040 & 25.204 & 12.30 & 12.30 & 12.31 & 12.31 & 12.30 & 12.30 & $Y$ & 6.3496 & pass \\
\hline
\end{tabular}

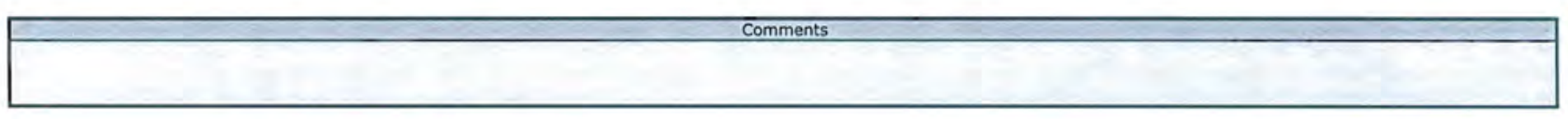

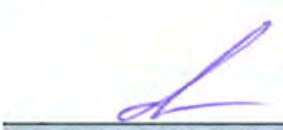
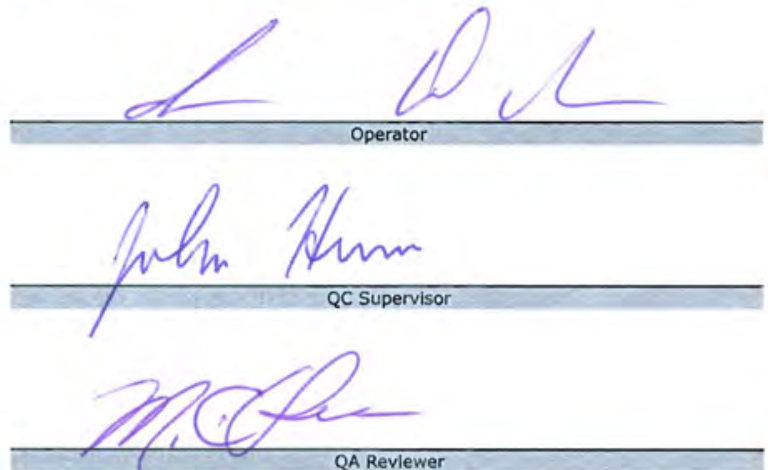

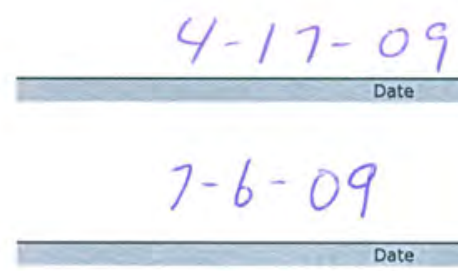

$12 / 8 / 29$ 
Data Report Form DRF-24A: Compact Diameter and Lenath

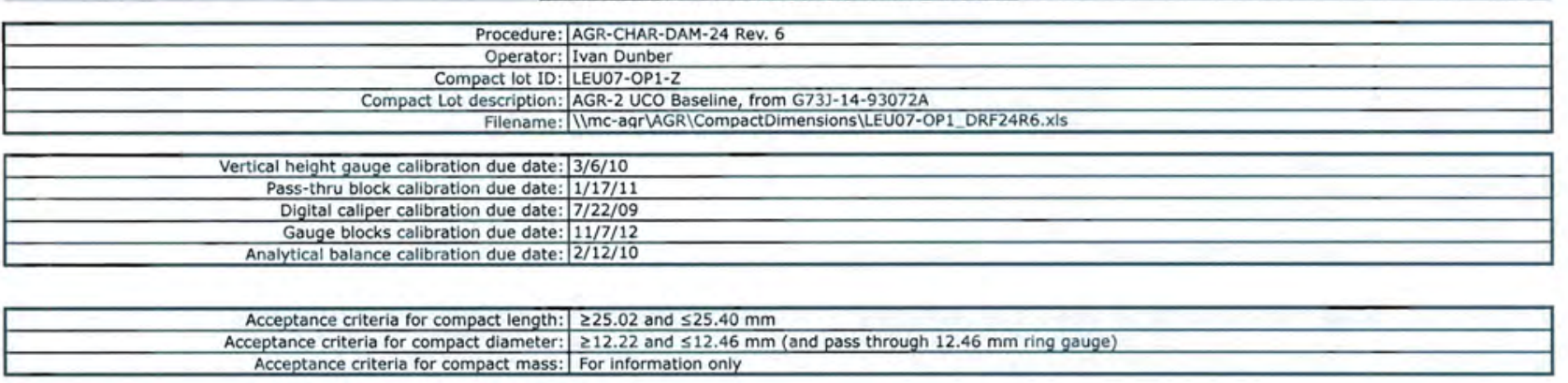

\begin{tabular}{|c|c|c|c|c|c|c|c|c|c|c|}
\hline \multirow{2}{*}{$\begin{array}{l}\text { Compact } \\
\text { ID Number }\end{array}$} & \multirow{2}{*}{$\begin{array}{l}\text { Length } \\
(\mathrm{mm})\end{array}$} & \multicolumn{6}{|c|}{ Diameter $(\mathrm{mm})$} & \multirow{2}{*}{$\begin{array}{c}\text { Pass Thru? } \\
(Y \text { or } N)\end{array}$} & \multirow{2}{*}{\begin{tabular}{l|} 
Compact weight \\
(a)
\end{tabular}} & \multirow{2}{*}{$\begin{array}{c}\text { Accept? } \\
\text { (pass or fail) }\end{array}$} \\
\hline & & Top 1 & Top 2 & Middle 1 & Middle 2 & Bottom 1 & Bottom 2 & & & \\
\hline 2041 & & 12.31 & 12.30 & 12.31 & 12.31 & 12.31 & 12.30 & & & \\
\hline 2042 & 25.226 & 12.31 & 12.31 & 12.32 & 12.33 & 12.32 & 12.31 & $\mathrm{Y}$ & 6.3584 & pass \\
\hline 2043 & 25.063 & 12.31 & 12.30 & 12.32 & 12.31 & 12.31 & 12.31 & $\begin{array}{r}\mathrm{Y} \\
\mathrm{y}\end{array}$ & 6.3437 & pass \\
\hline 2044 & 25.091 & 12.30 & 12.31 & 12.31 & 12.32 & 12.30 & 12.30 & 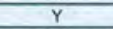 & 6.3397 & pass \\
\hline 2045 & 25.163 & 12.31 & 12.31 & 12.32 & 12.32 & 12.31 & 12.31 & $\begin{array}{l} \\
\end{array}$ & 6.3478 & pass \\
\hline 2046 & 25.076 & 12.30 & 12.30 & 12.32 & 12.32 & 12.30 & 12.30 & $\mathrm{Y}$ & 6.3524 & pass \\
\hline 2047 & 25.205 & 12.31 & 12.32 & 12.32 & 12.32 & 12.32 & 12.32 & $\mathrm{Y}$ & 6.3480 & pass \\
\hline 2048 & 25.120 & 12.32 & 12.31 & 12.32 & 12.32 & 12.31 & 12.32 & $\mathrm{Y}$ & 6.3350 & pass \\
\hline 2049 & 25.245 & 12.31 & 12.31 & 12.32 & 12.32 & 12.30 & 12.31 & $Y$ & 6.3393 & pass \\
\hline 2050 & 25.134 & 12.31 & 12.32 & 12.34 & 12.34 & 12.31 & 12.32 & $\begin{array}{l} \\
\end{array}$ & 6.3517 & pass \\
\hline 2051 & 25.201 & 12.31 & 12.32 & 12.32 & 12.32 & 12.30 & 12.31 & $\begin{array}{l} \\
\end{array}$ & 6.3400 & pass \\
\hline 2052 & 25.234 & 12.31 & 12.32 & 12.32 & 12.33 & 12.32 & 12.32 & $\bar{Y}$ & 6.3528 & pass \\
\hline 2053 & 25.273 & 12.30 & 12.31 & 12.32 & 12.33 & 12.31 & 12.31 & $Y$ & 6.3563 & pass \\
\hline 2054 & 25.157 & 12.32 & 12.32 & 12.33 & 12.33 & 12.32 & 12.32 & $Y$ & 6.3552 & pass \\
\hline 2055 & 25.062 & 12.31 & 12.32 & 12.31 & 12.32 & 12.32 & 12.31 & $Y$ & 6.3535 & pass \\
\hline 2056 & 25.241 & 12.31 & 12.31 & 12.32 & 12.32 & 12.31 & 12.32 & $Y$ & 6.3569 & pass \\
\hline 2057 & 25.242 & 12.31 & 12.30 & 12.31 & 12.31 & 12.30 & 12.31 & $\bar{Y}$ & 6.3532 & pass \\
\hline 2058 & 25.174 & 12.32 & 12.31 & 12.32 & 12.31 & 12.32 & 12.31 & $Y$ & 6.3426 & pass \\
\hline 2059 & 25.030 & 12.30 & 12.30 & 12.31 & 12.31 & 12.31 & 12.30 & $Y$ & 6.3389 & pass \\
\hline 2060 & 25.267 & 12.32 & 12.32 & 12.33 & 12.33 & 12.33 & 12.32 & $Y$ & 6.3550 & pass \\
\hline 2061 & 25.336 & 12.33 & 12.32 & 12.33 & 12.33 & 12.33 & 12.32 & $Y$ & 6.3425 & pass \\
\hline 2062 & 25.218 & 12.31 & 12.32 & 12.32 & 12.32 & 12.30 & 12.31 & $Y$ & 6.3434 & pass \\
\hline 2063 & 25.095 & 12.32 & 12.32 & 12.33 & 12.33 & 12.32 & 12.32 & $Y$ & 6.3432 & pass \\
\hline 2064 & 25.131 & 12.32 & 12.32 & 12.32 & 12.32 & 12.31 & 12.31 & $Y$ & 6.3355 & pass \\
\hline 2065 & 25.288 & 12.32 & 12.32 & 12.33 & 12.33 & 12.32 & 12.32 & $Y$ & 6.3456 & pass \\
\hline 2066 & 25.134 & 12.33 & 12.32 & 12.33 & 12.33 & 12.32 & 12.32 & $Y$ & 6.3521 & pass \\
\hline 2067 & 25.212 & 12.31 & 12.32 & 12.33 & 12.33 & 12.31 & 12.32 & $Y$ & 6.3400 & pass \\
\hline 2068 & 25.165 & 12.31 & 12.31 & 12.32 & 12.32 & 12.32 & 12.32 & $\bar{Y}$ & 6.3575 & pass \\
\hline 2069 & 25.214 & 12.32 & 12.32 & 12.33 & 12.33 & 12.31 & 12.31 & $Y$ & 6.3450 & pass \\
\hline 2070 & 25.177 & 12.31 & 12.31 & 12.33 & 12.33 & 12.31 & 12.31 & $Y$ & 6.3416 & pass \\
\hline 2071 & 25.215 & 12.32 & 12.32 & 12.32 & 12.33 & 12.32 & 12.31 & $Y$ & 6.3573 & pass \\
\hline Z072 & 25.152 & 12.31 & 12.31 & 12.32 & 12.32 & 12.30 & 12.30 & $Y$ & 6.3374 & pass \\
\hline 2073 & 25.246 & 12.32 & 12.32 & 12.33 & 12.33 & 12.32 & 12.32 & $Y$ & 6.3604 & pass \\
\hline 2074 & 25.134 & 12.32 & 12.31 & 12.32 & 12.32 & 12.31 & 12.31 & $Y$ & 6.3449 & pass \\
\hline 2075 & 25.234 & 12.32 & 12.32 & 12.33 & 12.33 & 12.32 & 12.32 & $Y$ & 6.3514 & pass \\
\hline 2076 & 25.251 & 12.31 & 12.31 & 12.32 & 12.32 & 12.31 & 12.31 & $Y$ & 6.3512 & pass \\
\hline 2077 & 25.143 & 12.32 & 12.32 & 12.32 & 12.33 & 12.31 & 12.31 & $Y$ & 6.3576 & pass \\
\hline 2078 & 25.234 & 12.31 & 12.31 & 12.32 & 12.32 & 12.31 & 12.32 & $Y$ & 6.3293 & pass \\
\hline 2079 & 25.315 & 12.31 & 12.30 & 12.32 & 12.32 & 12.32 & 12.32 & $Y$ & 6.3491 & pass \\
\hline 2080 & 25.161 & 12.32 & 12.31 & 12.32 & 12.32 & 12.30 & 12.31 & $Y$ & 6.3478 & pass \\
\hline
\end{tabular}

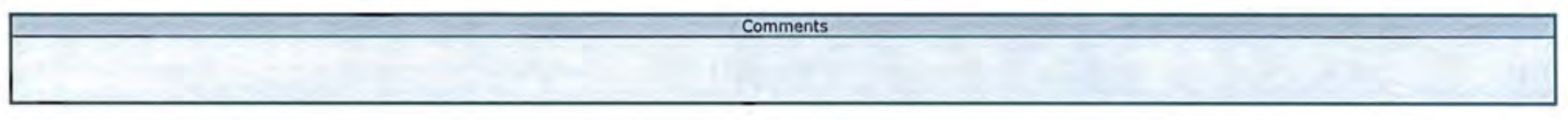

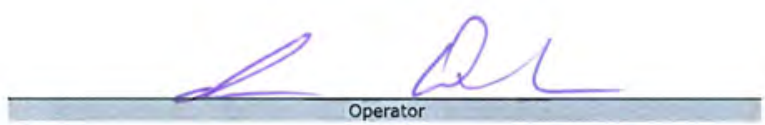

Whin acsupervisor

QA Review

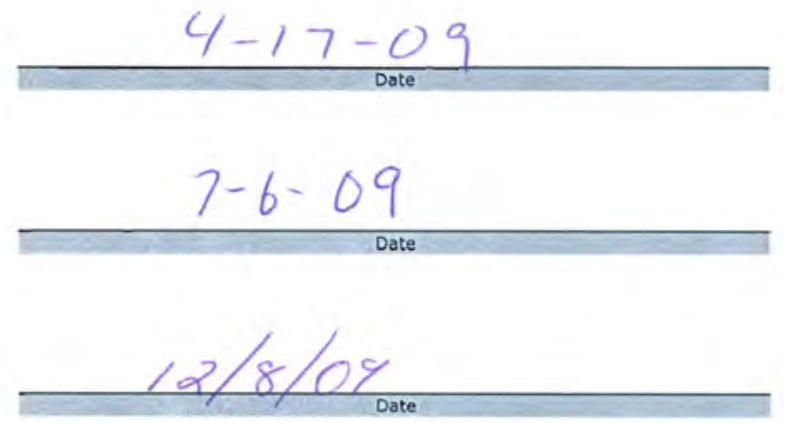


Data Report Form DRF-24A: Compact Diameter and Length

\begin{tabular}{|c|c|}
\hline Procedure: & AGR-CHAR-DAM- 24 Rev. 6 \\
\hline Operator: & Ivan Dunber \\
\hline Compact lot ID: & LEU07-OP1-Z \\
\hline Compact Lot description: & AGR-2 UCO Baseline, from G73]-14-93072A \\
\hline Filename: & IImc-aarVAGR\CompactDimensions\LEU07-OP1_DRF24R6.xls \\
\hline Vertical height gauge calibration due date: & $3 / 6 / 10$ \\
\hline Pass-thru block calibration due date: & $1 / 17 / 11$ \\
\hline Digital caliper calibration due date: & $7 / 22 / 09$ \\
\hline Gauge blocks calibration due date: & $11 / 7 / 12$ \\
\hline Analytical balance callbration due date: & $2 / 12 / 10$ \\
\hline
\end{tabular}

\begin{tabular}{|r|l|}
\hline Acceptance criteria for compact length: & $\geq 25.02$ and $\leq 25.40 \mathrm{~mm}$ \\
\hline Acceptance criteria for compact diameter: & $\geq 12.22$ and $\leq 12.46 \mathrm{~mm}$ (and pass through 12.46 mm ring gauge) \\
\hline Acceptance criteria for compact mass: & For information only \\
\hline
\end{tabular}

\begin{tabular}{|c|c|c|c|c|c|c|c|c|c|c|}
\hline \multirow{2}{*}{$\begin{array}{l}\text { Compact } \\
\text { ID Number }\end{array}$} & \multirow{2}{*}{$\begin{array}{l}\text { Length } \\
(\mathrm{mm})\end{array}$} & \multicolumn{6}{|c|}{ Diameter $(\mathrm{mm})$} & \multirow{2}{*}{$\begin{array}{l}\text { Pass Thru? } \\
(Y \text { or } N)\end{array}$} & \multirow{2}{*}{$\begin{array}{l}\text { Compact weight } \\
\text { (a) }\end{array}$} & \multirow{2}{*}{$\begin{array}{c}\text { Accept? } \\
\text { (pass or fail) }\end{array}$} \\
\hline & & Top 1 & Top 2 & Middle 1 & Middle 2 & Bottom 1 & Bottom 2 & & & \\
\hline 2081 & 25.083 & 12.31 & 12.31 & 12.32 & 12.32 & 12.31 & 12.31 & $\mathrm{Y}$ & 6.3519 & pass \\
\hline 2082 & 25.078 & 12.31 & 12.32 & 12.31 & 12.32 & 12.31 & 12.30 & $Y$ & 6.3452 & pass \\
\hline 2083 & 25.155 & 12.31 & 12.32 & 12.32 & 12.32 & 12.31 & 12.31 & $\bar{Y}$ & 6.3436 & pass \\
\hline 2084 & 25.219 & 12.32 & 12.32 & 12.33 & 12.33 & 12.32 & 12.31 & $\bar{Y}$ & 6.3638 & pass \\
\hline 2085 & 25.196 & 12.32 & 12.30 & 12.33 & 12.32 & 12.31 & 12.31 & $\bar{Y}$ & 6.3373 & pass \\
\hline 2086 & 25.219 & 12.32 & 12.32 & 12.33 & 12.33 & 12.32 & 12.32 & $\bar{Y}$ & 6.3558 & pass \\
\hline 2087 & 25.340 & 12.32 & 12.32 & 12.33 & 12.33 & 12.32 & 12.32 & $\mathrm{Y}$ & 6.3544 & pass \\
\hline 2088 & 25.236 & 12.31 & 12.31 & 12.32 & 12.32 & 12.31 & 12.31 & $\bar{Y}$ & 6.3484 & pass \\
\hline 2089 & 25.223 & 12.32 & 12.32 & 12.33 & 12.33 & 12.32 & 12.32 & $Y$ & 6.3548 & pass \\
\hline Z091 & 25.115 & 12.32 & 12.32 & 12.33 & 12.33 & 12.32 & 12.31 & $Y$ & 6.3576 & pass \\
\hline 2092 & 25.147 & 12.30 & 12.30 & 12.32 & 12.32 & 12.30 & 12.31 & $Y$ & 6.3390 & pass \\
\hline Z093 & 25.163 & 12.31 & 12.31 & 12.32 & 12.32 & 12.31 & 12.31 & $Y$ & 6.3559 & pass \\
\hline Z094 & 25.135 & 12.31 & 12.31 & 12.32 & 12.32 & 12.31 & 12.31 & $\bar{Y}$ & 6.3409 & pass \\
\hline 2095 & 25.233 & 12.31 & 12.31 & 12.33 & 12.33 & 12.32 & 12.32 & $Y$ & 6.3616 & pass \\
\hline Z096 & 25.252 & 12.31 & 12.31 & 12.32 & 12.32 & 12.30 & 12.31 & $Y$ & 6.3530 & pass \\
\hline 2097 & 25.185 & 12.31 & 12.31 & 12.32 & 12.33 & 12.32 & 12.31 & $Y$ & 6.3451 & pass \\
\hline 2098 & 25.195 & 12.32 & 12.31 & 12.33 & 12.33 & 12.32 & 12.32 & $Y$ & 6.3492 & pass \\
\hline Z099 & 25.206 & 12.32 & 12.31 & 12.33 & 12.32 & 12.30 & 12.31 & $Y$ & 6.3549 & pass \\
\hline $\mathrm{Z100}$ & 25.142 & 12.31 & 12.31 & 12.33 & 12.32 & 12.32 & 12.31 & $Y$ & 6.3409 & pass \\
\hline$Z 101$ & 25.146 & 12.30 & 12.30 & 12.32 & 12.32 & 12.30 & 12.31 & $Y$ & 6.3309 & pass \\
\hline Z104 & 25.077 & 12.30 & 12.31 & 12.32 & 12.32 & 12.30 & 12.30 & $Y$ & 6.3266 & pass \\
\hline Z105 & 25.133 & 12.30 & 12.30 & 12.32 & 12.32 & 12.31 & 12.31 & $Y$ & 6.3386 & pass \\
\hline$Z 106$ & 25.184 & 12.30 & 12.31 & 12.32 & 12.33 & 12.30 & 12.31 & $Y$ & 6.3277 & pass \\
\hline$Z 107$ & 25.175 & 12.31 & 12.31 & 12.33 & 12.33 & 12.31 & 12.32 & $Y$ & 6.3457 & pass \\
\hline $\mathrm{Z108}$ & 25.151 & 12.31 & 12.31 & 12.33 & 12.33 & 12.32 & 12.32 & $Y$ & 6.3570 & pass \\
\hline Z109 & 25.194 & 12.32 & 12.32 & 12.34 & 12.33 & 12.32 & 12.31 & $Y$ & 6.3308 & pass \\
\hline$Z 110$ & 25.032 & 12.31 & 12.31 & 12.32 & 12.33 & 12.31 & 12.31 & $Y$ & 6.3430 & pass \\
\hline$Z 111$ & 25.238 & 12.31 & 12.32 & 12.32 & 12.33 & 12.31 & 12.31 & $Y$ & 6.3402 & pass \\
\hline$Z 112$ & 25.217 & 12.30 & 12.30 & 12.32 & 12.32 & 12.30 & 12.31 & $Y$ & 6.3430 & pass \\
\hline $\mathrm{Z113}$ & 25.177 & 12.30 & 12.30 & 12.32 & 12.31 & 12.30 & 12.31 & $Y$ & 6.3466 & pass \\
\hline $\mathrm{Z114}$ & 25.142 & 12.31 & 12.30 & 12.32 & 12.31 & 12.30 & 12.31 & $Y$ & 6.3289 & pass \\
\hline Z115 & 25.192 & 12.31 & 12.32 & 12.32 & 12.32 & 12.31 & 12.31 & $Y$ & 6.3347 & pass \\
\hline$Z 116$ & 25.278 & 12.32 & 12.32 & 12.33 & 12.33 & 12.32 & 12.32 & $Y$ & 6.3665 & pass \\
\hline$Z 117$ & 25.135 & 12.30 & 12.30 & 12.32 & 12.32 & 12.31 & 12.31 & $\bar{Y}$ & 6.3332 & pass \\
\hline $\mathrm{Z} 118$ & 25.228 & 12.30 & 12.31 & 12.32 & 12.32 & 12.31 & 12.31 & $Y$ & 6.3454 & pass \\
\hline Z119 & 25.112 & 12.32 & 12.31 & 12.32 & 12.32 & 12.30 & 12.30 & $\mathrm{Y}$ & 6.3501 & pass \\
\hline $\mathrm{Z} 120$ & 25.298 & 12.32 & 12.31 & 12.32 & 12.32 & 12.30 & 12.31 & $Y$ & 6.3380 & pass \\
\hline
\end{tabular}

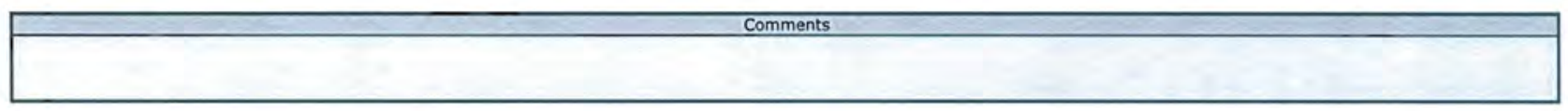

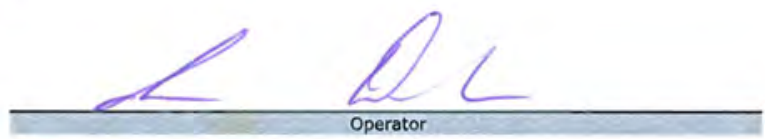
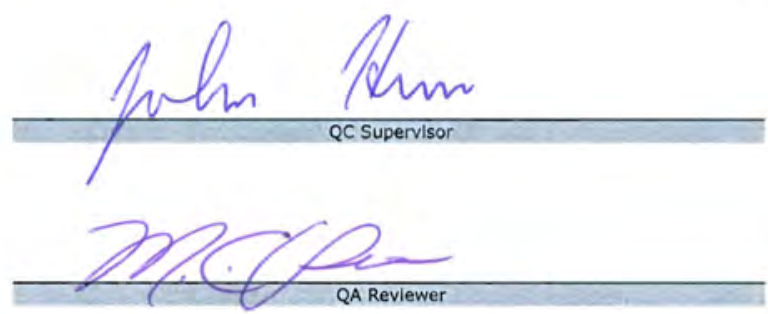

$$
4-17-09
$$$$
7-6-09
$$
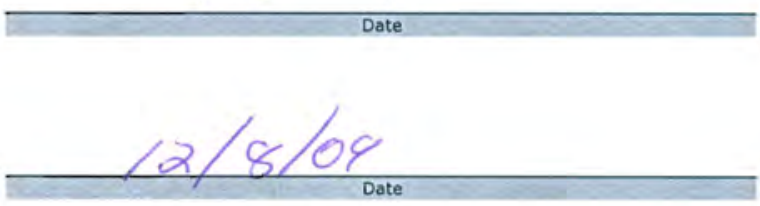
ORNL/TM-2010/296
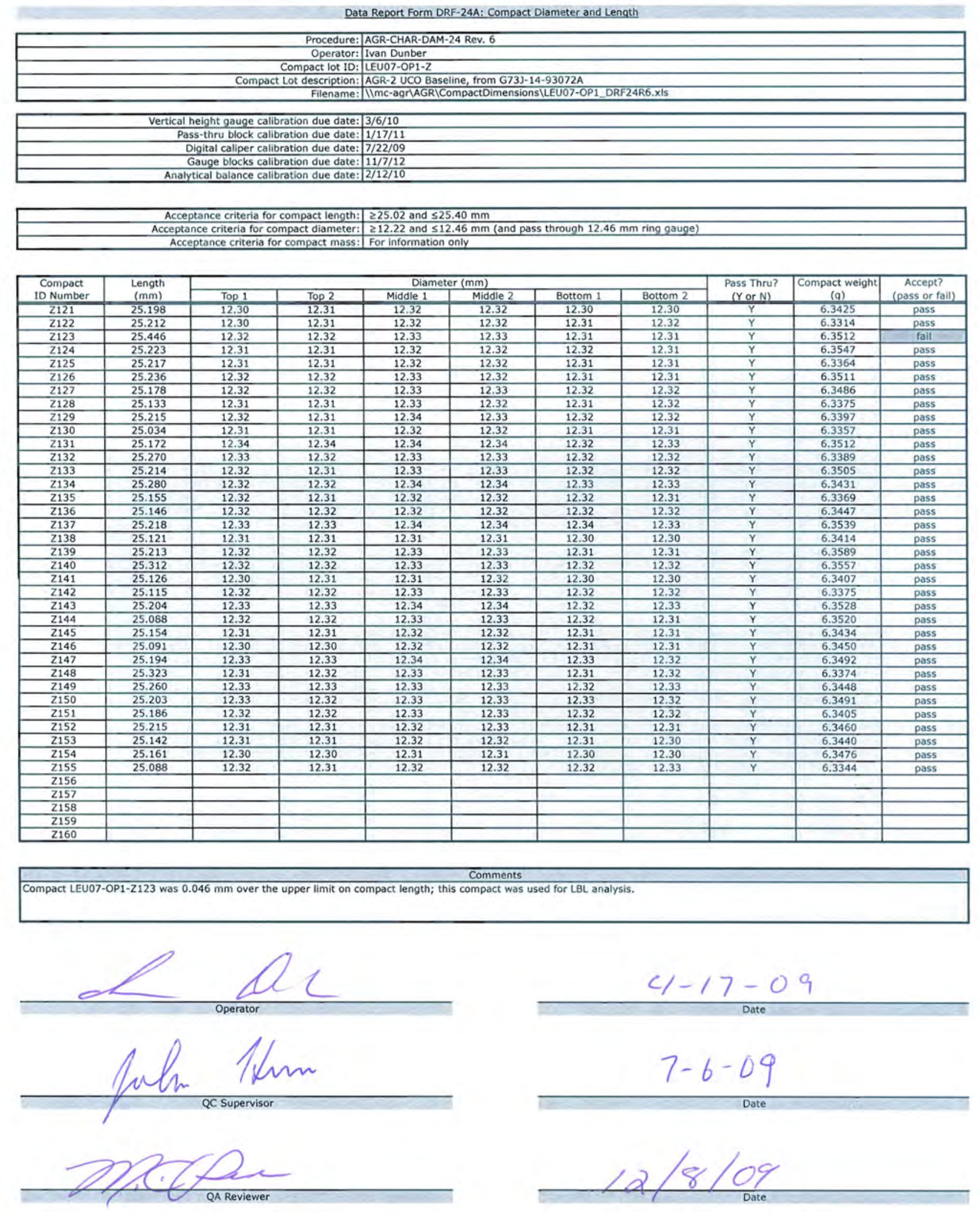

28 
ORNL/TM-2010/296
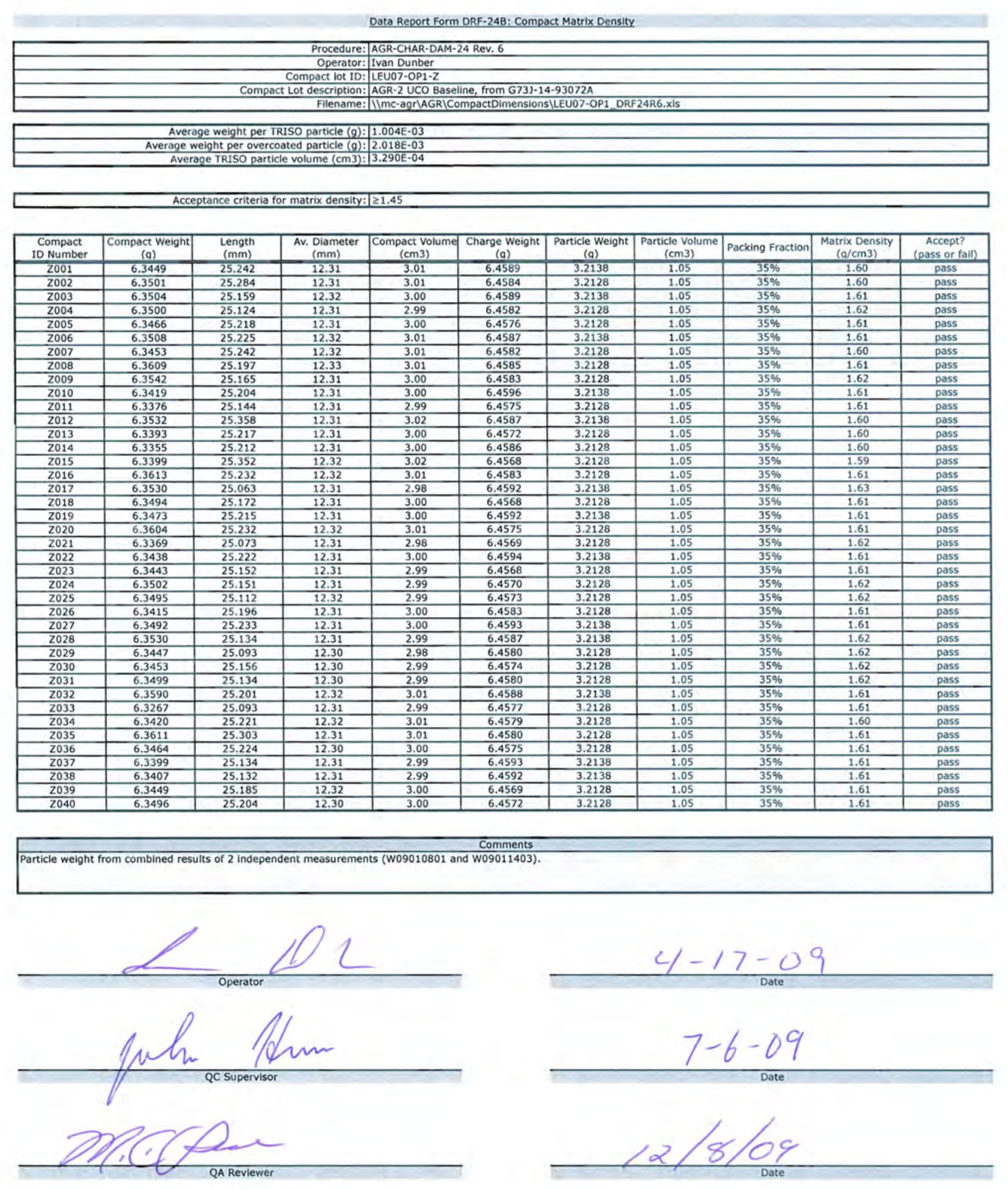

29 
Data Report Form DRF-24B: Compact Matrix Density

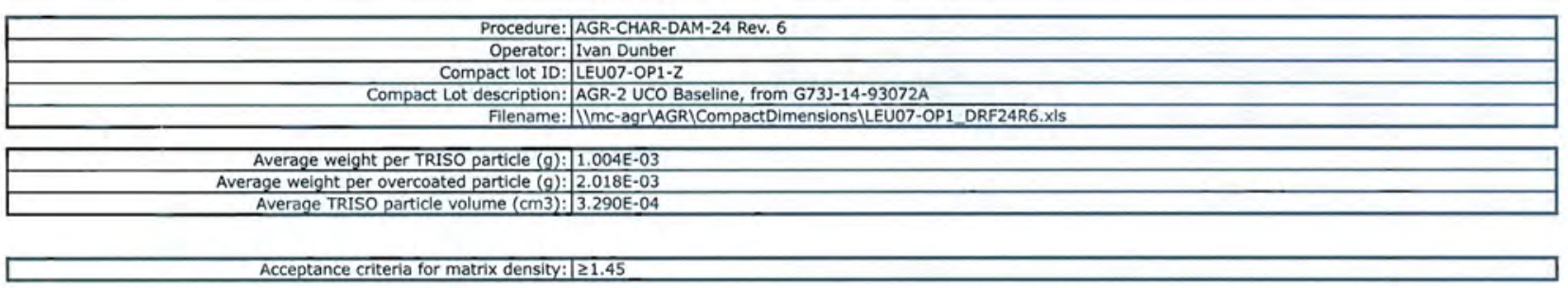

\begin{tabular}{|c|c|c|c|c|c|c|c|c|c|c|}
\hline $\begin{array}{l}\text { Compact } \\
\text { ID Number }\end{array}$ & $\begin{array}{l}\text { Compact Weight } \\
\text { (a) }\end{array}$ & $\begin{array}{l}\text { Length } \\
(\mathrm{mm})\end{array}$ & $\begin{array}{l}\text { Av. Diameter } \\
(\mathrm{mm})\end{array}$ & $\begin{array}{l}\text { Compact Volume } \\
(\mathrm{cm} 3)\end{array}$ & $\begin{array}{l}\text { Charge Weight } \\
\text { (a) }\end{array}$ & $\begin{array}{l}\text { Particle Weight } \\
\text { (a) }\end{array}$ & $\begin{array}{l}\text { Particle Volume } \\
\text { (cm3) }\end{array}$ & Packing Fraction & $\begin{array}{l}\text { Matrix Density } \\
(\mathrm{a} / \mathrm{cm} 3)\end{array}$ & $\begin{array}{c}\text { Accept? } \\
\text { (pass or fail) }\end{array}$ \\
\hline 2041 & \begin{tabular}{|l|}
6.3464 \\
\end{tabular} & 25.121 & 12.31 & $\begin{array}{l}.99 \\
\end{array}$ & 6.4576 & 3.2128 & 1.05 & $35 \%$ & 1.62 & pass \\
\hline 2042 & 6.3584 & 25.226 & 12.32 & 3.01 & 6.4564 & 3.2118 & 1.05 & $35 \%$ & 1.61 & pass \\
\hline 2043 & 6.3437 & 25.063 & 12.31 & 2.98 & 6.4593 & 3.2138 & 1.05 & $35 \%$ & 1.62 & pass \\
\hline 2044 & 6.3397 & 25.091 & 12.31 & 2.98 & 6.4595 & 3.2138 & 1.05 & $35 \%$ & 1.62 & pass \\
\hline 2045 & 6.3478 & 25.163 & 12.31 & 3.00 & 6.4594 & 3.2138 & 1.05 & $35 \%$ & 1.61 & pass \\
\hline 2046 & 6.3524 & 25.076 & 12.31 & 2.98 & 6.4577 & 3.2128 & 1.05 & $35 \%$ & 1.63 & pass \\
\hline 2047 & 6.3480 & 25.205 & 12.32 & 3.00 & 6.4572 & 3.2128 & 1.05 & $35 \%$ & 1.61 & pass \\
\hline 2048 & 6.3350 & 25.120 & 12.32 & 2.99 & 6.4583 & 3.2128 & 1.05 & $35 \%$ & 1.61 & pass \\
\hline 2049 & 6.3393 & 25.245 & 12.31 & 3.01 & 6.4585 & 3.2128 & 1.05 & $35 \%$ & 1.60 & pass \\
\hline 2050 & $\frac{0.359}{6.3517}$ & $\frac{25.145}{25.134}$ & 12.32 & 3.00 & 6.45950 & 3.2138 & 1.05 & $35 \%$ & 1.61 & $\frac{p a s s}{\text { pass }}$ \\
\hline Z051 & 6.3400 & 25.201 & 12.31 & 3.00 & 6.4579 & 3.2128 & 1.05 & $35 \%$ & 1.61 & pass \\
\hline $\mathrm{Z} 052$ & 6.3528 & 25.234 & 12.32 & 3.01 & 6.4574 & 3.2128 & 1.05 & $35 \%$ & 1.61 & pass \\
\hline 2053 & 6.3563 & 25.273 & 12.31 & 3.01 & 6.4597 & 3.2138 & 1.05 & $35 \%$ & 1.61 & pass \\
\hline 2054 & 6.3552 & 25.157 & 12.32 & 3.00 & 6.4580 & 3.2128 & 1.05 & $35 \%$ & 1.61 & pass \\
\hline 2055 & 6.3535 & 25.062 & 12.32 & 2.99 & 6.4581 & 3.2128 & 1.05 & $35 \%$ & 1.63 & pass \\
\hline 2056 & 6.3569 & 25.241 & 12.32 & 3.01 & 6.4589 & 3.2138 & 1.05 & $35 \%$ & 1.61 & pass \\
\hline 2057 & 6.3532 & 25.242 & 12.31 & 3.00 & 6.4575 & 3.2128 & 1.05 & $35 \%$ & 1.61 & pass \\
\hline 2058 & 6.3426 & 25.174 & 12.32 & 3.00 & 6.4591 & 3.2138 & 1.05 & $35 \%$ & 1.61 & pass \\
\hline 2059 & 6.3389 & 25.030 & 12.31 & 2.98 & 6.4583 & 3.2128 & 1.05 & $35 \%$ & 1.62 & pass \\
\hline 2060 & 6.3550 & 25.267 & 12.33 & 3.01 & 6.4583 & 3.2128 & 1.05 & $35 \%$ & 1.60 & pass \\
\hline 2061 & 6.3425 & 25.336 & 12.33 & 3.02 & 6.4581 & 3.2128 & 1.05 & $35 \%$ & 1.59 & pass \\
\hline 2062 & 6.3434 & 25.218 & 12.31 & 3.00 & 6.4575 & 3.2128 & 1.05 & $35 \%$ & 1.61 & pass \\
\hline Z063 & 6.3432 & 25.095 & 12.32 & 2.99 & 6.4593 & 3.2138 & 1.05 & $35 \%$ & 1.61 & pass \\
\hline 2064 & 6.3355 & 25.131 & 12.32 & 2.99 & 6.4579 & 3.2128 & 1.05 & $35 \%$ & 1.61 & pass \\
\hline 2065 & 6.3456 & 25.288 & 12.32 & 3.02 & 6.4586 & 3.2128 & 1.05 & $35 \%$ & 1.60 & pass \\
\hline 2066 & 6.3521 & 25.134 & 12.33 & 3.00 & 6.4591 & 3.2138 & 1.05 & $35 \%$ & 1.61 & pass \\
\hline 2067 & 6.3400 & 25.212 & 12.32 & 3.01 & 6.4585 & 3.2128 & 1.05 & $35 \%$ & 1.60 & pass \\
\hline 2068 & 6.3575 & 25.165 & 12.32 & 3.00 & 6.4587 & 3.2138 & 1.05 & $35 \%$ & 1.62 & pass \\
\hline 2069 & 6.3450 & 25.214 & 12.32 & 3.01 & 6.4582 & 3.2128 & 1.05 & $35 \%$ & 1.60 & pass \\
\hline 2070 & 6.3416 & $\frac{25.147}{25.177}$ & 12.32 & 3.00 & $\frac{0.4502}{6.4572}$ & 3.2128 & 1.05 & $35 \%$ & 1.61 & $\frac{p \text { pass }}{\text { pass }}$ \\
\hline Z071 & 6.3573 & 25.215 & 12.32 & 3.01 & 6.4591 & 3.2138 & 1.05 & $35 \%$ & 1.61 & pass \\
\hline 2072 & 6.3374 & 25.152 & 12.31 & 2.99 & 6.4590 & 3.2138 & 1.05 & $35 \%$ & 1.61 & pass \\
\hline 2073 & 6.3604 & 25.246 & 12.32 & 3.01 & 6.4583 & 3.2128 & 1.05 & $35 \%$ & 1.61 & pass \\
\hline 2074 & 6.3449 & 25.134 & 12.32 & 2.99 & 6.4570 & 3.2128 & 1.05 & $35 \%$ & 1.61 & pass \\
\hline 2075 & 6.3514 & 25.234 & 12.32 & 3.01 & 6.4575 & 3.2128 & 1.05 & $35 \%$ & 1.60 & pass \\
\hline 2076 & 6.3512 & 25.251 & 12.31 & 3.01 & 6.4 & 3.2 & 1.05 & $35 \%$ & 1.61 & pass \\
\hline 2077 & 6.3576 & 25.143 & 12.32 & 3.00 & 6.4595 & 3.2138 & 1.05 & $35 \%$ & 1.62 & pass \\
\hline 2078 & 6.3293 & 25.234 & 12.32 & 3.01 & 6.4592 & 3.2138 & 1.05 & $35 \%$ & 1.60 & pass \\
\hline 2079 & 6.3491 & 25.315 & 12.32 & 3.02 & 6.4587 & 3.2138 & 1.05 & $35 \%$ & 1.60 & pass \\
\hline 2080 & 6.3478 & 25.161 & 12.31 & 3.00 & 6.4597 & 3.2138 & 1.05 & $35 \%$ & 1.61 & pass \\
\hline
\end{tabular}

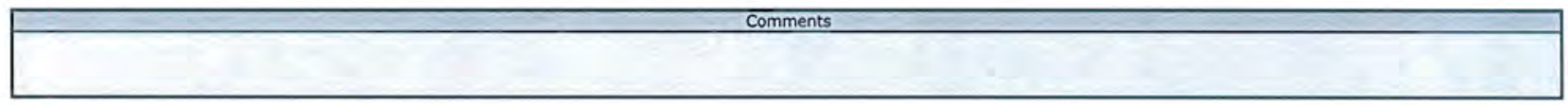

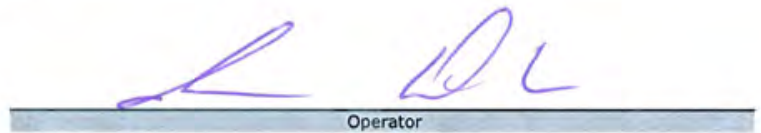
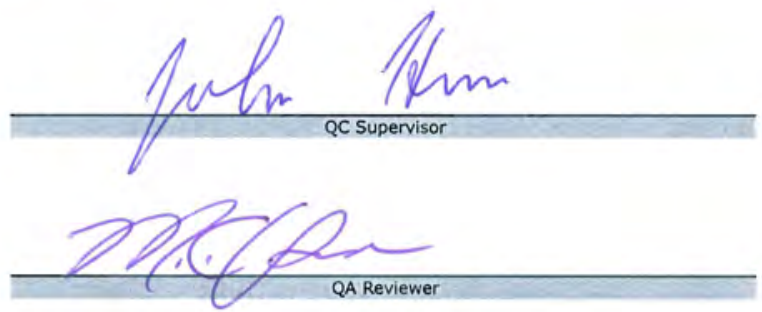

$$
4-17-09
$$

$$
7-6-09
$$
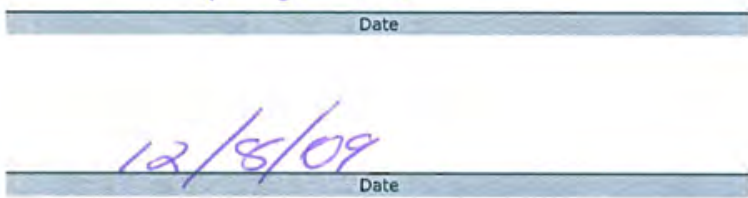
Data Report Form DRF-24B: Compact Matrix Density

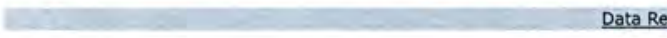

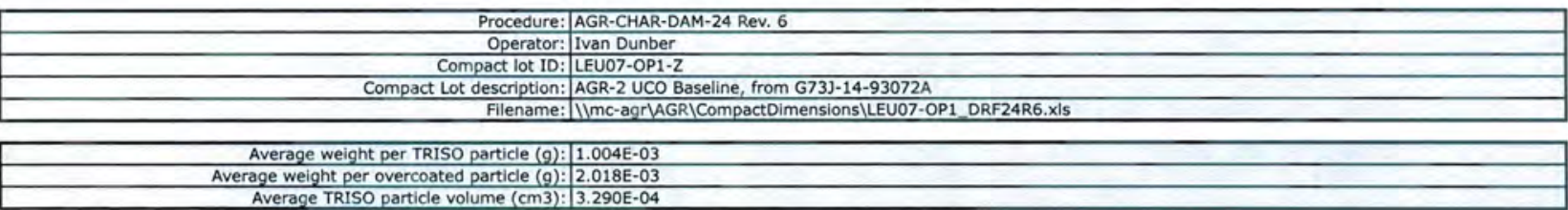
Acceptance criteria for matrix density: $\geq 1.45$

\begin{tabular}{|c|c|c|c|c|c|c|c|c|c|c|}
\hline $\begin{array}{l}\text { Compact } \\
\text { ID Number }\end{array}$ & $\begin{array}{l}\text { Compact Weight } \\
\text { (a) }\end{array}$ & $\begin{array}{l}\text { Length } \\
(\mathrm{mm})\end{array}$ & $\begin{array}{l}\text { Av. Diameter } \\
(\mathrm{mm})\end{array}$ & $\begin{array}{c}\begin{array}{c}\text { Compact Volume } \\
(\mathrm{cm} 3)\end{array} \\
\end{array}$ & $\begin{array}{l}\text { Charge Weight } \\
\text { (a) }\end{array}$ & $\begin{array}{l}\text { Particle Weight } \\
\text { (a) }\end{array}$ & $\begin{array}{c}\text { Particle Volume } \\
(\mathrm{cm} 3)\end{array}$ & Packing Fraction & $\begin{array}{l}\text { Matrix Density } \\
(\mathrm{a} / \mathrm{cm} 3)\end{array}$ & $\begin{array}{c}\text { Accept? } \\
\text { (pass or fail) }\end{array}$ \\
\hline Z081 & 6.3519 & 25.083 & 12.31 & 2.99 & 6.4591 & 3.2138 & 1.05 & $35 \%$ & 1.62 & pass \\
\hline 2082 & 6.3452 & 25.078 & 12.31 & 2.99 & 6.4583 & 3.2128 & 1.05 & $35 \%$ & 1.62 & pass \\
\hline 2083 & 6.3436 & 25.155 & 12.32 & 3.00 & 6.4588 & 3.2138 & 1.05 & $35 \%$ & 1.61 & pass \\
\hline Z084 & 6.3638 & 25.219 & 12.32 & 3.01 & 6.4586 & 3.2128 & 1.05 & $35 \%$ & 1.61 & pass \\
\hline 2085 & 6.3373 & 25.196 & 12.32 & 3.00 & 6.4583 & 3.2128 & 1.05 & $35 \%$ & 1.60 & pass \\
\hline 2086 & 6.3558 & 25.219 & 12.32 & 3.01 & 6.4568 & 3.2128 & 1.05 & $35 \%$ & 1.61 & pass \\
\hline 2087 & 6.3544 & 25.340 & 12.32 & 3.02 & 6.4593 & 3.2138 & 1.05 & $35 \%$ & 1.59 & pass \\
\hline 2088 & 6.3484 & 25.236 & 12.31 & 3.01 & 6.4585 & 3.2128 & 1.05 & $35 \%$ & 1.61 & pass \\
\hline Z089 & 6.3548 & 25.223 & 12.32 & 3.01 & 6.4599 & 3.2138 & 1.05 & $35 \%$ & 1.61 & pass \\
\hline 2090 & 6.3432 & 25.181 & 12.31 & 3.00 & 6.4574 & 3.2128 & 1.05 & $35 \%$ & 1.61 & pass \\
\hline 2091 & 6.3576 & 25.115 & 12.32 & 2.99 & 6.4570 & 3.2128 & 1.05 & $35 \%$ & 1.62 & pass \\
\hline 2092 & 6.3390 & 25.147 & 12.31 & 2.99 & 6.4579 & 3.2128 & 1.05 & $35 \%$ & 1.61 & pass \\
\hline 2093 & 6.3559 & 25.163 & 12.31 & 3.00 & 6.4586 & 3.2128 & 1.05 & $35 \%$ & 1.62 & pass \\
\hline 2094 & 6.3409 & 25.135 & 12.31 & 2.99 & 6.4578 & 3.2128 & 1.05 & $35 \%$ & 1.61 & pass \\
\hline 2095 & 6.3616 & 25.233 & 12.32 & 3.01 & 6.4593 & 3.2138 & 1.05 & $35 \%$ & 1.61 & pass \\
\hline Z096 & 6.3530 & 25.252 & 12.31 & 3.01 & 6.4592 & 3.2138 & 1.05 & $35 \%$ & 1.61 & pass \\
\hline 2097 & 6.3451 & 25.185 & 12.32 & 3.00 & 6.4579 & 3.2128 & 1.05 & $35 \%$ & 1.61 & pass \\
\hline ZO98 & 6.3492 & 25.195 & 12.32 & 3.00 & 6.4577 & 3.2128 & 1.05 & $35 \%$ & 1.61 & pass \\
\hline 2099 & 6.3549 & 25.206 & 12.32 & 3.00 & 6.4586 & 3.2128 & 1.05 & $35 \%$ & 1.61 & pass \\
\hline $\mathrm{Z} 100$ & 6.3409 & 25.142 & 12.32 & 3.00 & 6.4587 & 3.2138 & 1.05 & $35 \%$ & 1.61 & pass \\
\hline $\mathrm{Z} 101$ & 6.3309 & 25.146 & 12.31 & 2.99 & 6.4582 & 3.2128 & 1.05 & $35 \%$ & 1.61 & pass \\
\hline $\mathrm{Z} 102$ & 6.3570 & 25.336 & 12.32 & 3.02 & 6.4582 & 3.2128 & 1.05 & $35 \%$ & 1.60 & pass \\
\hline $\mathrm{Z} 103$ & 6.3386 & 25.195 & 12.33 & 3.01 & 6.4579 & 3.2128 & 1.05 & $35 \%$ & 1.60 & pass \\
\hline $\mathrm{Z104}$ & 6.3266 & 25.077 & 12.31 & 2.98 & 6.4585 & 3.2128 & 1.05 & $35 \%$ & 1.61 & pass \\
\hline $\mathrm{Z} 105$ & 6.3386 & 25.133 & 12.31 & 2.99 & 6.4593 & 3.2138 & 1.05 & $35 \%$ & 1.61 & pass \\
\hline $\mathrm{Z106}$ & 6.3277 & 25.184 & 12.31 & 3.00 & 6.4590 & 3.2138 & 1.05 & $35 \%$ & 1.60 & pass \\
\hline$Z 107$ & 6.3457 & 25.175 & 12.32 & 3.00 & 6.4576 & 3.2128 & 1.05 & $35 \%$ & 1.61 & pass \\
\hline $\mathrm{Z108}$ & 6.3570 & 25.151 & 12.32 & 3.00 & 6.4581 & 3.2128 & 1.05 & $35 \%$ & 1.62 & pass \\
\hline Z109 & 6.3308 & 25.194 & 12.32 & 3.00 & 6.4574 & 3.2128 & 1.05 & $35 \%$ & 1.60 & pass \\
\hline$Z 110$ & 6.3430 & 25.032 & 12.32 & 2.98 & 6.4582 & 3.2128 & 1.05 & $35 \%$ & 1.62 & pass \\
\hline Z111 & 6.3402 & 25.238 & 12.32 & 3.01 & 6.4587 & 3.2138 & 1.05 & $35 \%$ & 1.60 & pass \\
\hline $\mathrm{Z} 112$ & 6.3430 & 25.217 & 12.31 & 3.00 & 6.4578 & 3.2128 & 1.05 & $35 \%$ & 1.61 & pass \\
\hline $\mathrm{Z} 113$ & 6.3466 & 25.177 & 12.31 & 2.99 & 6.4580 & 3.2128 & 1.05 & $35 \%$ & 1.61 & pass \\
\hline 2114 & 6.3289 & 25.142 & 12.31 & 2.99 & 6.4589 & 3.2138 & 1.05 & $35 \%$ & 1.61 & pass \\
\hline Z115 & 6.3347 & 25.192 & 12.32 & 3.00 & 6.4571 & 3.2128 & 1.05 & $35 \%$ & 1.60 & pass \\
\hline$Z 116$ & 6.3665 & 25.278 & 12.32 & 3.02 & 6.4580 & 3.2128 & 1.05 & $35 \%$ & 1.61 & pass \\
\hline $\mathrm{Z} 117$ & 6.3332 & 25.135 & 12.31 & 2.99 & 6.4581 & 3.2128 & 1.05 & $35 \%$ & 1.61 & pass \\
\hline$Z 118$ & 6.3454 & 25.228 & 12.31 & 3.00 & 6.4575 & 3.2128 & 1.05 & $35 \%$ & 1.61 & pass \\
\hline 2119 & 6.3501 & 25.112 & 12.31 & 2.99 & 6.4579 & 3.2128 & 1.05 & $35 \%$ & 1.62 & pass \\
\hline $\mathrm{Z} 120$ & 6.3380 & 25.298 & 12.31 & 3.01 & 6.4582 & 3.2128 & 1.05 & $35 \%$ & 1.59 & pass \\
\hline
\end{tabular}

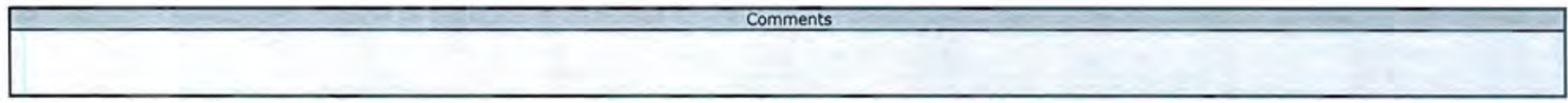

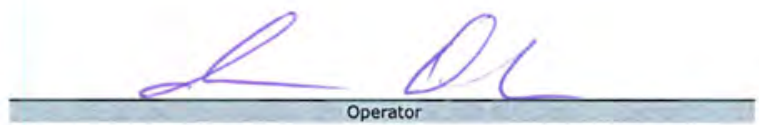
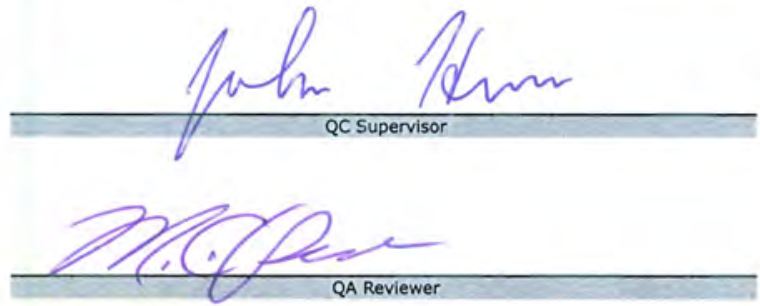

$4-17-09$

$$
7-6-09
$$

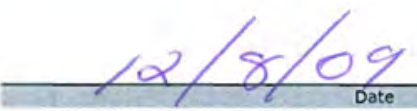




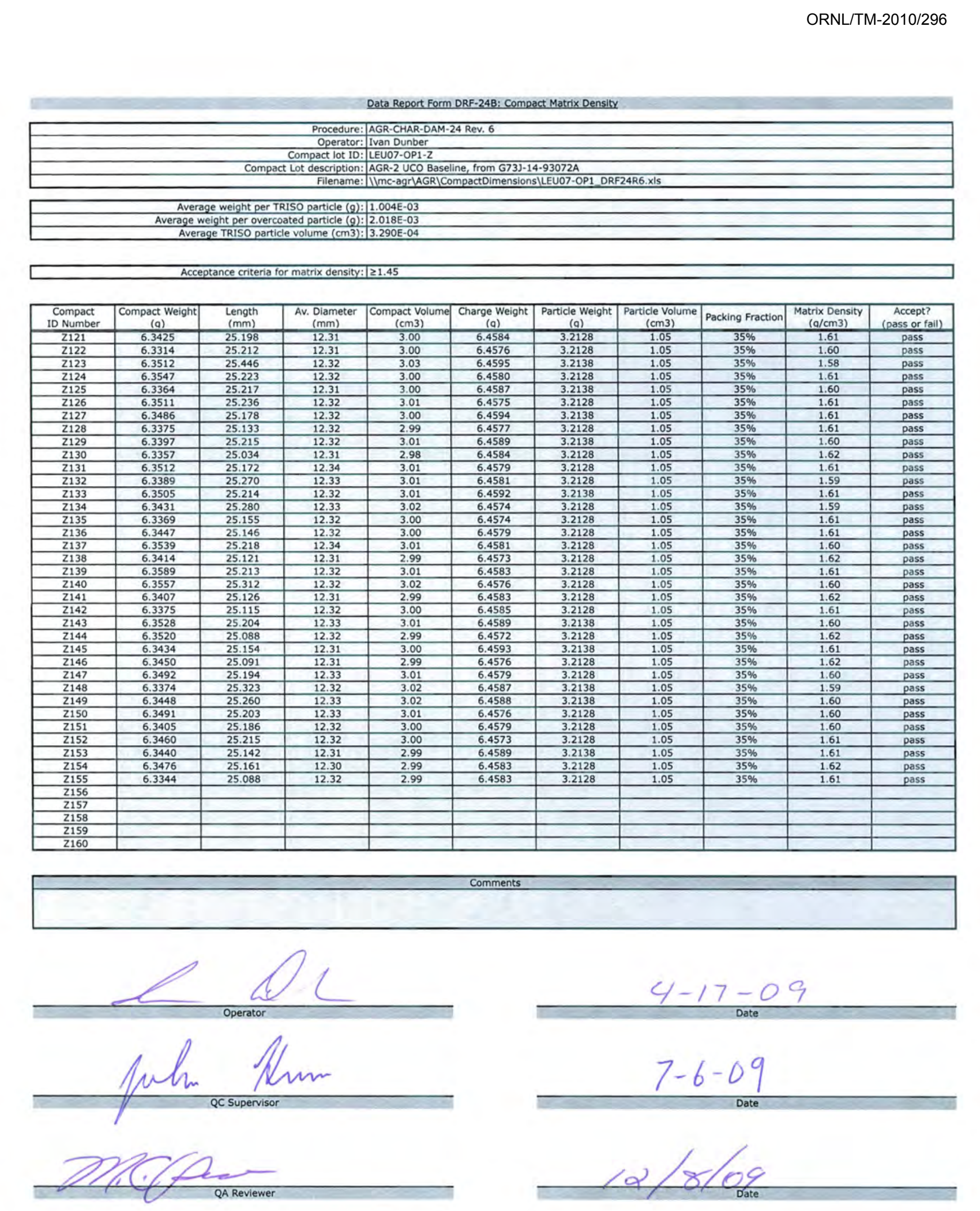


Data Report Form DRF-24C: Compact Tracking

\begin{tabular}{|r|l|}
\hline Procedure: & AGR-CHAR-DAM-24 Rev. 6 \\
\hline Operator: & Ivan Dunber \\
\hline Compact lot ID: & LEU07-OP1-Z \\
\hline Compact Lot description: & AGR-2 UCO Baseline, from G73J-14-93072A \\
\hline Filename: & \ImC-agr\AGR\CompactDimensions\LEU07-OP1_DRF24R6.xIs \\
\hline
\end{tabular}

\begin{tabular}{|c|c|}
\hline $\begin{array}{c}\text { Compact } \\
\text { Z Number }\end{array}$ & $\begin{array}{c}\text { Compact G } \\
\text { Number }\end{array}$ \\
\hline Z001 & G056 \\
\hline Z002 & G049 \\
\hline Z003 & G147 \\
\hline Z004 & G156 \\
\hline Z005 & G132 \\
\hline Z006 & G087 \\
\hline Z007 & G136 \\
\hline Z008 & G152 \\
\hline Z009 & G048 \\
\hline Z010 & G118 \\
\hline Z011 & G010 \\
\hline Z012 & G054 \\
\hline Z013 & G119 \\
\hline Z014 & G021 \\
\hline Z015 & G012 \\
\hline Z016 & G047 \\
\hline Z017 & G107 \\
\hline Z018 & G170 \\
\hline Z019 & G043 \\
\hline Z020 & G130 \\
\hline Z021 & G164 \\
\hline Z022 & G091 \\
\hline Z023 & G161 \\
\hline Z024 & G064 \\
\hline Z025 & G085 \\
\hline Z026 & G101 \\
\hline Z027 & G150 \\
\hline Z028 & G109 \\
\hline Z029 & G040 \\
\hline Z030 & G105 \\
\hline Z031 & G097 \\
\hline Z032 & G090 \\
\hline Z033 & G026 \\
\hline Z034 & G066 \\
\hline Z035 & G044 \\
\hline Z036 & G163 \\
\hline Z037 & G059 \\
\hline Z038 & G079 \\
\hline Z039 & G067 \\
\hline Z040 & G179 \\
\hline
\end{tabular}

\begin{tabular}{|c|c|}
\hline $\begin{array}{c}\text { Compact } \\
\text { Z Number }\end{array}$ & $\begin{array}{c}\text { Compact G } \\
\text { Number }\end{array}$ \\
\hline \hline Z041 & G102 \\
\hline Z042 & G157 \\
\hline Z043 & G100 \\
\hline Z044 & G008 \\
\hline Z045 & G074 \\
\hline Z046 & G103 \\
\hline Z047 & G138 \\
\hline Z048 & G072 \\
\hline Z049 & G020 \\
\hline Z050 & G149 \\
\hline Z051 & G032 \\
\hline Z052 & G165 \\
\hline Z053 & G131 \\
\hline Z054 & G083 \\
\hline Z055 & G098 \\
\hline Z056 & G045 \\
\hline Z057 & G177 \\
\hline Z058 & G146 \\
\hline Z059 & G029 \\
\hline Z060 & G082 \\
\hline Z061 & G057 \\
\hline Z062 & G060 \\
\hline Z063 & G080 \\
\hline Z064 & G073 \\
\hline Z065 & G038 \\
\hline Z066 & G076 \\
\hline Z067 & G144 \\
\hline Z068 & G110 \\
\hline Z069 & G070 \\
\hline Z070 & G078 \\
\hline Z071 & G086 \\
\hline Z072 & G116 \\
\hline Z073 & G111 \\
\hline Z074 & G051 \\
\hline Z075 & G180 \\
\hline Z076 & G151 \\
\hline Z077 & G046 \\
\hline Z078 & G031 \\
\hline Z079 & G127 \\
\hline Z080 & G171 \\
\hline & \\
\hline & \\
\hline
\end{tabular}

\begin{tabular}{|c|c|}
\hline $\begin{array}{c}\text { Compact } \\
\text { Z Number }\end{array}$ & $\begin{array}{c}\text { Compact G } \\
\text { Number }\end{array}$ \\
\hline Z081 & G104 \\
\hline Z082 & G094 \\
\hline Z083 & G142 \\
\hline Z084 & G167 \\
\hline Z085 & G115 \\
\hline Z086 & G129 \\
\hline Z087 & G068 \\
\hline Z088 & G137 \\
\hline Z089 & G140 \\
\hline Z090 & G134 \\
\hline Z091 & G112 \\
\hline Z092 & G124 \\
\hline Z093 & G106 \\
\hline Z094 & G113 \\
\hline Z095 & G172 \\
\hline Z096 & G153 \\
\hline Z097 & G071 \\
\hline Z098 & G162 \\
\hline Z099 & G108 \\
\hline Z100 & G077 \\
\hline Z101 & G033 \\
\hline Z102 & G052 \\
\hline Z103 & G154 \\
\hline Z104 & G023 \\
\hline Z105 & G037 \\
\hline Z106 & G019 \\
\hline Z107 & G143 \\
\hline Z108 & G159 \\
\hline Z109 & G025 \\
\hline Z110 & G093 \\
\hline Z111 & G022 \\
\hline Z112 & G009 \\
\hline Z113 & G062 \\
\hline Z114 & G121 \\
\hline Z115 & G141 \\
\hline Z116 & G174 \\
\hline Z117 & G122 \\
\hline Z118 & G158 \\
\hline Z119 & G095 \\
\hline Z120 & G024 \\
\hline
\end{tabular}

\begin{tabular}{|c|c|}
\hline $\begin{array}{c}\text { Compact } \\
\text { Zumber }\end{array}$ & $\begin{array}{c}\text { Compact G } \\
\text { Number }\end{array}$ \\
\hline Z121 & G042 \\
\hline Z122 & G035 \\
\hline Z123 & G041 \\
\hline Z124 & G173 \\
\hline Z125 & G034 \\
\hline Z126 & G039 \\
\hline Z127 & G148 \\
\hline Z128 & G123 \\
\hline Z129 & G028 \\
\hline Z130 & G013 \\
\hline Z131 & G084 \\
\hline Z132 & G099 \\
\hline Z133 & G061 \\
\hline Z134 & G128 \\
\hline Z135 & G120 \\
\hline Z136 & G178 \\
\hline Z137 & G135 \\
\hline Z138 & G063 \\
\hline Z139 & G175 \\
\hline Z140 & G053 \\
\hline Z141 & G117 \\
\hline Z142 & G155 \\
\hline Z143 & G145 \\
\hline Z144 & G092 \\
\hline Z145 & G069 \\
\hline Z146 & G050 \\
\hline Z147 & G075 \\
\hline Z148 & G125 \\
\hline Z149 & G058 \\
\hline Z150 & G176 \\
\hline Z151 & G114 \\
\hline Z152 & G166 \\
\hline Z153 & G081 \\
\hline Z154 & G160 \\
\hline Z155 & G036 \\
\hline Z156 & \\
\hline Z157 & \\
\hline$Z 158$ & \\
\hline Z159 & \\
\hline
\end{tabular}

Comments

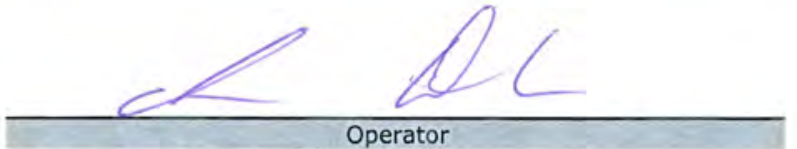

$$
4-15-09
$$


Data Report Form DRF-24D: Compact Charge Weight

\begin{tabular}{|c|c|}
\hline Procedure: & AGR-CHAR-DAM-24 Rev. 6 \\
\hline Operator: & Ivan Dunbar \\
\hline Compact Lot description: & AGR-2 UCO Baseline, from G73J-14-93072A \\
\hline \multicolumn{2}{|c|}{\begin{tabular}{l|l} 
Analytical balance calibration due date: & $10 / 29 / 09$ \\
\end{tabular}} \\
\hline Target compact charge weight $(\mathrm{g})$ : & 6.4580 \\
\hline Average weight per overcoated particle $(\mathrm{g})$ : & $2.018 \mathrm{E}-03$ \\
\hline Approximate number of particles per compact: & 3200 \\
\hline Average uranium loading per particle $(\mathrm{g})$ : & $3.930 \mathrm{E}-04$ \\
\hline Approximate uranium loading per compact $(\mathrm{g})$ : & 1.258 \\
\hline
\end{tabular}

\begin{tabular}{|c|c|}
\hline $\begin{array}{l}\text { Compact } \\
\text { G Number }\end{array}$ & $\begin{array}{c}\text { Charge Weight } \\
\text { (g) }\end{array}$ \\
\hline G001 & 6.4587 \\
\hline $\mathrm{G} 002$ & 6.4581 \\
\hline G003 & 6.4573 \\
\hline G004 & 6.4591 \\
\hline G005 & 6.4570 \\
\hline G006 & 6.4587 \\
\hline G007 & 6.4567 \\
\hline G008 & 6.4595 \\
\hline G009 & 6.4578 \\
\hline G010 & 6.4575 \\
\hline G011 & 6.4580 \\
\hline G012 & 6.4568 \\
\hline G013 & 6.4584 \\
\hline G014 & 6.4584 \\
\hline G015 & 6.4583 \\
\hline G016 & 6.4582 \\
\hline $\mathrm{G} 017$ & 6.4585 \\
\hline G018 & 6.4582 \\
\hline G019 & 6.4590 \\
\hline G020 & 6.4585 \\
\hline G021 & 6.4586 \\
\hline G022 & 6.4587 \\
\hline G023 & 6.4585 \\
\hline G024 & 6.4582 \\
\hline G025 & 6.4574 \\
\hline G026 & 6.4577 \\
\hline $\mathrm{G} 027$ & 6.4580 \\
\hline G028 & 6.4589 \\
\hline G029 & 6.4583 \\
\hline G030 & 6.4579 \\
\hline G031 & 6.4592 \\
\hline G032 & 6.4579 \\
\hline G033 & 6.4582 \\
\hline G034 & 6.4587 \\
\hline G035 & 6.4576 \\
\hline G036 & 6.4583 \\
\hline G037 & 6.4593 \\
\hline G038 & 6.4586 \\
\hline G039 & 6.4575 \\
\hline $\mathrm{G} 040$ & 6.4580 \\
\hline G041 & 6.4595 \\
\hline G042 & 6.4584 \\
\hline G043 & 6.4592 \\
\hline G044 & 6.4580 \\
\hline G045 & 6.4589 \\
\hline
\end{tabular}

\begin{tabular}{|c|c|}
\hline $\begin{array}{c}\text { Compact } \\
\text { G Number }\end{array}$ & $\begin{array}{c}\text { Charge Weight } \\
(\mathrm{g})\end{array}$ \\
\hline G046 & 6.4595 \\
\hline G047 & 6.4583 \\
\hline G048 & 6.4583 \\
\hline G049 & 6.4584 \\
\hline G050 & 6.4576 \\
\hline G051 & 6.4570 \\
\hline G052 & 6.4582 \\
\hline G053 & 6.4576 \\
\hline G054 & 6.4587 \\
\hline G055 & 6.4594 \\
\hline G056 & 6.4589 \\
\hline G057 & 6.4581 \\
\hline G058 & 6.4588 \\
\hline G059 & 6.4593 \\
\hline G060 & 6.4575 \\
\hline G061 & 6.4592 \\
\hline G062 & 6.4580 \\
\hline G063 & 6.4573 \\
\hline G064 & 6.4570 \\
\hline G065 & 6.4572 \\
\hline G066 & 6.4579 \\
\hline G067 & 6.4569 \\
\hline G068 & 6.4593 \\
\hline G069 & 6.4593 \\
\hline G070 & 6.4582 \\
\hline G071 & 6.4579 \\
\hline G072 & 6.4583 \\
\hline G073 & 6.4579 \\
\hline G074 & 6.4594 \\
\hline G075 & 6.4579 \\
\hline G076 & 6.4591 \\
\hline G077 & 6.4587 \\
\hline G078 & 6.4572 \\
\hline G079 & 6.4592 \\
\hline G080 & 6.4593 \\
\hline G081 & 6.4589 \\
\hline G083 & 6.4583 \\
\hline G0856 087 & 6.4580 \\
\hline & 6.4579 \\
\hline G090 & 6.4573 \\
\hline & 6.4591 \\
\hline & 6.4587 \\
\hline
\end{tabular}

\begin{tabular}{|c|c|}
\hline $\begin{array}{c}\text { Compact } \\
\text { G Number }\end{array}$ & $\begin{array}{c}\text { Charge Weight } \\
(\mathrm{g})\end{array}$ \\
\hline G091 & 6.4594 \\
\hline G092 & 6.4572 \\
\hline G093 & 6.4582 \\
\hline G094 & 6.4583 \\
\hline G095 & 6.4579 \\
\hline G096 & 6.4585 \\
\hline G097 & 6.4580 \\
\hline G098 & 6.4581 \\
\hline G099 & 6.4581 \\
\hline G100 & 6.4593 \\
\hline G101 & 6.4583 \\
\hline G102 & 6.4576 \\
\hline G103 & 6.4577 \\
\hline G104 & 6.4591 \\
\hline G105 & 6.4574 \\
\hline G106 & 6.4586 \\
\hline G107 & 6.4592 \\
\hline G108 & 6.4586 \\
\hline G109 & 6.4587 \\
\hline G110 & 6.4587 \\
\hline G111 & 6.4583 \\
\hline G112 & 6.4570 \\
\hline G113 & 6.4578 \\
\hline G114 & 6.4579 \\
\hline G115 & 6.4583 \\
\hline G116 & 6.4590 \\
\hline G117 & 6.4583 \\
\hline G118 & 6.4596 \\
\hline G119 & 6.4572 \\
\hline G120 & 6.4574 \\
\hline G121 & 6.4589 \\
\hline G122 & 6.4581 \\
\hline G123 & 6.4577 \\
\hline G124 & 6.4579 \\
\hline G125 & 6.4587 \\
\hline G126 & 6.4582 \\
\hline G128 & 6.4587 \\
\hline G129 & 6.45730 \\
\hline G131 & 6.4568 \\
\hline & 6.4575 \\
\hline G135 & 6.4577 \\
\hline & 6.4577 \\
\hline & 6.4581 \\
\hline
\end{tabular}

\begin{tabular}{|c|c|}
\hline $\begin{array}{c}\text { Compact } \\
\text { G Number }\end{array}$ & $\begin{array}{c}\text { Charge Weight } \\
(\text { ( ) }\end{array}$ \\
\hline G136 & 6.4582 \\
\hline G137 & 6.4585 \\
\hline G138 & 6.4572 \\
\hline G139 & 6.4561 \\
\hline G140 & 6.4599 \\
\hline G141 & 6.4571 \\
\hline G142 & 6.4588 \\
\hline G143 & 6.4576 \\
\hline G144 & 6.4585 \\
\hline G145 & 6.4589 \\
\hline G146 & 6.4591 \\
\hline G147 & 6.4589 \\
\hline G148 & 6.4594 \\
\hline G149 & 6.4590 \\
\hline G150 & 6.4593 \\
\hline G151 & 6.4581 \\
\hline G152 & 6.4585 \\
\hline G153 & 6.4592 \\
\hline G154 & 6.4579 \\
\hline G155 & 6.4585 \\
\hline G156 & 6.4582 \\
\hline G157 & 6.4564 \\
\hline G158 & 6.4575 \\
\hline G159 & 6.4581 \\
\hline G160 & 6.4583 \\
\hline G161 & 6.4568 \\
\hline G162 & 6.4577 \\
\hline G163 & 6.4575 \\
\hline G164 & 6.4569 \\
\hline G165 & 6.4574 \\
\hline G166 & 6.4573 \\
\hline G167 & 6.4586 \\
\hline G168 & 6.4577 \\
\hline G169 & 6.4584 \\
\hline G170 & 6.4568 \\
\hline G171 & 6.4597 \\
\hline G173 & 6.4593 \\
\hline G174 & 6.4580 \\
\hline G176 & 6.4580 \\
\hline & 6.4583 \\
\hline & 6.4576 \\
\hline G177 & 6.4575 \\
\hline G.4579 \\
\hline G.4572 \\
\hline
\end{tabular}

Comments

$$
4-1-09
$$


ORNL/TM-2010/296
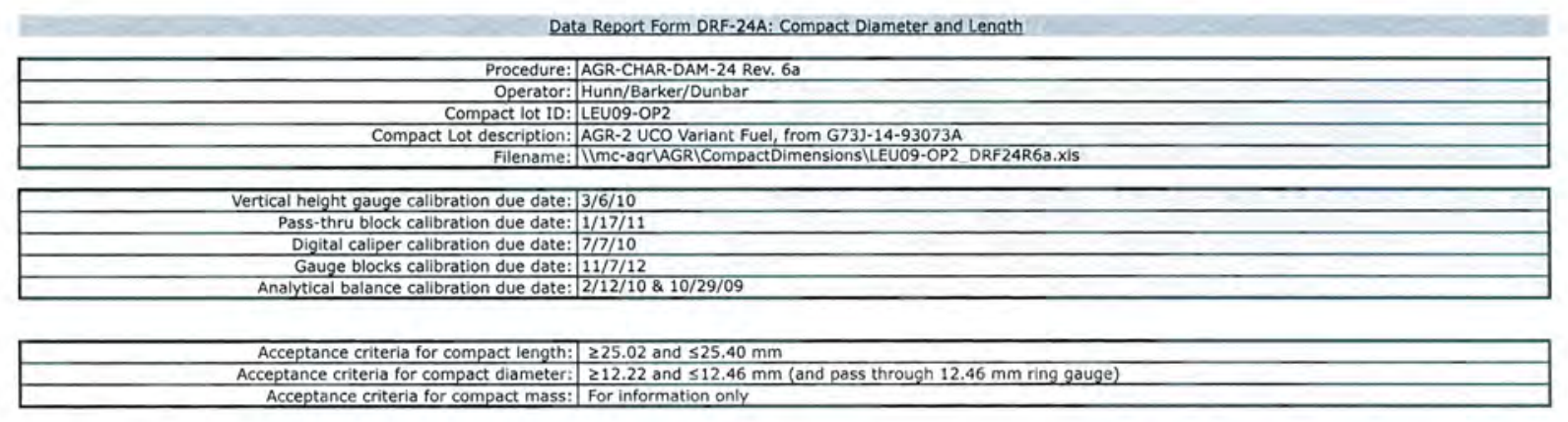

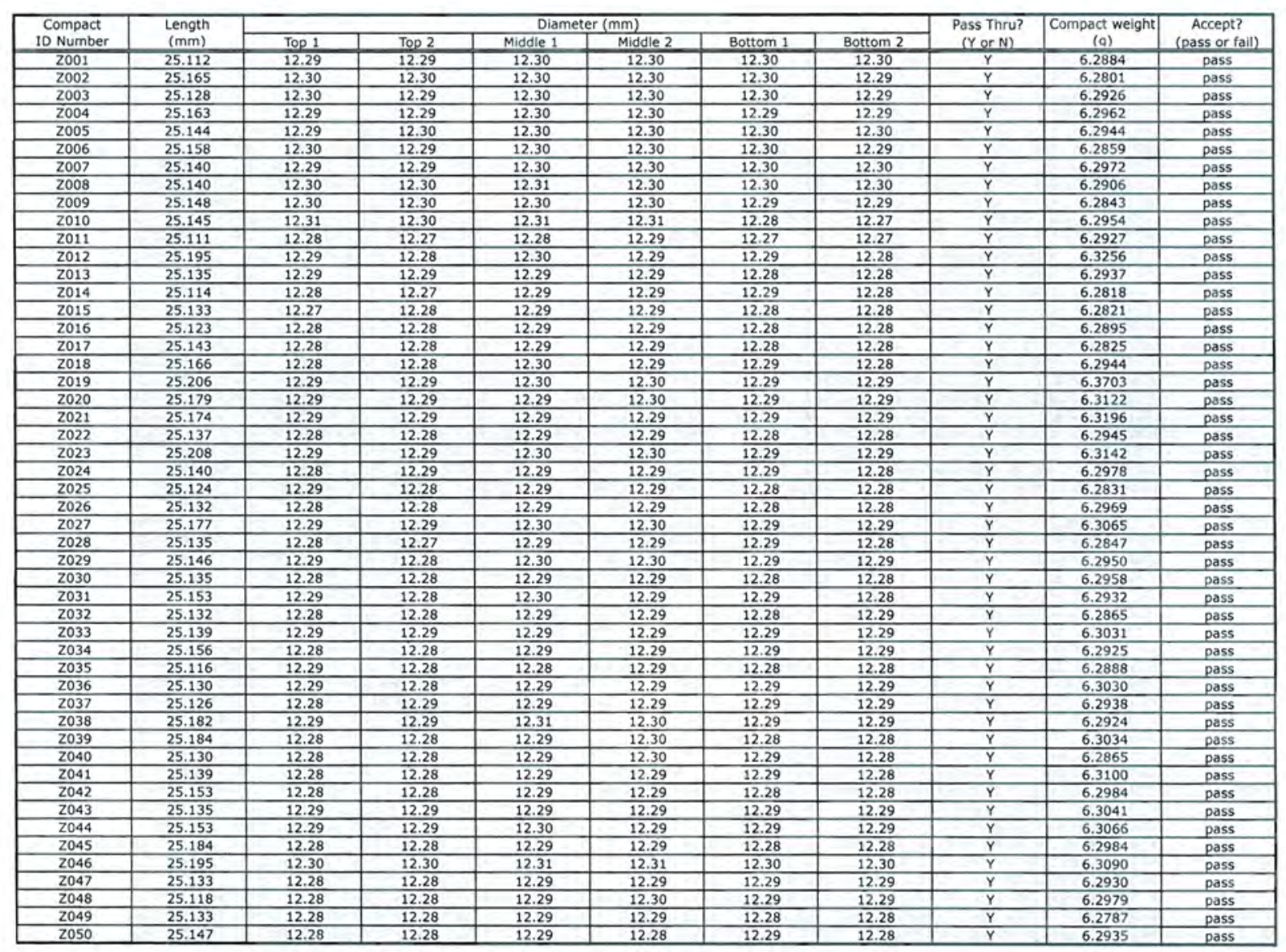

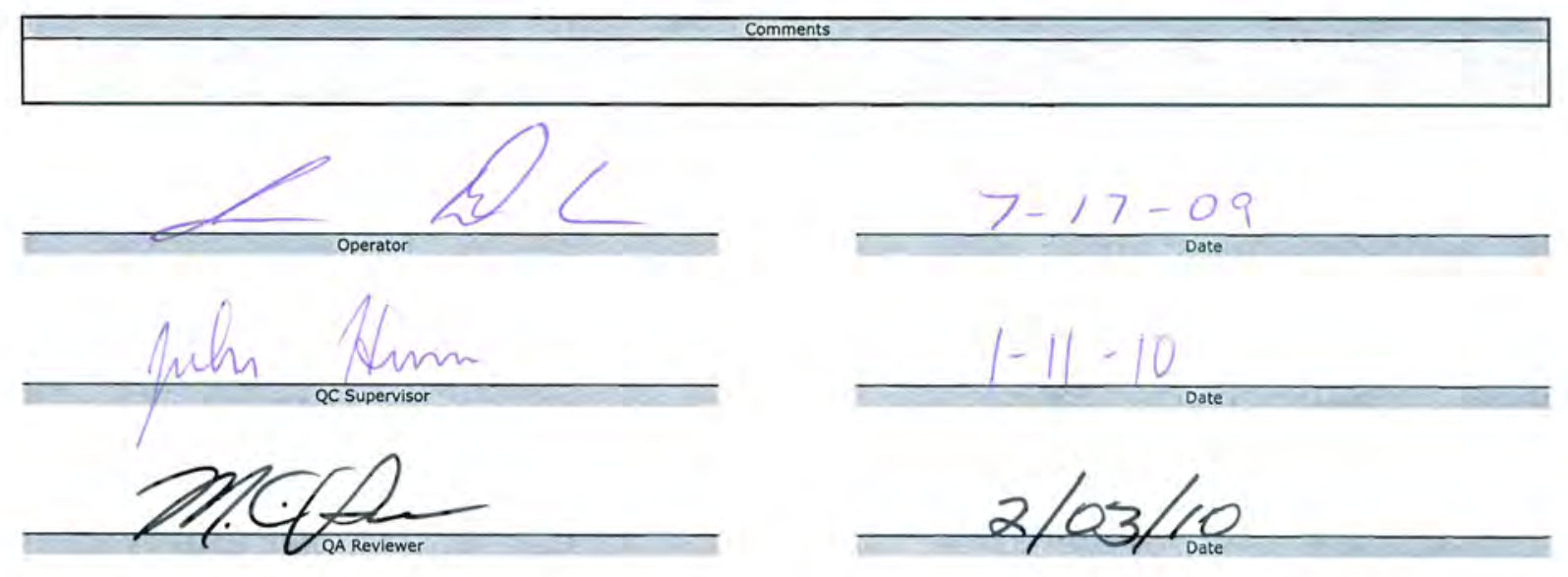

35 
ORNL/TM-2010/296

Data Report Form DRF-24A: Compact Diameter and Length

Procedure: AGR-CHAR-DAM-24 Rev. $6 \mathrm{a}$

Operator: Hunn/Barker/Dunbar Compact lot ID: $L$ LEU 09-OP2

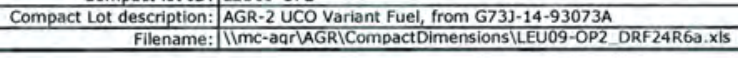

Vertical height gauge calibration due date: $3 / 6 / 10$

Pass-thru block calibration due date: $1 / 17 / 11$

Digital caliper calibration due date: $7 / 7 / 10$

Analytical balance calibration due date: $2 / 12 / 10 \& 10 / 29 / 09$

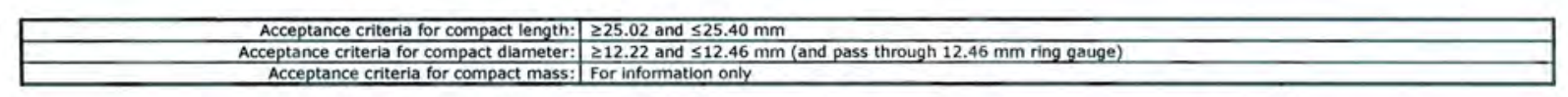

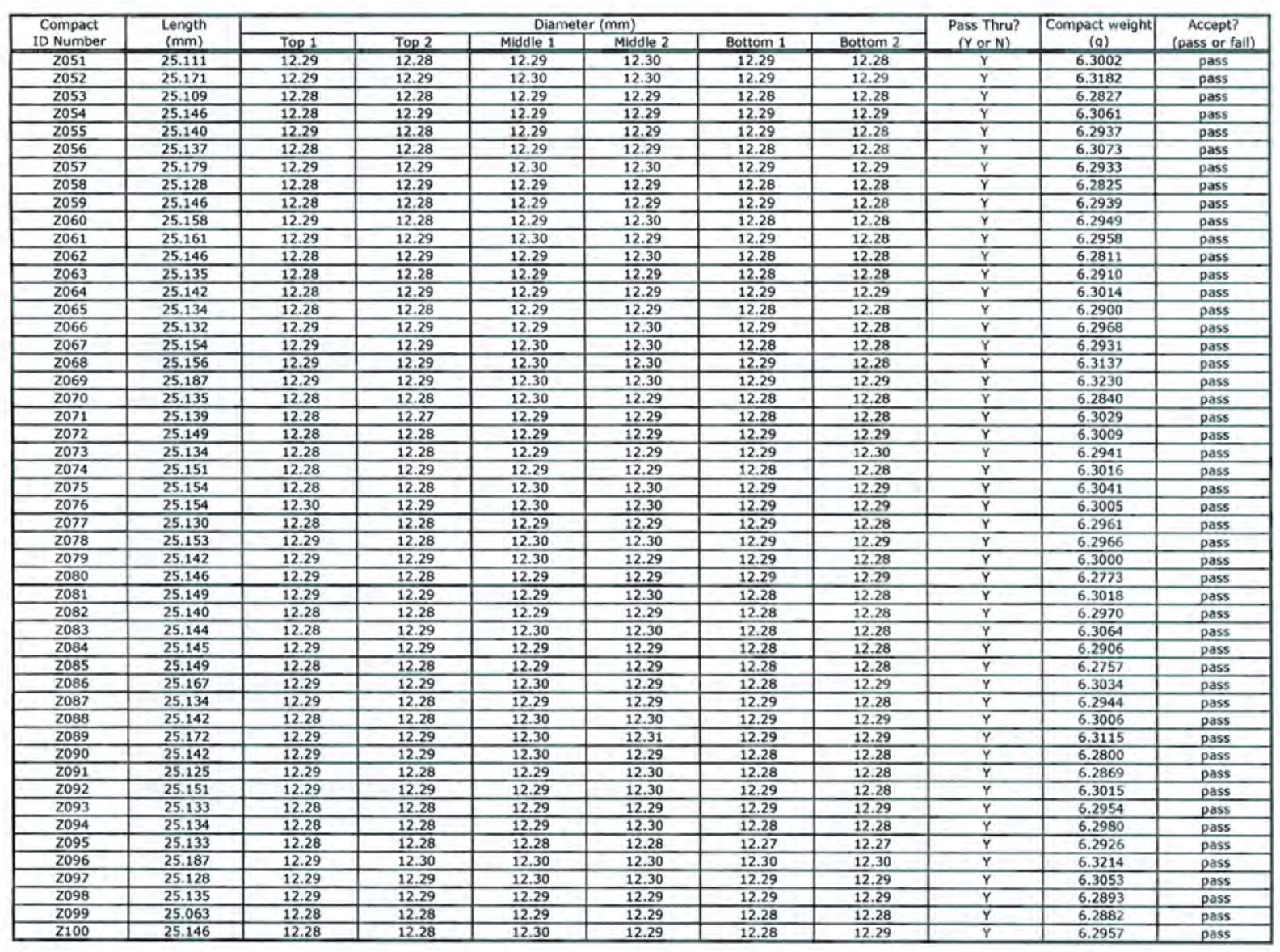
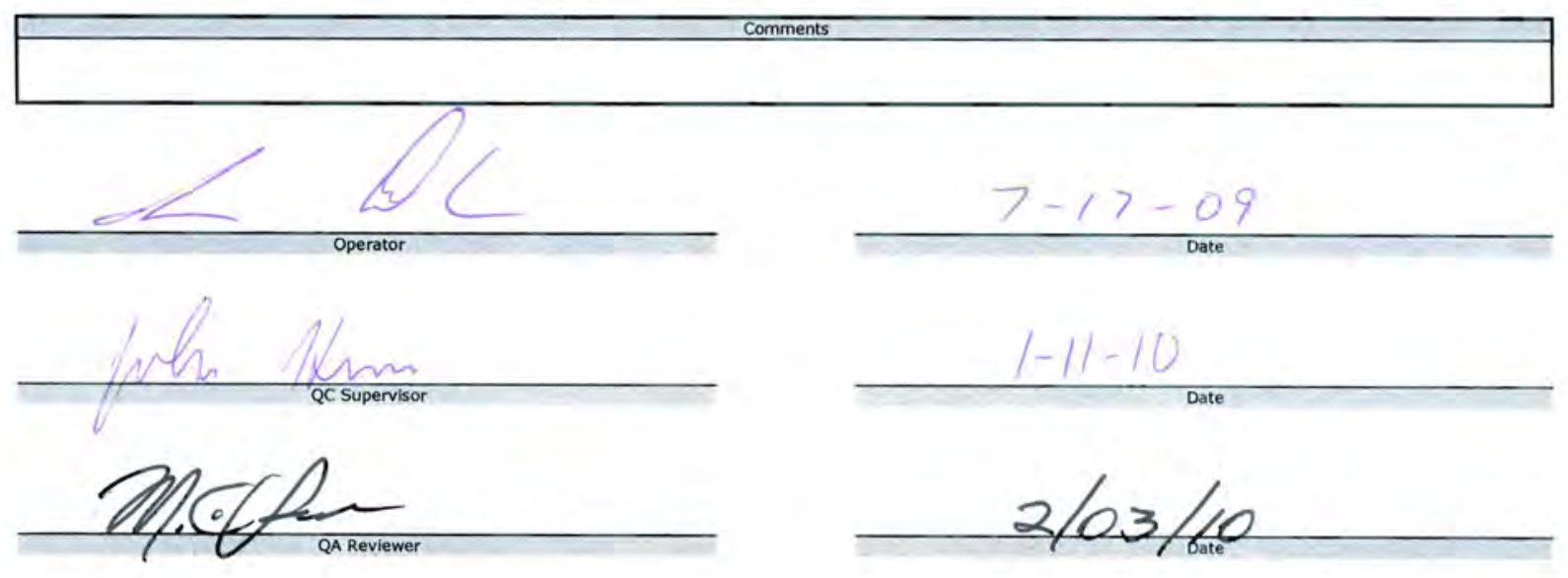

36 


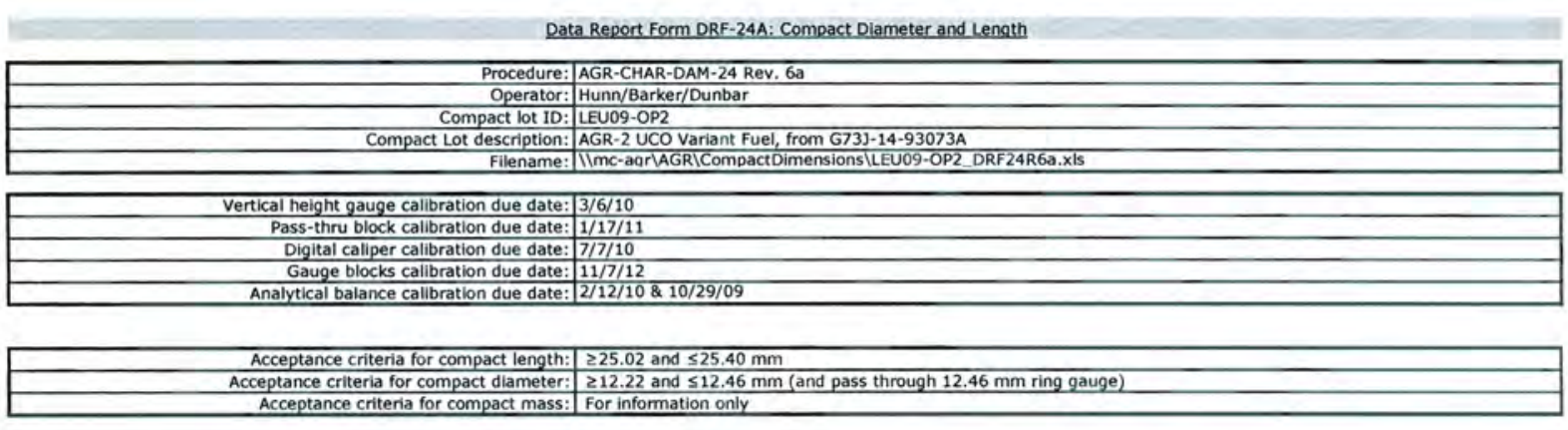

\begin{tabular}{|c|c|c|c|c|c|c|c|c|c|c|}
\hline Compact & Length & \multicolumn{6}{|c|}{ Diameter $(\mathrm{mm})$} & Pass Thru? & Compact weight & Accept? \\
\hline ID Number & $(\mathrm{mm})$ & Top 1 & Top 2 & Middle 1 & Middle 2 & Bottom 1 & Bottom 2 & $(Y \circ$ or $N)$ & (a) & (pass or fail) \\
\hline 2101 & 25.129 & 12.29 & 12.29 & 12.30 & 12.30 & 12.29 & 12.28 & $\frac{Y}{Y}$ & $\frac{6.3040}{6.2944}$ & pass \\
\hline$\frac{\mathrm{z} 102}{\mathrm{z} 103}$ & $\frac{25.125}{25.149}$ & $\frac{12.28}{12.28}$ & $\frac{12.28}{12.29}$ & $\frac{12.30}{12.28}$ & $\frac{12.30}{12.28}$ & $\frac{12.29}{12.28}$ & $\frac{12.28}{12.28}$ & $\frac{y}{y}$ & $\frac{6.2944}{6.859}$ & $\begin{array}{l}\text { pass } \\
\text { pass }\end{array}$ \\
\hline Z104 & 25.153 & 12.28 & 12.28 & 12.29 & 12.29 & 12.28 & 12.28 & $\bar{Y}$ & $\frac{0.2059}{6.3137}$ & pass \\
\hline $\mathrm{Z105}$ & 25.115 & 12.29 & 12.29 & 12.30 & 12.30 & 12.28 & 12.28 & $Y$ & 6.2989 & pass \\
\hline $\mathrm{Z} 106$ & 25.120 & 12.28 & 12.28 & 12.29 & 12.29 & 12.28 & 12.28 & Y & 6.2897 & pass \\
\hline $\mathrm{Z} 107$ & 25.151 & 12.29 & 12.29 & 12.29 & 12.29 & 12.29 & 12.28 & r & 6.2884 & pass \\
\hline Z108 & 25.024 & 12.28 & 12.27 & 12.28 & 12.29 & 12.27 & 12.28 & $\gamma$ & 6.2840 & pass \\
\hline 2109 & 25.152 & 12.29 & 12.29 & 12.29 & 12.29 & 12.28 & 12.28 & r & 6.2941 & pass \\
\hline $\mathrm{Z} 110$ & 25.151 & 12.28 & 12.28 & 12.29 & 12.29 & 12.28 & 12.28 & $y$ & 6.2837 & pass \\
\hline $\mathrm{Z} 111$ & 25.152 & 12.29 & 12.28 & 12.29 & 12.29 & 12.28 & 12.28 & y & 6.2904 & pass \\
\hline 2112 & 25.134 & 12.29 & 12.29 & 12.29 & 12.29 & 12.28 & 12.28 & $\begin{aligned} r & \end{aligned}$ & 6.2922 & pass \\
\hline 2113 & 25.114 & 12.28 & 12.28 & 12.29 & 12.29 & 12.28 & 12.29 & Y & 6.2861 & pass \\
\hline $\mathrm{Z} 114$ & 25.130 & 12.28 & 12.28 & 12.29 & 12.29 & 12.29 & 12.29 & $\begin{array}{r}y \\
\end{array}$ & 6.2934 & pass \\
\hline 2115 & 25.132 & 12.28 & 12.28 & 12.29 & 12.29 & 12.28 & 12.28 & $\begin{aligned} y & \end{aligned}$ & 6.2832 & pass \\
\hline 2116 & 25.142 & 12.29 & 12.28 & 12.29 & 12.29 & 12.28 & 12.29 & $\begin{array}{r}Y \\
Y\end{array}$ & 6.2870 & pass \\
\hline Z117 & 25.148 & 12.29 & 12.29 & 12.29 & 12.29 & 12.28 & 12.29 & Y & 6.3025 & pass \\
\hline 2118 & 25.151 & 12.28 & 12.28 & 12.29 & 12.30 & 12.28 & 12.28 & $r$ & 6.2928 & pass \\
\hline 2119 & 25.158 & 12.28 & 12.28 & 12.30 & 12.30 & 12.29 & 12.29 & $\begin{array}{r}Y \\
\end{array}$ & 6.2985 & pass \\
\hline 2120 & 25.120 & 12.29 & 12.29 & 12.30 & 12.30 & 12.28 & 12.29 & Y & 6.3053 & pass \\
\hline 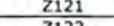 & 25.135 & 12.29 & $\frac{12.28}{128}$ & 12.29 & 12.29 & 12.29 & 12.28 & $\begin{array}{r}y \\
\end{array}$ & 6.2959 & pass \\
\hline$\frac{2122}{1723}$ & 25.130 & 12.28 & 12.28 & 12.30 & 12.29 & 12.28 & 12.28 & $Y$ & 6.2843 & pass \\
\hline$\frac{2123}{7124}$ & 25.151 & 12.28 & 12.28 & 12.29 & 12.29 & 12.29 & 12.29 & $Y$ & 6.2986 & pass \\
\hline$\frac{124}{8725}$ & $\frac{23.130}{25.130}$ & 12.29 & $\frac{12.29}{1428}$ & 12.29 & 12.29 & $\frac{12.28}{1428}$ & 12.28 & $\frac{r}{y}$ & 6.2830 & pass \\
\hline$\frac{1123}{7125}$ & $\frac{23.139}{251.34}$ & 12.49 & 12.28 & 12.29 & 12.30 & 12.28 & 12.29 & $\frac{r}{4}$ & 6.3026 & pass \\
\hline$\frac{1420}{7127}$ & $\frac{3.134}{25.12}$ & 12.28 & 12.280 & $\frac{12.30}{1.30}$ & 12.30 & $\frac{12.28}{1228}$ & 12.29 & $r$ & 6.2944 & pass \\
\hline 2128 & $\frac{35.142}{25.140}$ & $\frac{12.20}{1228}$ & $\frac{12.20}{1.28}$ & $\frac{12.29}{1620}$ & 12.30 & 12.20 & 12.200 & $r$ & 0.2080 & pass \\
\hline$\frac{1280}{7129}$ & $\frac{25.440}{25.130}$ & $\frac{12.20}{128}$ & $\frac{12.280}{1.28}$ & $\frac{12.25}{1.230}$ & 12.29 & 12.280 & 12.29 & $r$ & 0.2880 & pass \\
\hline$\frac{129}{7130}$ & $\frac{25.359}{25.132}$ & 12.20 & 12.68 & 12.30 & 12.300 & 12.29 & 12.29 & $\frac{y}{y}$ & 0.2387 & pass \\
\hline $\mathrm{Z131}$ & 25.142 & 1228 & 1228 & 1229 & 1279 & 1228 & 1228 & Y & $\frac{0.2535}{6204}$ & pass \\
\hline $\mathrm{Z132}$ & 25.217 & 12.28 & 12.28 & 12.30 & 12.29 & 12.27 & 12.28 & $\begin{array}{r}Y \\
Y\end{array}$ & 6.3422 & pass \\
\hline Z133 & 25.133 & 12.29 & 12.28 & 12.29 & 12.29 & 12.29 & 12.28 & $\bar{Y}$ & 6.2959 & pass \\
\hline Z134 & 25.147 & 12.29 & 12.29 & 12.30 & 12.30 & 12.29 & 12.28 & 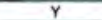 & 6.2983 & pass \\
\hline Z135 & 25.133 & 12.28 & 12.28 & 12.29 & 12.29 & 12.28 & 12.28 & $Y$ & 6.2972 & pass \\
\hline Z136 & 25,140 & 12.29 & 12.28 & 12.29 & 12.29 & 12.28 & 12.29 & Y & 6.2922 & pass \\
\hline Z137 & 25.144 & 12.29 & 12.28 & 12.29 & 12.30 & 12.28 & 12.28 & $\gamma$ & 6.2877 & pass \\
\hline Z138 & 25.177 & 12.29 & 12.29 & 12.30 & 12.30 & 12.29 & 12.28 & Y & 6.3043 & pass \\
\hline Z139 & 25.112 & 12.28 & 12.27 & 12.29 & 12.29 & 12.28 & 12.28 & $\gamma$ & 6.2862 & pass \\
\hline $\mathrm{Z140}$ & 25.161 & 12.28 & 12.28 & 12.29 & 12.29 & 12.29 & 12.29 & Y & 6.2964 & pass \\
\hline $\mathrm{Z} 141$ & 25.132 & 12.28 & 12.28 & 12.29 & 12.29 & 12.28 & 12.28 & Y & 6.2819 & pass \\
\hline 2142 & 25.114 & 12.28 & 12.28 & 12.29 & 12.30 & 12.28 & 12.28 & $\begin{array}{r}Y \\
\end{array}$ & 6.2926 & pass \\
\hline 2143 & 25.148 & 12.29 & 12.29 & 12.29 & 12.28 & 12.28 & 12.28 & Y & 6.2894 & pass \\
\hline 2144 & 25.156 & 12.28 & 12.28 & 12.29 & 12.29 & 12.28 & 12.27 & Y & 6.2958 & pass \\
\hline $\mathrm{Z} 145$ & 25.134 & 12.28 & 12.29 & 12.29 & 12.29 & 12.28 & 12.28 & Y & 6.2905 & pass \\
\hline 2146 & 25.163 & 12.29 & 12.28 & 12.30 & 12.29 & 12.28 & 12.29 & y & 6.3008 & pass \\
\hline 2147 & 25.158 & 12.29 & 12.29 & 12.30 & 12.30 & 12.28 & 12.28 & y & 6.3024 & pass \\
\hline 2148 & 23.142 & 12.28 & 12.28 & 12.29 & 12.30 & 12.28 & 12.29 & $\begin{array}{r}Y \\
\end{array}$ & 6.2992 & pass \\
\hline$\frac{2149}{7150}$ & 25.115 & $\frac{12.28}{1.20}$ & 12.28 & $\frac{12.29}{1.20}$ & $\frac{12.30}{1.320}$ & 12.28 & 12.28 & $\begin{array}{r}y \\
\end{array}$ & 6.2842 & pass \\
\hline Z150 & 25.148 & 12.29 & 12.28 & 12.30 & 12.30 & 12.29 & 12.28 & y & 6.2950 & pass \\
\hline
\end{tabular}
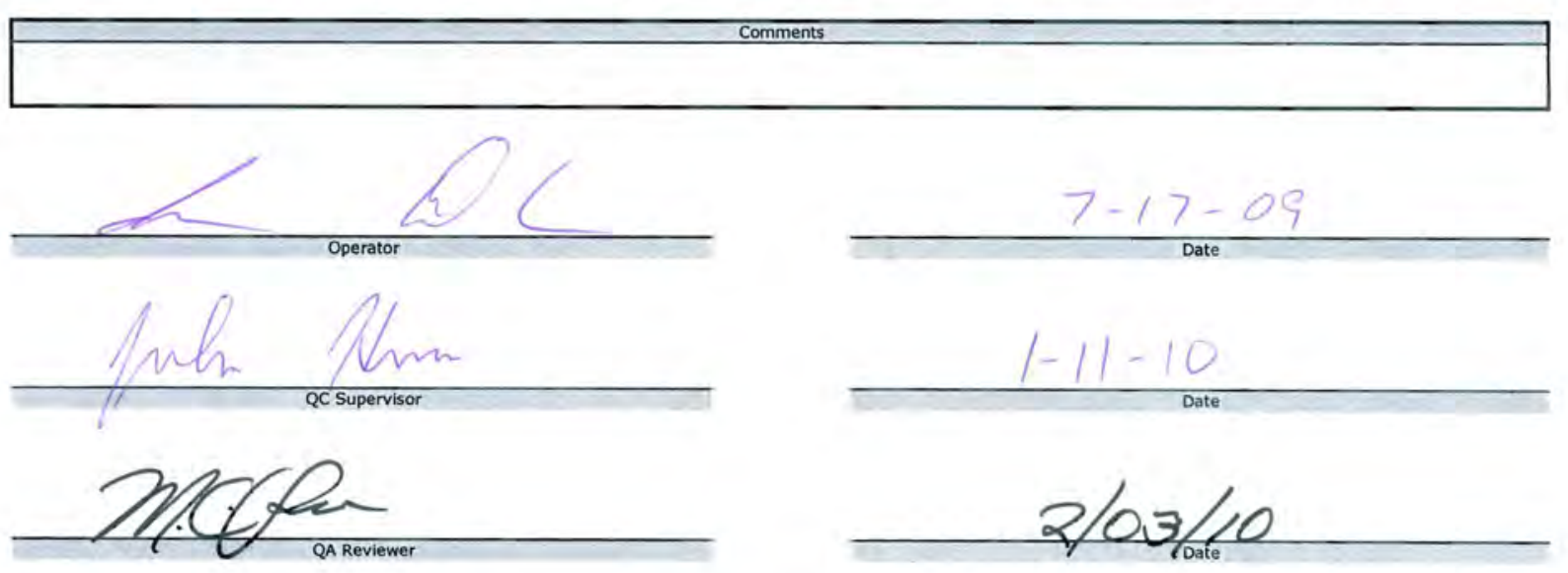
ORNL/TM-2010/296
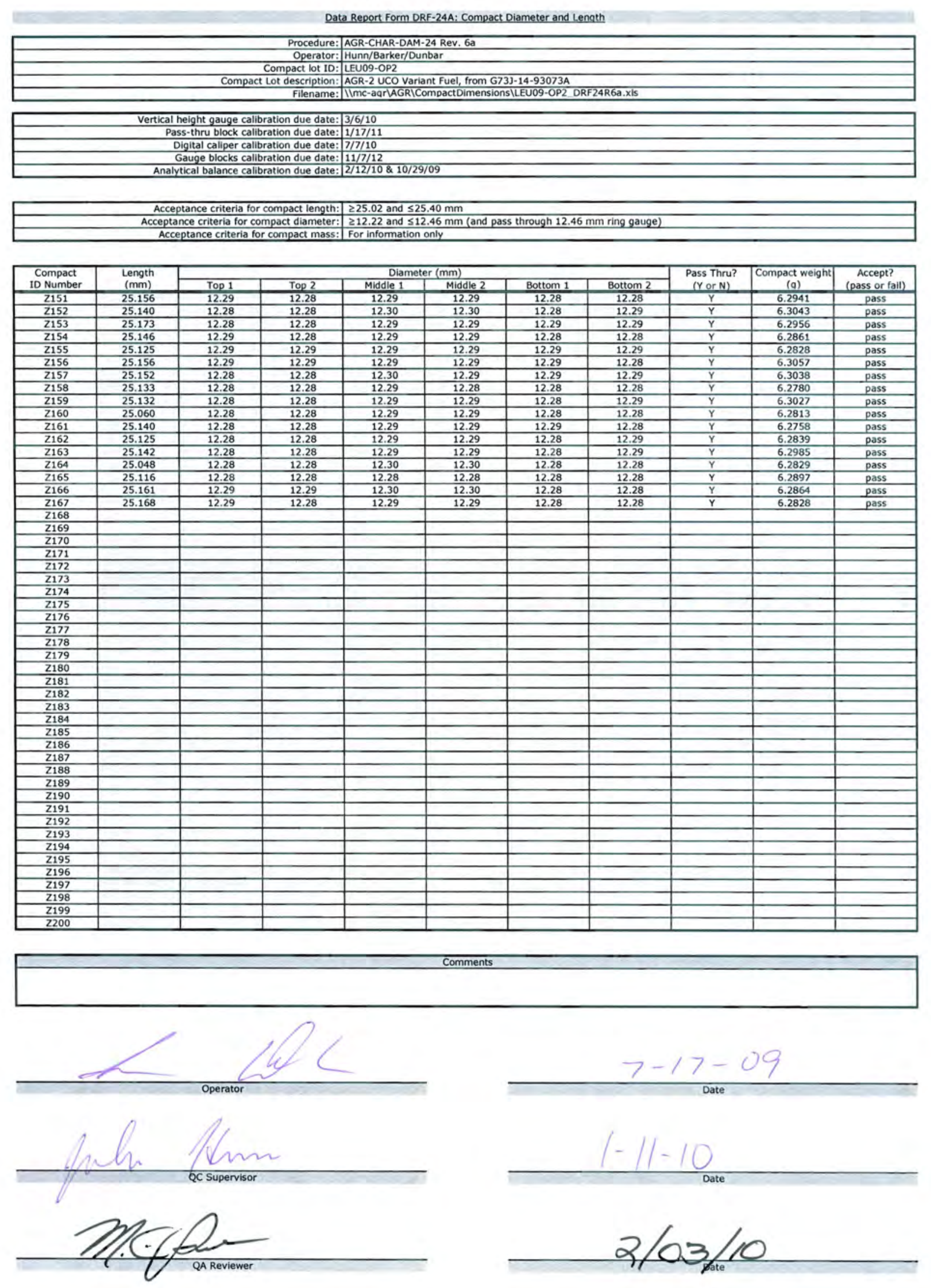

38 
Data Report Form DRF-24B: Compact Matrix Density

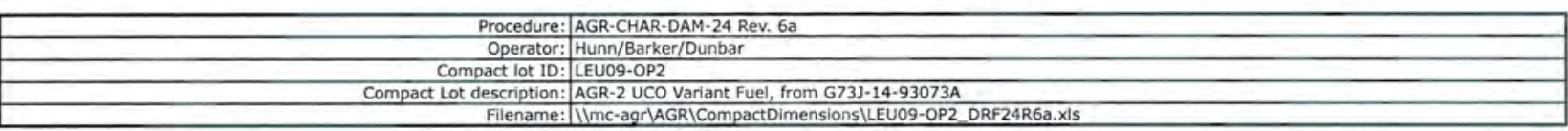

\begin{tabular}{|c|c|}
\hline Average weight per TRISO particle $(g):$ & $1.032 \mathrm{E}-03$ \\
\hline Average weight per overcoated particle $(\mathrm{g}):$ & $2.010 \mathrm{E}-03$ \\
\hline Average TRISO particle volume $(\mathrm{cm} 3): 3.430 \mathrm{E}-04$ \\
\hline
\end{tabular}

\section{Acceptance criteria for matrix density: $: \geq 1.45$}

\begin{tabular}{|c|c|c|c|c|c|c|c|c|c|c|}
\hline $\begin{array}{l}\text { Compact } \\
\text { ID Number }\end{array}$ & $\begin{array}{l}\text { Compact Weight } \\
\text { (a) }\end{array}$ & $\begin{array}{l}\text { Length } \\
\text { (mm) }\end{array}$ & $\begin{array}{c}\text { Av. Diameter } \\
(\mathrm{mm})\end{array}$ & \begin{tabular}{|c|} 
Compact Volume \\
$(\mathrm{cm} 3)$
\end{tabular} & $\begin{array}{l}\text { Charge Weight } \\
\text { (a) }\end{array}$ & $\begin{array}{l}\text { Particle Weight } \\
\text { (a) }\end{array}$ & $\begin{array}{c}\text { Particle Volume } \\
(\mathrm{cm} 3)\end{array}$ & Packing Fraction & $\begin{array}{l}\text { Matrix Density } \\
(\mathrm{a} / \mathrm{cm} 3)\end{array}$ & $\begin{array}{c}\text { Accept? } \\
\text { (pass or fail) }\end{array}$ \\
\hline 2001 & \begin{tabular}{|l|l|}
6.2884 \\
\end{tabular} & 25.112 & 12.30 & 2.98 & 6.4327 & 3.3024 & 1.10 & $37 \%$ & 1.58 & pass \\
\hline 2002 & 6.2801 & 25.165 & 12.30 & 2.99 & 6.4306 & 3.3014 & 1.10 & $37 \%$ & 1.57 & pass \\
\hline 2003 & 6.2926 & 25.128 & 12.30 & 2.98 & 6.4303 & 3.3014 & 1.10 & $37 \%$ & 1.59 & pass \\
\hline 2004 & 6.2962 & 25.163 & 12.29 & 2.99 & 6.4318 & 3.3024 & 1.10 & $37 \%$ & 1.58 & pass \\
\hline 2005 & 6.2944 & 25.144 & 12.30 & 2.99 & 6.4309 & 3.3014 & 1.10 & $37 \%$ & 1.58 & pass \\
\hline 2006 & 6.2859 & 25.158 & 12.30 & 2.99 & 6.4313 & 3.3024 & 1.10 & $37 \%$ & 1.58 & pass \\
\hline 2007 & 6.2972 & 25.140 & 12.30 & 2.99 & 6.4311 & 3.3024 & 1.10 & $37 \%$ & 1.59 & pass \\
\hline 2008 & 6.2906 & 25.140 & 12.30 & 2.99 & 6.4318 & 3.3024 & 1.10 & $37 \%$ & 1.58 & pass \\
\hline 2009 & 6.2843 & 25.148 & 12.30 & 2.99 & 6.4326 & 3.3024 & 1.10 & $37 \%$ & 1.58 & pass \\
\hline 2010 & 6.2954 & 25.145 & 12.30 & 2.99 & 6.4322 & 3.3024 & 1.10 & $37 \%$ & 1.58 & pass \\
\hline Z011 & 6.2927 & 25.111 & 12.28 & 2.97 & 6.4307 & 3.3014 & 1.10 & $37 \%$ & 1.60 & pass \\
\hline 2012 & 6.3256 & 25.195 & 12.29 & 2.99 & 6.4329 & 3.3024 & 1.10 & $37 \%$ & 1.60 & pass \\
\hline 2013 & 6.2937 & 25.135 & 12.29 & 2.98 & 6.4313 & 3.3024 & 1.10 & $37 \%$ & 1.59 & pass \\
\hline 2014 & 6.2818 & 25.114 & 12.28 & 2.98 & 6.4311 & 3.3024 & 1.10 & $37 \%$ & 1.59 & pass \\
\hline 2015 & 6.2821 & 25.133 & 12.28 & 2.98 & 6.4325 & 3.3024 & 1.10 & $37 \%$ & 1.59 & pass \\
\hline 2016 & 6.2895 & 25.123 & 12.28 & 2.98 & 6.4315 & 3.3024 & 1.10 & $37 \%$ & 1.59 & pass \\
\hline 2017 & 6.2825 & 25.143 & 12.28 & 2.98 & 6.4322 & 3.3024 & 1.10 & $37 \%$ & 1.58 & pass \\
\hline 2018 & 6.2944 & 25.166 & 12.29 & 2.98 & 6.4329 & 3.3024 & 1.10 & $37 \%$ & 1.59 & pass \\
\hline 2019 & 6.3703 & 25.206 & 12.29 & 2.99 & 6.4325 & 3.3024 & 1.10 & $37 \%$ & 1.62 & pass \\
\hline 2020 & 6.3122 & 25.179 & 12.29 & 2.99 & 6.4312 & 3.3024 & 1.10 & $37 \%$ & 1.59 & pass \\
\hline 2021 & 6.3196 & 25.174 & 12.29 & 2.99 & 6.4323 & 3.3024 & 1.10 & $37 \%$ & 1.60 & pass \\
\hline 2022 & 6.2945 & 25.137 & 12.28 & 2.98 & 6.4311 & 3.3024 & 1,10 & $37 \%$ & 1.59 & pass \\
\hline 2023 & 6.3142 & 25.208 & 12.29 & 2.99 & 6.4314 & 3.3024 & 1.10 & $37 \%$ & 1.59 & $\frac{p \text { pass }}{\text { pass }}$ \\
\hline 2024 & 6.2978 & 25.140 & 12.29 & 2.98 & 6.4302 & 3.3014 & 1.10 & $37 \%$ & 1.59 & pass \\
\hline 2025 & 6.2831 & 25.124 & 12.29 & 2.98 & 6.4302 & 3.3014 & 1.10 & $37 \%$ & 1.59 & pass \\
\hline 2026 & 6.2969 & 25.132 & 12.28 & 2.98 & 6.4314 & 3.3024 & 1.10 & $37 \%$ & 1.59 & pass \\
\hline 2027 & 6.3065 & 25.177 & 12.29 & 2.99 & 6.4320 & 3.3024 & 1.10 & $37 \%$ & 1.59 & pass \\
\hline 2028 & 6.2847 & 25.135 & 12.28 & 2.98 & 6.4320 & 3.3024 & 1.10 & $37 \%$ & 1.59 & pass \\
\hline 2029 & 6.2950 & 25.146 & 12.29 & 2.98 & 6.4328 & 3.3024 & 1.10 & $37 \%$ & 1.59 & pass \\
\hline 2030 & 6.2958 & 25.135 & 12.28 & 2.98 & 6.4304 & 3.3014 & 1.10 & $37 \%$ & 1.59 & pass \\
\hline 2031 & 6.2932 & 25.153 & 12.29 & 2.98 & 6.4321 & 3.3024 & 1.10 & $37 \%$ & 1.59 & pass \\
\hline 2032 & 6.2865 & 25.132 & 12.29 & 2.98 & 6.4308 & 3.3014 & 1.10 & $37 \%$ & 1.59 & pass \\
\hline 2033 & 6.3031 & 25.139 & 12.29 & 2.98 & 6.4326 & 3.3024 & 1.10 & $37 \%$ & 1.59 & pass \\
\hline 2034 & 6.2925 & 25.156 & 12.29 & 2.98 & 6.4325 & 3.3024 & 1.10 & $37 \%$ & 1.59 & pass \\
\hline 2035 & 6.2888 & 25.1116 & 12.28 & 2.98 & 6.4310 & 3.3024 & 1.10 & $37 \%$ & 1.59 & pass \\
\hline 2036 & 6.3030 & 25.130 & 12.29 & 2.98 & 6.4326 & 3.3024 & 1.10 & $37 \%$ & 1.59 & pass \\
\hline 2037 & 6.2938 & 25.126 & 12.29 & 2.98 & 6.4309 & 3.3014 & 1.10 & $37 \%$ & 1.59 & pass \\
\hline 2038 & 6.2924 & 25.182 & 12,30 & 2.99 & 6.4326 & 3.3024 & 1.10 & $37 \%$ & 1.58 & $\begin{array}{l}\text { pass } \\
\text { pass }\end{array}$ \\
\hline 2039 & 6.3034 & 25.184 & 12.29 & 2.99 & 6.4310 & 3.3024 & 1.10 & $37 \%$ & 1.59 & pass \\
\hline 2040 & 6.2865 & 25.130 & 12.29 & 2.98 & 6.4327 & 3.3024 & 1.10 & $37 \%$ & 1.59 & pass \\
\hline 2041 & $\frac{6.2605}{6.3100}$ & 25.139 & $\frac{12.29}{12.29}$ & $\frac{2.90}{2.98}$ & 6.4304 & $\begin{array}{l}3.3524 \\
3.3014\end{array}$ & 1.10 & $37 \%$ & 1.60 & $\frac{\text { pass }}{\text { pass }}$ \\
\hline$\frac{041}{\mathrm{Z} 042}$ & $\frac{6.3100}{6.2984}$ & $\frac{25.159}{25.153}$ & $\frac{12.29}{12.28}$ & $\frac{2.98}{2.98}$ & $\begin{array}{l}6.4040 \\
6.4329\end{array}$ & $\frac{3.3014}{3.3024}$ & $\frac{1.10}{1.10}$ & $37 \%$ & $\begin{array}{l}1.60 \\
1.59\end{array}$ & $\begin{array}{l}\text { pass } \\
\text { pass }\end{array}$ \\
\hline 2043 & 6.3041 & 25.135 & 12.29 & 2.98 & 6.4320 & 3.3024 & 1.10 & $37 \%$ & 1.59 & pass \\
\hline 2044 & 6.3066 & 25.153 & 12.29 & 2.98 & 6.4309 & 3.3014 & 1.10 & $37 \%$ & 1.59 & pass \\
\hline Z045 & 6.2984 & 25.184 & 12.28 & 2.98 & 6.4311 & 3.3024 & 1.10 & $37 \%$ & 1.59 & pass \\
\hline 2046 & 6.3090 & 25.195 & 12,30 & 3.00 & 6.4311 & 3.3024 & 1.10 & $37 \%$ & 1.58 & pass \\
\hline 2047 & 6.2930 & 25.133 & 12.29 & 2.98 & 6.4316 & 3.3024 & 1.10 & $37 \%$ & 1.59 & pass \\
\hline 2048 & 6.2979 & 25.118 & 12.29 & 2.98 & 6.4306 & 3.3014 & 1.10 & & & pass \\
\hline 2049 & 6.2787 & 25.133 & 12.28 & $\frac{2.30}{2.98}$ & 6.4333 & 3.3034 & 1.10 & $37 \%$ & 1.58 & pass \\
\hline 2050 & 6.2935 & 25.147 & 12.28 & 2.98 & 6.4318 & 3.3024 & 1.10 & $37 \%$ & 1.59 & pass \\
\hline
\end{tabular}
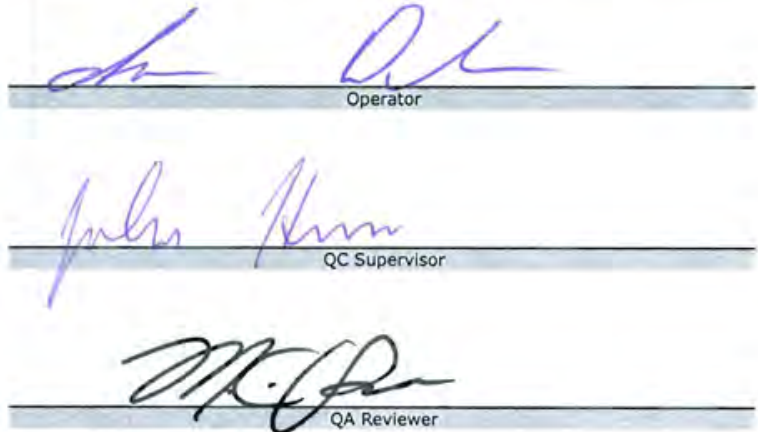

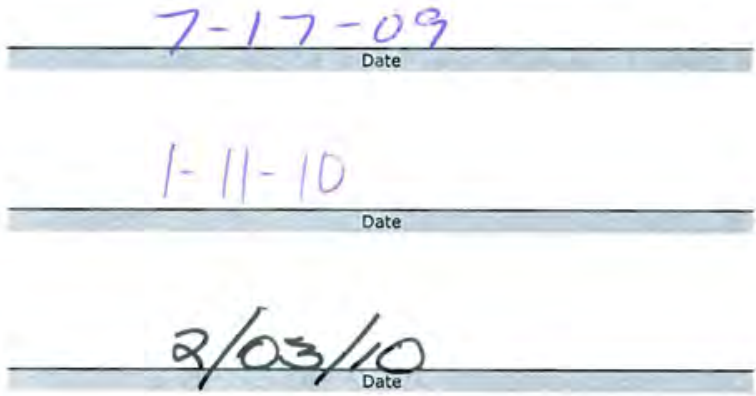


Data Report Form DRF-248: Compact Matrix Density

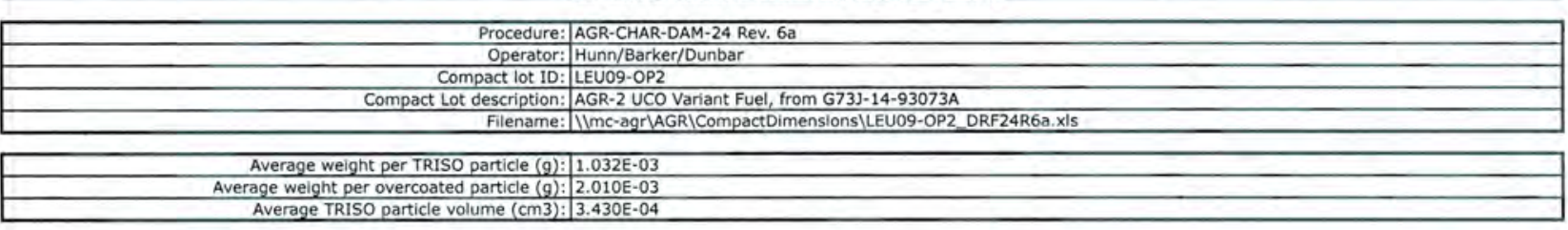
Acceptance criteria for matrix density: $\mid \geq 1.45$

\begin{tabular}{|c|c|c|c|c|c|c|c|c|c|c|}
\hline $\begin{array}{c}\text { Compact } \\
\text { ID Number }\end{array}$ & \begin{tabular}{|c|} 
Compact Weight \\
(a)
\end{tabular} & $\begin{array}{l}\text { Length } \\
\text { (mm) }\end{array}$ & $\begin{array}{l}\text { Av. Diameter } \\
\text { (mm) }\end{array}$ & \begin{tabular}{|c|} 
Compact Volume \\
$(\mathrm{cm} 3)$
\end{tabular} & $\begin{array}{l}\text { Charge Weight } \\
\text { (a) }\end{array}$ & $\begin{array}{c}\text { Particle Weight } \\
\text { (a) }\end{array}$ & $\begin{array}{l}\text { Particle Volume } \\
(\mathrm{cm} 3)\end{array}$ & Packing Fraction & $\begin{array}{c}\text { Matrix Density } \\
(\mathrm{a} / \mathrm{cm} 3)\end{array}$ & $\begin{array}{c}\text { Accept? } \\
\text { (pass or fail) }\end{array}$ \\
\hline 2051 & 6.3002 & 25.111 & 12.29 & 2.98 & 6.4303 & 3.3014 & 1.10 & $37 \%$ & 1.59 & pass \\
\hline 2052 & 6.3182 & 25.171 & 12.29 & 2.99 & 6.4315 & 3.3024 & 1.10 & $37 \%$ & 1.60 & pass \\
\hline 2053 & 6.2827 & 25.109 & 12.28 & 2.98 & 6.4321 & 3.3024 & 1.10 & $37 \%$ & 1.59 & pass \\
\hline 2054 & 6.3061 & 25.146 & 12.29 & 2.98 & 6.4316 & 3.3024 & 1.10 & $37 \%$ & 1.59 & pass \\
\hline 2055 & 6.2937 & 25.140 & 12.29 & 2.98 & 6.4321 & 3.3024 & 1.10 & $37 \%$ & 1.59 & pass \\
\hline 2056 & 6.3073 & 25.137 & 12.28 & 2.98 & 6.4316 & 3.3024 & 1.10 & $37 \%$ & 1.60 & pass \\
\hline 2057 & 6.2933 & 25.179 & 12.29 & 2.99 & 6.4309 & 3.3014 & 1.10 & $37 \%$ & 1.58 & pass \\
\hline 2058 & 6.2825 & 25.128 & 12.29 & 2.98 & 6.4295 & 3.3014 & 1.10 & $37 \%$ & 1.58 & pass \\
\hline 2059 & 6.2939 & 25.146 & 12.29 & 2.98 & 6.4307 & 3.3014 & 1.10 & $37 \%$ & 1.59 & pass \\
\hline 2060 & 6.2949 & 25.158 & 12.29 & 2.98 & 6.4308 & 3.3014 & 1.10 & $37 \%$ & 1.59 & pass \\
\hline 2061 & 6.2958 & 25.161 & 12.29 & 2.98 & 6.4323 & 3.3024 & 1.10 & $37 \%$ & 1.59 & pass \\
\hline 2062 & 6.2811 & 25.146 & 12.29 & 2.98 & 6.4306 & 3.3014 & 1.10 & $37 \%$ & 1.58 & pass \\
\hline 2063 & 6.2910 & 25.135 & 12.28 & 2.98 & 6.4314 & 3.3024 & 1.10 & $37 \%$ & 1.59 & pass \\
\hline 2064 & 6.3014 & 25.142 & 12.29 & 2.98 & 6.4314 & 3.3024 & 1.10 & $37 \%$ & 1.59 & pass \\
\hline 2065 & 6.2900 & 25.134 & 12.28 & 2.98 & 6.4321 & 3.3024 & 1.10 & $37 \%$ & 1.59 & pass \\
\hline 2066 & 6.2968 & 25.132 & 12.29 & 2.98 & 6.4300 & 3.3014 & 1.10 & $37 \%$ & 1.59 & pass \\
\hline 2067 & 6.2931 & 25.154 & 12.29 & 2.98 & 6.4311 & 3.3024 & 1.10 & $37 \%$ & 1.59 & pass \\
\hline 2068 & 6.3137 & 25.156 & 12.29 & 2.99 & 6.4325 & 3.3024 & 1.10 & $37 \%$ & 1.60 & pass \\
\hline 2069 & 6.3230 & 25.187 & 12.29 & 2.99 & 6.4325 & 3.3024 & 1.10 & $37 \%$ & 1.60 & pass \\
\hline 2070 & 6.2840 & 25.135 & 12.29 & 2.98 & 6.4324 & 3.3024 & 1.10 & $37 \%$ & 1.58 & pass \\
\hline 2071 & 6.3029 & 25.139 & 12.28 & 2.98 & 6.4326 & 3.3024 & 1.10 & $37 \%$ & 1.60 & pass \\
\hline 2072 & 6.3009 & 25.149 & 12.29 & 2.98 & 6.4323 & 3.3024 & 1.10 & $37 \%$ & 1.59 & pass \\
\hline 2073 & 6.2941 & 25.134 & 12.29 & 2.98 & 6.4311 & 3.3024 & 1.10 & $37 \%$ & 1.59 & pass \\
\hline 2074 & 6.3016 & 25.151 & 12.29 & 2.98 & 6.4308 & 3.3014 & 1.10 & $37 \%$ & 1.59 & pass \\
\hline 2075 & 6.3041 & 25.154 & 12.29 & 2.98 & 6.4309 & 3.3014 & 1.10 & $37 \%$ & 1.59 & pass \\
\hline 2076 & 6.3005 & 25.154 & 12.30 & 2.99 & 6.4331 & 3.3034 & 1.10 & $37 \%$ & 1.59 & pass \\
\hline 2077 & 6.2961 & 25.130 & 12.29 & 2.98 & 6.4319 & 3.3024 & 1.10 & $37 \%$ & 1.59 & pass \\
\hline 2078 & 6.2966 & 25.153 & 12.29 & 2.98 & 6.4323 & 3.3024 & 1.10 & $37 \%$ & 1.59 & pass \\
\hline 2079 & 6.3000 & 25.142 & 12.29 & 2.98 & 6.4323 & 3.3024 & 1.10 & $37 \%$ & 1.59 & pass \\
\hline 2080 & 6.2773 & 25.146 & 12.29 & 2.98 & 6.4322 & 3.3024 & 1.10 & $37 \%$ & 1.58 & pass \\
\hline 2081 & 6.3018 & 25.149 & 12.29 & 2.98 & 6.4314 & 3,3024 & 1.10 & $37 \%$ & 1.59 & pass \\
\hline 2082 & 6.2970 & 25.140 & 12.28 & 2.98 & 6.4313 & 3.3024 & 1.10 & $37 \%$ & 1.59 & pass \\
\hline 2083 & 6.3064 & 25.144 & 12.29 & 2.98 & 6.4302 & 3.3014 & 1.10 & $37 \%$ & 1.59 & pass \\
\hline 2084 & 6.2906 & 25.145 & 12.29 & 2.98 & 6.4325 & 3.3024 & 1.10 & $37 \%$ & 1.59 & pass \\
\hline 2085 & 6.2757 & 25.149 & 12.28 & 2.98 & 6.4303 & 3.3014 & 1.10 & $37 \%$ & 1.58 & pass \\
\hline 2086 & 6.3034 & 25.167 & 12.29 & 2.99 & 6.4322 & 3.3024 & 1.10 & $37 \%$ & 1.59 & pass \\
\hline 2087 & 6.2944 & 25.134 & 12.29 & 2.98 & 6.4328 & 3.3024 & 1.10 & $37 \%$ & 1.59 & pass \\
\hline 2088 & 6.3006 & 25.142 & 12.29 & 2.98 & 6.4320 & 3.3024 & 1.10 & $37 \%$ & 1.59 & pass \\
\hline 2089 & 6.3115 & 25.172 & 12.30 & 2.99 & 6.4304 & 3.3014 & 1.10 & $37 \%$ & 1.59 & pass \\
\hline 2090 & 6.2800 & 25.142 & 12.29 & 2.98 & 6.4321 & 3.3024 & 1.10 & $37 \%$ & 1.58 & pass \\
\hline 2091 & 6.2869 & 25.125 & 12.29 & 2.98 & 6.4302 & 3.3014 & 1.10 & $37 \%$ & 1.59 & pass \\
\hline 2092 & 6.3015 & 25.151 & 12.29 & 2.98 & 6.4317 & 3.3024 & 1.10 & $37 \%$ & 1.59 & pass \\
\hline 2093 & 6.2954 & 25.133 & 12.29 & 2.98 & 6.4310 & 3.3024 & 1.10 & $37 \%$ & 1.59 & pass \\
\hline 2094 & 6.2980 & 25.134 & 12.29 & 2.98 & 6.4314 & 3.3024 & 1.10 & $37 \%$ & 1.59 & pass \\
\hline 2095 & 6.2926 & 25.133 & 12.28 & 2.98 & 6.4306 & 3.3014 & 1.10 & $37 \%$ & 1.59 & pass \\
\hline 2096 & 6.3214 & 25.187 & 12.30 & 2.99 & 6.4322 & 3.3024 & 1.10 & $37 \%$ & 1.59 & pass \\
\hline 2097 & 6.3053 & 25.128 & 12.29 & 2.98 & 6.4321 & 3.3024 & 1.10 & $37 \%$ & 1.59 & pass \\
\hline 2098 & 6.2893 & 25.135 & 12.29 & 2.98 & 6.4316 & 3.3024 & 1.10 & $37 \%$ & 1.59 & pass \\
\hline 2099 & 6.2882 & 25.063 & 12.28 & 2.97 & 6.4321 & 3.3024 & 1.10 & $37 \%$ & 1.59 & pass \\
\hline 2100 & 6.2957 & 25.146 & 12.29 & 2.98 & 6.4321 & 3.3024 & 1.10 & $37 \%$ & 1.59 & pass \\
\hline
\end{tabular}
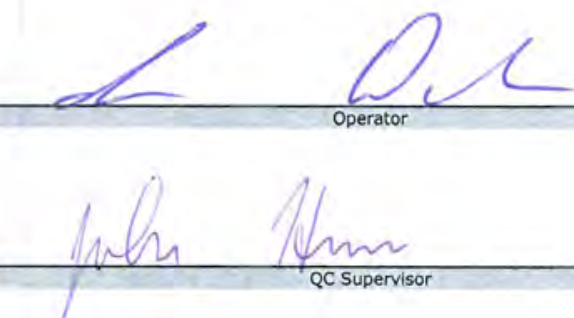

Mepar

$$
7-17-09
$$


Data Report Form DRF-24B: Compact Matrix Density

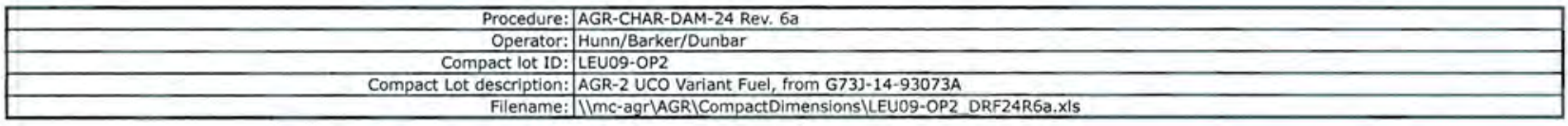

\begin{tabular}{|c|}
\hline Average weight per TRISO particle $(g): 1.032 \mathrm{E}-03$ \\
\hline Average weight per overcoated particle $(\mathrm{g}): 2.010 \mathrm{E}-03$ \\
\hline Average TRISO particle volume $(\mathrm{cm} 3): 13.430 \mathrm{E}-04$ \\
\hline
\end{tabular}

Acceptance criteria for matrix density: $\geq 1.45$

\begin{tabular}{|c|c|c|c|c|c|c|c|c|c|c|}
\hline $\begin{array}{l}\text { Compact } \\
\text { ID Number }\end{array}$ & $\begin{array}{l}\text { Compact Weight } \\
\text { (a) }\end{array}$ & $\begin{array}{l}\text { Length } \\
(\mathrm{mm})\end{array}$ & $\begin{array}{c}\text { Av. Diameter } \\
(\mathrm{mm})\end{array}$ & $\begin{array}{l}\text { Compact Volume } \\
(\mathrm{cm} 3)\end{array}$ & $\begin{array}{l}\text { Charge Weight } \\
\text { (a) }\end{array}$ & $\begin{array}{l}\text { Particle Weight } \\
\text { (a) }\end{array}$ & $\begin{array}{l}\text { Particle Volume } \\
(\mathrm{cm} 3)\end{array}$ & Packing Fraction & $\begin{array}{l}\text { Matrix Density } \\
(\mathrm{a} / \mathrm{cm} 3)\end{array}$ & $\begin{array}{c}\text { Accept? } \\
\text { (pass or fail) }\end{array}$ \\
\hline $\mathrm{Z} 101$ & 6.3040 & 25.129 & 12.29 & 2.98 & 6.4327 & 3.3024 & 1.10 & $37 \%$ & 1.59 & pass \\
\hline $\mathrm{Z102}$ & 6.2944 & 25.125 & 12.29 & 2.98 & 6.4319 & 3.3024 & 1.10 & $37 \%$ & 1.59 & pass \\
\hline $\mathrm{Z103}$ & 6.2859 & 25.149 & 12.28 & 2.98 & 6.4306 & 3.3014 & 1.10 & $37 \%$ & 1.59 & pass \\
\hline $\mathrm{Z} 104$ & 6.3137 & 25.153 & 12.28 & 2.98 & 6.4327 & 3.3024 & 1.10 & $37 \%$ & 1.60 & pass \\
\hline 2105 & 6.2989 & 25.115 & 12.29 & 2.98 & 6.4313 & 3.3024 & 1.10 & $37 \%$ & 1.59 & pass \\
\hline $\mathrm{Z106}$ & 6.2897 & 25.120 & 12.28 & 2.98 & 6.4314 & 3.3024 & 1.10 & $37 \%$ & 1.59 & pass \\
\hline $\mathrm{Z} 107$ & 6.2884 & 25.151 & 12.29 & 2.98 & 6.4299 & 3.3014 & 1.10 & $37 \%$ & 1.58 & pass \\
\hline $\mathrm{Z108}$ & 6.2840 & 25.024 & 12.28 & 2.96 & 6.4299 & 3.3014 & 1.10 & $37 \%$ & 1.60 & pass \\
\hline 2109 & 6.2941 & 25.152 & 12.29 & 2.98 & 6.4329 & 3.3024 & 1.10 & $37 \%$ & 1.59 & pass \\
\hline $\mathrm{Z110}$ & 6.2837 & 25.151 & 12.28 & 2.98 & 6.4321 & 3.3024 & 1.10 & $37 \%$ & 1.58 & pass \\
\hline 2111 & 6.2904 & 25.152 & 12.29 & 2.98 & 6.4322 & 3.3024 & 1.10 & $37 \%$ & 1.59 & pass \\
\hline$Z 112$ & 6.2922 & 25.134 & 12.29 & 2.98 & 6.4319 & 3.3024 & 1.10 & $37 \%$ & 1.59 & pass \\
\hline$Z 113$ & 6.2861 & 25.114 & 12.29 & 2.98 & 6.4303 & 3.3014 & 1.10 & $37 \%$ & 1.59 & pass \\
\hline 2114 & 6.2934 & 25.130 & 12.29 & 2.98 & 6.4311 & 3.3024 & 1.10 & $37 \%$ & 1.59 & pass \\
\hline Z115 & 6.2832 & 25.132 & 12.28 & 2.98 & 6.4310 & 3.3024 & 1.10 & $37 \%$ & 1.59 & pass \\
\hline 2116 & 6.2870 & 25.142 & 12.29 & 2.98 & 6.4303 & 3.3014 & 1.10 & $37 \%$ & 1.58 & pass \\
\hline $\mathrm{Z}_{117}$ & 6.3025 & 25.148 & 12.29 & 2.98 & 6.4308 & 3.3014 & 1.10 & $37 \%$ & 1.59 & pass \\
\hline Z118 & 6.2928 & 25.151 & 12.29 & 2.98 & 6.4313 & 3.3024 & 1.10 & $37 \%$ & 1.59 & pass \\
\hline 2119 & 6.2985 & 25.158 & 12.29 & 2.98 & 6.4313 & 3.3024 & 1.10 & $37 \%$ & 1.59 & pass \\
\hline $\mathrm{z} 120$ & 6.3053 & 25.120 & 12.29 & 2.98 & 6.4320 & 3.3024 & 1.10 & $37 \%$ & 1.59 & pass \\
\hline $\mathrm{Z121}$ & 6.2959 & 25.135 & 12.29 & 2.98 & 6.4308 & 3.3014 & 1.10 & $37 \%$ & 1.59 & pass \\
\hline$\frac{1121}{2122}$ & $\frac{0.9539}{6.2843}$ & $\frac{25.135}{25.130}$ & $\frac{12.29}{12.29}$ & $\frac{2.90}{2.98}$ & $\begin{array}{l}0.43009 \\
6.4309\end{array}$ & 3.3014 & 1.10 & $37 \%$ & 1.59 & $\begin{array}{l}\text { pass } \\
\text { pass }\end{array}$ \\
\hline $\mathrm{Z123}$ & 6.2986 & 25.151 & 12.29 & 2.98 & 6.4329 & 3.3024 & 1.10 & $37 \%$ & 1.59 & pass \\
\hline $\mathrm{Z124}$ & 6.2830 & 25.130 & 12.29 & 2.98 & 6.4319 & 3.3024 & 1.10 & $37 \%$ & 1.58 & pass \\
\hline 2125 & 6.3026 & 25.139 & 12.29 & 2.98 & 6.4306 & 3.3014 & 1.10 & $37 \%$ & 1.59 & pass \\
\hline $\mathrm{Z126}$ & 6.2944 & 25.134 & 12.29 & 2.98 & 6.4309 & 3.3014 & 1.10 & $37 \%$ & 1.59 & pass \\
\hline $\mathrm{Z127}$ & 6.2828 & 25.142 & 12.29 & 2.98 & 6.4327 & 3.3024 & 1.10 & $37 \%$ & 1.58 & pass \\
\hline 2128 & 6.2886 & 25.140 & 12.29 & 2.98 & 6.4315 & 3.3024 & 1.10 & $37 \%$ & 1.59 & $\frac{p \text { pass }}{\text { pass }}$ \\
\hline 2129 & 6.2837 & 25.139 & 12.29 & 2.98 & 6.4321 & 3.3024 & 1.10 & $37 \%$ & 1.58 & pass \\
\hline $\mathrm{Z130}$ & 6.2855 & 25.132 & 12.29 & 2.98 & 6.4322 & 3.3024 & 1.10 & $37 \%$ & 1.58 & pass \\
\hline 2131 & 6.2804 & 25.142 & 12.28 & 2.98 & 6.4323 & 3.3024 & 1.10 & $37 \%$ & 1.58 & pass \\
\hline 2132 & 6.3422 & 25.217 & 12.28 & 2.99 & 6.4330 & 3.3024 & 1.10 & $37 \%$ & 1.61 & pass \\
\hline 2133 & 6.2959 & 25.133 & 12.29 & 2.98 & 6.4321 & 3.3024 & 1.10 & $37 \%$ & 1.59 & pass \\
\hline Z134 & 6.2983 & 25.147 & 12.29 & 2.98 & 6.4320 & 3.3024 & 1.10 & $37 \%$ & 1.59 & pass \\
\hline 2135 & 6.2972 & 25.133 & 12.28 & 2.98 & 6.4319 & 3.3024 & 1.10 & $37 \%$ & 1.59 & pass \\
\hline $\mathrm{Z} 136$ & 6.2922 & 25.140 & 12.29 & 2.98 & 6.4304 & 3.3014 & 1.10 & $37 \%$ & 1.59 & pass \\
\hline 2137 & 6.2877 & 25.144 & 12.29 & 2.98 & 6.4325 & 3.3024 & 1.10 & $37 \%$ & 1.58 & pass \\
\hline 2138 & 6.3043 & 25.177 & 12.29 & 2.99 & 6.4316 & 3.3024 & 1.10 & $37 \%$ & 1.59 & pass \\
\hline 2139 & 6.2862 & 25.112 & 12.28 & 2.97 & 6.4312 & 3.3024 & 1.10 & $37 \%$ & 1.59 & pass \\
\hline $\mathrm{Z} 140$ & 6.2964 & 25.161 & 12.29 & 2.98 & 6.4318 & 3.3024 & 1.10 & $37 \%$ & 1.59 & pass \\
\hline $\mathrm{Z141}$ & 6.2819 & 25.132 & 12.28 & 2.98 & 6.4330 & 3.3024 & 1.10 & $37 \%$ & 1.58 & pass \\
\hline 2142 & 6.2926 & 25.114 & 12.29 & 2.98 & 6.4329 & 3.3024 & 1.10 & $37 \%$ & 1.59 & pass \\
\hline $\mathrm{Z143}$ & 6.2894 & 25.148 & 12.29 & 2.98 & 6.4317 & 3.3024 & 1.10 & $37 \%$ & 1.59 & pass \\
\hline 2144 & 6.2958 & 25.156 & 12.28 & 2.98 & 6.4326 & 3.3024 & 1.10 & $37 \%$ & 1.59 & pass \\
\hline 2145 & 6.2905 & 25.134 & 12.29 & 2.98 & 6.4315 & 3.3024 & 1.10 & $37 \%$ & 1.59 & pass \\
\hline $\mathrm{Z} 146$ & 6.3008 & 25.163 & 12.29 & 2.98 & 6.4320 & 3.3024 & 1.1 & $37 \%$ & 1.59 & pass \\
\hline$Z 147$ & 6.3024 & 25.1 & 12.29 & 2.98 & 6.4322 & 3.3024 & 1.10 & $37 \%$ & 1.59 & pass \\
\hline $\mathrm{Z148}$ & 6.2992 & 25.142 & 12.29 & 2.98 & 6.4320 & 3.3024 & 1.10 & $37 \%$ & 1.59 & pass \\
\hline 2149 & 6.2842 & 25.115 & 12.29 & 2.98 & 6.4305 & 3.3014 & 1.10 & $37 \%$ & 1.59 & pass \\
\hline 2150 & 6.2950 & 25.148 & 12.29 & 2.98 & 6.4321 & 3.3024 & 1.10 & $37 \%$ & 1.59 & pass \\
\hline
\end{tabular}

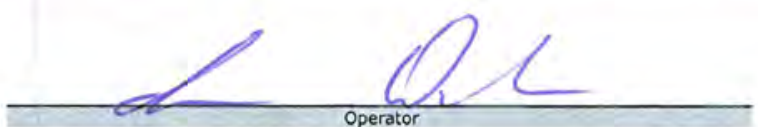

- who 14 acsupervisor

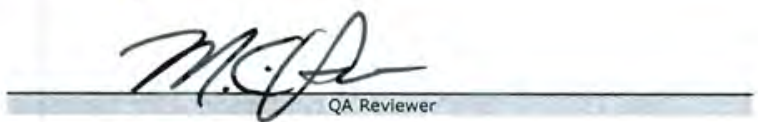

$7-17-09$

$1-11-10$

Date

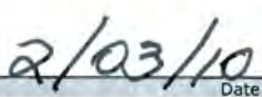


Data Report Form DRF-248: Compact Matrix Density

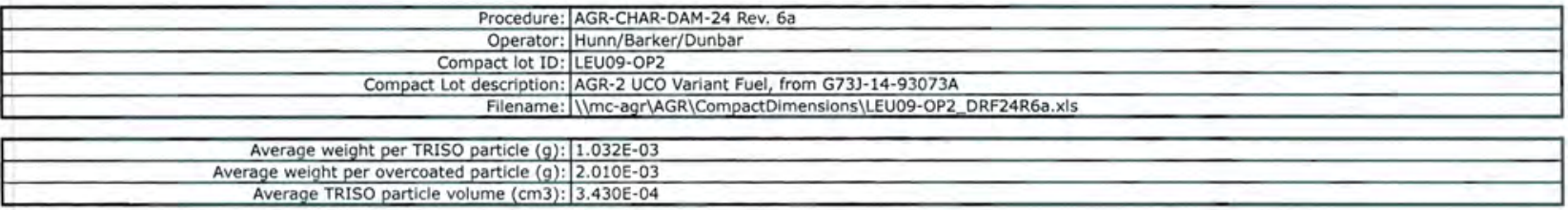

Acceptance criteria for matrix density: $: \geq 1.45$

\begin{tabular}{|c|c|c|c|c|c|c|c|c|c|c|}
\hline $\begin{array}{l}\text { Compact } \\
\text { ID Number }\end{array}$ & $\begin{array}{l}\text { Compact Weight } \\
\text { (a) }\end{array}$ & $\begin{array}{l}\text { Length } \\
(\mathrm{mm})\end{array}$ & $\begin{array}{l}\text { Av. Diameter } \\
(\mathrm{mm})\end{array}$ & $\begin{array}{c}\text { Compact Volume } \\
(\mathrm{cm} 3)\end{array}$ & $\begin{array}{l}\text { Charge Weight } \\
\text { (a) }\end{array}$ & $\begin{array}{l}\text { Particle Weight } \\
\text { (a) }\end{array}$ & $\begin{array}{c}\begin{array}{c}\text { Particle Volume } \\
(\mathrm{cm} 3)\end{array} \\
\end{array}$ & Packing Fraction & $\begin{array}{c}\text { Matrix Density } \\
(\mathrm{a} / \mathrm{cm} 3)\end{array}$ & $\begin{array}{c}\text { Accept? } \\
\text { (pass or fail) }\end{array}$ \\
\hline 2151 & 6.2941 & 25.156 & 12.29 & 2.98 & 6.4315 & 3.3024 & 1.10 & $37 \%$ & 1.59 & pass \\
\hline $\mathrm{Z152}$ & 6.3043 & 25.140 & 12.29 & 2.98 & 6.4319 & 3.3024 & 1.10 & $37 \%$ & 1.59 & pass \\
\hline $\mathrm{Z153}$ & 6.2956 & 25.173 & 12.29 & 2.98 & 6.4305 & 3.3014 & 1.10 & $37 \%$ & 1.59 & pass \\
\hline 2154 & 6.2861 & 25.146 & 12.29 & 2.98 & 6.4311 & 3.3024 & 1.10 & $37 \%$ & 1.58 & pass \\
\hline 2155 & 6.2828 & 25.125 & 12.29 & 2.98 & 6.4305 & 3.3014 & 1.10 & $37 \%$ & 1.58 & pass \\
\hline 2156 & 6.3057 & 25.156 & 12.29 & 2.98 & 6.4330 & 3.3024 & 1.10 & $37 \%$ & 1.59 & pass \\
\hline 2157 & 6.3038 & 25.152 & 12.29 & 2.98 & 6.4315 & 3.3024 & 1.10 & $37 \%$ & 1.59 & pass \\
\hline 2158 & 6.2780 & 25.133 & 12.28 & 2.98 & 6.4313 & 3.3024 & 1.10 & $37 \%$ & 1.58 & pass \\
\hline 2159 & 6.3027 & 25.132 & 12.29 & 2.98 & 6.4321 & 3.3024 & 1.10 & $37 \%$ & 1.59 & pass \\
\hline $\mathrm{Z160}$ & 6.2813 & 25.060 & 12.28 & 2.97 & 6.4320 & 3.3024 & 1.10 & $37 \%$ & 1.59 & pass \\
\hline $\mathrm{Z161}$ & 6.2758 & 25.140 & 12.29 & 2.98 & 6.4308 & 3.3014 & 1.10 & $37 \%$ & 1.58 & pass \\
\hline$Z 162$ & 6.2839 & 25.125 & 12.29 & 2.98 & 6.4323 & 3.3024 & 1.10 & $37 \%$ & 1.59 & pass \\
\hline 2163 & 6.2985 & 25.142 & 12.29 & 2.98 & 6.4321 & 3.3024 & 1.10 & $37 \%$ & 1.59 & pass \\
\hline $\mathrm{Z} 164$ & 6.2829 & 25.048 & 12.29 & 2.97 & 6.4319 & 3.3024 & 1.10 & $37 \%$ & 1.59 & pass \\
\hline 2165 & 6.2897 & 25.116 & 12.28 & 2.97 & 6.4317 & 3.3024 & 1.10 & $37 \%$ & 1.59 & pass \\
\hline $\mathrm{Z166}$ & 6.2864 & 25.161 & 12.29 & 2.98 & 6.4303 & 3.3014 & 1.10 & $37 \%$ & 1.58 & pass \\
\hline 2167 & 6.2828 & 25.168 & 12.29 & 2.98 & 6.4315 & 3.3024 & 1.10 & $37 \%$ & 1.58 & pass \\
\hline $\mathrm{Z168}$ & & & & & & & & & & \\
\hline 2169 & & & & & & & & & & \\
\hline 2170 & & & & & & & & & & \\
\hline 2171 & & & & & & & & & & \\
\hline Z172 & & & & & & & & & & \\
\hline 2173 & & & & & & & & & & \\
\hline 2174 & & & & & & & & & & \\
\hline 2175 & & & & & & & & & & \\
\hline 2176 & & & & & & & & & & \\
\hline 2177 & & & & & & & & & & \\
\hline Z178 & & & & & & & & & & \\
\hline Z179 & & & & & & & & & & \\
\hline $\mathrm{Z} 180$ & & & & & & & & & & \\
\hline $\mathrm{Z181}$ & & & & & & & & & & \\
\hline $\mathrm{Z182}$ & & & & & & & & & & \\
\hline 2183 & & & & & & & & & & \\
\hline $\mathrm{Z184}$ & & & & & & & & & & \\
\hline 2185 & & & & & & & & & & \\
\hline 2186 & & & & & & & & & & \\
\hline 2187 & & & & & & & & & & \\
\hline 2188 & & & & & & & & & & \\
\hline 2189 & & & & & & & & & & \\
\hline Z190 & & & & & & & & & & \\
\hline Z191 & & & & & & & & & & \\
\hline Z192 & & & & & & & & & & \\
\hline 2193 & & & & & & & & & & \\
\hline $\mathrm{Z194}$ & & & & & & & & & & \\
\hline 2195 & & & & & & & & & & \\
\hline Z196 & & & & & & & & & & \\
\hline 2197 & & & & & & & & & & \\
\hline Z198 & & & & & & & & & & \\
\hline Z199 & & & & & & & & & & \\
\hline 2200 & & & & & & & & & & \\
\hline
\end{tabular}


Data Report Form DRF-24C: Compact Tracking

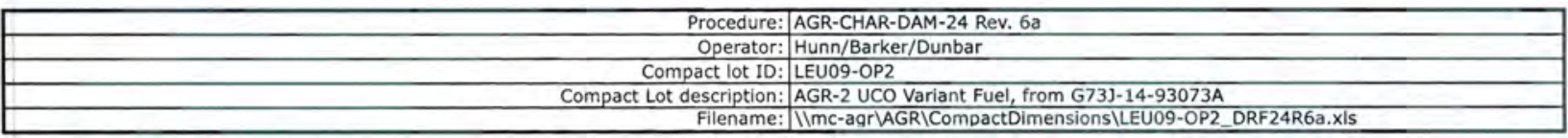

\begin{tabular}{|c|c|}
\hline $\begin{array}{l}\text { Compact } \\
\text { Z Number }\end{array}$ & $\begin{array}{c}\text { Compact G } \\
\text { Number }\end{array}$ \\
\hline$Z 001$ & $\mathrm{G094}$ \\
\hline 2002 & G019 \\
\hline 2003 & G041 \\
\hline$Z 004$ & G087 \\
\hline$Z 005$ & G153 \\
\hline$Z 006$ & G008 \\
\hline Zo07 & G111 \\
\hline$Z 008$ & G039 \\
\hline 2009 & G053 \\
\hline 2010 & G118 \\
\hline Z011 & G096 \\
\hline$Z 012$ & G082 \\
\hline$Z 013$ & G069 \\
\hline Z014 & G026 \\
\hline$Z 015$ & G006 \\
\hline 2016 & G047 \\
\hline Z017 & $\mathrm{G} 048$ \\
\hline$Z 018$ & $\mathrm{G} 046$ \\
\hline 2019 & G061 \\
\hline 2020 & 6080 \\
\hline 2021 & G036 \\
\hline 2022 & G135 \\
\hline 2023 & G085 \\
\hline 2024 & G099 \\
\hline Z025 & G052 \\
\hline 2026 & G109 \\
\hline$Z 027$ & G060 \\
\hline Z028 & G163 \\
\hline 2029 & G114 \\
\hline 2030 & G075 \\
\hline Z031 & G009 \\
\hline Z032 & G027 \\
\hline Z033 & G148 \\
\hline 2034 & G050 \\
\hline 2035 & G015 \\
\hline Z036 & G156 \\
\hline 2037 & G058 \\
\hline 2038 & G044 \\
\hline 2039 & G074 \\
\hline$Z 040$ & G162 \\
\hline 2041 & G185 \\
\hline 2042 & G097 \\
\hline Z043 & G175 \\
\hline$Z 044$ & G176 \\
\hline$Z 045$ & G049 \\
\hline 2046 & G062 \\
\hline 2047 & G142 \\
\hline 2048 & G157 \\
\hline 2049 & G012 \\
\hline Z050 & G145 \\
\hline
\end{tabular}

\begin{tabular}{|c|c|}
\hline $\begin{array}{c}\text { Compact } \\
\text { Z Number }\end{array}$ & $\begin{array}{c}\text { Compact G } \\
\text { Number }\end{array}$ \\
\hline Z051 & G116 \\
\hline Z052 & G083 \\
\hline Z053 & G013 \\
\hline Z054 & G107 \\
\hline Z055 & G121 \\
\hline Z056 & G077 \\
\hline Z057 & G086 \\
\hline Z058 & G138 \\
\hline Z059 & G134 \\
\hline Z060 & G144 \\
\hline Z061 & G167 \\
\hline Z062 & G028 \\
\hline Z063 & G139 \\
\hline Z064 & G057 \\
\hline Z065 & G173 \\
\hline Z066 & G098 \\
\hline Z067 & G120 \\
\hline Z068 & G079 \\
\hline Z069 & G081 \\
\hline Z070 & G035 \\
\hline Z071 & G093 \\
\hline Z072 & G181 \\
\hline Z073 & G143 \\
\hline Z074 & G051 \\
\hline Z075 & G122 \\
\hline Z076 & G178 \\
\hline Z077 & G113 \\
\hline Z078 & G126 \\
\hline Z079 & G070 \\
\hline Z080 & G032 \\
\hline Z081 & G182 \\
\hline Z082 & G154 \\
\hline Z083 & G076 \\
\hline Z084 & G152 \\
\hline Z085 & G011 \\
\hline Z086 & G059 \\
\hline Z087 & G117 \\
\hline Z088 & G124 \\
\hline Z089 & G063 \\
\hline Z090 & G040 \\
\hline Z091 & G140 \\
\hline Z092 & G184 \\
\hline Z093 & G123 \\
\hline Z094 & G055 \\
\hline Z095 & G067 \\
\hline Z096 & G084 \\
\hline G177 & G042 \\
\hline G056 \\
\hline
\end{tabular}

\begin{tabular}{|c|c|}
\hline $\begin{array}{l}\text { Compact } \\
\text { Z Number }\end{array}$ & $\begin{array}{c}\text { Compact G } \\
\text { Number }\end{array}$ \\
\hline Z101 & G179 \\
\hline $\mathrm{Z} 102$ & G158 \\
\hline Z103 & G131 \\
\hline Z104 & G183 \\
\hline Z105 & G092 \\
\hline Z106 & G091 \\
\hline$Z 107$ & G166 \\
\hline Z108 & 6005 \\
\hline Z109 & G141 \\
\hline $\mathrm{Z} 110$ & G129 \\
\hline Z111 & G132 \\
\hline$Z 112$ & G017 \\
\hline $\mathrm{Z} 113$ & G029 \\
\hline$Z 114$ & G164 \\
\hline Z115 & G095 \\
\hline Z116 & G160 \\
\hline Z117 & G165 \\
\hline $\mathrm{Z} 118$ & G168 \\
\hline 2119 & G102 \\
\hline$Z 120$ & G174 \\
\hline$Z 121$ & G103 \\
\hline $\mathrm{Z} 122$ & G030 \\
\hline Z123 & G101 \\
\hline Z124 & G018 \\
\hline Z125 & G110 \\
\hline Z126 & G125 \\
\hline Z127 & G038 \\
\hline Z128 & G151 \\
\hline Z129 & G172 \\
\hline Z130 & G064 \\
\hline Z131 & G025 \\
\hline Z132 & G045 \\
\hline Z133 & G169 \\
\hline 2134 & G106 \\
\hline 2135 & G073 \\
\hline $\mathrm{Z} 136$ & G170 \\
\hline Z137 & G171 \\
\hline Z138 & G078 \\
\hline Z139 & G066 \\
\hline Z140 & G180 \\
\hline Z141 & G136 \\
\hline$Z 142$ & G037 \\
\hline Z143 & G043 \\
\hline Z144 & G071 \\
\hline Z145 & G054 \\
\hline Z146 & G128 \\
\hline Z147 & G112 \\
\hline Z148 & G090 \\
\hline Z149 & G150 \\
\hline $\mathrm{Z} 150$ & G119 \\
\hline
\end{tabular}

\begin{tabular}{|c|c|}
\hline $\begin{array}{l}\text { Compact } \\
\text { Z Number }\end{array}$ & $\begin{array}{c}\text { Compact G } \\
\text { Number }\end{array}$ \\
\hline Z151 & G133 \\
\hline$Z 152$ & G100 \\
\hline $\mathrm{Z153}$ & G108 \\
\hline $\mathrm{Z154}$ & G115 \\
\hline $\mathrm{Z155}$ & G161 \\
\hline$Z 156$ & G072 \\
\hline Z157 & G149 \\
\hline Z158 & G137 \\
\hline Z159 & G127 \\
\hline $\mathrm{Z} 160$ & G004 \\
\hline$Z 161$ & $\mathrm{G} 010$ \\
\hline Z162 & G014 \\
\hline Z163 & G088 \\
\hline Z164 & G001 \\
\hline Z165 & G089 \\
\hline Z166 & G159 \\
\hline $\mathrm{Z} 167$ & G031 \\
\hline$Z 168$ & \\
\hline Z169 & \\
\hline $\mathrm{Z170}$ & \\
\hline Z171 & \\
\hline $\mathrm{Z} 172$ & \\
\hline Z173 & \\
\hline $\mathrm{Z174}$ & \\
\hline $\mathrm{Z} 175$ & \\
\hline$Z 176$ & \\
\hline$Z 177$ & \\
\hline Z178 & \\
\hline 2179 & \\
\hline $\mathrm{Z180}$ & \\
\hline 2181 & \\
\hline$Z 182$ & \\
\hline 2183 & \\
\hline Z184 & \\
\hline$Z 185$ & \\
\hline 2186 & \\
\hline 2187 & \\
\hline Z188 & \\
\hline 2189 & \\
\hline $\mathrm{Z} 190$ & \\
\hline Z191 & \\
\hline Z192 & \\
\hline Z193 & \\
\hline Z194 & \\
\hline Z195 & \\
\hline$Z 196$ & \\
\hline Z197 & \\
\hline$Z 198$ & \\
\hline Z199 & \\
\hline 2200 & \\
\hline
\end{tabular}

\begin{tabular}{|c|c|}
\hline $\begin{array}{l}\text { Compact } \\
\text { Z Number }\end{array}$ & $\begin{array}{l}\text { Compact G } \\
\text { Number }\end{array}$ \\
\hline \multicolumn{2}{|l|}{$\mathrm{Z201}$} \\
\hline \multicolumn{2}{|l|}{2202} \\
\hline \multicolumn{2}{|l|}{2203} \\
\hline \multicolumn{2}{|l|}{ Z204 } \\
\hline \multicolumn{2}{|l|}{2205} \\
\hline \multicolumn{2}{|l|}{ Z206 } \\
\hline \multicolumn{2}{|l|}{ Z207 } \\
\hline \multicolumn{2}{|l|}{$Z 208$} \\
\hline \multicolumn{2}{|l|}{2209} \\
\hline \multicolumn{2}{|l|}{$\frac{2210}{7211}$} \\
\hline \multicolumn{2}{|l|}{2211} \\
\hline \multicolumn{2}{|l|}{2212} \\
\hline \multicolumn{2}{|l|}{2213} \\
\hline \multicolumn{2}{|l|}{2214} \\
\hline \multicolumn{2}{|l|}{$\frac{Z 215}{Z 216}$} \\
\hline \multicolumn{2}{|l|}{2216} \\
\hline \multicolumn{2}{|l|}{ Z217 } \\
\hline \multicolumn{2}{|l|}{$Z 218$} \\
\hline \multicolumn{2}{|l|}{$Z 219$} \\
\hline \multicolumn{2}{|l|}{2220} \\
\hline \multicolumn{2}{|l|}{2221} \\
\hline \multicolumn{2}{|l|}{ Z222 } \\
\hline \multicolumn{2}{|l|}{2223} \\
\hline 2224 & \\
\hline$Z 225$ & \\
\hline Z226 & \\
\hline 2227 & \\
\hline$Z 228$ & \\
\hline$Z 229$ & \\
\hline$Z 230$ & \\
\hline Z231 & \\
\hline$Z 232$ & \\
\hline 2233 & \\
\hline Z234 & \\
\hline Z235 & \\
\hline Z236 & \\
\hline Z237 & \\
\hline$Z 238$ & \\
\hline 2239 & \\
\hline 2240 & \\
\hline 2241 & \\
\hline 2242 & \\
\hline$Z 243$ & \\
\hline 2244 & \\
\hline 2245 & \\
\hline$Z 246$ & \\
\hline$Z 247$ & \\
\hline 2248 & \\
\hline$Z 249$ & \\
\hline 2250 & \\
\hline
\end{tabular}

Comments

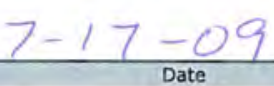


Data Report Form DRF-24D: Compact Charge Weight

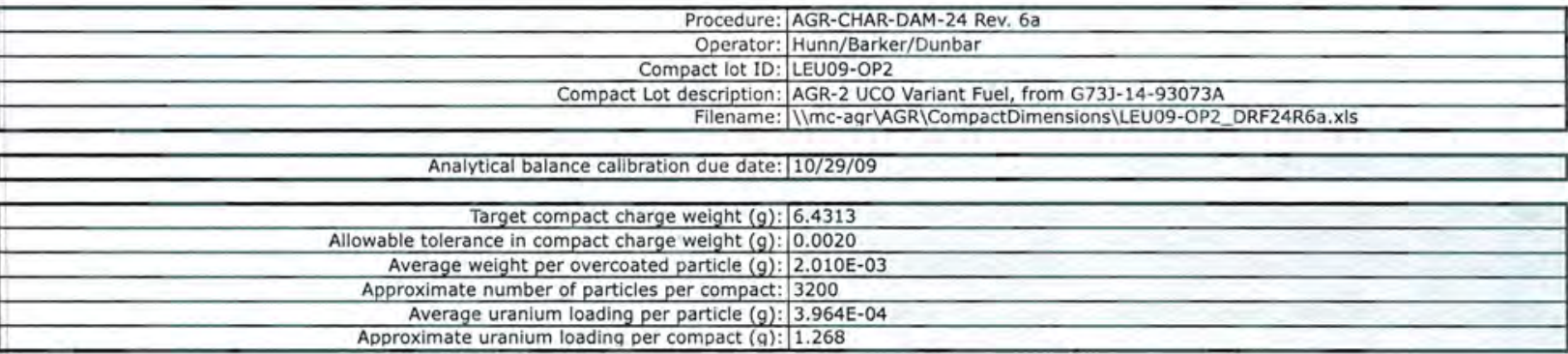

\begin{tabular}{|c|c|}
\hline $\begin{array}{c}\text { Compact } \\
\text { G Number }\end{array}$ & $\begin{array}{c}\text { Charge Weight } \\
\text { (q) }\end{array}$ \\
\hline G001 & 6.4319 \\
\hline G002 & 6.4322 \\
\hline G003 & 6.4309 \\
\hline G004 & 6.4320 \\
\hline G005 & 6.4299 \\
\hline G006 & 6.4325 \\
\hline G007 & 6.4321 \\
\hline G008 & 6.4313 \\
\hline G009 & 6.4321 \\
\hline G010 & 6.4308 \\
\hline G011 & 6.4303 \\
\hline G012 & 6.4333 \\
\hline G013 & 6.4321 \\
\hline G014 & 6.4323 \\
\hline G015 & 6.4310 \\
\hline G016 & 6.4304 \\
\hline G017 & 6.4319 \\
\hline G018 & 6.4319 \\
\hline G019 & 6.4306 \\
\hline G020 & 6.4307 \\
\hline G021 & 6.4300 \\
\hline G022 & 6.4309 \\
\hline G023 & 6.4316 \\
\hline G024 & 6.4303 \\
\hline G025 & 6.4323 \\
\hline G026 & 6.4311 \\
\hline G027 & 6.4308 \\
\hline G028 & 6.4306 \\
\hline G029 & 6.4303 \\
\hline G030 & 6.4309 \\
\hline G031 & 6.4315 \\
\hline G032 & 6.4322 \\
\hline G033 & 6.4307 \\
\hline G034 & 6.4325 \\
\hline G035 & 6.4324 \\
\hline G036 & 6.4323 \\
\hline G037 & 6.4329 \\
\hline G038 & 6.4327 \\
\hline G039 & 6.4318 \\
\hline G040 & 6.4321 \\
\hline G041 & 6.4303 \\
\hline G042 & 6.4316 \\
\hline G043 & 6.4317 \\
\hline G044 & 6.4326 \\
\hline G045 & 6.4330 \\
\hline G046 & 6.4329 \\
\hline G048 & 6.4315 \\
\hline & 6.4322 \\
\hline & 6.4325 \\
\hline
\end{tabular}

\begin{tabular}{|c|c|}
\hline $\begin{array}{c}\text { Compact } \\
\text { G Number }\end{array}$ & $\begin{array}{c}\text { Charge Weight } \\
(\mathrm{q})\end{array}$ \\
\hline G051 & 6.4308 \\
\hline G052 & 6.4302 \\
\hline G053 & 6.4326 \\
\hline G054 & 6.4315 \\
\hline G055 & 6.4314 \\
\hline G056 & 6.4321 \\
\hline G057 & 6.4314 \\
\hline G058 & 6.4309 \\
\hline G059 & 6.4322 \\
\hline G060 & 6.4320 \\
\hline G061 & 6.4325 \\
\hline G062 & 6.4311 \\
\hline G063 & 6.4304 \\
\hline G064 & 6.4322 \\
\hline G065 & 6.4319 \\
\hline G066 & 6.4312 \\
\hline G067 & 6.4306 \\
\hline G068 & 6.4308 \\
\hline G069 & 6.4313 \\
\hline G070 & 6.4323 \\
\hline G071 & 6.4326 \\
\hline G072 & 6.4330 \\
\hline G073 & 6.4319 \\
\hline G074 & 6.4310 \\
\hline G075 & 6.4304 \\
\hline G076 & 6.4302 \\
\hline G077 & 6.4316 \\
\hline G078 & 6.4316 \\
\hline G079 & 6.4325 \\
\hline G080 & 6.4312 \\
\hline G081 & 6.4325 \\
\hline G082 & 6.4329 \\
\hline G083 & 6.4315 \\
\hline G084 & 6.4322 \\
\hline G085 & 6.4314 \\
\hline G086 & 6.4309 \\
\hline G087 & 6.4318 \\
\hline G088 & 6.4321 \\
\hline G089 & 6.4317 \\
\hline G090 & 6.4320 \\
\hline G091 & 6.4314 \\
\hline G092 & 6.4313 \\
\hline G093 & 6.4326 \\
\hline G094 & 6.4327 \\
\hline G095 & 6.4310 \\
\hline G096 & 6.4307 \\
\hline G097 & 6.4329 \\
\hline G098 & 6.4300 \\
\hline G100 & 6.4302 \\
\hline 6.4319 \\
\hline
\end{tabular}

\begin{tabular}{|c|c|}
\hline $\begin{array}{c}\text { Compact } \\
\text { G Number }\end{array}$ & $\begin{array}{c}\text { Charge Weight } \\
\text { (a) }\end{array}$ \\
\hline G101 & 6.4329 \\
\hline G102 & 6.4313 \\
\hline G103 & 6.4308 \\
\hline G104 & 6.4311 \\
\hline G105 & 6.4326 \\
\hline G106 & 6.4320 \\
\hline G107 & 6.4316 \\
\hline G108 & 6.4305 \\
\hline G109 & 6.4314 \\
\hline G110 & 6.4306 \\
\hline G111 & 6.4311 \\
\hline G112 & 6.4322 \\
\hline G113 & 6.4319 \\
\hline G114 & 6.4328 \\
\hline G115 & 6.4311 \\
\hline G116 & 6.4303 \\
\hline G117 & 6.4328 \\
\hline G118 & 6.4322 \\
\hline G119 & 6.4321 \\
\hline G120 & 6.4311 \\
\hline G121 & 6.4321 \\
\hline G122 & 6.4309 \\
\hline G123 & 6.4310 \\
\hline G124 & 6.4320 \\
\hline G125 & 6.4309 \\
\hline G126 & 6.4323 \\
\hline G127 & 6.4321 \\
\hline G128 & 6.4320 \\
\hline G129 & 6.4321 \\
\hline G130 & 6.4314 \\
\hline G131 & 6.4306 \\
\hline G132 & 6.4322 \\
\hline G133 & 6.4315 \\
\hline G134 & 6.4307 \\
\hline G135 & 6.4311 \\
\hline G136 & 6.4330 \\
\hline G137 & 6.4313 \\
\hline G138 & 6.4295 \\
\hline G139 & 6.4314 \\
\hline G140 & 6.4302 \\
\hline G141 & 6.4329 \\
\hline G142 & 6.4316 \\
\hline G143 & 6.4311 \\
\hline G144 & 6.4308 \\
\hline G145 & 6.4318 \\
\hline G146 & 6.4301 \\
\hline G147 148 & 6.4325 \\
\hline G150 & 6.4326 \\
\hline & 6.4315 \\
\hline & 6.4305 \\
\hline
\end{tabular}

\begin{tabular}{|c|c|}
\hline $\begin{array}{c}\text { Compact } \\
\text { G Number }\end{array}$ & $\begin{array}{c}\text { Charge Weight } \\
\text { (a) }\end{array}$ \\
\hline G151 & 6.4315 \\
\hline G152 & 6.4325 \\
\hline G153 & 6.4309 \\
\hline G154 & 6.4313 \\
\hline G155 & 6.4311 \\
\hline G156 & 6.4326 \\
\hline G157 & 6.4306 \\
\hline G158 & 6.4319 \\
\hline G159 & 6.4303 \\
\hline G160 & 6.4303 \\
\hline G161 & 6.4305 \\
\hline G162 & 6.4327 \\
\hline G163 & 6.4320 \\
\hline G164 & 6.4311 \\
\hline G165 & 6.4308 \\
\hline G167 & 6.4299 \\
\hline G168 & 6.4323 \\
\hline G169 & 6.4313 \\
\hline G170 & 6.4321 \\
\hline G171 & 6.4304 \\
\hline G172 & 6.4325 \\
\hline G173 & 6.4321 \\
\hline G174 & 6.4321 \\
\hline G175 & 6.4320 \\
\hline G176 & 6.4309 \\
\hline G177 & 6.4321 \\
\hline G178 & 6.4331 \\
\hline G179 & 6.4327 \\
\hline G180 & 6.4318 \\
\hline G181 & 6.4323 \\
\hline G182 & 6.4314 \\
\hline G183 & 6.4327 \\
\hline G184 & 6.4317 \\
\hline G185 & 6.4304 \\
\hline G186 & \\
\hline G187 & \\
\hline G188 & \\
\hline G189 & \\
\hline G190 & \\
\hline G191 & \\
\hline G192 & \\
\hline G193 & \\
\hline G194 & \\
\hline G196 & \\
\hline G198 & \\
\hline & \\
\hline & \\
\hline & \\
\hline & \\
\hline & \\
\hline & \\
\hline & \\
\hline & \\
\hline & \\
\hline
\end{tabular}

\begin{tabular}{|c|c|}
\hline $\begin{array}{l}\text { Compact } \\
\text { G Number }\end{array}$ & $\begin{array}{c}\text { Charge } \\
\text { Weight (q) }\end{array}$ \\
\hline G201 & \\
\hline G202 & \\
\hline G203 & \\
\hline G204 & \\
\hline G205 & \\
\hline G206 & 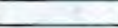 \\
\hline G207 & \\
\hline G208 & \\
\hline G209 & \\
\hline G210 & \\
\hline G211 & \\
\hline G212 & \\
\hline G213 & \\
\hline G214 & \\
\hline G215 & \\
\hline G216 & \\
\hline G217 & \\
\hline G218 & \\
\hline G219 & \\
\hline G220 & \\
\hline $\mathrm{G} 221$ & \\
\hline G222 & \\
\hline G223 & \\
\hline G224 & \\
\hline G225 & \\
\hline G226 & \\
\hline G227 & \\
\hline G228 & \\
\hline G229 & \\
\hline G230 & \\
\hline G231 & \\
\hline G232 & \\
\hline G233 & \\
\hline G234 & \\
\hline G235 & \\
\hline G236 & \\
\hline G237 & \\
\hline G238 & \\
\hline G239 & \\
\hline G240 & \\
\hline $\mathrm{G} 241$ & \\
\hline G242 & \\
\hline G243 & \\
\hline G244 & \\
\hline G245 & \\
\hline G246 & \\
\hline G247 & \\
\hline G248 & \\
\hline G249 & \\
\hline G250 & \\
\hline
\end{tabular}

Comments

Average weight per overcoated particle from combined results of 2 independent measurements (W09060801 and W09060802).

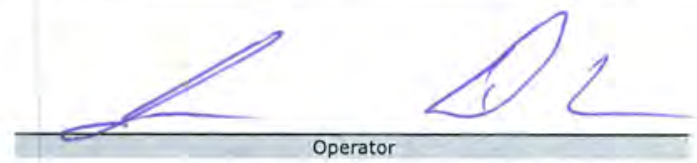

$$
6-23-0 \%
$$


Data Report Form DRF-24A: Compact Diameter and Length

Procedure: AGR-CHAR-DAM-24 Rev. 6a

Operator: Dunbar, Barker, Hunn, West

Compact lot ID: LEU11-OP2-Z

Compact Lot description: AGR-2 B\&W UO2 Fuel, from G73H-10-93085

Filename: IImc-arr|AGRICompactDimensions ILEU11-OP2 DRF24R6a.XIs

\begin{tabular}{|c|l|}
\hline Vertical height gauge calibration due date: & $3 / 6 / 10$ \\
\hline Pass-thru block calibration due date: & $1 / 17 / 11$ \\
\hline Digital callper calibration due date: & $7 / 7 / 10$ \\
\hline Gauge blocks calibration due date: & $11 / 7 / 12$ \\
\hline Analytical balance callibration due date: & $2 / 12 / 10$ \\
\hline
\end{tabular}

\begin{tabular}{|r|l|}
\hline Acceptance criteria for compact length: & $\geq 25.02$ and $\leq 25.40 \mathrm{~mm}$ \\
\hline Acceptance criteria for compact diameter: & $\geq 12.22$ and $\leq 12.46 \mathrm{~mm}$ (and pass through $12.46 \mathrm{~mm}$ ring gauge) \\
\hline Acceptance criteria for compact mass: & For information only \\
\hline
\end{tabular}

\begin{tabular}{|c|c|c|c|c|c|c|c|c|c|c|}
\hline \multirow{2}{*}{$\begin{array}{c}\text { Compact } \\
\text { ID Number }\end{array}$} & \multirow{2}{*}{$\begin{array}{l}\text { Length } \\
(\mathrm{mm})\end{array}$} & \multicolumn{6}{|c|}{ Diameter $(\mathrm{mm})$} & \multirow{2}{*}{$\begin{array}{l}\text { Pass Thru? } \\
\text { (Yor } \mathrm{N})\end{array}$} & \multirow{2}{*}{$\begin{array}{l}\text { Compact weight } \\
\text { (a) }\end{array}$} & \multirow{2}{*}{$\begin{array}{c}\text { Accept? } \\
\text { (pass or fail) }\end{array}$} \\
\hline & & Top 1 & Top 2 & Middle 1 & Middle 2 & Bottom 1 & Bottom 2 & & & \\
\hline 2001 & 25.143 & 12.27 & 12.27 & 12.28 & 12.28 & 12.27 & 12.27 & $y$ & 6.0964 & pass \\
\hline 2002 & 25.157 & 12.27 & 12.27 & 12.27 & 12.27 & 12.27 & 12.27 & $y$ & 6.1062 & pass \\
\hline 2003 & 25.120 & 12.28 & 12.28 & 12.27 & 12.29 & 12.28 & 12.28 & $y$ & 6.1085 & pass \\
\hline 2004 & 25.152 & 12.26 & 12.27 & 12.27 & 12.26 & 12.27 & 12.26 & $y$ & 6.1391 & pass \\
\hline 2005 & 25.147 & 12.27 & 12.27 & 12.27 & 12.27 & 12.27 & 12.27 & $y$ & 6.1015 & pass \\
\hline 2006 & 25.102 & 12.26 & 12.26 & 12.27 & 12.27 & 12.27 & 12.26 & $\frac{y}{y}$ & 6.0972 & pass \\
\hline 2007 & 25.079 & 12.26 & 12.26 & 12.27 & 12.28 & 12.27 & 12.27 & $y$ & 6.0897 & pass \\
\hline 2008 & 25.092 & 12.27 & 12.27 & 12.27 & 12.28 & 12.27 & 12.28 & $y$ & 6.0936 & pass \\
\hline 2009 & 25.142 & 12.27 & 12.27 & 12.27 & 12.27 & 12.27 & 12.27 & $y$ & 6.1066 & pass \\
\hline 2010 & 25.135 & 12.27 & 12.27 & 12.28 & 12.27 & 12.27 & 12.27 & $\frac{y}{y}$ & 6.0934 & pass \\
\hline 2011 & 25.123 & 12.28 & 12.27 & 12.27 & 12.27 & 12.27 & 12.27 & $\frac{y}{y}$ & 6.0987 & pass \\
\hline $\mathrm{Z} 012$ & 25.142 & 12.26 & 12.26 & 12.26 & 12.26 & 12.27 & 12.27 & $\frac{y}{y}$ & 6.1013 & pass \\
\hline 2013 & 25.125 & 12.28 & 12.28 & 12.28 & 12.27 & 12.27 & 12.27 & $\frac{1}{y}$ & 6.0958 & pass \\
\hline 2014 & 25.126 & 12.28 & 12.28 & 12.28 & 12.28 & 12.28 & 12.27 & $y$ & 6.0791 & pass \\
\hline 2015 & 25.163 & 12.27 & 12.28 & 12.27 & 12.27 & 12.27 & 12.27 & $y$ & 6.0893 & pass \\
\hline 2016 & 25.140 & 12.26 & 12.26 & 12.27 & 12.27 & 12.27 & 12.27 & $y$ & 6.0936 & pass \\
\hline 2017 & 25.121 & 12.27 & 12.27 & 12.27 & 12.28 & 12.28 & 12.28 & $y$ & 6.0878 & pass \\
\hline 2018 & 25.121 & 12.28 & 12.28 & 12.28 & 12.29 & 12.28 & 12.28 & $y$ & 6.0958 & pass \\
\hline 2019 & 25.140 & 12.27 & 12.27 & 12.27 & 12.27 & 12.27 & 12.27 & $y$ & 6.1200 & pass \\
\hline 2020 & 25.126 & 12.28 & 12.27 & 12.27 & 12.27 & 12.28 & 12.27 & $y$ & 6.1045 & pass \\
\hline 2021 & 25.146 & 12.27 & 12.27 & 12.27 & 12.27 & 12.28 & 12.27 & $y$ & 6.0851 & pass \\
\hline $\mathrm{Z} 022$ & 25.142 & 12.27 & 12.27 & 12.27 & 12.27 & 12.27 & 12.27 & $\frac{y}{y}$ & 6.0883 & pass \\
\hline 2023 & 25.129 & 12.27 & 12.26 & 12.27 & 12.27 & 12.28 & 12.27 & $y$ & 6.0959 & pass \\
\hline 2024 & 25.101 & 12.28 & 12.28 & 12.28 & 12.28 & 12.27 & 12.27 & $\frac{1}{y}$ & 6.0929 & pass \\
\hline 2025 & 25.135 & 12.27 & 12.27 & 12.27 & 12.27 & 12.27 & 12.27 & $y$ & 6.0873 & pass \\
\hline 2026 & 25.123 & 12.27 & 12.28 & 12.28 & 12.28 & 12.27 & 12.28 & $y$ & 6.0895 & pass \\
\hline 2027 & 25.123 & 12.28 & 12.28 & 12.28 & 12.27 & 12.27 & 12.27 & $y$ & 6.0960 & pass \\
\hline 2028 & 25.162 & 12.27 & 12.27 & 12.27 & 12.27 & 12.27 & 12.27 & $y$ & 6.1439 & pass \\
\hline 2029 & 25.147 & 12.26 & 12.27 & 12.27 & 12.27 & 12.27 & 12.27 & $\frac{1}{y}$ & 6.1439 & pass \\
\hline 2030 & 25.142 & 12.28 & 12.28 & 12.28 & 12.28 & 12.28 & 12.28 & $y$ & 6.0887 & pass \\
\hline Z031 & 25.126 & 12.27 & 12.26 & 12.27 & 12.27 & 12.27 & 12.26 & $y$ & 6.1289 & pass \\
\hline 2032 & 25.124 & 12.26 & 12.26 & 12.27 & 12.27 & 12.27 & 12.27 & $y$ & 6.0971 & pass \\
\hline 2033 & 25.132 & 12.26 & 12.26 & 12.27 & 12.27 & 12.26 & 12.27 & $\frac{y}{y}$ & 6.0935 & pass \\
\hline 2034 & 25.130 & 12.27 & 12.27 & 12.27 & 12.27 & 12.27 & 12.27 & $y$ & 6.0940 & pass \\
\hline 2035 & 25.166 & 12.26 & 12.26 & 12.27 & 12.27 & 12.27 & 12.27 & $y$ & 6.1329 & pass \\
\hline 2036 & 25.132 & 12.26 & 12.27 & 12.27 & 12.27 & 12.27 & 12.28 & $y$ & 6.0949 & pass \\
\hline 2037 & 25.146 & 12.27 & 12.27 & 12.27 & 12.27 & 12.27 & 12.27 & $y$ & 6.0980 & pass \\
\hline 2038 & 25.153 & 12.27 & 12.28 & 12.27 & 12.27 & 12.27 & 12.28 & $y$ & 6.0903 & pass \\
\hline 2039 & 25.133 & 12.28 & 12.27 & 12.27 & 12.28 & 12.28 & 12.28 & $y$ & 6.0786 & pass \\
\hline 2040 & 25.124 & 12.26 & 12.26 & 12.27 & 12.27 & 12.27 & 12.27 & $y$ & 6.1006 & pass \\
\hline Z041 & 25,148 & 12.27 & 12.27 & 12.28 & 12.28 & 12.28 & 12.27 & $y$ & 6.0920 & pass \\
\hline 2042 & 25.123 & 12.26 & 12.26 & 12.26 & 12.27 & 12.27 & 12.27 & $y$ & 6.0867 & pass \\
\hline Z043 & 25.143 & 12.28 & 12.27 & 12.28 & 12.28 & 12.27 & 12.28 & $y$ & 6.0907 & pass \\
\hline 2044 & 25.135 & 12.27 & 12.27 & 12.27 & 12.27 & 12.27 & 12.27 & $y$ & 6.0981 & pass \\
\hline 2045 & 25.148 & 12.28 & 12.28 & 12.28 & 12.28 & 12.28 & 12.27 & $\frac{1}{y}$ & 6.1382 & pass \\
\hline Z046 & 25.110 & 12.28 & 12.28 & 12.28 & 12.27 & 12.27 & 12.27 & $\frac{y}{y}$ & 6.0734 & pass \\
\hline 2047 & 25.128 & 12.27 & 12.27 & 12.27 & 12.27 & 12.28 & 12.27 & $y$ & 6.0965 & pass \\
\hline 2048 & 25.110 & 12.28 & 12.28 & 12.28 & 12.28 & 12.27 & 12.27 & $y$ & 6.1145 & pass \\
\hline Z049 & 25.079 & 12.27 & 12.27 & 12.28 & 12.27 & 12.27 & 12.27 & $y$ & 6.0772 & pass \\
\hline 2050 & 25.168 & 12.25 & 12.26 & 12.25 & 12.26 & 12.26 & 12.26 & $y$ & 6.1225 & pass \\
\hline
\end{tabular}

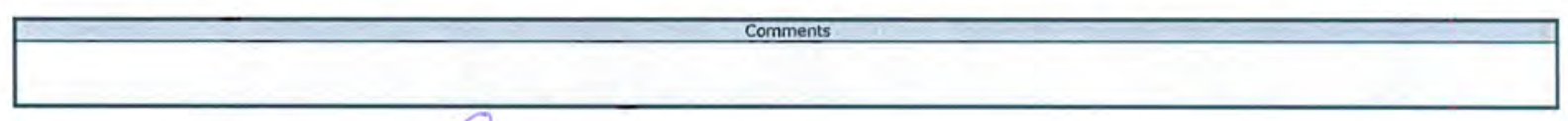

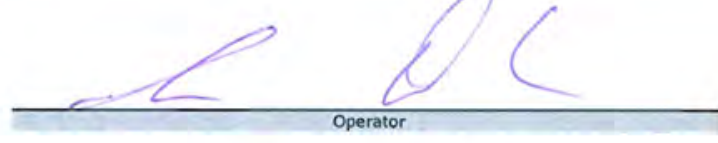
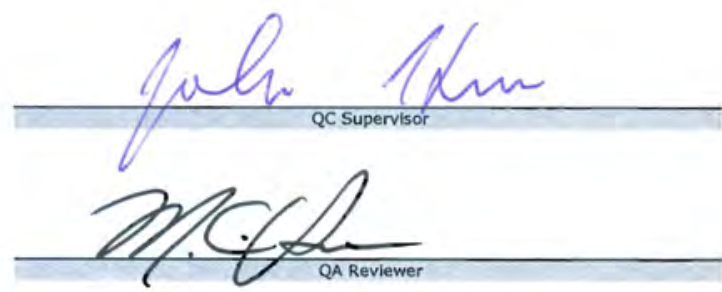

$9-14-09$

$1-12-10$

$2 / 03 / 10$ 
ORNL/TM-2010/296

Data Report Form DRF-24A: Compact Diameter and Length

Procedure: AGR-CHAR-DAM-24 Rev, wa Compact lot ID: LEU11-OP2-Z

Compact Lot description: AGR-2 B\&W UO2 Fuel, from G73H-10-93085B

Filename:| IImc-agr|AGRICompactDimensionsILEU11-OP2 DRF24R6a.xIs

Vertical height gauge calibration due date: $3 / 6 / 10$

Pass-thru block calibration due date: $1 / 17 / 11$

Digital caliper calibration due date: $7 / 7 / 10$

Analytical balance calibration due date: $: 2 / 12 / 10$

Acceptance criteria for compact length: $\geq 25.02$ and $\leq 25.40 \mathrm{~mm}$

Acceptance criteria for compact diameter: $\geq 12.22$ and $\leq 12.46 \mathrm{~mm}$ (and pass through $12.46 \mathrm{~mm}$ ring gauge)

Acceptance criteria for compact mass: For information only

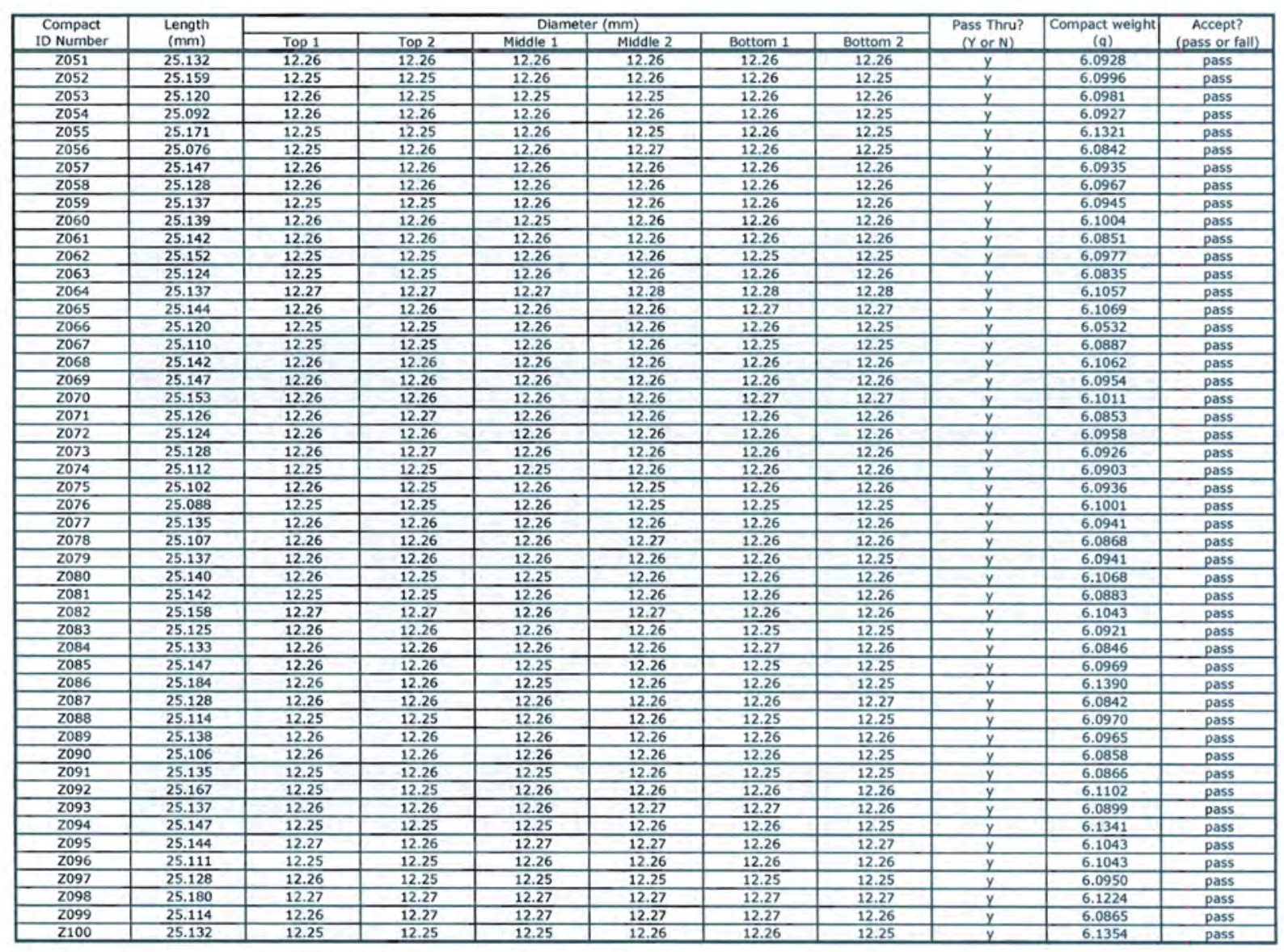
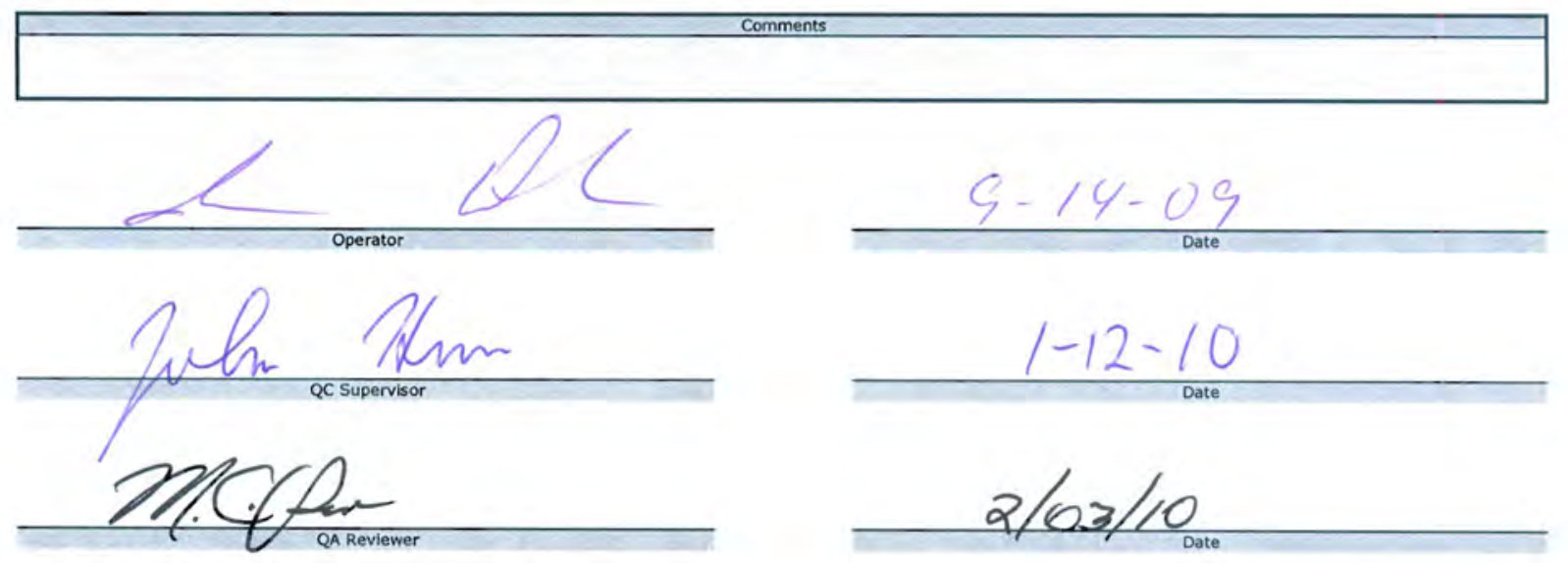

46 
Data Report Form DRE-24A; Compact Dlameter and Lenoth Procedure: AGR-CHAR-DAM-24 Rev. 6a

Operator: Dunbar, Barker

Compact Lot description: $A$ AGR-2 B\&W UO2 Fuel, from G73H-10-93085

Filename: IImc-aorVAGRICOmpactDimensionsILEU11-OP2 DRF24R6a.XI

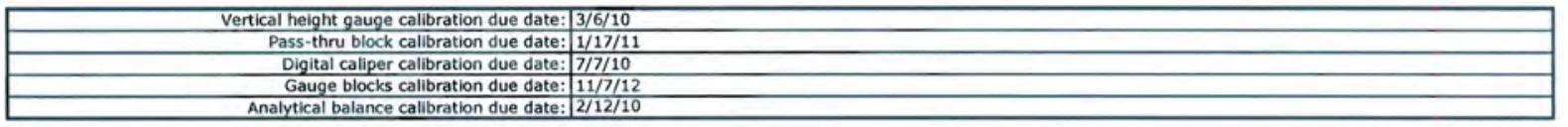

\begin{tabular}{|c|l|}
\hline Acceptance criteria for compact length: & $\geq 25.02$ and $\leq 25.40 \mathrm{~mm}$ \\
\hline Acceptance criteria for compact dlameter: & $\geq 12.22$ and $512.46 \mathrm{~mm}$ (and pass through $12.46 \mathrm{~mm}$ ring gauge) \\
\hline Acceptance criteria for compact mass: & For information only \\
\hline
\end{tabular}

\begin{tabular}{|c|c|c|c|c|c|c|c|c|c|c|}
\hline Compact & Length & \multicolumn{6}{|c|}{ Diameter $(\mathrm{mm})$} & \multirow{2}{*}{$\begin{array}{l}\text { Pass Thru? } \\
(Y \text { or } N)\end{array}$} & \multirow{2}{*}{$\begin{array}{l}\text { Compact weight } \\
\text { (a) }\end{array}$} & \multirow{2}{*}{$\begin{array}{c}\text { Accept? } \\
\text { (pass or fall) }\end{array}$} \\
\hline ID Number & $(\mathrm{mm})$ & Top 1 & Top 2 & Middle 1 & Middle 2 & Bottom 1 & Bottom 2 & & & \\
\hline $\mathrm{Z} 101$ & 25.100 & 12.26 & 12.26 & 12.26 & 12.26 & 12.26 & 12.26 & & & \\
\hline $\mathrm{Z102}$ & 25.095 & 12.25 & 12.25 & 12.25 & 12.25 & 12.25 & 12.25 & $\frac{1}{y}$ & 6.0919 & pass \\
\hline 2103 & 25.114 & 12.26 & 12.27 & 12.27 & 12.28 & 12.27 & 12.27 & $\frac{1}{y}$ & 6.1294 & pass \\
\hline $\mathrm{Z104}$ & 25.142 & 12.26 & 12.26 & 12.27 & 12.27 & 12.27 & 12.27 & $\frac{1}{y}$ & 6.1022 & pass \\
\hline $\mathrm{Z} 105$ & 25.138 & 12.27 & 12.28 & 12.27 & 12.27 & 12.28 & 12.28 & $\frac{1}{y}$ & 6.0773 & pass \\
\hline 2106 & 25.147 & 12.27 & 12.27 & 12.26 & 12.27 & 12.26 & 12.27 & $y$ & 6.1068 & pass \\
\hline $\mathrm{Z107}$ & 25.135 & 12.26 & 12.25 & 12.26 & 12.26 & 12.26 & 12.26 & $\frac{1}{y}$ & 6.0866 & pass \\
\hline $\mathrm{Z108}$ & 25.109 & 12.26 & 12.26 & 12.26 & 12.27 & 12.26 & 12.26 & $y$ & 6.1399 & pass \\
\hline 2109 & 25.123 & 12.26 & 12.26 & 12.26 & 12.26 & 12.26 & 12.26 & $y$ & 6.0896 & pass \\
\hline 2110 & 25.126 & 12.25 & 12.25 & 12.26 & 12.25 & 12.26 & 12.25 & $y$ & 6.0995 & pass \\
\hline Z111 & 25.151 & 12.27 & 12.27 & 12.27 & 12.28 & 12.27 & 12.27 & $\frac{y}{y}$ & 6.1015 & pass \\
\hline 2112 & 25.099 & 12.27 & 12.27 & 12.26 & 12.26 & 12.27 & 12.27 & $\frac{y}{y}$ & 6.1339 & pass \\
\hline 2113 & 25.123 & 12.26 & 12.26 & 12.26 & 12.26 & 12.26 & 12.26 & $y$ & 6.0856 & pass \\
\hline $\mathrm{Z114}$ & 25.144 & 12.27 & 12.27 & 12.28 & 12.27 & 12.27 & 12.26 & $y$ & 6.0762 & pass \\
\hline Z115 & 25.167 & 12.27 & 12.27 & 12.26 & 12.27 & 12.26 & 12.26 & $y$ & 6.1022 & pass \\
\hline$\frac{2116}{717}$ & 25.147 & 12.26 & 12.27 & 12.26 & 12.27 & 12.27 & 12.26 & $y$ & 6.0924 & pass \\
\hline Z117 & 25.142 & 12.26 & 12.27 & 12.27 & 12.26 & 12.27 & 12.27 & $y$ & 6.0856 & pass \\
\hline$\frac{z 118}{710}$ & 25.123 & 12.26 & 12.26 & 12.26 & 12.26 & 12.26 & 12.26 & $y$ & 6.0896 & pass \\
\hline$\frac{Z 119}{7120}$ & 25.099 & 12.27 & $\frac{12.27}{1.26}$ & $\frac{12.28}{1.27}$ & $\frac{12.27}{12.27}$ & 12.27 & 12.27 & $y$ & 6.0741 & pass \\
\hline$\frac{2120}{7121}$ & $\frac{25.096}{25.143}$ & $\frac{12.27}{12.26}$ & $\frac{12.26}{12.26}$ & $\frac{12.27}{12.26}$ & $\frac{12.27}{12.27}$ & $\frac{12.26}{12.26}$ & $\begin{array}{l}12.26 \\
12.25\end{array}$ & $y$ & 6.0703 & pass \\
\hline $\mathrm{z} 121$ & $\frac{25.143}{25.171}$ & $\frac{12.20}{12.26}$ & 12.26 & $\frac{12.26}{1.26}$ & $\frac{12.27}{12.26}$ & $\begin{array}{l}12.26 \\
12.25\end{array}$ & $\frac{12.25}{12.26}$ & $\frac{y}{y}$ & $\frac{6.0950}{6.1342}$ & pass \\
\hline $\mathrm{Z123}$ & 25.129 & 12.26 & 12.26 & 12.26 & 12.26 & 12.26 & 12.27 & $\frac{y}{y}$ & 6.0978 & pass \\
\hline $\mathrm{Z124}$ & 25.125 & 12.27 & 12.27 & 12.27 & 12.27 & 12.27 & 12.27 & $\frac{y}{y}$ & 6.1039 & $\begin{array}{l}\text { pass } \\
\text { pass }\end{array}$ \\
\hline 2125 & 25.133 & 12.26 & 12.27 & 12.27 & 12.27 & 12.26 & 12.27 & $\frac{1}{y}$ & 6.0853 & pass \\
\hline Z126 & 25.132 & 12.25 & 12.25 & 12.25 & 12.26 & 12.26 & 12.26 & $y$ & 6.1411 & pass \\
\hline $\mathrm{Z127}$ & 25.137 & 12.27 & 12.27 & 12.27 & 12.27 & 12.27 & 12.27 & $\frac{1}{y}$ & 6.1158 & pass \\
\hline 2128 & 25.140 & 12.26 & 12.26 & 12.27 & 12.26 & 12.26 & 12.25 & $\frac{1}{y}$ & 6.0947 & pass \\
\hline 2129 & 25.143 & 12.27 & 12.27 & 12.27 & 12.27 & 12.28 & 12.28 & $\frac{1}{y}$ & 6.1264 & pass \\
\hline Z130 & 25.149 & 12.28 & 12.28 & 12.27 & 12.27 & 12.27 & 12.27 & $y$ & 6.1025 & pass \\
\hline $\mathrm{Z131}$ & 25.109 & 12.26 & 12.27 & 12.28 & 12.27 & 12.28 & 12.27 & $\frac{1}{y}$ & 6.0870 & pass \\
\hline $\mathrm{Z132}$ & 25.162 & 12.27 & 12.27 & 12.27 & 12.27 & 12.27 & 12.28 & $\frac{1}{y}$ & 6.1354 & pass \\
\hline 2133 & 25.111 & 12.27 & 12.27 & 12.28 & 12.28 & 12.28 & 12.27 & $\frac{1}{y}$ & 6.0952 & pass \\
\hline 2134 & 25.146 & 12.28 & 12.28 & 12.28 & 12.28 & 12.28 & 12.28 & $\frac{1}{y}$ & 6.0790 & pass \\
\hline 2135 & 25.128 & 12.26 & 12.26 & 12.26 & 12.26 & 12.25 & 12.26 & $\frac{1}{y}$ & 6.1198 & pass \\
\hline 2136 & 25.157 & 12.28 & 12.27 & 12.28 & 12.27 & 12.28 & 12.28 & $y$ & 6.0966 & pass \\
\hline 2137 & 25.149 & 12.28 & 12.27 & 12.28 & 12.28 & 12.28 & 12.29 & $y$ & 6.1086 & pass \\
\hline 2138 & 25.110 & 12.28 & 12.28 & 12.28 & 12.28 & 12.27 & 12.28 & $y$ & 6.0970 & pass \\
\hline 2139 & 25.101 & 12.27 & 12.28 & 12.28 & 12.28 & 12.27 & 12.27 & $y$ & 6.0918 & pass \\
\hline $\mathrm{Z} 140$ & 25.144 & 12.27 & 12.27 & 12.27 & 12.28 & 12.28 & 12.28 & $y$ & 6.1372 & pass \\
\hline$Z 141$ & 25.125 & 12.28 & 12.29 & 12.28 & 12.28 & 12.28 & 12.28 & $y$ & 6.0982 & pass \\
\hline $\mathrm{Z142}$ & 25.146 & 12.28 & 12.29 & 12.29 & 12.29 & 12.29 & 12.28 & $y$ & 6.0985 & pass \\
\hline Z143 & 25.201 & 12.28 & 12.28 & 12.29 & 12.29 & 12.28 & 12.28 & $y$ & 6.1088 & pass \\
\hline Z144 & 25.151 & 12.27 & 12.27 & 12.27 & 12.27 & 12.27 & 12.28 & $y$ & 6.0896 & pass \\
\hline Z145 & 25.161 & 12.26 & 12.26 & 12.26 & 12.26 & 12.26 & 12.26 & $y$ & 6.0878 & pass \\
\hline Z146 & 25.128 & 12.27 & 12.28 & 12.27 & 12.27 & 12.27 & 12.27 & $y$ & 6.0920 & pass \\
\hline$z_{147}$ & 25.100 & 12.28 & 12.28 & 12.28 & 12.28 & 12.28 & 12.28 & $y$ & 6.1164 & pass \\
\hline Z148 & $\begin{array}{r}25.133 \\
25.132\end{array}$ & $\frac{12.28}{12.27}$ & $\frac{12.28}{1.27}$ & $\frac{12.27}{127}$ & $\frac{12.27}{12.27}$ & & 12.27 & y & 6.1121 & pass \\
\hline$\frac{\mathrm{Z} 149}{\mathrm{Z} 150}$ & $\begin{array}{r}25.132 \\
25.126\end{array}$ & $\frac{12.27}{12.27}$ & $\frac{12.27}{12.27}$ & $\frac{12.27}{1.27}$ & $\frac{12.27}{12.27}$ & $\frac{12.28}{11.27}$ & $\frac{12.28}{12.27}$ & $y$ & 6.0877 & pass \\
\hline$\angle 150$ & 25.126 & 12.27 & 12.27 & 12.27 & 12.27 & 12.27 & 12.27 & $y$ & 6.1128 & pass \\
\hline
\end{tabular}

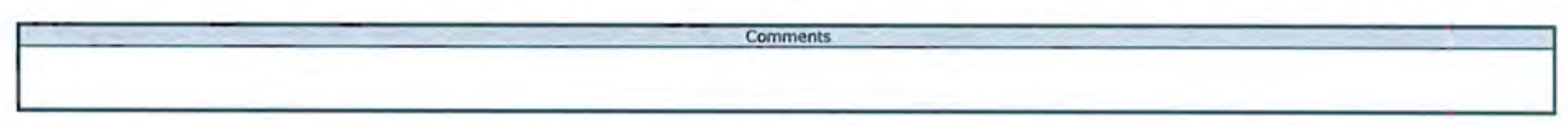

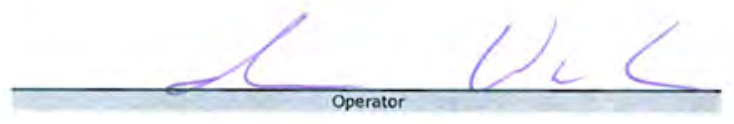
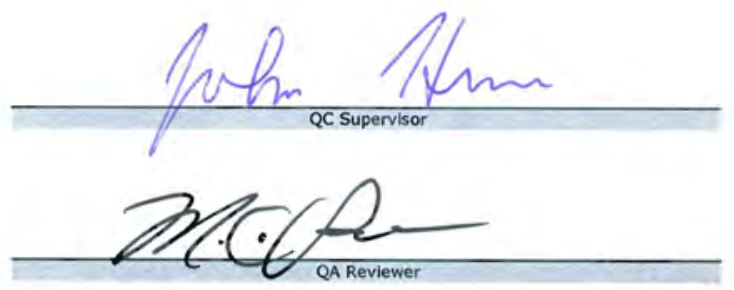

$9-14-09$

$1-12-10$

Date

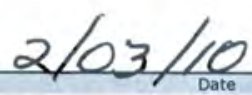




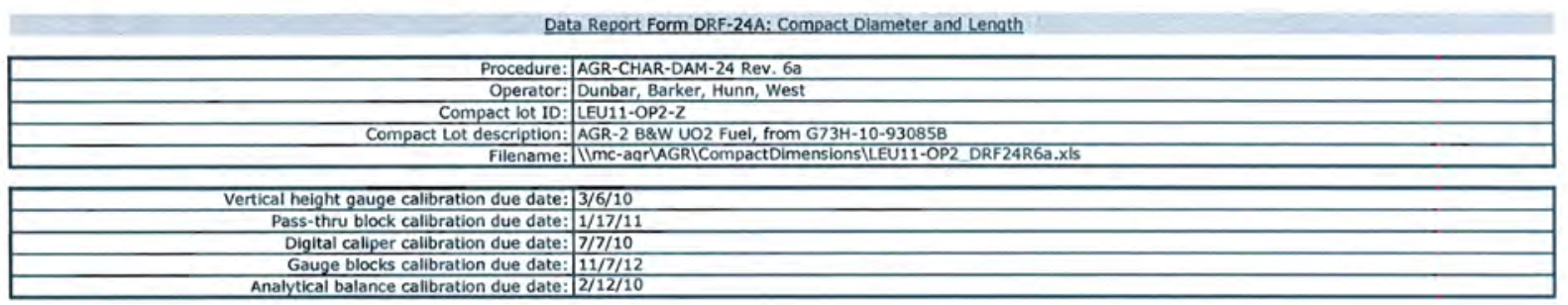

\begin{tabular}{|c|l|}
\hline Acceptance criteria for compact length: & $\geq 25.02$ and $\leq 25.40 \mathrm{~mm}$ \\
\hline Acceptance criteria for compact dlameter: & $\geq 12.22$ and $512.46 \mathrm{~mm}$ (and pass through $12.46 \mathrm{~mm}$ ring gauge) \\
\hline Acceptance criteria for compact mass: & For information only \\
\hline
\end{tabular}

\begin{tabular}{|c|c|c|c|c|c|c|c|c|c|c|}
\hline Compact & Length & \multicolumn{6}{|c|}{ Diameter $(\mathrm{mm})$} & Pass Thru? & Compact weight & Accept? \\
\hline ID Number & $\frac{(\mathrm{mm})}{25.104}$ & Top 1 & Top 2 & $\frac{\text { Middle } 1}{12,25}$ & $\frac{\text { Middle 2 }}{12.25}$ & Bottom 1 & Bottom 2 & & & \\
\hline$\frac{2151}{7152}$ & $\frac{25.104}{25.107}$ & $\frac{12.25}{12.27}$ & $\frac{12.25}{12.27}$ & $\frac{12.25}{12.28}$ & $\frac{12.25}{12.28}$ & 12.26 & $\frac{12.25}{12.28}$ & $\frac{y}{y}$ & 6.0982 & pass \\
\hline 2153 & 25.152 & 12.27 & 12.27 & 12.28 & 12.28 & $\frac{12.200}{12.27}$ & $\frac{12.28}{12.27}$ & $\frac{y}{y}$ & $\frac{6.0894}{6.1035}$ & pass \\
\hline Z154 & 25.156 & 12.28 & 12.28 & 12.28 & 12.28 & 12.27 & 12.28 & $\frac{1}{y}$ & $\frac{6.1035}{6.1087}$ & $\frac{\text { pass }}{\text { pass }}$ \\
\hline 2155 & 25.143 & 12.28 & 12.27 & 12.28 & 12.28 & 12.27 & 12.28 & $y$ & 6.0840 & pass \\
\hline Z156 & 25.125 & 12.27 & 12.27 & 12.27 & 12.27 & 12.27 & 12.27 & $y$ & 6.0941 & pass \\
\hline $\mathrm{Z157}$ & 25.130 & 12.28 & 12.28 & 12.28 & 12.27 & 12.28 & 12.29 & $y$ & 6.0844 & pass \\
\hline Z158 & 25.101 & 12.26 & 12.27 & 12.27 & 12.27 & 12.28 & 12.28 & $y$ & 6.0799 & pass \\
\hline Z159 & 25.159 & 12.29 & 12.29 & 12.28 & 12.28 & 12.28 & 12.28 & $y$ & 6.1297 & pass \\
\hline $\mathrm{Z} 160$ & 25.130 & 12.26 & 12.26 & 12.26 & 12.26 & 12.26 & 12.26 & $y$ & 6.0878 & pass \\
\hline Z161 & 25.126 & 12.29 & 12.28 & 12.28 & 12.28 & 12.28 & 12.28 & $y$ & 6.1107 & pass \\
\hline $\mathrm{Z} 162$ & 25.125 & 12.27 & 12.27 & 12.28 & 12.27 & 12.28 & 12.28 & $y$ & 6.1015 & pass \\
\hline Z163 & 25.130 & 12.27 & 12.27 & 12.27 & 12.28 & 12.27 & 12.29 & $y$ & 6.0932 & pass \\
\hline 2164 & 25.123 & 12.28 & 12.28 & 12.28 & 12.28 & 12.28 & 12.28 & $y$ & 6.0992 & pass \\
\hline 2165 & 25.083 & 12.26 & 12.26 & 12.27 & 12.28 & 12.26 & 12.26 & $y$ & 6.0912 & pass \\
\hline 2166 & 25.124 & 12.28 & 12.28 & 12.28 & 12.28 & 12.28 & 12.28 & $y$ & 6.1253 & pass \\
\hline 2167 & 25.132 & 12.25 & 12.25 & 12.26 & 12.26 & 12.25 & 12.25 & y & 6.0908 & pass \\
\hline $\mathrm{Z} 168$ & 25.124 & 12.25 & 12.25 & 12.26 & 12.26 & 12.26 & 12.26 & $y$ & 6.1011 & pass \\
\hline Z169 & 25.140 & 12.27 & 12.27 & 12.27 & 12.27 & 12.27 & 12.27 & y & 6.0785 & pass \\
\hline $\mathrm{Z} 170$ & 25.168 & 12.27 & 12.27 & 12.28 & 12.28 & 12.27 & 12.28 & $y$ & 6.1455 & pass \\
\hline $\mathrm{Z171}$ & 25.158 & 12.27 & 12.27 & 12.28 & 12.28 & 12.27 & 12.28 & $y$ & 6.1104 & pass \\
\hline$\frac{1172}{7173}$ & 25.111 & 12.27 & 12.27 & 12.27 & 12.27 & 12.27 & 12.27 & y & 6.08977 & pass \\
\hline 2173 & 23.112 & 12.21 & 12.27 & 12.28 & 12.28 & 12.28 & 12.28 & y & 6.0898 & pass \\
\hline$\frac{1174}{7175}$ & 25.144 & 12.21 & 12.27 & 12.27 & 12.26 & 12.27 & 12.27 & y & 6.0965 & pass \\
\hline 2175 & 25.143 & 12.26 & 12.26 & $\begin{array}{l}12.26 \\
2.2\end{array}$ & 12.266 & 12.25 & $\frac{12.25}{1.25}$ & $y$ & 6.1379 & pass \\
\hline$\frac{1176}{7177}$ & $\frac{25.115}{25.157}$ & 12.27 & 12.27 & 12.27 & $\frac{12.27}{127}$ & 12.26 & 12.26 & y & 6.0979 & pass \\
\hline$\frac{2177}{7178}$ & $\frac{25.107}{3}$ & 12.27 & 12.27 & 12.27 & $\frac{12.27}{127}$ & $\frac{12.27}{127}$ & $\frac{12.26}{1.28}$ & y & 6.1120 & pass \\
\hline 8178 & $\frac{23.139}{5.19}$ & 12.27 & $\frac{12.26}{12.20}$ & $\frac{12.27}{127}$ & 12.21 & $\frac{12.27}{127}$ & 12.20 & $\begin{array}{l}y \\
\end{array}$ & 6.1436 & pass \\
\hline 7813 & 23.135 & 12.27 & 12.21 & 12.27 & 12.27 & 12.27 & 12.26 & y & 0.1361 & pass \\
\hline 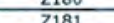 & $\frac{25.35}{55.15}$ & 12.21 & 12.21 & 12.47 & $\frac{12.21}{127}$ & 12.26 & 12.20 & y & 6.1018 & pass \\
\hline$\frac{1401}{7183}$ & $\frac{25.45}{25.12}$ & 12,270 & 12.27 & 12.200 & $\frac{1.212}{20}$ & 12.60 & 12.40 & $\frac{y}{x}$ & 0.1057 & pass \\
\hline$\frac{2102}{2183}$ & 25.150 & 12,27 & 1228 & 1228 & 128 & 12.27 & 12.278 & $\frac{y}{y}$ & 0.0971 & pass \\
\hline $\mathrm{Z} 184$ & 25 & 1228 & 1228 & 12.270 & 1220 & 1228 & 1228 & $\frac{y}{y}$ & 6.0 & pass \\
\hline 2185 & 25.105 & 12.27 & 12.28 & 12.28 & 12.28 & 12.27 & 12.27 & $\frac{1}{y}$ & 6.0766 & pass \\
\hline Z186 & 2 & 12.28 & 12.28 & 12.28 & 12.28 & 12.28 & 12.27 & $y$ & 6.0890 & pass \\
\hline 2187 & 25.159 & 12.27 & 12.27 & 12.27 & 12.27 & 12.28 & 12.28 & $y$ & 6.1087 & pass \\
\hline $\mathrm{Z188}$ & 25.133 & 12.27 & 12.27 & 12.26 & 12.27 & 12.26 & 12.27 & $y$ & 6.1028 & pass \\
\hline Z189 & 25.124 & 12.27 & 12.27 & 12.27 & 12.27 & 12.27 & 12.28 & $y$ & 6.0976 & pass \\
\hline Z190 & 25.102 & 12.27 & 12.27 & 12.28 & 12.28 & 12.28 & 12.28 & $y$ & 6.0808 & pass \\
\hline $\mathrm{Z} 191$ & 25.134 & 12.27 & 12.27 & 12.28 & 12.27 & 12.27 & 12.27 & $y$ & 6.0926 & pass \\
\hline 2192 & 25.143 & 12.26 & 12.27 & 12.27 & 12.27 & 12.26 & 12.27 & $y$ & 6.0909 & pass \\
\hline Z193 & 25.115 & 12.27 & 12.27 & 12.27 & 12.27 & 12.27 & 12.27 & $y$ & 6.0909 & pass \\
\hline Z194 & 25.107 & 12.28 & 12.27 & 12.28 & 12.28 & 12.27 & 12.27 & $y$ & 6.0972 & pass \\
\hline $\mathrm{Z} 195$ & 25.107 & 12.28 & 12.27 & 12.28 & 12.28 & 12.27 & 12.27 & $y$ & 6.0915 & pass \\
\hline Z196 & 25.132 & 12.28 & 12.28 & 12.28 & 12.28 & 12.28 & 12.27 & $y$ & 6.0895 & pass \\
\hline Z197 & 25.142 & 12.27 & 12.27 & 12.28 & 12.28 & 12.28 & 12.28 & $y$ & 6.0985 & pass \\
\hline 2998 & 25,140 & 12.28 & 12.27 & 12.28 & 12.28 & 12.28 & 12.28 & $y$ & 6.0855 & pass \\
\hline 2199 & 25.143 & 12.26 & 12.26 & 12.26 & $\frac{12.26}{126}$ & 12.27 & 12.27 & $y$ & 6.1384 & pass \\
\hline 2200 & 25.101 & 12.27 & 12.27 & 12.27 & 12.27 & 12,27 & 12.28 & $y$ & 6.0657 & pass \\
\hline
\end{tabular}

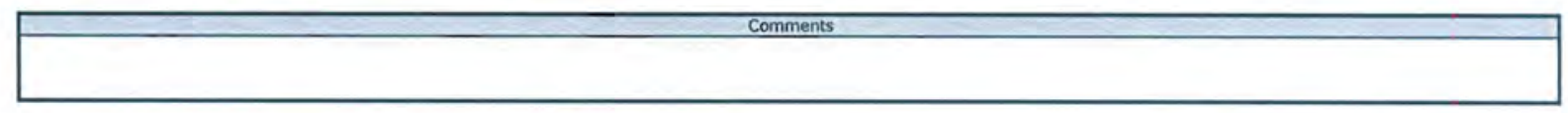

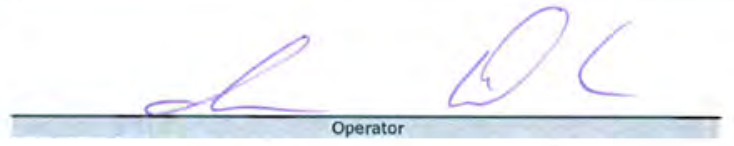
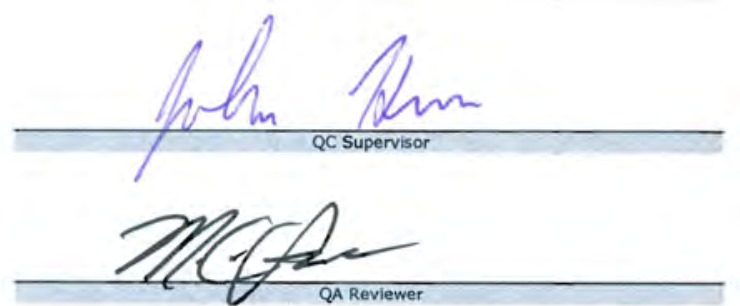

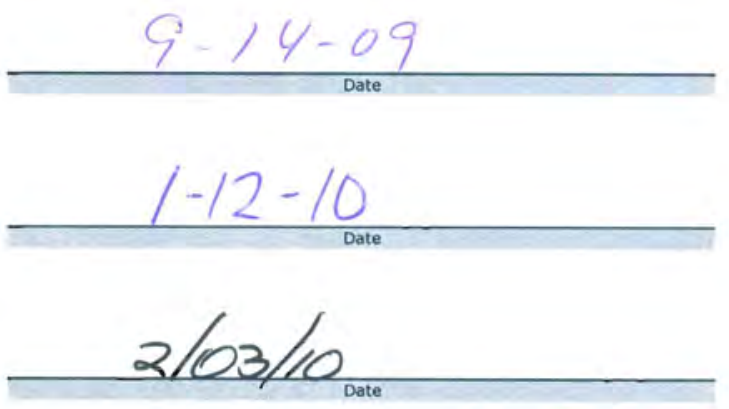


Data Report Form DRF-24A: Compact Diameter and Length

\section{Procedure: |AGR-CHAR-DAM-24 Rev. 6a}

Compact lot ID: LEU11-OP2-Z

Compact Lot description: AGR-2 B\&W UO2 Fuel, from G73H-10-93085

Filename: IIImc-aar/AGRICompactDimensionsLLEU11-OP2 DRF24R6a.xIS

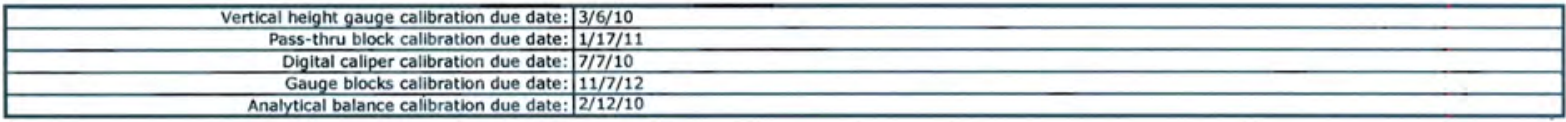

\begin{tabular}{|r|l|}
\hline Acceptance criteria for compact length: & $\geq 25.02$ and $\leq 25.40 \mathrm{~mm}$ \\
\hline Acceptance criteria for compact diameters & $\geq 12.22$ and $\leq 12.46 \mathrm{~mm}$ (and pass through $12.46 \mathrm{~mm}$ ring gauge) \\
\hline Acceptance criteria for compact mass: & For information only \\
\hline
\end{tabular}

\begin{tabular}{|c|c|c|c|c|c|c|c|c|c|c|}
\hline $\begin{array}{l}\text { Compact } \\
\text { ID Numbe }\end{array}$ & $\begin{array}{c}\text { Length } \\
(\mathrm{mm})\end{array}$ & & & $\begin{array}{l}\text { Diam } \\
\text { Mothe }\end{array}$ & $\mathrm{er}(\mathrm{mm})$ & & & Pass Thru? & Compact weight & Accept? \\
\hline 2201 & 25.151 & Top 1 & Top 22 & Middle 1 & $\frac{\text { Middle } 2}{12.28}$ & $\frac{\text { Bottom } 1}{12.28}$ & Bottom 2 & $\frac{(\gamma \text { or } N)}{y}$ & & $\frac{\text { (pass or fail) }}{\text { pass }}$ \\
\hline 2202 & 25.142 & 12.25 & 12.25 & 12.25 & 12.25 & 12.25 & 12.25 & $\frac{y}{y}$ & 6.1375 & pass \\
\hline 2203 & 25.120 & 12.27 & 12.27 & 12.27 & 12.28 & 12.27 & 12.28 & & 6.0864 & pass \\
\hline 2204 & & & & & & & & & & \\
\hline 2205 & & & & & & & & & & \\
\hline 2206 & & & & & & & & & & \\
\hline Z207 & & & & & & & & & & \\
\hline 2208 & & & & & & & & & & \\
\hline $\begin{array}{l}Z 209 \\
\mathbf{Z 2 1 0}\end{array}$ & & & & & & & & & & \\
\hline$\frac{Z 210}{Z 211}$ & & & & & & & & & & \\
\hline$\frac{2211}{Z 212}$ & & & & & & & & - & & \\
\hline 2213 & & & & & & & & & & \\
\hline 2214 & & & & & & & & & & \\
\hline 2215 & & & & & & & & & & \\
\hline & & & & & & & & & & \\
\hline & & & & & & & & & & \\
\hline 2219 & & & & & & & & & & \\
\hline 2220 & & & & & & & & & & \\
\hline 2221 & & & & & & & & & & \\
\hline$\frac{2222}{7223}$ & & & & & & & & & & \\
\hline 2223 & & & & & & & & & & \\
\hline$\frac{2224}{2225}$ & & & & & & & & & & \\
\hline 2226 & & & & & & & & & & \\
\hline 2227 & & & & & & & & & & \\
\hline 2228 & & & & & & & & & & \\
\hline 2229 & & & & & & & & & & \\
\hline 2230 & & & & & & & & & & \\
\hline 2231 & & & & & & & & & & \\
\hline 2232 & & & & & & & & & & \\
\hline 2233 & & & & & & & & & & \\
\hline 2234 & & & & & & & & & & \\
\hline 2235 & & & & & & & & & & \\
\hline 2236 & & & & & & & & & & \\
\hline 2237 & & & & & & & & & & \\
\hline 2238 & & & & & & & & & & \\
\hline 2239 & & & & & & & & & & \\
\hline 2241 & & & & & & & & & & \\
\hline Z242 & & & & & & & & & & \\
\hline 2243 & & & & & & & & & & \\
\hline Z2244 & & & & & & & & & & \\
\hline 2245 & & & & & & & & & & \\
\hline & & & & & & & & & & \\
\hline 2247 & & & & & & & & & & \\
\hline$\frac{2248}{Z 249}$ & & & & & & & & & & \\
\hline 2250 & & & & & & & & & & \\
\hline
\end{tabular}

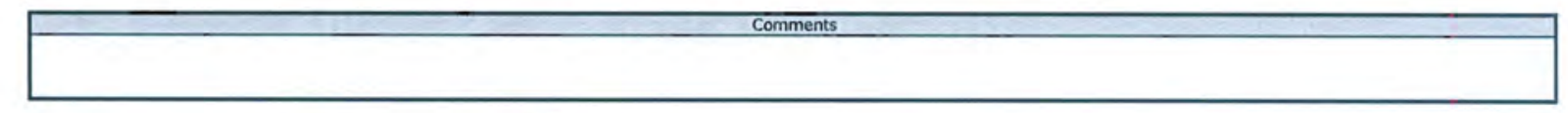

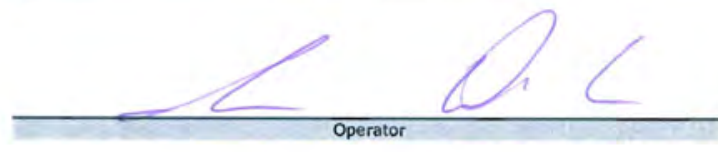

$10 \mathrm{hm}$ ecsupervisor

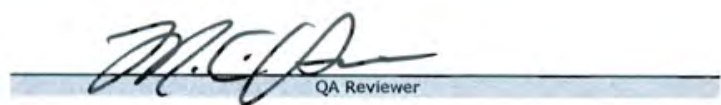

$$
9-14-09
$$

$$
1-12-10
$$

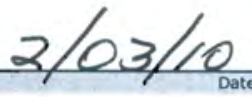


Data Report Form DRF-24B: Compact Matrix Density

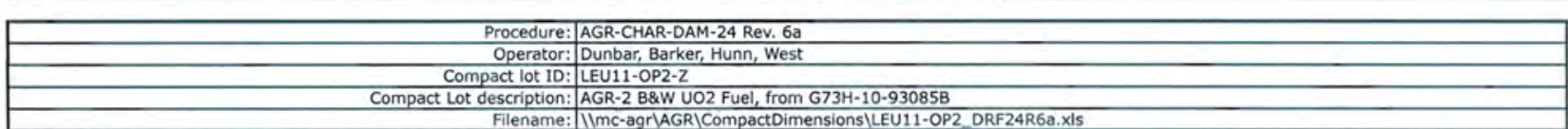

\begin{tabular}{|r|l|}
\hline Average weight per TRISO particle $(g):$ & $1.462 \mathrm{E}-03$ \\
\hline Average weight per overcoated particle $(\mathrm{g}):$ & $4.053 \mathrm{E}-03$ \\
\hline Average TRISO particle volume $(\mathrm{cm} 3):$ & $4.450 \mathrm{E}-04$ \\
\hline
\end{tabular}

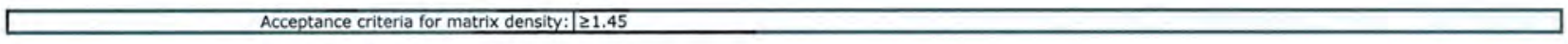

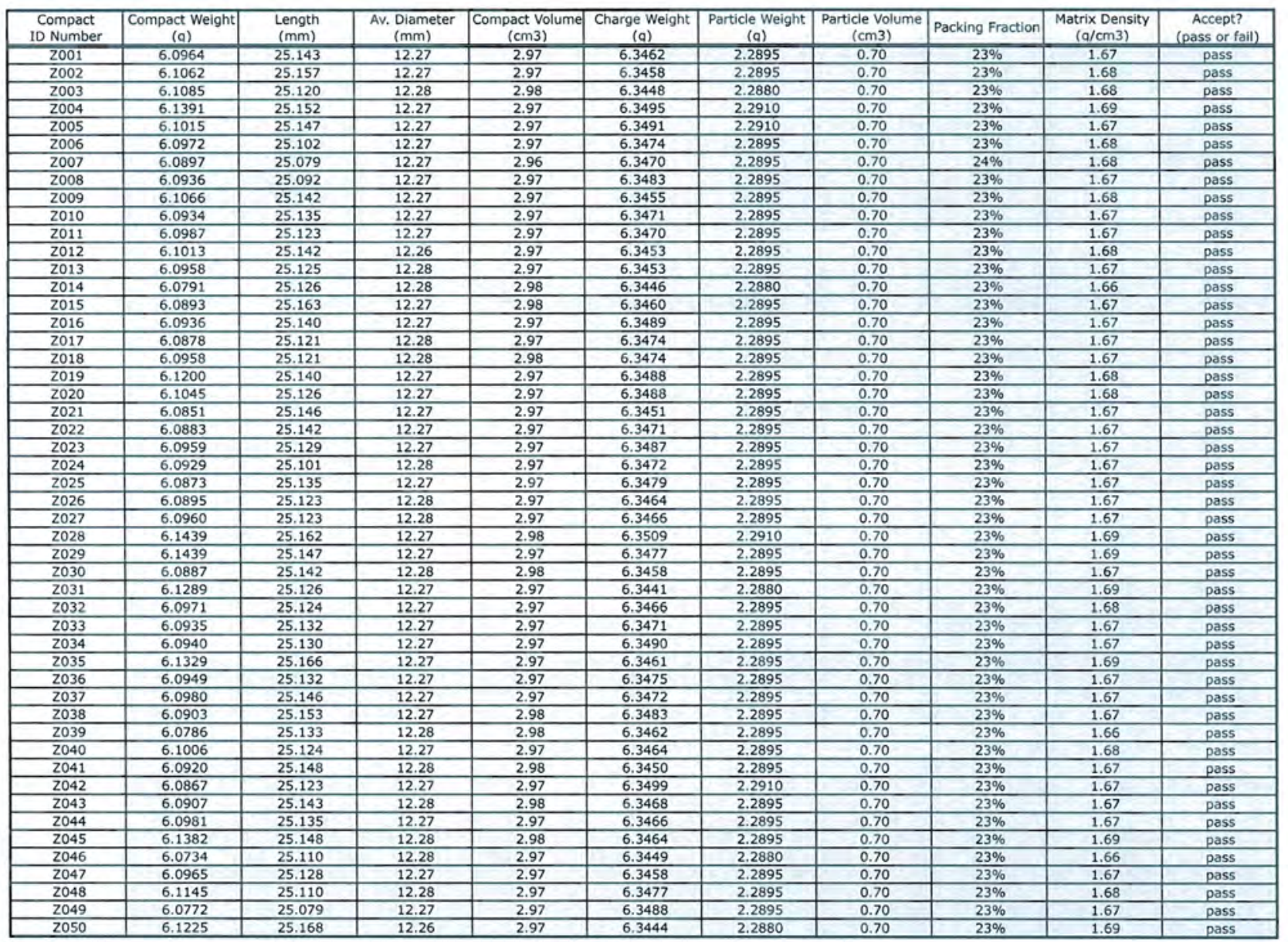

Comments

Average weight per overcoated particle from combined results of 2 independent measurements (W09081401 and W09081402).

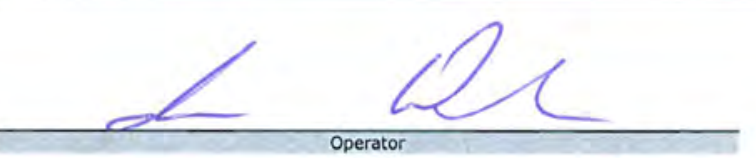

4 oc supervisor

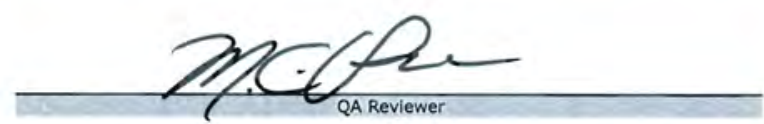

$9-14-09$

$1-12-10$

$2 / 03 / 10$ 


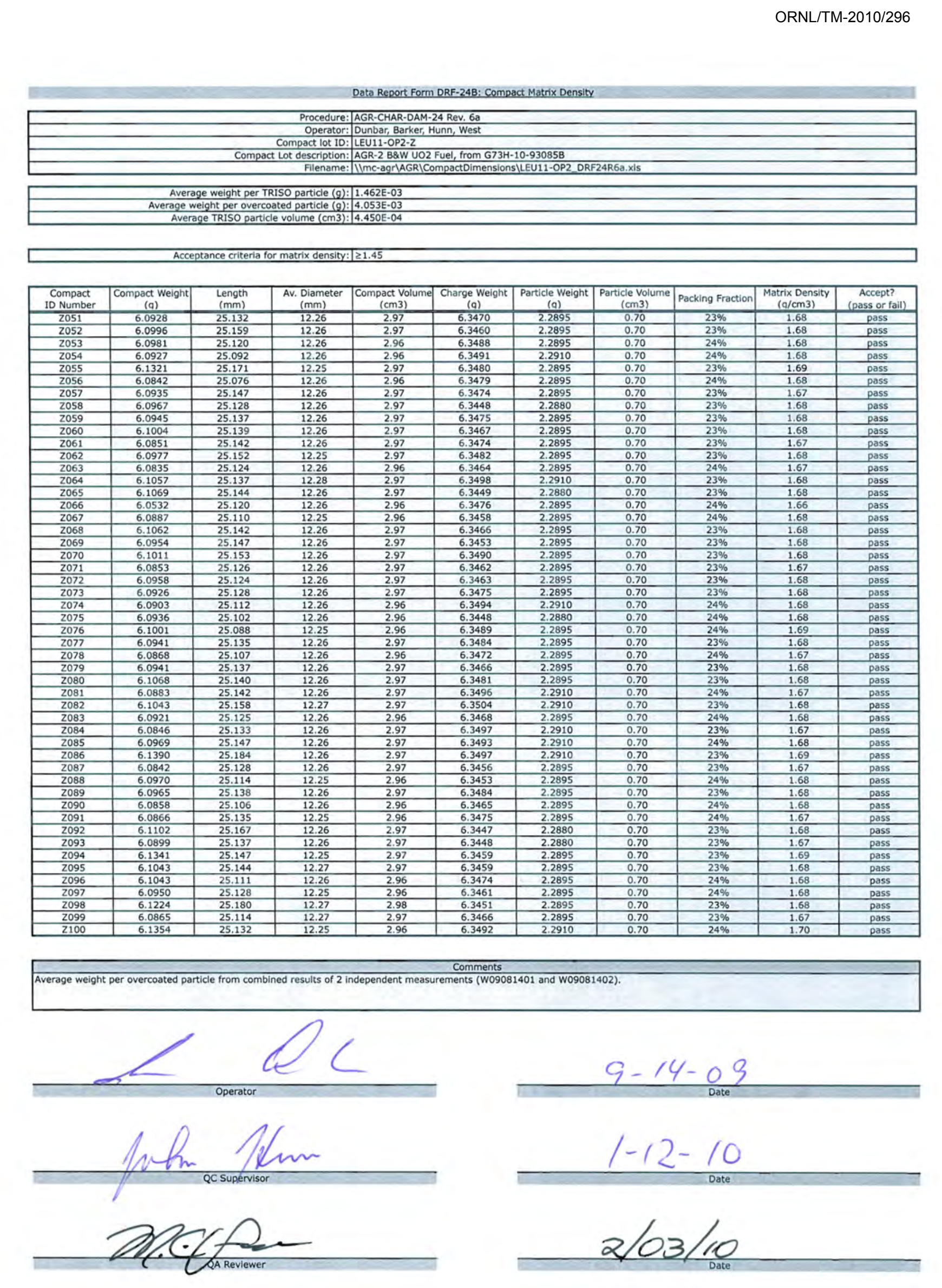


ORNL/TM-2010/296

Data Report Form DRF-24B: Compact Matrix Density

Procedure: AGR-CHAR-DAM-24 Rev. 6 a

Operator: Dunbar, Barker, Hin, West Compact lot ID: LEU11-OP2-Z

Compact Lot description: $A G R-2$ B\&W UO2 Fuel, from G73H-10-93085B

Filename: \IImc-agr\AGR\CompactDimensions\LEU11-OP2_DRF24R6a,xls

Average weight per TRISO particle (g): $1.462 \mathrm{E}-03$

\begin{tabular}{lll} 
Average TRISO particle volume $(\mathrm{cm} 3):$ & $4.450 \mathrm{E}-04$ \\
\hline
\end{tabular}

Acceptance criteria for matrix density: $\geq 1.45$

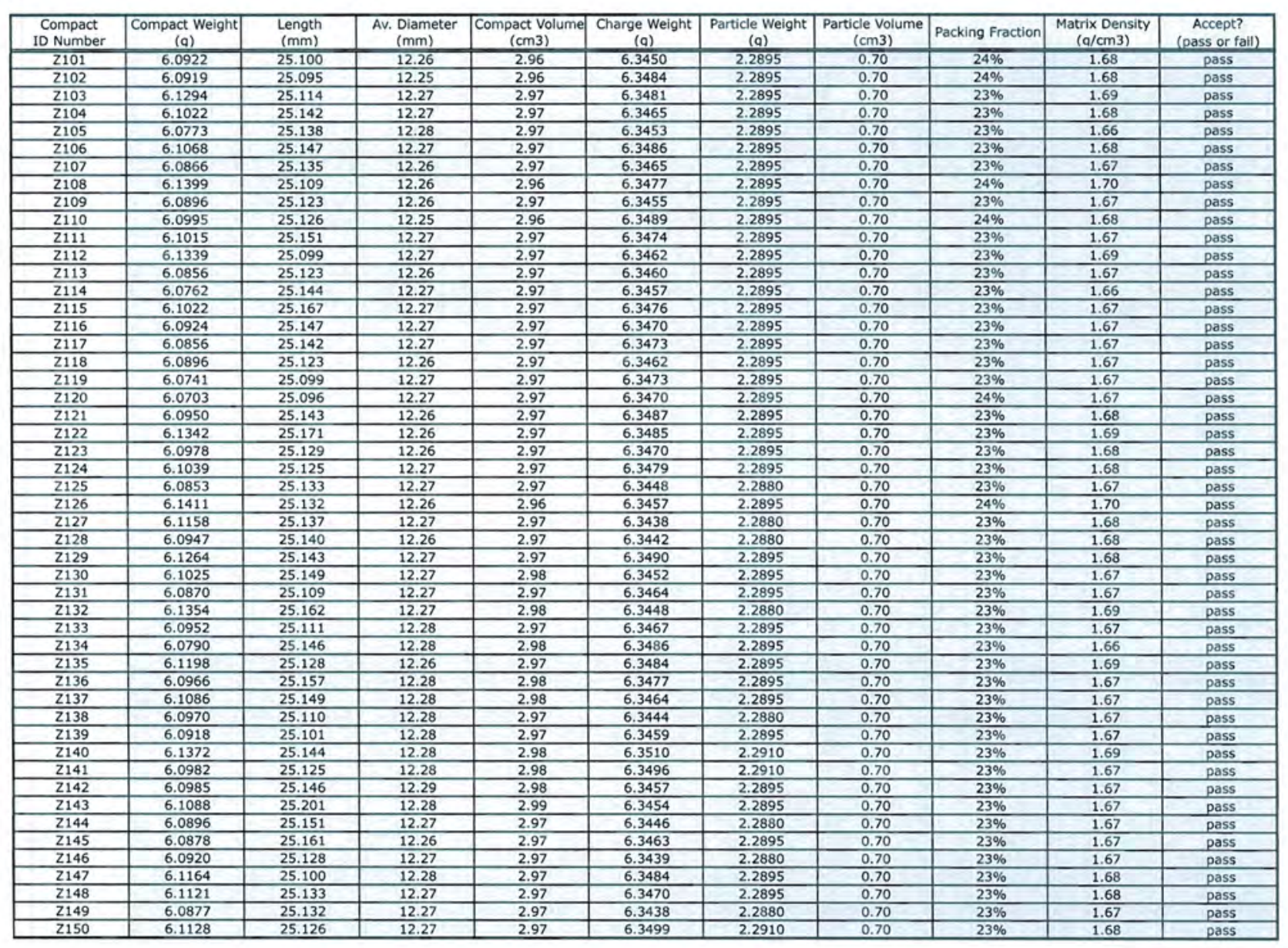

\begin{tabular}{|c|c|c|}
\hline & Comments \\
\hline Average weight per overcoated particle from combined results of 2 independent measurements (W09081401 and W09081402).
\end{tabular}

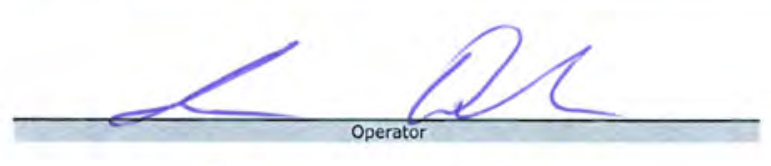

$9-14-09$
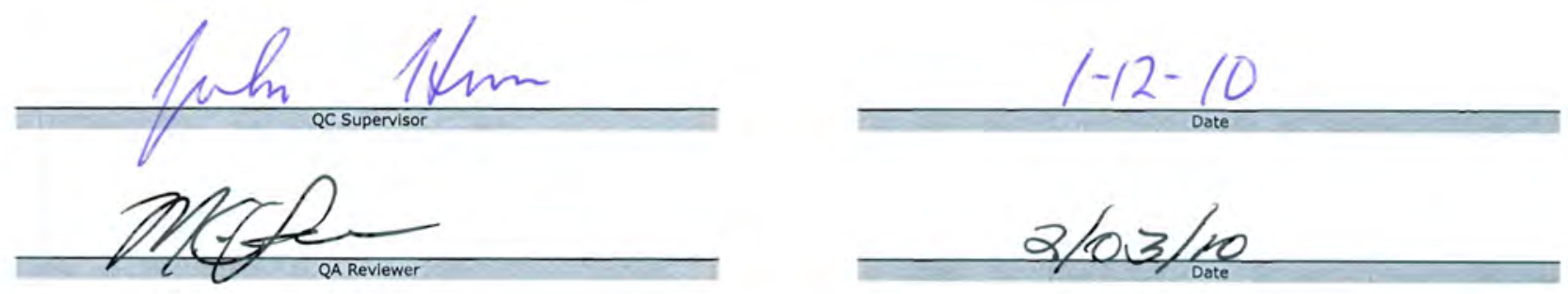

52 
Data Report Form DRF-24B: Compact Matrix Density

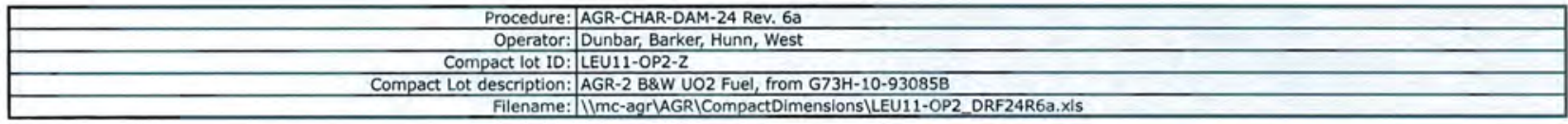

\begin{tabular}{|c|}
\hline Average weight per TRISO particle $(\mathrm{g}): 1.462 \mathrm{E}-03$ \\
\hline Average weight per overcoated particle $(\mathrm{g}): 4.053 \mathrm{E}-03$ \\
\hline Average TRISO particle volume $(\mathrm{cm} 3): 4.450 \mathrm{E}-04$ \\
\hline
\end{tabular}

\section{Acceptance criteria for matrix density: $\mid \geq 1.45$}

\begin{tabular}{|c|c|c|c|c|c|c|c|c|c|c|}
\hline $\begin{array}{l}\text { Compact } \\
\text { ID Number }\end{array}$ & \begin{tabular}{|l|} 
Compact Weight \\
(a)
\end{tabular} & $\begin{array}{l}\text { Length } \\
(\mathrm{mm})\end{array}$ & $\begin{array}{l}\text { Av. Diameter } \\
(\mathrm{mm})\end{array}$ & \begin{tabular}{|c|} 
Compact Volume \\
$(\mathrm{cm} 3)$
\end{tabular} & $\begin{array}{l}\text { Charge Weight } \\
\text { (a) }\end{array}$ & $\begin{array}{l}\text { Particle Weight } \\
\text { (a) }\end{array}$ & $\begin{array}{l}\text { Particle Volume } \\
\text { (cm3) }\end{array}$ & Packing Fraction & $\begin{array}{l}\text { Matrix Density } \\
(0 / \mathrm{cm} 3)\end{array}$ & $\begin{array}{c}\text { Accept? } \\
\text { (pass or fail) }\end{array}$ \\
\hline 2151 & 6.0982 & 25.104 & 12.25 & 2.96 & 6.3449 & 2.2880 & 0.70 & $24 \%$ & 1.68 & \\
\hline 2152 & 6.0894 & 25.107 & 12.28 & 2.97 & 6.3455 & 2.2895 & 0.70 & $23 \%$ & 1.67 & pass \\
\hline Z153 & 6.1035 & 25.152 & 12.27 & 2.98 & 6.3478 & 2.2895 & 0.70 & $23 \%$ & 1.67 & pass \\
\hline $\mathrm{Z154}$ & 6.1087 & 25.156 & 12.28 & 2.98 & 6.3467 & 2.2895 & 0.70 & $23 \%$ & 1.67 & pass \\
\hline 2155 & 6.0840 & 25.143 & 12.28 & 2.98 & 6.3450 & 2.2895 & 0.70 & $23 \%$ & 1.66 & pass \\
\hline 2156 & 6.0941 & 25.125 & 12.27 & 2.97 & 6.3487 & 2.2895 & 0.70 & $23 \%$ & 1.67 & pass \\
\hline $\mathrm{Z157}$ & 6.0844 & 25.130 & 12.28 & 2.98 & 6.3491 & 2.2910 & 0.70 & $23 \%$ & 1.66 & pass \\
\hline 2158 & 6.0799 & 25.101 & 12.27 & 2.97 & 6.3452 & 2.2895 & 0.70 & $23 \%$ & 1.67 & pass \\
\hline 2159 & 6.1297 & 25.159 & 12.28 & 2.98 & 6.3460 & 2.2895 & 0.70 & $23 \%$ & 1.68 & pass \\
\hline 2160 & 6.0878 & 25.130 & 12.26 & 2.97 & 6.3460 & 2.2895 & 0.70 & $23 \%$ & 1.67 & pass \\
\hline 2161 & 6.1107 & 25.126 & 12.28 & 2.98 & 6.3490 & 2.2895 & 0.70 & $23 \%$ & 1.68 & pass \\
\hline $\mathrm{Z162}$ & 6.1015 & 25.125 & 12.28 & 2.97 & 6.3470 & 2.2895 & 0.70 & $23 \%$ & 1.67 & pass \\
\hline 2163 & 6.0932 & 25.130 & 12.28 & 2.97 & 6.3462 & 2.2895 & 0.70 & $23 \%$ & 1.67 & pass \\
\hline 2164 & 6.0992 & 25.123 & 12.28 & 2.98 & 6.3461 & 2.2895 & 0.70 & $23 \%$ & 1.67 & pass \\
\hline 2165 & 6.0912 & 25.083 & 12.27 & 2.96 & 6.3469 & 2.2895 & 0.70 & $24 \%$ & 1.68 & pass \\
\hline 2166 & 6.1253 & 25.124 & 12.28 & 2.98 & 6.3447 & 2.2880 & 0.70 & $23 \%$ & 1.68 & pass \\
\hline Z167 & 6.0908 & 25.132 & 12.25 & 2.96 & 6.3487 & 2.2895 & 0.70 & $24 \%$ & 1.68 & pass \\
\hline $\mathrm{Z168}$ & 6.1011 & 25.124 & 12.26 & 2.96 & 6.3489 & 2.2895 & 0.70 & $24 \%$ & 1.68 & pass \\
\hline Z169 & 6.0785 & 25.140 & 12.27 & 2.97 & 6.3463 & 2.2895 & 0.70 & $23 \%$ & 1.66 & pass \\
\hline 2170 & 6.1455 & 25.168 & 12.28 & 2.98 & 6.3486 & 2.2895 & 0.70 & $23 \%$ & 1.69 & pass \\
\hline $\mathrm{Z171}$ & 6.1104 & 25.158 & 12.28 & 2.98 & 6.3490 & 2.2895 & 0.70 & $23 \%$ & 1.68 & pass \\
\hline $\mathrm{Z172}$ & 6.0897 & 25.111 & 12.27 & 2.97 & 6.3458 & 2.2895 & 0.70 & $23 \%$ & 1.67 & pass \\
\hline 2173 & 6.0898 & 25.112 & 12.28 & 2.97 & 6.3488 & 2.2895 & 0.70 & $23 \%$ & 1.67 & pass \\
\hline Z174 & 6.0965 & 25.144 & 12.27 & 2.97 & 6.3470 & 2.2895 & 0.70 & $23 \%$ & 1.67 & pass \\
\hline 2175 & 6.1379 & 25.143 & 12.26 & 2.97 & 6.3464 & 2.2895 & 0.70 & $23 \%$ & 1.70 & pass \\
\hline 2176 & 6.0979 & 25.115 & 12.27 & 2.97 & 6.3479 & 2.2895 & 0.70 & $23 \%$ & 1.68 & pass \\
\hline 2177 & 6.1120 & 25.107 & 12.27 & 2.97 & 6.3475 & 2.2895 & 0.70 & $23 \%$ & 1.68 & pass \\
\hline 2178 & 6.1436 & 25.137 & 12.27 & $\frac{2.37}{2.97}$ & 6.3465 & 2.2895 & 0.70 & $23 \%$ & 1.69 & pass \\
\hline 2179 & 6.1361 & 25.135 & 12.27 & 2.97 & 6.3459 & 2.2895 & 0.70 & $23 \%$ & 1.69 & pass \\
\hline 2180 & 6.1018 & 25.153 & 12.27 & 2.98 & 6.3487 & 2.2895 & 0.70 & $23 \%$ & 1.67 & pass \\
\hline 2181 & 6.1057 & 25.146 & 12.27 & 2.97 & 6.3466 & 2.2895 & 0.70 & $23 \%$ & 1.68 & pass \\
\hline $\mathrm{Z182}$ & 6.0987 & 25.120 & 12.27 & 2.97 & 6.3499 & 2.2910 & 0.70 & $23 \%$ & 1.68 & pass \\
\hline 2183 & 6.0887 & 25.152 & 12.28 & 2.98 & 6.3483 & 2.2895 & 0.70 & $23 \%$ & 1.67 & pass \\
\hline Z184 & 6.0845 & 25.159 & 12.28 & 2.98 & 6.3462 & 2.2895 & 0.70 & $23 \%$ & 1.66 & pass \\
\hline Z185 & 6.0766 & 25.105 & 12.28 & 2.97 & 6.3471 & 2.2895 & 0.70 & $23 \%$ & 1.67 & pass \\
\hline $\mathrm{Z186}$ & 6.0890 & 25.110 & 12.28 & 2.97 & 6.3474 & 2.2895 & 0.70 & $23 \%$ & 1.67 & pass \\
\hline $\mathrm{Z187}$ & 6.1087 & 25.159 & 12.27 & 2.98 & 6.3448 & 2.2880 & 0.70 & $23 \%$ & 1.68 & pass \\
\hline 2188 & $\frac{6.1001}{6.1028}$ & $\frac{25.159}{25.133}$ & $\frac{1.27}{12.27}$ & $\frac{2.960}{2.97}$ & $\frac{6.3448}{6.3470}$ & $\frac{2.2800}{2.2895}$ & 0.70 & $23 \%$ & 1.68 & $\frac{\text { pass }}{\text { pass }}$ \\
\hline Z189 & 6.0976 & 25.124 & 12.27 & 2.97 & 6.3476 & 2.2895 & 0.70 & $23 \%$ & 1.67 & pass \\
\hline $\mathrm{Z190}$ & 6.0808 & 25.102 & 12.28 & 2.97 & 6.3477 & 2.2895 & 0.70 & $23 \%$ & 1.67 & pass \\
\hline $\mathrm{Z} 191$ & 6.0926 & 25.134 & 12.27 & 2.97 & 6.3468 & 2.2895 & 0.70 & $23 \%$ & 1.67 & pass \\
\hline Z192 & 6.0909 & 25.143 & 12.27 & 2.97 & 6.3463 & 2.2895 & 0.70 & $23 \%$ & 1.67 & pass \\
\hline $\mathrm{Z} 193$ & 6.0909 & 25.115 & 12.27 & 2.97 & 6.3445 & 2.2880 & 0.70 & $23 \%$ & 1.67 & pass \\
\hline Z194 & 6.0972 & 25.107 & 12.28 & 2.97 & 6.3485 & 2.2895 & 0.70 & $23 \%$ & $\begin{array}{l}1.07 \\
1.67\end{array}$ & $\begin{array}{l}\text { pass } \\
\text { pass }\end{array}$ \\
\hline 2195 & 6.0915 & 25.107 & 12.28 & 2.97 & 6.3484 & 2.2895 & 0.70 & $23 \%$ & 1.67 & pass \\
\hline Z196 & 6.0895 & 25.1 & 12.28 & 2.98 & 6.3465 & 2.28 & 0.70 & $23 \%$ & 1.07 & pass \\
\hline Z197 & 6.0985 & & 12 & 2.98 & 6.36 & 2.28 & 0. & $23 \%$ & 1.67 & pass \\
\hline $\mathrm{Z} 198$ & 6.0855 & & & 2.98 & 6.36 & 2.2895 & 0.70 & $23 \%$ & 1.67 & pass \\
\hline Z199 & 6.1384 & 25.143 & 12.26 & 2.97 & 6.34700 & 2.2895 & 0.70 & $23 \%$ & 1.69 & pass \\
\hline 2200 & 6.0657 & 25.101 & 12.27 & 2.97 & 6.34779 & $\frac{2.2850}{2.2895}$ & 0.70 & $23 \%$ & 1.66 & pass \\
\hline
\end{tabular}

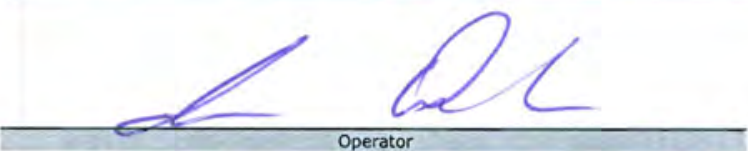

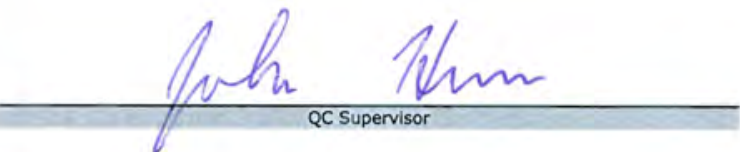

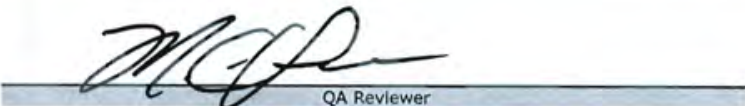

$9-14-0 ?$

$1-12-10$

Date

$2 / 03 / 10$ 
Data Report Form DRF-24B: Compact Matrix Density

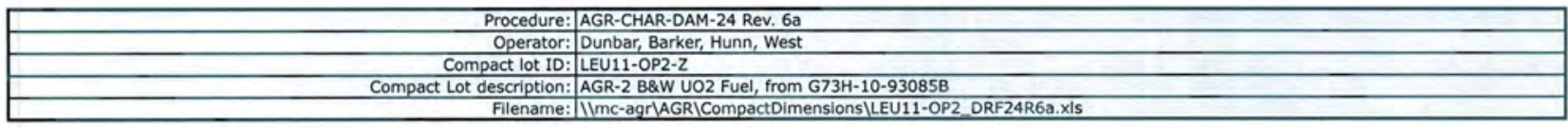

\begin{tabular}{|c|c|}
\hline Average weight per TRISO particle $(g):$ & $1.462 \mathrm{E}-03$ \\
\hline Average weight per overcoated particle $(g):$ & $4.053 \mathrm{E}-03$ \\
\hline Average TRISO particle volume $(\mathrm{cm} 3):$ & $4.450 \mathrm{E}-04$ \\
\hline
\end{tabular}

Acceptance criteria for matrix density: $2 \geq 1.45$

\begin{tabular}{|c|c|c|c|c|c|c|c|c|c|c|}
\hline $\begin{array}{l}\text { Compact } \\
\text { ID Number }\end{array}$ & $\begin{array}{l}\text { Compact Weight } \\
\text { (a) }\end{array}$ & $\begin{array}{l}\text { Length } \\
(\mathrm{mm})\end{array}$ & $\begin{array}{l}\text { Av. Diameter } \\
(\mathrm{mm})\end{array}$ & $\begin{array}{c}\text { Compact Volume } \\
(\mathrm{cm} 3)\end{array}$ & $\begin{array}{l}\text { Charge Weight } \\
\text { (a) }\end{array}$ & \begin{tabular}{l|} 
Particle Weight \\
(a)
\end{tabular} & $\begin{array}{l}\text { Particle Volume } \\
(\mathrm{cm} 3)\end{array}$ & Packing Fraction & $\begin{array}{l}\text { Matrix Density } \\
(\mathrm{a} / \mathrm{cm} 3)\end{array}$ & $\begin{array}{c}\text { Accept? } \\
\text { (pass or fail) }\end{array}$ \\
\hline 2201 & 6.1076 & 25.151 & 12.28 & 2.98 & 6.3493 & 2.2910 & 0.70 & $23 \%$ & 1.67 & pass \\
\hline 2202 & 6.1375 & 25.142 & 12.25 & 2.96 & 6.3470 & 2.2895 & 0.70 & $24 \%$ & 1.70 & pass \\
\hline 2203 & 6.0864 & 25.120 & 12.27 & 2.97 & 6.3462 & 2.2895 & 0.70 & $23 \%$ & 1.67 & pass \\
\hline 2204 & & & & & & & & & & \\
\hline 2205 & & & & & & & & & & \\
\hline 2206 & & & & & & & & & & \\
\hline$\frac{Z 207}{Z 208}$ & & & & & & & & & & \\
\hline$\frac{2208}{Z 209}$ & & & & & & & & & & \\
\hline 2210 & & & & & & & & & & \\
\hline 2211 & & & & & & & & & & \\
\hline 2212 & & & & & & & & & & \\
\hline 2213 & & & & & & & & & & \\
\hline $\mathrm{Z214}$ & & & & & & & & & & \\
\hline 2215 & & & & & & & & & & \\
\hline 2216 & & & & & & & & & & \\
\hline 2217 & & & & & & & & & & \\
\hline $\mathrm{Z218}$ & & & & & & & & & & \\
\hline 2219 & & & & & & & & & & \\
\hline 2220 & & & & & & & & & & \\
\hline 2221 & & & & & & & & & & \\
\hline 2222 & & & & & & & & & & \\
\hline 2223 & & & & & & & & & & \\
\hline 2224 & & & & & & & & & & \\
\hline 2225 & & & & & & & & & & \\
\hline 2226 & & & & & & & & & & \\
\hline 2227 & & & & & & & & & & \\
\hline 2228 & & & & & & & & & & \\
\hline 2229 & & & & & & & & & & \\
\hline 2230 & & & & & & & & & & \\
\hline 2231 & & & & & & & 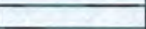 & 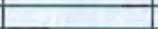 & & \\
\hline $\mathrm{Z232}$ & & & & & & & & & & 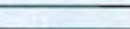 \\
\hline 2233 & & & & & & & & & & \\
\hline $\mathrm{Z234}$ & & & & & & & & 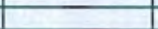 & & \\
\hline 2235 & & & & & & & & 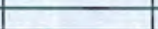 & 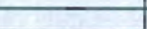 & \\
\hline $\mathrm{Z236}$ & & & & & & & & 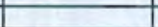 & & \\
\hline $\mathrm{Z237}$ & & & & & & & & & & \\
\hline $\mathrm{Z238}$ & & & & & & & & & & \\
\hline $\mathrm{Z239}$ & & & & & & & & & & \\
\hline 2240 & & & & & & & & & & \\
\hline 2241 & & & & & & & & & & \\
\hline 2242 & & & & & & & & 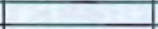 & & \\
\hline 2243 & & & 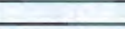 & & & & & & & \\
\hline 2244 & & 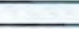 & + & & & & & 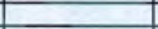 & & \\
\hline 2245 & & & & & & & & & & \\
\hline 2246 & & & & & & & & & & \\
\hline 2247 & & & & & & & & & & \\
\hline$Z 248$ & & & & & & & & & & 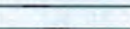 \\
\hline 2249 & & & & & & & & & 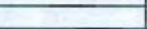 & \\
\hline 2250 & & & & & & & & & & \\
\hline
\end{tabular}

Average weight per overcoated particle from combined results of 2 independent measurements (W099081401 and W09081402).

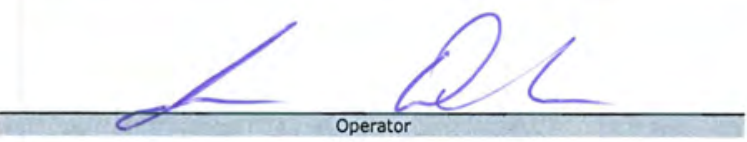

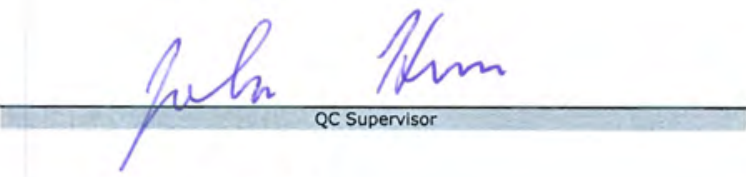

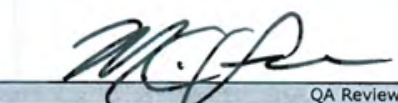

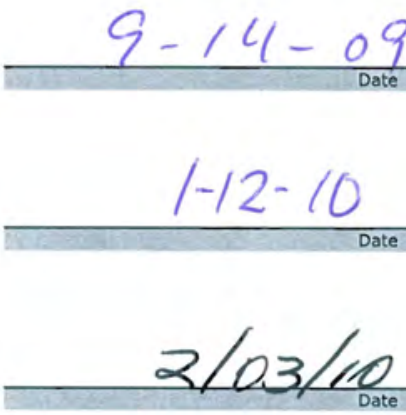


Data Report Form DRF-24C: Compact Tracking

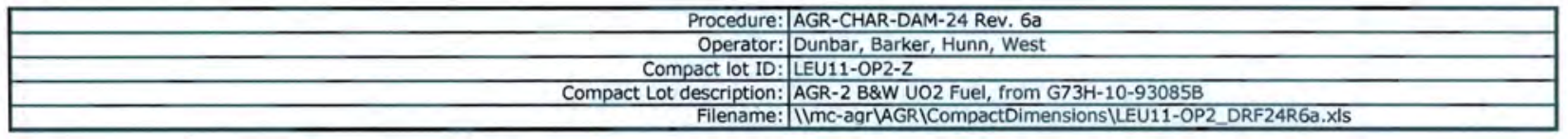

\begin{tabular}{|c|c|}
\hline $\begin{array}{l}\text { Compact } \\
\text { Z Number }\end{array}$ & $\begin{array}{c}\text { Compact G } \\
\text { Number }\end{array}$ \\
\hline$Z 001$ & $\mathrm{G} 021$ \\
\hline$Z 002$ & G106 \\
\hline Z003 & G197 \\
\hline 2004 & G087 \\
\hline 2005 & G130 \\
\hline 2006 & G060 \\
\hline Z007 & $\mathrm{G} 047$ \\
\hline 2008 & G147 \\
\hline 2009 & G101 \\
\hline 2010 & G071 \\
\hline$Z 011$ & G035 \\
\hline Z012 & G057 \\
\hline Z013 & G195 \\
\hline 2014 & G180 \\
\hline 2015 & G114 \\
\hline$Z 016$ & G078 \\
\hline$Z 017$ & G164 \\
\hline 2018 & G206 \\
\hline Z019 & G107 \\
\hline 2020 & G037 \\
\hline$Z 021$ & G172 \\
\hline 2022 & G118 \\
\hline 2023 & G132 \\
\hline$Z 024$ & G139 \\
\hline 2025 & G154 \\
\hline 2026 & G138 \\
\hline Z027 & G042 \\
\hline 2028 & G085 \\
\hline 2029 & G084 \\
\hline 2030 & G140 \\
\hline 2031 & G089 \\
\hline 2032 & G129 \\
\hline 2033 & G117 \\
\hline 2034 & G111 \\
\hline$Z 035$ & G093 \\
\hline 2036 & $\mathrm{G} 003$ \\
\hline 2037 & G009 \\
\hline Z038 & G108 \\
\hline 2039 & G160 \\
\hline $\mathrm{ZO40}$ & G128 \\
\hline Z041 & G122 \\
\hline 2042 & 6065 \\
\hline Z043 & G020 \\
\hline$Z 044$ & G133 \\
\hline 2045 & G217 \\
\hline 2046 & G157 \\
\hline 2047 & G193 \\
\hline$Z 048$ & $\mathrm{G} 201$ \\
\hline Z049 & G155 \\
\hline 2050 & G017 \\
\hline
\end{tabular}

\begin{tabular}{|c|c|}
\hline $\begin{array}{l}\text { Compact } \\
Z \text { Number }\end{array}$ & $\begin{array}{c}\text { Compact G } \\
\text { Number }\end{array}$ \\
\hline 2051 & $\mathrm{G} 036$ \\
\hline 2052 & G012 \\
\hline 2053 & G041 \\
\hline Z054 & G171 \\
\hline 2055 & G098 \\
\hline 2056 & G156 \\
\hline 2057 & G124 \\
\hline Z058 & G177 \\
\hline$Z 059$ & G008 \\
\hline 2060 & G007 \\
\hline$Z 061$ & G185 \\
\hline Z062 & G059 \\
\hline 2063 & G170 \\
\hline 2064 & G209 \\
\hline 2065 & G062 \\
\hline 2066 & G046 \\
\hline 2067 & G135 \\
\hline Z068 & G070 \\
\hline Z069 & G125 \\
\hline$Z 070$ & G202 \\
\hline 2071 & G162 \\
\hline Z072 & G194 \\
\hline 2073 & G167 \\
\hline Z074 & G153 \\
\hline 2075 & G121 \\
\hline 2076 & G025 \\
\hline$Z 077$ & G022 \\
\hline Z078 & G187 \\
\hline Z079 & G040 \\
\hline$Z 080$ & G011 \\
\hline Z081 & G045 \\
\hline$Z 082$ & G212 \\
\hline Z083 & G145 \\
\hline 2084 & G188 \\
\hline 2085 & G024 \\
\hline 2086 & G096 \\
\hline 2087 & G176 \\
\hline$Z 088$ & G056 \\
\hline Z089 & G148 \\
\hline 2090 & G069 \\
\hline$Z 091$ & G005 \\
\hline 2092 & $\mathrm{G} 032$ \\
\hline 2093 & G141 \\
\hline Z094 & G091 \\
\hline 2095 & G211 \\
\hline 2096 & G126 \\
\hline 2097 & G075 \\
\hline 2098 & G214 \\
\hline 2099 & G204 \\
\hline $\mathrm{Z} 100$ & G092 \\
\hline
\end{tabular}

\begin{tabular}{|c|c|}
\hline $\begin{array}{l}\text { Compact } \\
Z \text { Number }\end{array}$ & $\begin{array}{l}\text { Compact G } \\
\text { Number }\end{array}$ \\
\hline $\mathrm{Z101}$ & G123 \\
\hline $\mathrm{Z102}$ & G049 \\
\hline $\mathrm{Z} 103$ & G219 \\
\hline Z104 & G144 \\
\hline Z105 & G066 \\
\hline Z106 & G010 \\
\hline$Z 107$ & G004 \\
\hline $\mathrm{Z108}$ & G099 \\
\hline Z109 & G120 \\
\hline $\mathrm{Z110}$ & G039 \\
\hline Z111 & G210 \\
\hline$\overline{Z 112}$ & G086 \\
\hline Z113 & G161 \\
\hline Z114 & G182 \\
\hline 2115 & G063 \\
\hline 2116 & G192 \\
\hline 2117 & G174 \\
\hline$Z 118$ & G006 \\
\hline Z119 & G166 \\
\hline $\mathrm{Z120}$ & G163 \\
\hline 2121 & G054 \\
\hline Z122 & G095 \\
\hline$Z 123$ & G019 \\
\hline $\mathrm{Z124}$ & G205 \\
\hline 2125 & G175 \\
\hline Z126 & G094 \\
\hline Z127 & G216 \\
\hline $\mathrm{Z128}$ & G100 \\
\hline Z129 & G220 \\
\hline $\mathrm{Z130}$ & G104 \\
\hline $\mathrm{Z} 131$ & G052 \\
\hline$Z 132$ & G097 \\
\hline Z133 & G026 \\
\hline Z134 & G190 \\
\hline Z135 & G043 \\
\hline Z136 & G142 \\
\hline Z137 & G207 \\
\hline Z138 & G191 \\
\hline Z139 & G143 \\
\hline $\mathrm{Z} 140$ & G213 \\
\hline Z141 & G189 \\
\hline Z142 & G208 \\
\hline Z143 & G064 \\
\hline Z144 & G074 \\
\hline Z145 & G178 \\
\hline Z146 & $\mathrm{GO} 30$ \\
\hline$Z 147$ & G199 \\
\hline$Z 148$ & G200 \\
\hline Z149 & $\mathrm{G} 033$ \\
\hline $\mathrm{Z150}$ & G031 \\
\hline
\end{tabular}

\begin{tabular}{|c|c|}
\hline $\begin{array}{l}\text { Compact } \\
Z \text { Number }\end{array}$ & $\begin{array}{c}\text { Compact G } \\
\text { Number }\end{array}$ \\
\hline $\mathrm{Z} 151$ & G029 \\
\hline Z152 & G068 \\
\hline Z153 & G103 \\
\hline Z154 & G018 \\
\hline Z155 & G158 \\
\hline Z156 & $\mathrm{G} 023$ \\
\hline Z157 & G183 \\
\hline Z158 & G067 \\
\hline Z159 & G218 \\
\hline 2160 & G014 \\
\hline $\mathrm{Z} 161$ & G198 \\
\hline $\mathrm{Z} 162$ & G109 \\
\hline Z163 & G013 \\
\hline Z164 & $\mathrm{G} 044$ \\
\hline Z165 & G116 \\
\hline Z166 & G215 \\
\hline Z167 & G058 \\
\hline Z168 & G073 \\
\hline Z169 & G151 \\
\hline $\mathrm{Z} 170$ & G081 \\
\hline $\mathrm{Z171}$ & G015 \\
\hline Z172 & G119 \\
\hline Z173 & G181 \\
\hline $\mathrm{Z174}$ & G102 \\
\hline 2175 & G083 \\
\hline Z176 & G079 \\
\hline 2177 & G053 \\
\hline $\mathrm{Z} 178$ & G082 \\
\hline Z179 & G088 \\
\hline $\mathrm{Z180}$ & G136 \\
\hline Z181 & G105 \\
\hline $\mathrm{Z182}$ & G137 \\
\hline Z183 & G112 \\
\hline $\mathrm{Z} 184$ & G150 \\
\hline$Z 185$ & G159 \\
\hline 2186 & G173 \\
\hline Z187 & G196 \\
\hline Z188 & G027 \\
\hline Z189 & G113 \\
\hline Z190 & G165 \\
\hline 2191 & G134 \\
\hline Z192 & $\mathrm{G} 002$ \\
\hline 2193 & G131 \\
\hline Z194 & G146 \\
\hline 2195 & G115 \\
\hline 2196 & G186 \\
\hline Z197 & G016 \\
\hline Z198 & G184 \\
\hline Z199 & 6080 \\
\hline 2200 & G149 \\
\hline
\end{tabular}

\begin{tabular}{|c|c|}
\hline $\begin{array}{l}\text { Compact } \\
\text { Z Number }\end{array}$ & $\begin{array}{c}\text { Compact G } \\
\text { Number }\end{array}$ \\
\hline Z201 & G203 \\
\hline 2202 & G090 \\
\hline 2203 & G152 \\
\hline$Z 204$ & \\
\hline$Z 205$ & \\
\hline 2206 & \\
\hline 2207 & \\
\hline$Z 208$ & \\
\hline$Z 209$ & \\
\hline 2210 & \\
\hline 2211 & \\
\hline 2212 & \\
\hline 2213 & \\
\hline 2214 & \\
\hline$Z 215$ & \\
\hline 2216 & \\
\hline 2217 & \\
\hline 2218 & \\
\hline 2219 & \\
\hline 2220 & \\
\hline Z221 & \\
\hline $\mathrm{Z222}$ & \\
\hline 2223 & \\
\hline 2224 & \\
\hline 2225 & \\
\hline 2226 & \\
\hline 2227 & \\
\hline 2228 & \\
\hline 2229 & \\
\hline 2230 & \\
\hline 2231 & \\
\hline 2232 & \\
\hline 2233 & \\
\hline 2234 & \\
\hline 2235 & \\
\hline$Z 236$ & \\
\hline 2237 & \\
\hline 2238 & \\
\hline Z239 & \\
\hline$Z 240$ & \\
\hline 2241 & \\
\hline 2242 & \\
\hline 2243 & \\
\hline$Z 244$ & \\
\hline 2245 & \\
\hline 2246 & \\
\hline 2247 & \\
\hline 2248 & \\
\hline 2249 & \\
\hline 2250 & \\
\hline
\end{tabular}

Comments

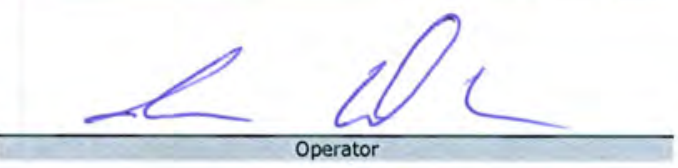

$$
3-10-10
$$


Data Report Form DRF-24D: Compact Charge Weight

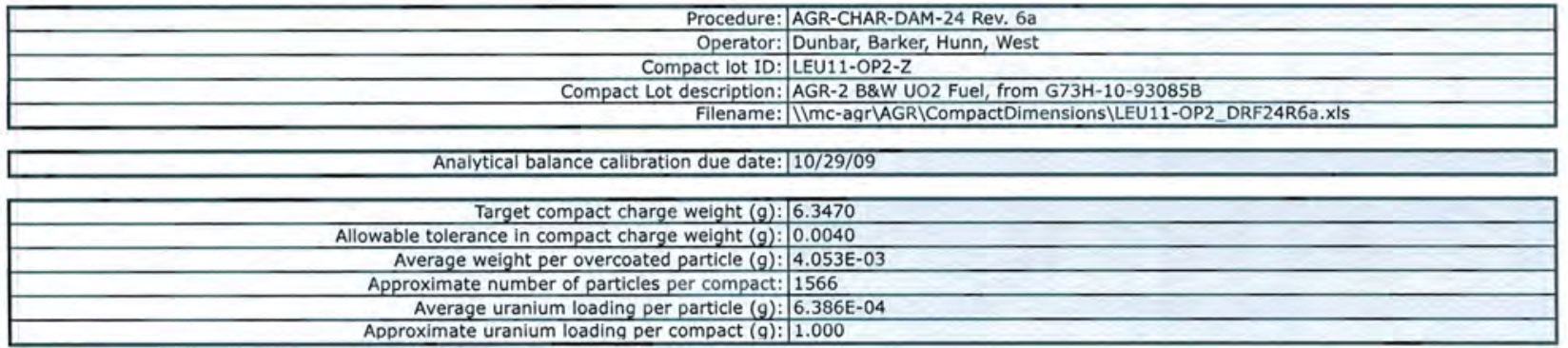

\begin{tabular}{|c|c|}
\hline $\begin{array}{c}\text { Compact } \\
\text { G Number }\end{array}$ & $\begin{array}{c}\text { Charge Weight } \\
(\mathrm{q})\end{array}$ \\
\hline $\mathrm{G} 001$ & 6.3478 \\
\hline $\mathrm{G} 002$ & 6.3463 \\
\hline $\mathrm{G} 003$ & 6.3475 \\
\hline $\mathrm{G} 004$ & 6.3465 \\
\hline $\mathrm{G} 005$ & 6.3475 \\
\hline $\mathrm{G} 006$ & 6.3462 \\
\hline $\mathrm{G} 007$ & 6.3467 \\
\hline $\mathrm{G} 008$ & 6.3475 \\
\hline $\mathrm{G} 009$ & 6.3472 \\
\hline $\mathrm{G} 010$ & 6.3486 \\
\hline $\mathrm{G} 011$ & 6.3481 \\
\hline $\mathrm{G} 012$ & 6.3460 \\
\hline $\mathrm{G} 013$ & 6.3462 \\
\hline $\mathrm{G} 014$ & 6.3460 \\
\hline $\mathrm{G} 015$ & 6.3490 \\
\hline $\mathrm{G} 016$ & 6.3461 \\
\hline $\mathrm{G} 017$ & 6.3444 \\
\hline $\mathrm{G} 018$ & 6.3467 \\
\hline $\mathrm{G} 019$ & 6.3470 \\
\hline $\mathrm{G} 020$ & 6.3468 \\
\hline $\mathrm{G} 021$ & 6.3462 \\
\hline $\mathrm{G} 022$ & 6.3484 \\
\hline $\mathrm{G} 023$ & 6.3487 \\
\hline $\mathrm{G} 024$ & 6.3493 \\
\hline $\mathrm{G} 025$ & 6.3489 \\
\hline $\mathrm{G} 026$ & 6.3467 \\
\hline $\mathrm{G} 027$ & 6.3470 \\
\hline $\mathrm{G} 028$ & 6.3483 \\
\hline $\mathrm{G} 029$ & 6.3449 \\
\hline $\mathrm{G} 030$ & 6.3439 \\
\hline $\mathrm{G} 031$ & 6.3499 \\
\hline $\mathrm{G} 032$ & 6.3447 \\
\hline $\mathrm{G} 033$ & 6.3438 \\
\hline $\mathrm{G} 034$ & 6.3490 \\
\hline $\mathrm{G} 035$ & 6.3470 \\
\hline $\mathrm{G} 036$ & 6.3470 \\
\hline $\mathrm{G} 037$ & 6.3488 \\
\hline $\mathrm{G} 038$ & 6.3496 \\
\hline $\mathrm{G} 039$ & 6.3489 \\
\hline $\mathrm{G} 040$ & 6.3466 \\
\hline $\mathrm{G} 041$ & 6.3488 \\
\hline $\mathrm{G} 042$ & 6.3466 \\
\hline $\mathrm{G} 043$ & 6.3484 \\
\hline $\mathrm{G} 044$ & 6.3461 \\
\hline $\mathrm{G} 045$ & 6.3496 \\
\hline $\mathrm{G} 046$ & 6.3476 \\
\hline & 6.3470 \\
\hline & 6.3478 \\
\hline & 6.3484 \\
\hline & 6.3482 \\
\hline
\end{tabular}

\begin{tabular}{|c|c|}
\hline $\begin{array}{l}\text { Compact } \\
\text { G Number }\end{array}$ & $\begin{array}{c}\text { Charge Weight } \\
\text { (a) }\end{array}$ \\
\hline G051 & 6.3454 \\
\hline G052 & 6.3464 \\
\hline G053 & 6.3475 \\
\hline G054 & 6.3487 \\
\hline G055 & 6.3449 \\
\hline G056 & 6.3453 \\
\hline G057 & 6.3453 \\
\hline G058 & 6.3487 \\
\hline G059 & 6.3482 \\
\hline G060 & 6.3474 \\
\hline G061 & 6.3487 \\
\hline G062 & 6.3449 \\
\hline G063 & 6.3476 \\
\hline G064 & 6.3454 \\
\hline G065 & 6.3499 \\
\hline G066 & 6.3453 \\
\hline G067 & 6.3452 \\
\hline G068 & 6.3455 \\
\hline G069 & 6.3465 \\
\hline G070 & 6.3466 \\
\hline G071 & 6.3471 \\
\hline G072 & 6.3464 \\
\hline G073 & 6.3489 \\
\hline G074 & 6.3446 \\
\hline G075 & 6.3461 \\
\hline G076 & 6.3458 \\
\hline G077 & 6.3492 \\
\hline G078 & 6.3460 \\
\hline G079 & 6.3479 \\
\hline G080 & 6.3470 \\
\hline G081 & 6.3486 \\
\hline G082 & 6.3465 \\
\hline G083 & 6.3464 \\
\hline G084 & 6.3477 \\
\hline G085 & 6.3509 \\
\hline G086 & 6.3462 \\
\hline G087 & 6.3495 \\
\hline G088 & 6.3459 \\
\hline G089 & 6.3441 \\
\hline G090 & 6.3470 \\
\hline G091 & 6.3459 \\
\hline G092 & 6.3492 \\
\hline G093 & 6.3461 \\
\hline G094 & 6.3457 \\
\hline G095 & 6.3485 \\
\hline G096 & 6.3497 \\
\hline G097 & 6.3448 \\
\hline G098 & 6.3480 \\
\hline G099 & 6.3477 \\
\hline G100 & 6.3442 \\
\hline
\end{tabular}

\begin{tabular}{|c|c|}
\hline $\begin{array}{c}\text { Compact } \\
\text { G Number }\end{array}$ & $\begin{array}{c}\text { Charge Weight } \\
\text { ( })\end{array}$ \\
\hline G101 & 6.3455 \\
\hline G102 & 6.3470 \\
\hline G103 & 6.3478 \\
\hline G104 & 6.3452 \\
\hline G105 & 6.3466 \\
\hline G106 & 6.3458 \\
\hline G107 & 6.3488 \\
\hline G108 & 6.3483 \\
\hline G109 & 6.3470 \\
\hline G110 & 6.3454 \\
\hline G111 & 6.3490 \\
\hline G112 & 6.3483 \\
\hline G113 & 6.3476 \\
\hline G114 & 6.3460 \\
\hline G115 & 6.3484 \\
\hline G116 & 6.3469 \\
\hline G117 & 6.3471 \\
\hline G118 & 6.3471 \\
\hline G119 & 6.3458 \\
\hline G120 & 6.3455 \\
\hline G121 & 6.3448 \\
\hline G122 & 6.3450 \\
\hline G123 & 6.3450 \\
\hline G124 & 6.3474 \\
\hline G125 & 6.3453 \\
\hline G126 & 6.3474 \\
\hline G127 & 6.3445 \\
\hline G128 & 6.3464 \\
\hline G129 & 6.3466 \\
\hline G130 & 6.3491 \\
\hline G131 & 6.3445 \\
\hline G132 & 6.3487 \\
\hline G133 & 6.3466 \\
\hline G134 & 6.3468 \\
\hline G135 & 6.3458 \\
\hline G136 & 6.3487 \\
\hline G137 & 6.3499 \\
\hline G138 & 6.3464 \\
\hline G139 & 6.3472 \\
\hline G140 & 6.3458 \\
\hline G141 & 6.3448 \\
\hline G142 & 6.3477 \\
\hline G143 & 6.3459 \\
\hline G144 & 6.3465 \\
\hline G145 & 6.3468 \\
\hline G146 & 6.3485 \\
\hline G148 & 6.3483 \\
\hline & 6.3484 \\
\hline & 6.3479 \\
\hline
\end{tabular}

\begin{tabular}{|c|c|}
\hline $\begin{array}{c}\text { Compact } \\
\text { G Number }\end{array}$ & $\begin{array}{c}\text { Charge Weigh } \\
\text { (q) }\end{array}$ \\
\hline G151 & 6.3463 \\
\hline G152 & 6.3462 \\
\hline G153 & 6.3494 \\
\hline G154 & 6.3479 \\
\hline G155 & 6.3488 \\
\hline G156 & 6.3479 \\
\hline G157 & 6.3449 \\
\hline G158 & 6.3450 \\
\hline G159 & 6.3471 \\
\hline G160 & 6.3462 \\
\hline G161 & 6.3460 \\
\hline G162 & 6.3462 \\
\hline G163 & 6.3470 \\
\hline G164 & 6.3474 \\
\hline G165 & 6.3477 \\
\hline G166 & 6.3473 \\
\hline G167 & 6.3475 \\
\hline G168 & 6.3438 \\
\hline G169 & 6.3470 \\
\hline G170 & 6.3464 \\
\hline G171 & 6.3491 \\
\hline G172 & 6.3451 \\
\hline G173 & 6.3474 \\
\hline G174 & 6.3473 \\
\hline G175 & 6.3448 \\
\hline G176 & 6.3456 \\
\hline G177 & 6.3448 \\
\hline G178 & 6.3463 \\
\hline G179 & 6.3479 \\
\hline G180 & 6.3446 \\
\hline G181 & 6.3488 \\
\hline G182 & 6.3457 \\
\hline G183 & 6.3491 \\
\hline G184 & 6.3493 \\
\hline G185 & 6.3474 \\
\hline G186 & 6.3465 \\
\hline G187 & 6.3472 \\
\hline G188 & 6.3497 \\
\hline G189 & 6.3496 \\
\hline G190 & 6.3486 \\
\hline G191 & 6.3444 \\
\hline G192 & 6.3470 \\
\hline G193 & 6.3458 \\
\hline G194 & 6.3463 \\
\hline G195 & 6.3453 \\
\hline G196 & 6.3448 \\
\hline G197 & 6.3448 \\
\hline G198 & 6.3490 \\
\hline G200 & 6.3484 \\
\hline & 6.3470 \\
\hline
\end{tabular}

\begin{tabular}{|c|c|}
\hline $\begin{array}{l}\text { Compact } \\
\text { G Number }\end{array}$ & $\begin{array}{c}\text { Charge } \\
\text { Weight (q) }\end{array}$ \\
\hline G201 & 6.3477 \\
\hline G202 & 6.3490 \\
\hline G203 & 6.3493 \\
\hline G204 & 6.3466 \\
\hline G205 & 6.3479 \\
\hline G206 & 6.3474 \\
\hline G207 & 6.3464 \\
\hline G208 & 6.3457 \\
\hline G209 & 6.3498 \\
\hline G210 & 6.3474 \\
\hline G211 & 6.3459 \\
\hline G212 & 6.3504 \\
\hline G213 & 6.3510 \\
\hline G214 & 6.3451 \\
\hline G215 & 6.3447 \\
\hline G216 & 6.3438 \\
\hline G217 & 6.3464 \\
\hline G218 & 6.3460 \\
\hline G219 & 6.3481 \\
\hline $\mathrm{G} 220$ & 6.3490 \\
\hline G221 & \\
\hline G222 & \\
\hline G223 & \\
\hline G224 & \\
\hline G225 & \\
\hline G226 & \\
\hline G227 & \\
\hline G228 & \\
\hline G229 & \\
\hline G230 & \\
\hline G231 & \\
\hline G232 & \\
\hline G233 & \\
\hline G234 & \\
\hline G235 & \\
\hline G236 & \\
\hline G237 & \\
\hline G238 & \\
\hline G239 & \\
\hline G240 & \\
\hline G241 & \\
\hline G242 & \\
\hline G243 & \\
\hline G244 & \\
\hline G245 & \\
\hline G246 & \\
\hline G247 & \\
\hline G248 & \\
\hline G249 & \\
\hline G250 & \\
\hline
\end{tabular}

Comments

Average weight per overcoated particle from combined results of 2 independent measurements (W09081401 and W09081402).
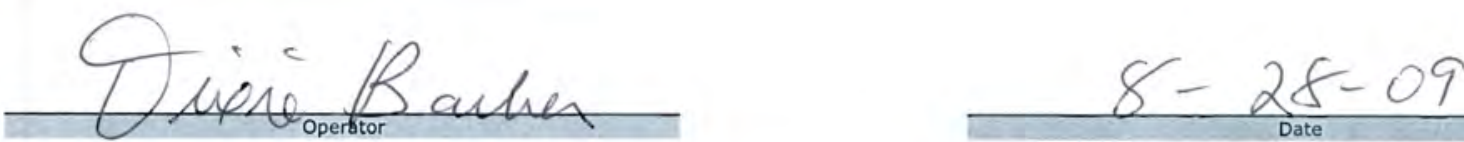


\section{Appendix B : Particle diameter, weight and volume}

To measure particle diameter, particles are placed in a tray in a single layer. A microscope system is set up to obtain a shadow image of each particle in the tray. A series of image frames are recorded to cover the area of the tray in a tiled array. The images are saved on a server for subsequent processing to extract the edge coordinates at multiple points around the perimeter. The mean diameter is then calculated from the edge coordinates. DRF-10A shows the distribution of the mean diameter measured on each particle and calculates the average and standard deviation for the entire sample.

Average particle envelope volume is measured on a sample of a few thousand particles using a mercury porosimeter. Particles are placed in an evacuated cell, which is back-filled with mercury. Pressure is applied to force the mercury into the interparticle void space until each particle is individually enveloped by mercury. The weight of mercury displaced by the particles is then measured and a total particle envelope volume is calculated. The number of particles in the sample is calculated from the sample weight divided by the average particle weight. Riffling five representative random samples, then weighing and counting each sample, is used to determine average particle weight. Average envelope volume per particle is calculated by dividing total particle envelope volume by the number of particles in the sample. DRF-22 shows the results of the particle weight analysis and DRF-31 reports the average particle envelope volume, as well as the envelope density and open porosity. The open porosity of a sample of particles is defined as the total open pore volume of the sample divided by the total surface area of the sample. Open pore volume is determined by measuring the mercury intrusion into the sample over a specified pressure range, normally between 250 and 10,000 psia. Surface area is approximated by calculating the average particle diameter from the envelope volume of the sample and the number of particles in the sample (assuming each particle is a perfect sphere). 


\section{Data Report Form DRF-10A: Measurement of Particle Diameter}

\begin{tabular}{|c|c|}
\hline Procedure: & AGR-CHAR-DAM-10 Rev. 2 \\
\hline Operator: & Andrew K. Kercher \\
\hline Folder name containing images: & 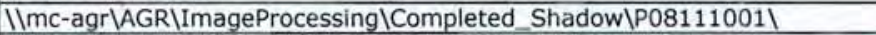 \\
\hline Sample ID: & LEU06-B01 \\
\hline Sample Description: & AGR-2 UCO Variant 1, G73]-14-93074A \\
\hline Folder name containing processed data: & 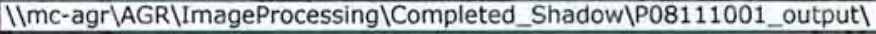 \\
\hline
\end{tabular}

Number of particles analyzed: $\quad 2002$

Mean of the average diameter of each particle ( $\mu \mathrm{m})$ :

Standard deviation in the average diameter of each particle $(\mu \mathrm{m})$ :

874.7

Distribution of the average particle diameter (top binned)

\begin{tabular}{|c|c|}
\hline Mean Diameter $(\mu \mathrm{m})$ & Frequency \\
\hline \hline$<800$ & 0 \\
\hline 810 & 2 \\
\hline 820 & 1 \\
\hline 830 & 37 \\
\hline 840 & 66 \\
\hline 850 & 179 \\
\hline 860 & 254 \\
\hline 870 & 313 \\
\hline 880 & 356 \\
\hline 890 & 300 \\
\hline 900 & 232 \\
\hline 910 & 137 \\
\hline 920 & 71 \\
\hline 930 & 37 \\
\hline 940 & 13 \\
\hline 950 & 4 \\
\hline$>950$ & 0 \\
\hline
\end{tabular}
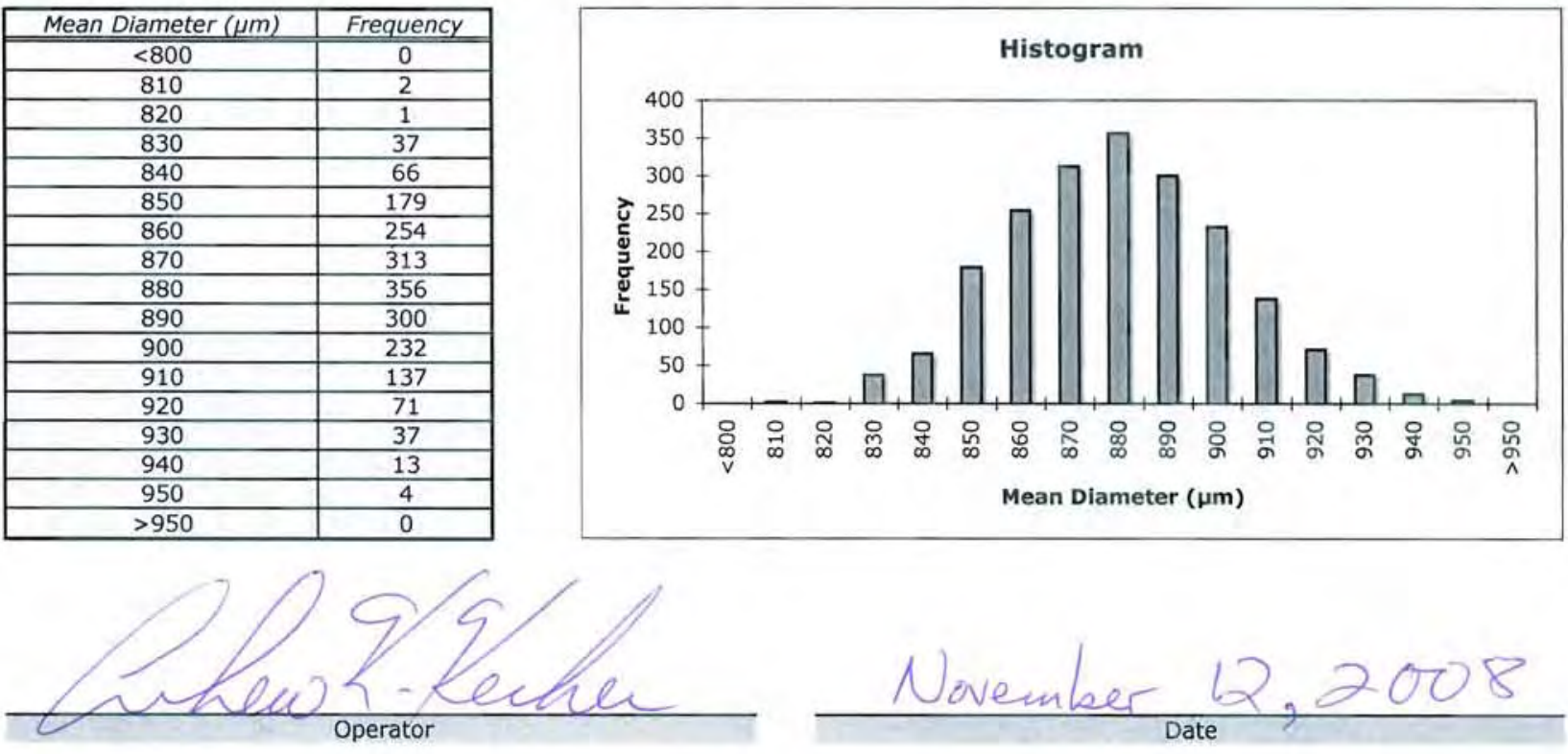
Data Report Form DRF-22: Estimation of Average Particle Weight

\begin{tabular}{|c|c|c|c|c|c|}
\hline & Procedure: & \multicolumn{4}{|c|}{ AGR-CHAR-DAM-22 Rev. 1} \\
\hline & Operator: & \multicolumn{4}{|c|}{ Dixie Barker } \\
\hline & Particle Lot ID: & \multicolumn{4}{|l|}{ LEU06-C01 } \\
\hline \multicolumn{2}{|c|}{ Particle Lot Description: } & \multicolumn{4}{|c|}{ AGR-2 UCO Variant 1, G73J-14-93074A } \\
\hline & Filename: & \multicolumn{4}{|c|}{ IImc-agr\AGR\ParticleWeight|W08102001_DRF22R1.xIs } \\
\hline & Sample 1 & Sample 2 & Sample 3 & Sample 4 & Sample 5 \\
\hline Weight of particles $(g)$ : & 0.1808 & 0.2720 & 0.3211 & 0.2699 & 0.2992 \\
\hline Number of particles: & 175 & 262 & 310 & 260 & 289 \\
\hline Average weight/particle $(\mathrm{g}):$ & $1.033 \mathrm{E}-03$ & $1.038 \mathrm{E}-03$ & $1.036 \mathrm{E}-03$ & $1.038 \mathrm{E}-03$ & $1.035 \mathrm{E}-03$ \\
\hline
\end{tabular}

Mean average weight/particle $(\mathrm{g}): 1.036 \mathrm{E}-03$

Standard error in mean average weight/particle $(\mathrm{g}): 9.40 \mathrm{E}-07$
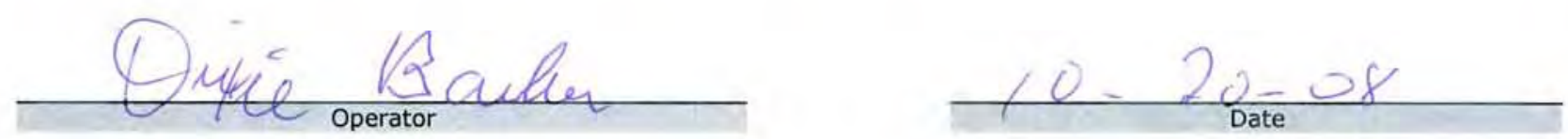
Data Report Form DRF-31: Measurement of Open Porosity using a Mercury Porosimeter

\begin{tabular}{|c|c|}
\hline Procedure: & AGR-CHAR-DAM-31 Rev. 1 \\
\hline Operator: & S. D. Nunn \\
\hline Coated particle batch ID: & LEU06-B01 \\
\hline Batch Description: & AGR-2 UCO Variant, G73J-14-93074A \\
\hline Thermocouple Expiration Date: & $5 / 15 / 09$ \\
\hline Penetrometer Expiration Date: & $7 / 10 / 09$ \\
\hline Completed DRF Filename: & IImc-agr|AGR\Porosimeter|S08120401/S08120401_DRF31R1.xIs \\
\hline
\end{tabular}

Mean average weight/particle $(\mathrm{g}): 1.04 \mathrm{E}-03$

Standard error in mean average weight/particle $(\mathrm{g}): 19.40 \mathrm{E}-07$

\begin{tabular}{|r|l|}
\hline Weight of particles $(\mathrm{g}):$ & 3.8494 \\
\hline Approximate number of particles: & 3716 \\
\hline Uncertainty in number of particles: & 3 \\
\hline Total envelope volume of sample $(\mathrm{cc}):$ & 1.284 \\
\hline Average envelope volume/particle $(\mathrm{cc}):$ & $3.46 \mathrm{E}-04$ \\
\hline Sample envelope density $(\mathrm{g} / \mathrm{cc}):$ & 2.998 \\
\hline
\end{tabular}

Average particle diameter (microns): $8.71 \mathrm{E}+02$

Average surface area/particle $(\mathrm{cm} 2): 2.38 \mathrm{E}-02$

Total sample surface area $(\mathrm{cm} 2): 8.85 \mathrm{E}+01$

Intruded mercury volume from $250-10,000 \mathrm{psia}(\mathrm{cc}): 1.90 \mathrm{E}-03$

Open porosity $(\mathrm{ml} / \mathrm{m} 2): 2.15 \mathrm{E}-01$

\section{Comments}

Sample LEU06-B01 was used after particle size and shape analysis was complete because LEU06-D01 was not available.
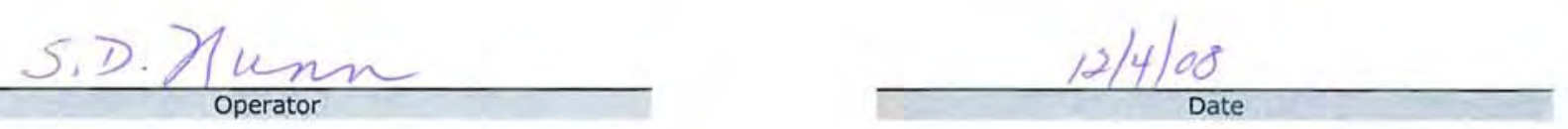


\section{Data Report Form DRF-10A: Measurement of Particle Diameter}

\begin{tabular}{|c|c|}
\hline Procedure: & AGR-CHAR-DAM-10 Rev. 2 \\
\hline Operator: & Andrew K. Kercher \\
\hline Folder name containing images: & 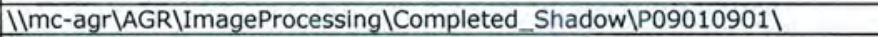 \\
\hline Sample ID: & LEU07-B01 \\
\hline Sample Description: & AGR-2 UCO Baseline, from G73]-14-93072A \\
\hline Folder name containing processed data: & 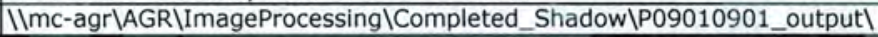 \\
\hline
\end{tabular}

Number of particles analyzed:

Mean of the average diameter of each particle $(\mu \mathrm{m})$ :

Standard deviation in the average diameter of each particle $(\mu \mathrm{m})$ :

2174

Distribution of the average particle diameter (top binned)

\begin{tabular}{|c|c|}
\hline Mean Diameter $(\mu \mathrm{m})$ & Frequency \\
\hline \hline$<790$ & 0 \\
\hline 800 & 4 \\
\hline 810 & 23 \\
\hline 820 & 44 \\
\hline 830 & 121 \\
\hline 840 & 209 \\
\hline 850 & 269 \\
\hline 860 & 377 \\
\hline 870 & 364 \\
\hline 880 & 279 \\
\hline 890 & 216 \\
\hline 900 & 138 \\
\hline 910 & 76 \\
\hline 920 & 39 \\
\hline 930 & 12 \\
\hline 940 & 3 \\
\hline$>940$ & 0 \\
\hline
\end{tabular}
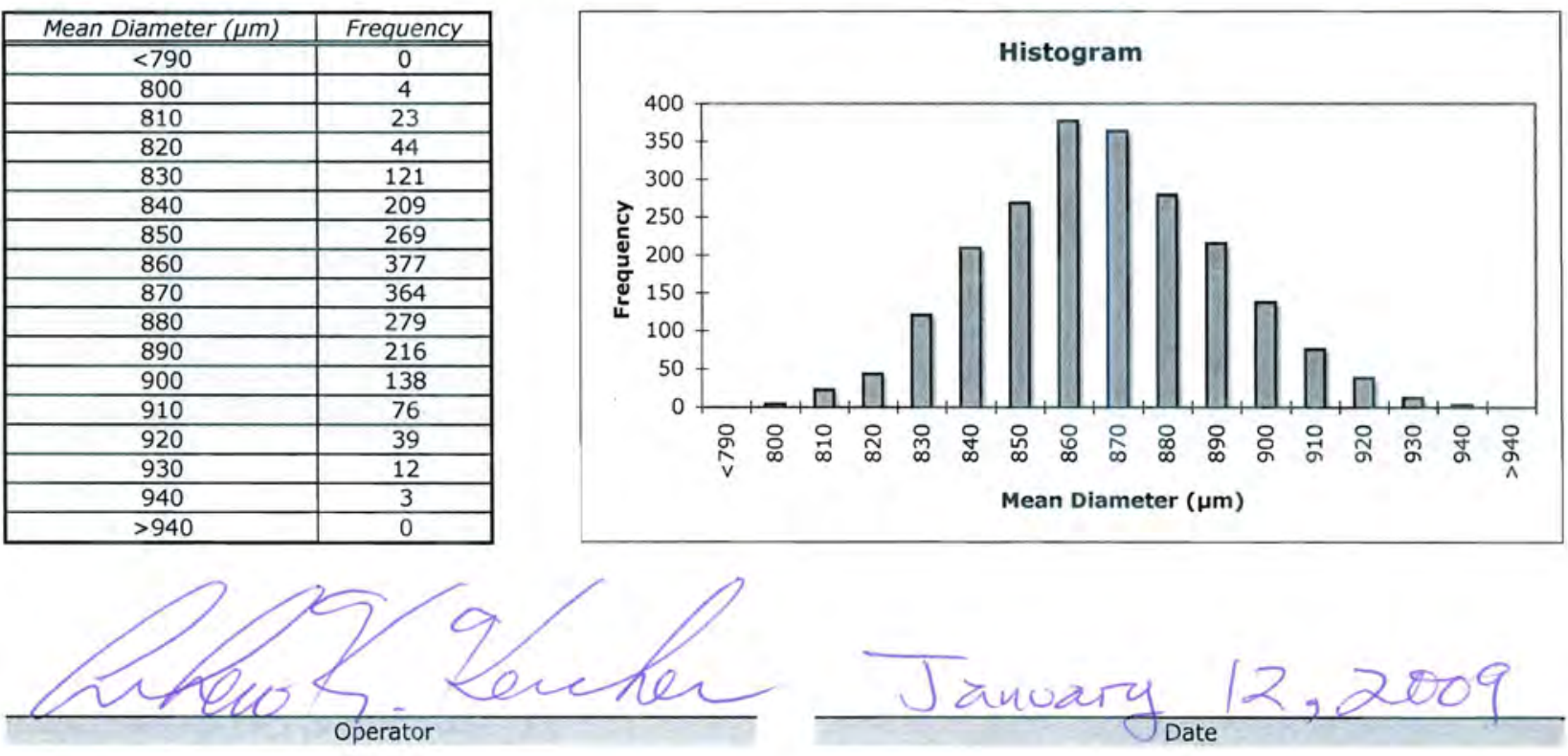
Data Report Form DRF-22: Estimation of Average Particle Weight

\begin{tabular}{|r|l|}
\hline Procedure: & AGR-CHAR-DAM-22 Rev. 1 \\
\hline Operator: & Dixie Barker \\
\hline Particle Lot ID: & LEU07 \\
\hline Particle Lot Description: & AGR-2 UCO Baseline, from G73J-14-93072A \\
\hline Filename: & \ImC-agr\AGR\ParticleWeight \W09010801_DRF22R1.xIs \\
\hline
\end{tabular}

\begin{tabular}{|r|c|c|c|c|c|}
\hline & Sample 1 & Sample 2 & Sample 3 & Sample 4 & Sample 5 \\
\hline Weight of particles (g): & 0.1174 & 0.1920 & 0.2865 & 0.3047 & 0.2650 \\
\hline Number of particles: & 118 & 191 & 284 & 303 & 265 \\
\hline Average weight/particle (g): & $9.949 \mathrm{E}-04$ & $1.005 \mathrm{E}-03$ & $1.009 \mathrm{E}-03$ & $1.006 \mathrm{E}-03$ & $1.000 \mathrm{E}-03$ \\
\hline
\end{tabular}

Mean average weight/particle (g): $1.003 \mathrm{E}-03$

Standard error in mean average weight/particle $(\mathrm{g}): 2.45 \mathrm{E}-06$
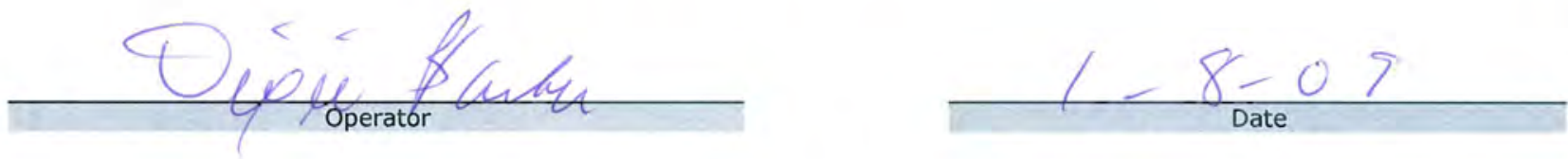
Data Report Form DRF-22: Estimation of Average Particle Weight

\begin{tabular}{|c|c|c|c|c|c|}
\hline & Procedure: & \multicolumn{4}{|c|}{ AGR-CHAR-DAM-22 Rev. 1} \\
\hline & Operator: & \multicolumn{4}{|c|}{ Dixie Barker } \\
\hline & Particle Lot ID: & \multicolumn{4}{|l|}{ LEU07 } \\
\hline Particle & Lot Description: & \multicolumn{4}{|c|}{ AGR-2 UCO Baseline, from G73J-14-93072A } \\
\hline & Filename: & \multicolumn{4}{|c|}{ \Imc-agr|AGR\ParticleWeight|W09011403_DRF22R1.xls } \\
\hline & Sample 6 & Sample 7 & Sample 8 & Sample 9 & Sample 10 \\
\hline Weight of particles $(\mathrm{g})$ : & 0.2836 & 0.1488 & 0.1596 & 0.1329 & 0.1461 \\
\hline Number of particles: & 282 & 148 & 160 & 132 & 145 \\
\hline Average weight/particle $(\mathrm{g})$ : & $1.006 \mathrm{E}-03$ & $1.005 \mathrm{E}-03$ & 9.975E-04 & 1.007E-03 & $1.008 \mathrm{E}-03$ \\
\hline
\end{tabular}

Mean average weight/particle $(\mathrm{g}): 1.005 \mathrm{E}-03$

Standard error in mean average weight/particle $(\mathrm{g}): 1.82 \mathrm{E}-06$
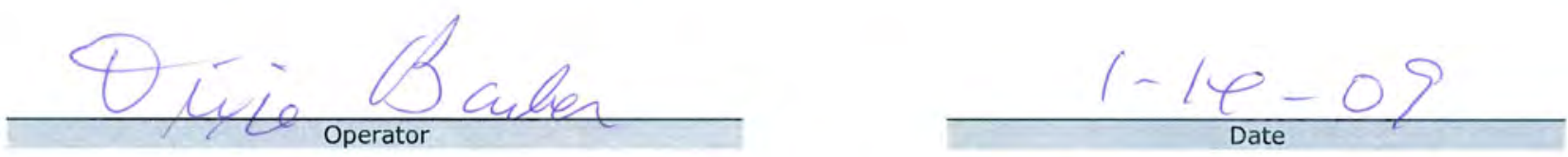
Data Report Form DRF-31: Measurement of Open Porosity using a Mercury Porosimeter

\begin{tabular}{|c|c|}
\hline Procedure: & AGR-CHAR-DAM-31 Rev. 1 \\
\hline Operator: & S. D. Nunn \\
\hline Coated particle batch ID: & LEU07-D01 \\
\hline Batch Description: & AGR-2 UCO Baseline, from G73J-14-93072A \\
\hline Thermocouple Expiration Date: & $5 / 15 / 09$ \\
\hline Penetrometer Expiration Date: & $7 / 10 / 09$ \\
\hline Completed DRF Filename: & IImc-agr|AGR\Porosimeter|S09012201\S09012201_DRF31R1.xls \\
\hline
\end{tabular}

Mean average weight/particle $(g): 1.004 \mathrm{E}-03$

Standard error in mean average weight/particle $(\mathrm{g}): 1.5 \mathrm{E}-06$

\begin{tabular}{|r|l|}
\hline Weight of particles $(\mathrm{g}):$ & 3.9258 \\
\hline Approximate number of particles: & 3910 \\
\hline Uncertainty in number of particles: & 6 \\
\hline Total envelope volume of sample $(\mathrm{cc}):$ & 1.285 \\
\hline Average envelope volume/particle $(\mathrm{cc}):$ & $3.29 \mathrm{E}-04$ \\
\hline Sample envelope density $(\mathrm{g} / \mathrm{cc}):$ & 3.055 \\
\hline &
\end{tabular}

\begin{tabular}{|r|l|}
\hline Average particle diameter (microns): & $8.56 \mathrm{E}+02$ \\
\hline Average surface area/particle $(\mathrm{cm} 2):$ & $2.30 \mathrm{E}-02$ \\
\hline Total sample surface area $(\mathrm{cm} 2):$ & $9.01 \mathrm{E}+01$ \\
\hline Open porosity $(\mathrm{ml} / \mathrm{m} 2):$ & $3.60 \mathrm{E}-03$ \\
\hline Intruded mercury volume from $250-10,000 \mathrm{psi}(\mathrm{cc}-01$ \\
\hline
\end{tabular}


Data Report Form DRF-10A: Measurement of Particle Diameter

\begin{tabular}{|c|c|}
\hline Procedure: & AGR-CHAR-DAM-10 Rev. 2 \\
\hline Operator: & Andrew K. Kercher \\
\hline Folder name containing images: & 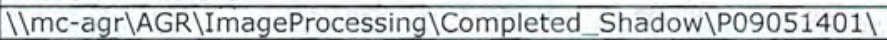 \\
\hline Sample ID: & LEU09-B01 \\
\hline Sample Description: & AGR-2 UCO Variant, from G73]-14-93073A \\
\hline
\end{tabular}

\begin{tabular}{rc|}
\hline Number of particles analyzed: & 2003 \\
\hline Mean of the average diameter of each particle $(\mu \mathrm{m})$ & 873.2 \\
\hline Standard deviation in the average diameter of each particle $(\mu \mathrm{m})$ & 23 \\
\hline
\end{tabular}

Distribution of the average particle diameter (top binned)

\begin{tabular}{|c|c|}
\hline Mean Diameter $(\mu \mathrm{m})$ & Frequency \\
\hline \hline$<800$ & 0 \\
\hline 810 & 2 \\
\hline 820 & 7 \\
\hline 830 & 35 \\
\hline 840 & 122 \\
\hline 850 & 160 \\
\hline 860 & 279 \\
\hline 870 & 295 \\
\hline 880 & 338 \\
\hline 890 & 285 \\
\hline 900 & 227 \\
\hline 910 & 135 \\
\hline 920 & 66 \\
\hline 930 & 28 \\
\hline 940 & 15 \\
\hline 950 & 8 \\
\hline$>950$ & 1 \\
\hline
\end{tabular}
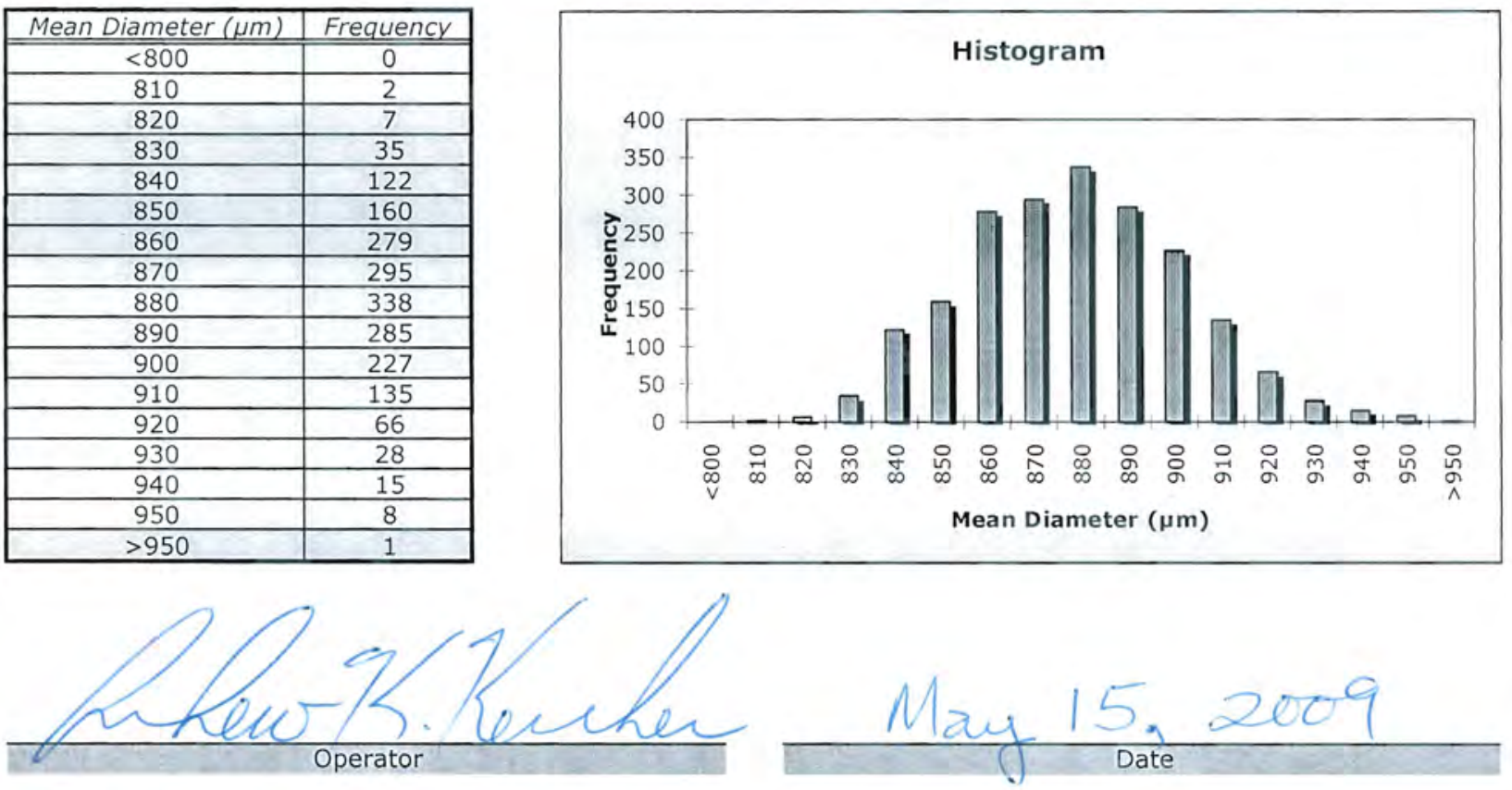
ORNL/TM-2010/296

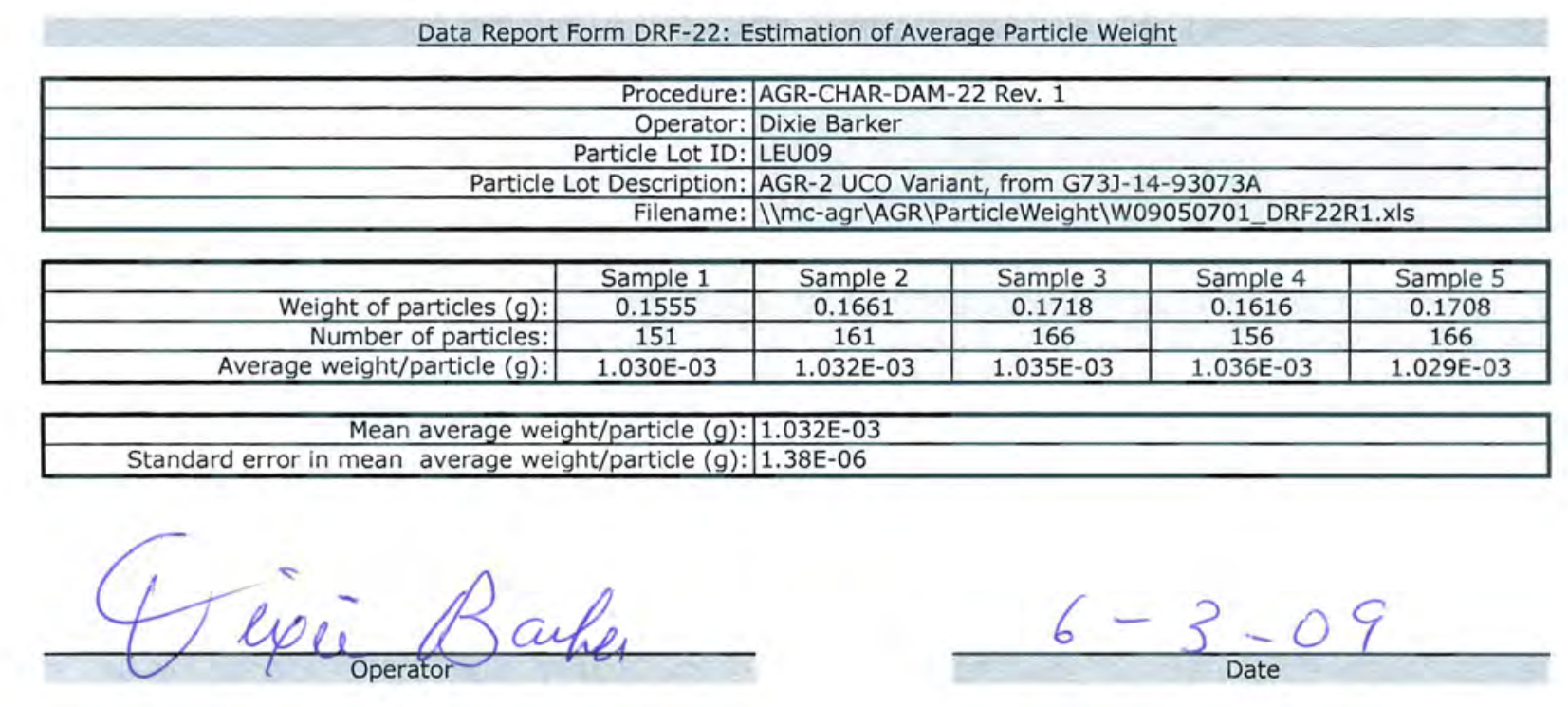




\section{Data Report Form DRF-31: Measurement of Open Porosity using a Mercury Porosimeter}

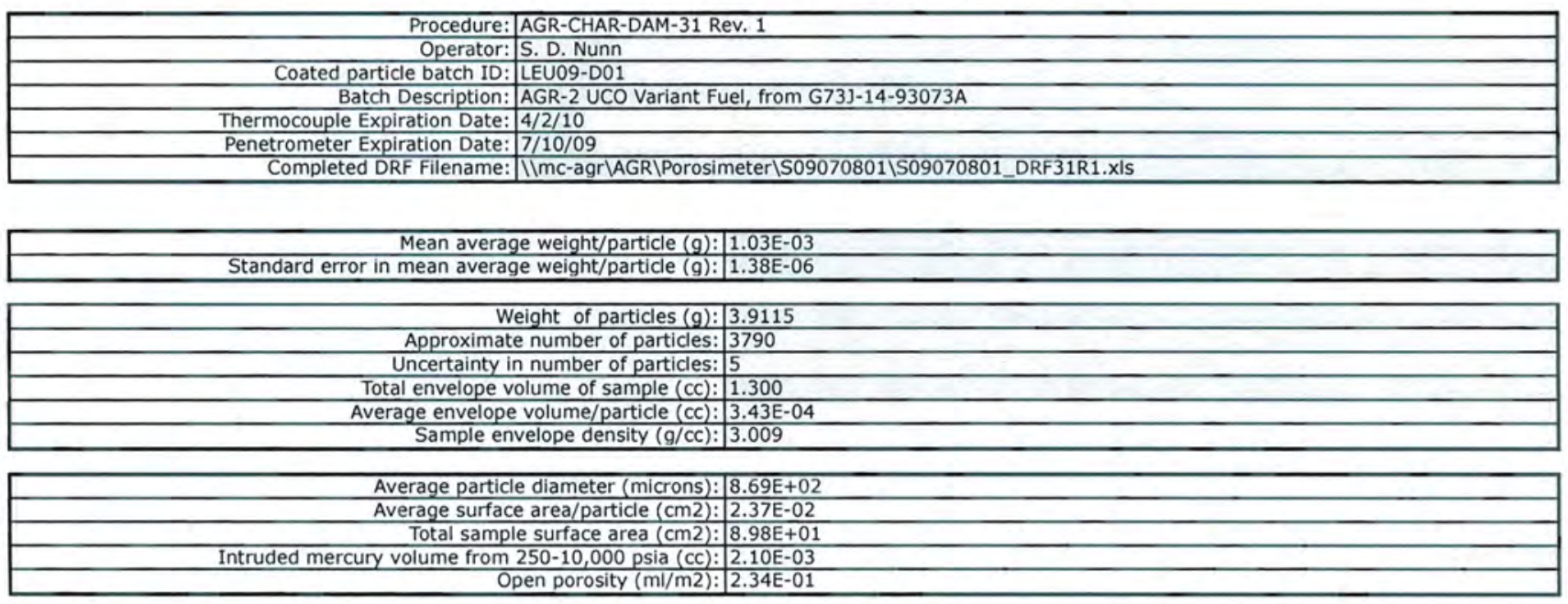


Data Report Form DRF-10A: Measurement of Particle Diameter

Procedure: AGR-CHAR-DAM-10 Rev. 2

Operator: Andrew K. Kercher

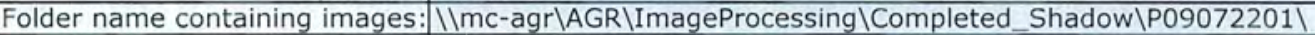

Sample ID: LEU11-B01

Sample Description: AGR-2 B\&W UO2 Fuel, from G73H-10-93085B

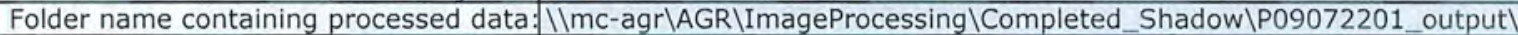

\begin{tabular}{|r|c|}
\hline Number of particles analyzed: & 1424 \\
\hline Mean of the average diameter of each particle $(\mu \mathrm{m})$ & 953.0 \\
\hline Standard deviation in the average diameter of each particle $(\mu \mathrm{m})$ & 28 \\
\hline
\end{tabular}

\section{Distribution of the average particle diameter (top binned)}

\begin{tabular}{|c|c|}
\hline Mean Diameter $(\mu \mathrm{m})$ & Frequency \\
\hline \hline$<880$ & 7 \\
\hline 890 & 13 \\
\hline 900 & 29 \\
\hline 910 & 46 \\
\hline 920 & 78 \\
\hline 930 & 119 \\
\hline 940 & 171 \\
\hline 950 & 193 \\
\hline 960 & 188 \\
\hline 970 & 201 \\
\hline 980 & 146 \\
\hline 990 & 97 \\
\hline 1000 & 64 \\
\hline 1010 & 35 \\
\hline 1020 & 23 \\
\hline 1030 & 9 \\
\hline$>1030$ & 5 \\
\hline
\end{tabular}
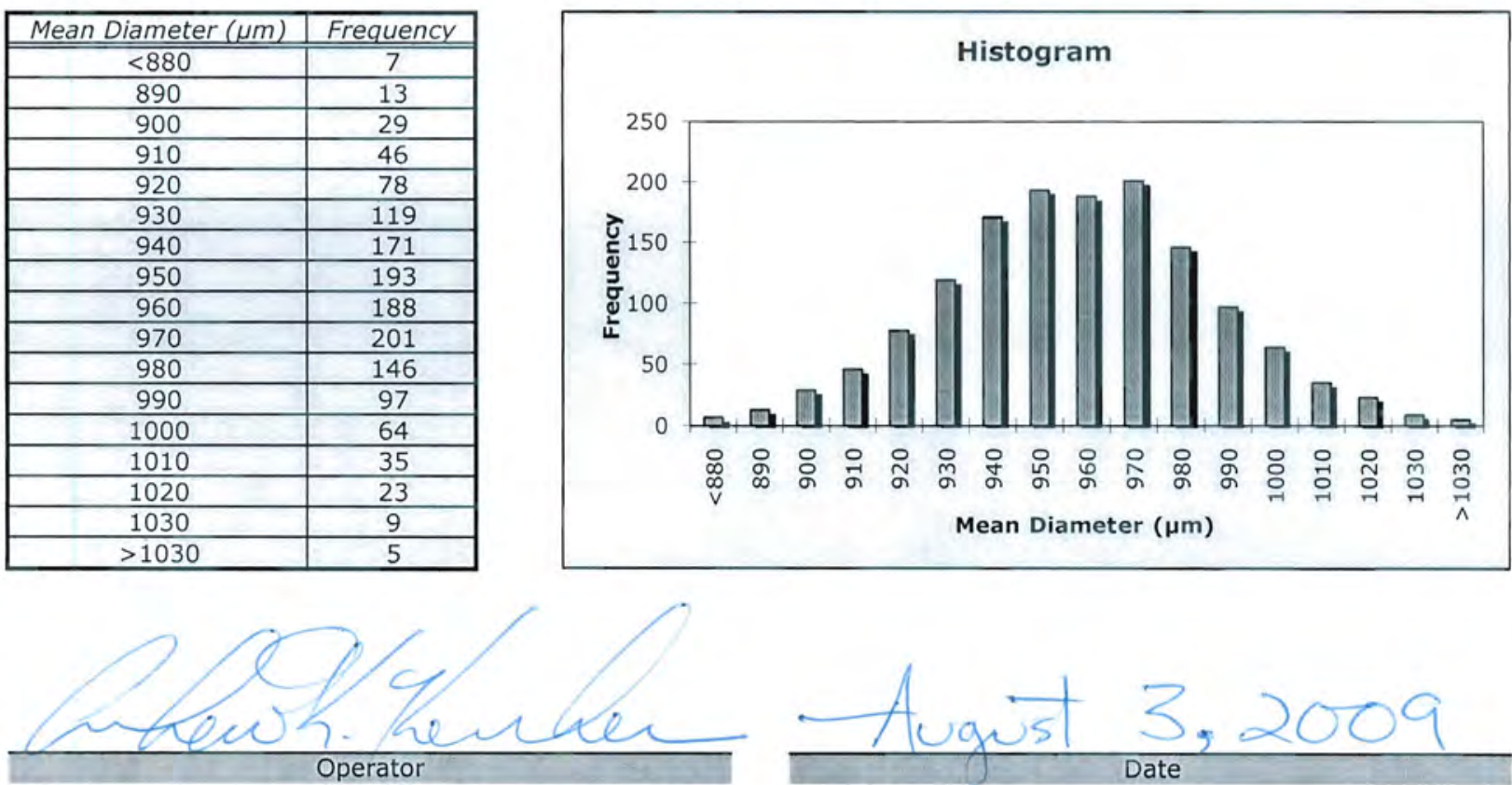
ORNL/TM-2010/296

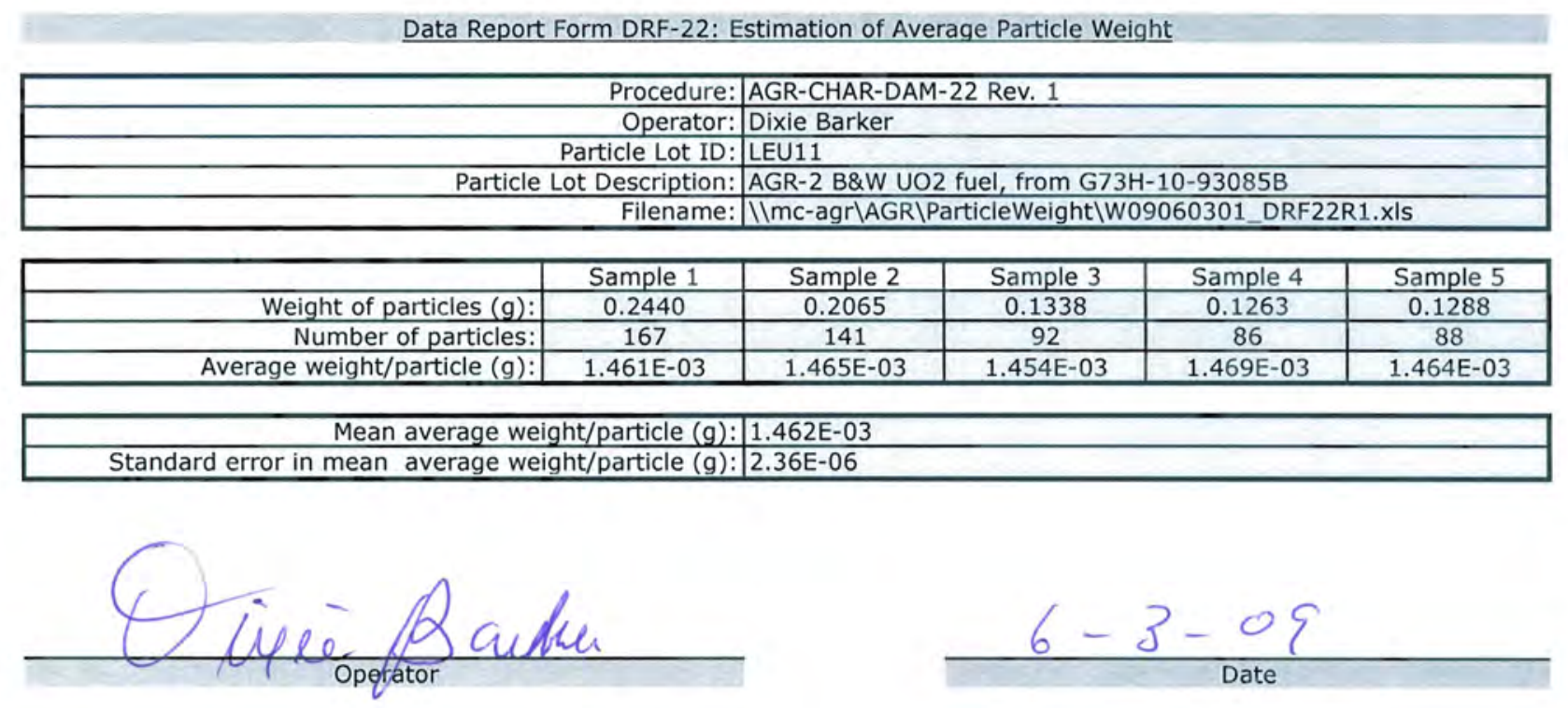


Data Report Form DRF-31: Measurement of Open Porosity using a Mercury Porosimeter

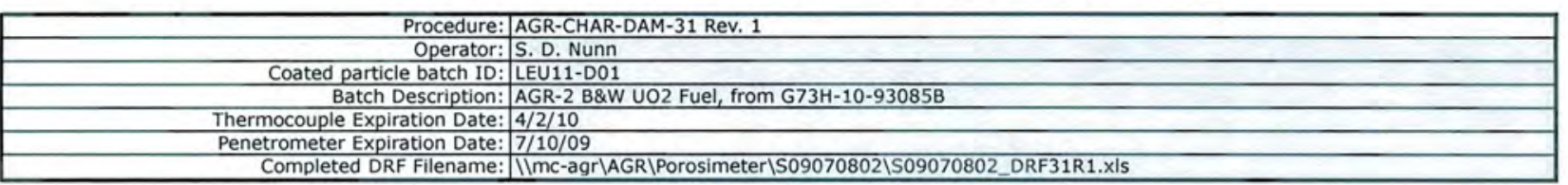

Mean average weight/particle (g): $1.46 \mathrm{E}-03$

\begin{aligned} & Mean average weight/particle $(\mathrm{g}): 1.46 \mathrm{E}-03 \\ &$ Standard error in mean average weight/particle $(\mathrm{g}): 2.36 \mathrm{E}-06 \\ &$\hline\end{aligned}

\begin{tabular}{|r|l|}
\hline Weight of particles $(\mathrm{g}):$ & 3.8057 \\
\hline Approximate number of particles: & 2603 \\
\hline Uncertainty in number of particles: & 4 \\
\hline Total envelope volume of sample (cc): & 1.157 \\
\hline Average envelope volume/particle $(\mathrm{cc}):$ & $4.45 \mathrm{E}-04$ \\
\hline Sample envelope density $(\mathrm{g} / \mathrm{cc}):$ & 3.288 \\
\hline
\end{tabular}

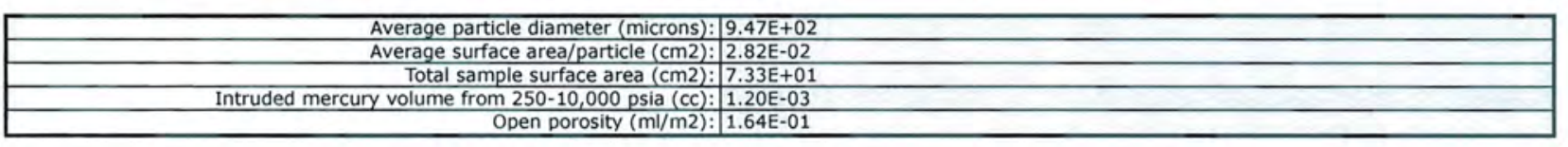
S.D.Xun
$7 / 8 / 09$

Date 


\section{Appendix C : Particle and compact uranium loading}

To measure the average uranium loading in the fuel particles, particles are heated at $900^{\circ} \mathrm{C}$ in air to burn off the OPyC. The residual SiC-coated particles are milled to a fine powder, exposing the uranium in the kernels. The powder is heated in air at $750^{\circ} \mathrm{C}$ to burn off the remaining pyrocarbon from the IPyC and buffer coating layers and to oxidize the uranium to $\mathrm{U}_{3} \mathrm{O}_{8}$. The residue is leached with concentrated nitric acid to dissolve the uranium oxide. The nitric acid leachate is sent to an independent analytical chemistry laboratory for measurement of the total uranium content using the Davies-Gray titration method. The number of particles in the sample is calculated from the sample weight divided by the average particle weight, and the total uranium in the sample is divided by the number of particles in the sample to get an average uranium loading per particle. DRF35 shows the results of this analysis on three samples and calculates a mean and standard deviation from these three analyses.

To measure the average uranium loading in the fuel compacts, a compact is heated at $900^{\circ} \mathrm{C}$ in air to burn off compact matrix and the OPyC. The residual SiC-coated particles are milled to a fine powder, exposing the uranium in the kernels. The powder is heated in air at $750^{\circ} \mathrm{C}$ to burn off the remaining carbon from the IPyC and buffer coating layers and to oxidize the uranium to $\mathrm{U}_{3} \mathrm{O}_{8}$. The residue is leached with concentrated nitric acid to dissolve the uranium oxide. The nitric acid leachate is diluted and sent to an independent analytical chemistry laboratory for measurement of the total uranium content using the Davies-Gray titration method. DRF-25 shows the results of this analysis on six compacts and calculates a mean and standard deviation from these six analyses. 
Data Report Form DRF-35: Fuel Particle Uranium Loading

\begin{tabular}{|c|c|c|c|c|c|c|}
\hline Procedure: & \multicolumn{6}{|c|}{ AGR-CHAR-DAM-35 Rev. 0} \\
\hline Operator: & \multicolumn{6}{|c|}{ Fred Montgomery } \\
\hline Particle lot ID: & \multicolumn{6}{|c|}{ LEU-06 } \\
\hline Particle lot description: & \multicolumn{6}{|c|}{ AGR-2 UCO Variant, from G73J-14-93074A } \\
\hline Filename: & \multicolumn{6}{|c|}{ IImc-agrVAGRIUraniumLoading|LEU06_DRF35R0.xIs } \\
\hline \multicolumn{7}{|c|}{ Mean average weight per particle $(g): 1.036 \mathrm{E}-03$} \\
\hline \multicolumn{7}{|c|}{ Standard error in mean average weight per particle $(\mathrm{g}): 9.4 \mathrm{E}-07$} \\
\hline & \multicolumn{2}{|c|}{ Sample 1 } & \multicolumn{2}{|c|}{ Sample 2 } & \multicolumn{2}{|c|}{ Sample 3} \\
\hline & Leach 1 & Leach 2 & Leach 1 & Leach 2 & Leach 1 & Leach 2 \\
\hline Particle sample ID: & \multicolumn{2}{|c|}{ LEU06-E01 } & \multicolumn{2}{|c|}{ LEU06-F01 } & \multicolumn{2}{|c|}{ LEU06-G01 } \\
\hline Weight of particles: & \multirow{2}{*}{\multicolumn{2}{|c|}{$\frac{4.0109}{3872}$}} & \multicolumn{2}{|c|}{3.9200} & \multicolumn{2}{|c|}{3.8813} \\
\hline Approximate number of particles: & & & \multicolumn{2}{|c|}{3784} & \multicolumn{2}{|c|}{3746} \\
\hline Uncertainty in number of particles: & \multicolumn{2}{|c|}{4} & \multicolumn{2}{|c|}{3} & \multicolumn{2}{|c|}{3} \\
\hline Acid leach sample ID: & U08122301 & U08122901 & U08122302 & U08122902 & U08122303 & U08122903 \\
\hline Radiochemical laboratory analysis number: & $1585-001$ & $1585-004$ & $1585-002$ & $1585-005$ & $1585-003$ & $1585-006$ \\
\hline Weight $U$ in leach $(\mathrm{mg}):$ & 1534 & 0.059 & 1488 & 0.041 & 1485 & 0.027 \\
\hline Uncertainty in weight $U$ in leach (mg): & 6 & 0.00591 & 6 & 0.00406 & 6 & 0.00267 \\
\hline Total weight $\mathrm{U}$ in sample $(\mathrm{mg})$ : & \multicolumn{2}{|c|}{1534} & \multicolumn{2}{|c|}{1488} & \multicolumn{2}{|c|}{1485} \\
\hline Average weight $U$ per particle $(\mathrm{mg}):$ & \multicolumn{2}{|c|}{0.3963} & \multicolumn{2}{|c|}{0.3932} & \multicolumn{2}{|c|}{0.3963} \\
\hline Uncertainty in average weight $U$ per particle $(\mathrm{mg})$ : & \multicolumn{2}{|c|}{0.0016} & \multicolumn{2}{|c|}{0.0016} & \multicolumn{2}{|c|}{0.0016} \\
\hline
\end{tabular}

Mean average uranium loading per particle $(9): 3.953 \mathrm{E}-04$

Standard error in mean average uranium loading per particle $(\mathrm{g}): 1.0 \mathrm{E}-06$

\section{Comments}

Leach 1 was analyzed by Davies-Gray titration method. Leach 2 was analyzed by ICP-MS, due to low U concentration.

Leach 1 was titrated 2 times and averaged.

Davies-Gray Initial known U recovery: $100.30 \%$; final known U recovery $100.50 \%$ Blind titration U recovery $100.13 \%$.

Uncertainty in Davies-Gray $(0.4 \%)$ based on average of measured \% recovery data for LEU06,07,08,09.

Checked against RMAL1585 official results by FCM on 3/06/2009. 


\section{Data Report Form DRF-25: Fuel Compact Mean Uranium Loading}

\begin{tabular}{|r|l|}
\hline Procedure: & AGR-CHAR-DAM-25 Rev. 2 \\
\hline Operator: & Fred Montgomery \\
\hline Compact lot ID: & LEU06-OP1-Z \\
\hline Compact lot description: & AGR-2 UCO Variant fuel, from G73J-14-93074A \\
\hline Filename: & IIImC-aarlAGRIUraniumLoadinalLEU06-OP1-Z_DRF25R2.xIs \\
\hline
\end{tabular}

\begin{tabular}{|c|c|c|c|c|c|c|}
\hline & Sample 1 & Sample 2 & Sample 3 & Sample 4 & Sample 5 & Sample 6 \\
\hline Compact ID number: & 2007 & Z054 & Z064 & $\mathrm{Z107}$ & $\mathrm{Z110}$ & Z135 \\
\hline \multicolumn{7}{|l|}{ First Leach } \\
\hline Sample tube ID number: & U09022701 & U09022702 & U09022703 & U09022704 & U09022705 & U09022706 \\
\hline Radiochemical laboratory analysis number: & $1727-001$ & $1727-002$ & $1727-003$ & $1727-004$ & $1727-005$ & $1727-006$ \\
\hline Weight $U$ in leach $(g)$ : & 1.254 & 1.256 & 1.260 & 1.258 & 1.255 & 1.255 \\
\hline Uncertainty in weight $U$ in leach $(g)$ : & 0.005 & 0.005 & 0.005 & 0.005 & 0.005 & 0.005 \\
\hline \multicolumn{7}{|l|}{ Second Leach } \\
\hline Sample tube ID number: & U09030201 & U09030202 & U09030203 & U09030204 & U09030205 & U09030206 \\
\hline Radiochemical laboratory analysis number: & $1727-007$ & $1727-008$ & $1727-009$ & $1727-010$ & $1727-011$ & $1727-012$ \\
\hline Weight U in leach $(g)$ : & $4.60 \mathrm{E}-05$ & $6.34 E-05$ & $6.70 E-05$ & $3.71 \mathrm{E}-05$ & $5.61 \mathrm{E}-05$ & $3.81 \mathrm{E}-05$ \\
\hline Uncertainty in weight $U$ in leach $(9)$ : & $4.60 \mathrm{E}-06$ & $6.34 \mathrm{E}-06$ & $6.70 \mathrm{E}-06$ & $3.71 \mathrm{E}-06$ & $5.61 \mathrm{E}-06$ & $3.81 \mathrm{E}-06$ \\
\hline Total Measured U & & & & 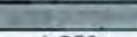 & & \\
\hline Weight $U$ in compact $(g)$ : & 1.254 & 1.256 & 1.260 & 1.258 & 1.255 & 1.255 \\
\hline Uncertainty in weight $U$ in compact ( $q$ ): & 0.005 & 0.005 & 0.005 & 0.005 & 0.005 & 0.005 \\
\hline \multicolumn{3}{|c|}{ Mean uranium loading (gU/compact): } & \multirow{2}{*}{\multicolumn{3}{|c|}{1.256}} & \\
\hline \multicolumn{3}{|c|}{ Standard deviation in mean uranium loading (gU/compact): } & 0.002 & & & \\
\hline
\end{tabular}


Data Report Form DRF-35: Fuel Particle Uranium Loading

\begin{tabular}{|r|l|}
\hline Procedure: & AGR-CHAR-DAM-35 Rev. 0 \\
\hline Operator: & Fred Móntgomery \\
\hline Particle lot ID: & LEU07 \\
\hline Particle lot description: & AGR-2 UCO Baseline, from G73J-14-93072A \\
\hline Filename: & IIMC-agr|AGR\UraniumLoading LLEU07_DRF35R0.xIS \\
\hline
\end{tabular}

Mean average weight per particle $(\mathrm{g}): 1.004 \mathrm{E}-03$

Standard error in mean average weight per particle $(\mathrm{g}): 1.5 \mathrm{E}-06$

\begin{tabular}{|c|c|c|c|c|c|c|}
\hline & \multicolumn{2}{|c|}{ Sample 1 } & \multicolumn{2}{|c|}{ Sample 2} & \multicolumn{2}{|c|}{ Sample 3} \\
\hline & Leach 1 & Leach 2 & Leach 1 & Leach 2 & Leach 1 & Leach 2 \\
\hline Particle sample ID: & \multicolumn{2}{|c|}{ LEU07-E01 } & \multicolumn{2}{|c|}{ LEU07-F01 } & \multicolumn{2}{|c|}{ LEU07-B01 } \\
\hline Weight of particles: & \multicolumn{2}{|c|}{3.8098} & \multicolumn{2}{|c|}{3.9149} & \multicolumn{2}{|c|}{4.0143} \\
\hline Approximate number of particles: & \multicolumn{2}{|c|}{3795} & \multicolumn{2}{|c|}{3899} & \multicolumn{2}{|c|}{3998} \\
\hline Uncertainty in number of particles: & \multicolumn{2}{|c|}{6} & \multicolumn{2}{|c|}{6} & \multicolumn{2}{|c|}{6} \\
\hline Acid leach sample ID: & U09012101 & U09012201 & U09012102 & U09012202 & U09012104 & U09012204 \\
\hline Radiochemical laboratory analysis number: & $1658-001$ & $1658-005$ & $1658-002$ & $1658-006$ & $1658-004$ & $1658-008$ \\
\hline Weight $U$ in leach $(\mathrm{mg}):$ & 1496 & 0.073 & 1529 & 0.360 & 1569 & 0.370 \\
\hline Uncertainty in weight $U$ in leach $(\mathrm{mg})$ : & 6.0 & 0.0073 & 6.1 & 0.036 & 6.3 & 0.037 \\
\hline Total weight $\mathrm{U}$ in sample $(\mathrm{mg}):$ & \multicolumn{2}{|c|}{1496} & \multicolumn{2}{|c|}{1529} & \multicolumn{2}{|c|}{1569} \\
\hline Average weight U per particle $(\mathrm{mg}):$ & \multicolumn{2}{|c|}{0.3943} & \multicolumn{2}{|c|}{0.3922} & \multicolumn{2}{|c|}{0.3924} \\
\hline Uncertainty in average weight U per particle $(\mathrm{mg}):$ & \multicolumn{2}{|c|}{0.0017} & \multicolumn{2}{|c|}{0.0017} & \multicolumn{2}{|c|}{0.0017} \\
\hline
\end{tabular}

Mean average uranium loading per particle $(\mathrm{g}): 3.930 \mathrm{E}-04$

Standard error in mean average uranium loading per particle $(\mathrm{g}): 6.6 \mathrm{E}-07$

\section{Comments}

Leach 1 was analyzed by Davies-Gray titration method. Leach 2 was analyzed by ICP-MS, due to low U concentration.

A small amount of the crushed particle residue was lost during analysis of LEU07-G01 while removing the tape from the spex mill bottle. LEU07-B01 was analyzed in place

of LEU07-G01 as sample \#3.

Particle weight from combined results of 2 independent measurements (W09010801 and W09011403).

Davies-Gray Initial known U recovery: $100.60 \%$; final known U recovery $100.20 \%$ Blind titration U recovery $100.4 \%$.

Uncertainty in Davies-Gray $(0.4 \%)$ based on average of measured \% recovery data for LEU06,07,08,09.

Data checked against official results of analyses for RMAL1658 on 5/26/2009 by FCM 
Data Report Form DRF-25: Fuel Compact Mean Uranium Loading

\begin{tabular}{|r|l|}
\hline Procedure: & AGR-CHAR-DAM-25 Rev. 2 \\
\hline Operator: & Montgomery \\
\hline Compact lot ID: & LEU07-OP1-Z \\
\hline Compact lot description: & AGR-2 UCO Baseline fuel, from G73J-14-93072A \\
\hline Filename: & IIImC-agrIAGRIUraniumLoadinglLEU07-OP1-Z_DRF25R2.xIs \\
\hline
\end{tabular}

\begin{tabular}{|c|c|c|c|c|c|c|}
\hline & Sample 1 & Sample 2 & Sample 3 & Sample 4 & Sample 5 & Sample 6 \\
\hline Compact ID number: & Z057 & $\mathrm{Z} 154$ & Z096 & Z090 & $\mathrm{Z} 120$ & $\mathrm{Z153}$ \\
\hline First Leach & \multicolumn{6}{|c|}{ 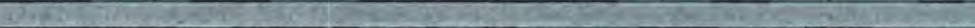 } \\
\hline Sample tube ID number: & U09042001 & U09042002 & U09042003 & U09042004 & U09042005 & U09042006 \\
\hline Radiochemical laboratory analysis number: & $1874-001$ & $1874-002$ & $1874-003$ & $1874-004$ & $1874-005$ & $1874-006$ \\
\hline Weight $U$ in leach $(g)$ : & 1.251 & 1.255 & 1.242 & 1.253 & 1.250 & 1.254 \\
\hline Uncertainty in weight $U$ in leach $(\mathrm{g})$ : & 0.005 & 0.005 & 0.005 & 0.005 & 0.005 & 0.005 \\
\hline \multicolumn{7}{|l|}{ Second Leach } \\
\hline Sample tube ID number: & U09042101 & U09042102 & U09042103 & 009042104 & U09042105 & U09042106 \\
\hline Radiochemical laboratory analysis number: & $1874-007$ & $1874-008$ & $1874-009$ & $1874-010$ & $1874-011$ & $1874-012$ \\
\hline Weight $U$ in leach $(g)$ : & $3.05 \mathrm{E}-04$ & $2.88 \mathrm{E}-04$ & $4.13 E-04$ & $2.94 \mathrm{E}-04$ & $4.86 \mathrm{E}-04$ & $3.80 \mathrm{E}-04$ \\
\hline Uncertainty in weight $U$ in leach $(9)$ : & $3.05 \mathrm{E}-05$ & $2.88 \mathrm{E}-05$ & $4.13 E-05$ & $2.94 \mathrm{E}-05$ & $4.86 \mathrm{E}-05$ & $3.80 \mathrm{E}-05$ \\
\hline Total Measured U & & 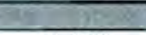 & $\sqrt{1+20}$ & +2 & & \\
\hline Weight $U$ in compact $(\mathrm{g})$ : & 1.251 & 1.255 & 1.242 & 1.253 & 1.251 & 1.254 \\
\hline Uncertainty in weight $\mathrm{U}$ in compact (g): & 0.005 & 0.005 & 0.005 & 0.005 & 0.005 & 0.005 \\
\hline \multicolumn{3}{|c|}{ Mean uranium loading (gU/compact): } & \multirow{2}{*}{\multicolumn{3}{|c|}{$\frac{1.251}{0.005}$}} & \\
\hline \multicolumn{3}{|c|}{ Standard deviation in mean uranium loading (gU/compact): } & & & & \\
\hline
\end{tabular}

Comments

each 1 was analyzed by Davies-Gray titration method. Leach 2 was analyzed by ICP-MS, due to low U concentration.

Davies-Gray Initial known U recovery: $100.25 \%$; final known U recovery $100.18 \%$ Blind titration U recovery $100.39 \%$.

Uncertainty in Davies-Gray $(0.4 \%)$ based on average of measured \% recovery data for LEU06,07,08,09.

wt. \% U235 enrichment: sample $1=14.542$ : sample $2=14.529 ;$ sample $3=14.533$; sample $4=14.529 ;$ sample $5=14.501$; sample $6=14.504$

$U$ data check with official results of analysies for RMAL 1874 by FCM on 5/26/2009

Heed c. montgomery $10-19-2009$

Operator 
Data Report Form DRF-35: Fuel Particle Uranium Loading

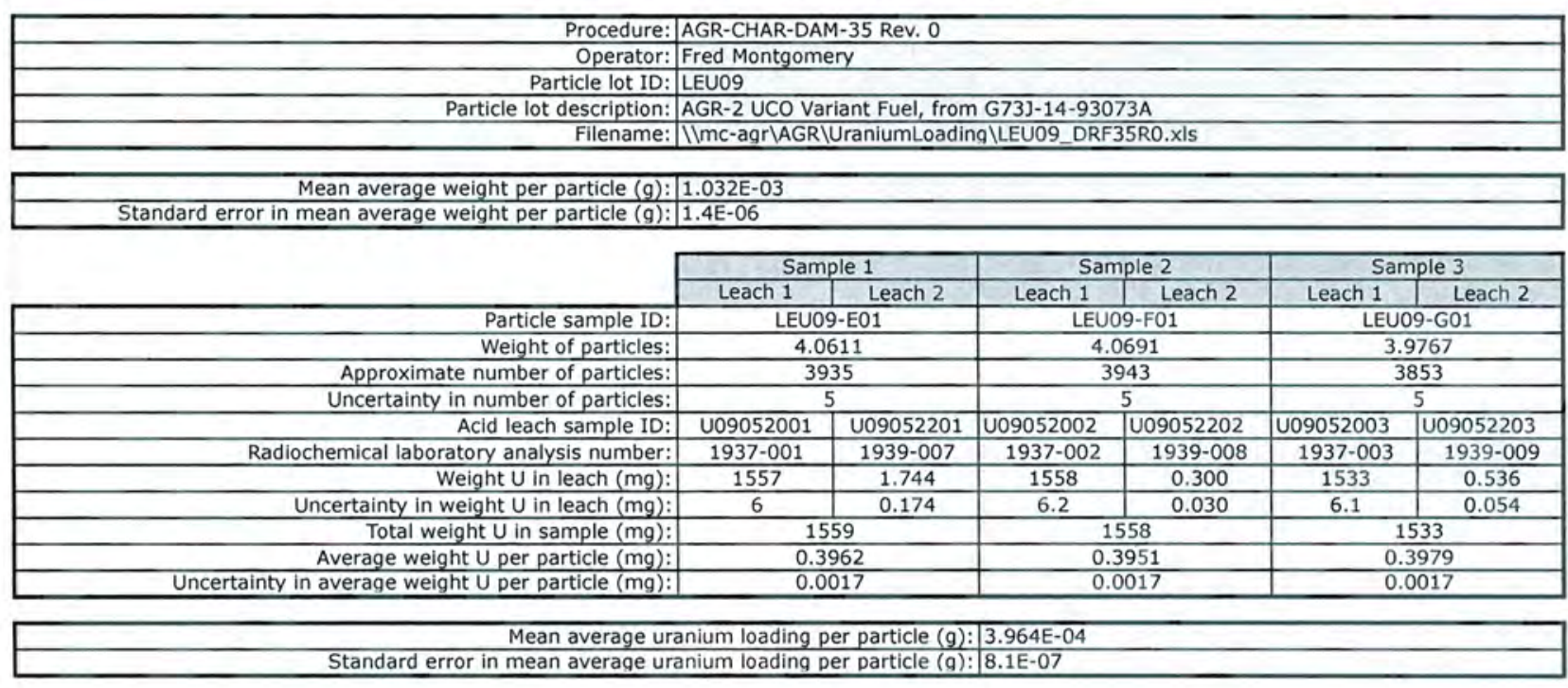

\section{Comments}

Leach 1 was analyzed by Davies-Gray titration method. Leach 2 was analyzed by ICP-MS, due to low U concentration.

Davies-Gray: initial known $U$ recovery $=100.66 \%$; final known $U$ recovery $=100.87 \%$; blind titration $U$ recovery $=100.33 \%$

Uncertainty in Davies-Gray $(0.4 \%)$ based on average of measured \% recovery data for LEU06,07,08,09.

Data checked by FCM against official results of analyses for RMAL1937 on 6/19/2009 and against RMAL1939 on 7/20/2009

\section{Fred C. Montgomery}

$10-19-2009$ 
Data Report Form DRF-25: Fuel Compact Mean Uranium Loading

\begin{tabular}{|r|l|}
\hline Procedure: & AGR-CHAR-DAM-25 Rev. 2 \\
\hline Operator: & Montgomery \\
\hline Compact lot ID: & LEU09-OP2-Z \\
\hline Compact lot description: & AGR-2 UCO Variant Fuel, from G73J-14-93073A \\
\hline Filename: & IImC-agr|AGRIUraniumLoading\LEU09-OP2-Z_DRF25R2.xIS \\
\hline
\end{tabular}

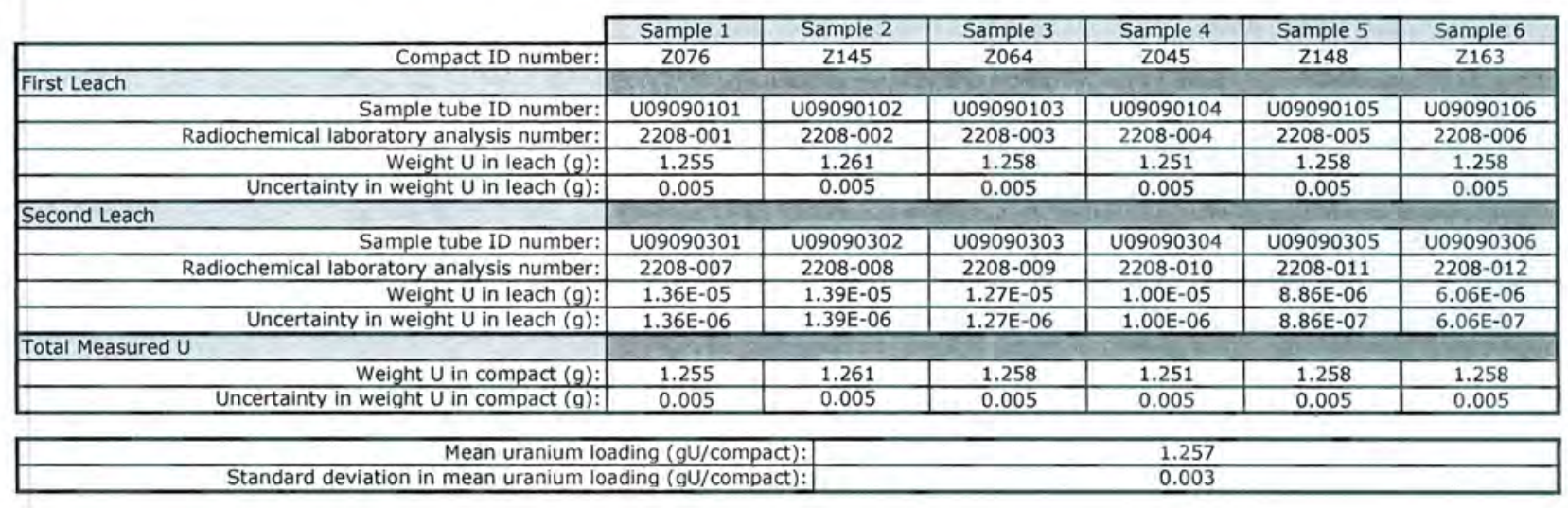

\section{Comments}

Leach 1 was analyzed by Davies-Gray titration method. Leach 2 was analyzed by ICP-MS due to low U concentration.

Davies Gray: Initial known U recovery $=100.37 \%$; Final Known U recovery $=100.13 \%$; Blind titration U recovery $=100.19 \%$

Uncertainty in Davies-Gray $(0.4 \%)$ based on average of measured \% recovery data for LEU06,07,08,09.

Isotopic composition $(w t \%): 234 \mathrm{U}=0.148 \pm 0.001 ; 235 \mathrm{U}=13.993 \pm 0.017 ; 236 \mathrm{U}=0.0504 \pm 0.0004 ; 238 \mathrm{U}=85.809 \pm 0.018$

Data checked against official results of analyses for RMAL2208 by FCM on 11/12/2009

$\frac{\text { Fud C. montgomemy }}{\text { Operator }}$


Data Report Form DRF-35: Fuel Particle Uranium Loading

\begin{tabular}{|c|c|}
\hline Procedure: & AGR-CHAR-DAM- 35 Rev. 0 \\
\hline Operator: & Fred Montgomery \\
\hline Particle lot ID: & LEU11 \\
\hline Particle lot description: & AGR-2 B\&W UO2 Fuel, from G73H-10-93085B \\
\hline Filename: & 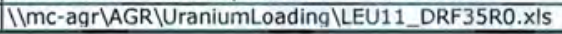 \\
\hline $\begin{array}{l}\text { Mean average weight per particle }(g) \text { : } \\
\text { or in mean average weight per particle }(g) \text { : }\end{array}$ & $\frac{1.462 \mathrm{E}-03}{2.4 \mathrm{E}-06}$ \\
\hline
\end{tabular}

\begin{tabular}{|c|c|c|c|c|c|c|}
\hline & \multicolumn{2}{|c|}{ Sample 1} & \multicolumn{2}{|c|}{ Sample 2} & \multicolumn{2}{|c|}{ Sample 3} \\
\hline & Leach 1 & Leach 2 & Leach 1 & Leach 2 & Leach 1 & Leach 2 \\
\hline Particle sample ID: & \multicolumn{2}{|c|}{ LEU11-E01 } & \multicolumn{2}{|c|}{ LEU11-F01 } & \multicolumn{2}{|c|}{ LEU11-G01 } \\
\hline Weight of particles: & \multirow{2}{*}{\multicolumn{2}{|c|}{$\frac{4.1447}{2834}$}} & \multirow{2}{*}{\multicolumn{2}{|c|}{$\frac{4.1672}{2849}$}} & \multicolumn{2}{|c|}{3.9160} \\
\hline Approximate number of particles: & & & & & \multirow{2}{*}{\multicolumn{2}{|c|}{$\frac{2678}{4}$}} \\
\hline Uncertainty in number of particles: & 5 & 2834 & \multicolumn{2}{|c|}{$\frac{2849}{5}$} & & \\
\hline Acid leach sample ID: & U09060901 & U09061201 & U09060902 & U09061202 & U09060903 & U09061203 \\
\hline Radiochemical laboratory analysis number: & $1998-001$ & $1999-031$ & $1998-002$ & 1999-032 & $1998-003$ & 1999-033 \\
\hline Weight U in leach $(\mathrm{mg}):$ & 1813 & 0.409 & 1816 & 0.620 & 1710 & 0.608 \\
\hline Uncertainty in weight $\mathrm{U}$ in leach $(\mathrm{mg})$ : & 7 & 0.041 & 7 & 0.062 & 7 & 0.061 \\
\hline Total weight $\mathrm{U}$ in sample $(\mathrm{mg}):$ & \multicolumn{2}{|c|}{1813} & \multicolumn{2}{|c|}{1816} & \multicolumn{2}{|c|}{1710} \\
\hline Average weight U per particle $(\mathrm{mg}):$ & \multirow{2}{*}{\multicolumn{2}{|c|}{$\frac{0.6399}{0.0028}$}} & \multirow{2}{*}{\multicolumn{2}{|c|}{$\frac{0.6374}{0.0027}$}} & \multirow{2}{*}{\multicolumn{2}{|c|}{$\begin{array}{l}0.6386 \\
0.0028\end{array}$}} \\
\hline Uncertainty in average weight $U$ per particle $(\mathrm{mg})$ : & & & & & & \\
\hline
\end{tabular}

Mean average uranium loading per particle $(\mathrm{g}): 6.386 \mathrm{E}-04$ Standard error in mean average uranium loading per particle $(\mathrm{g}): 07.0 \mathrm{E}-07$

\section{Comments}

Leach 1 was analyzed by Davies-Gray titration method. Leach 2 was analyzed by ICP-MS, due to low U concentration.

initial known $U$ recovery: $100.50 \%$. Final Known $U$ recovery: $100.32 \%$. Blind titration $U$ recovery: $100.62 \%$

Uncertainty in Davies-Gray $(0.4 \%)$ based on average of measured \% recovery data for LEU06,07,08,09.

Data checked by FCM against official results of analyses for RMAL1998 and RMAL1999 on 7/20/09

\section{Fied c. Montgomery 10-19-2009




\section{Data Report Form DRF-25: Fuel Compact Mean Uranium Loading}

\begin{tabular}{|r|l|}
\hline Procedure: & AGR-CHAR-DAM-25 Rev. 2 \\
\hline Operator: & Montgomery \\
\hline Compact lot ID: & LEU11-OP2-Z \\
\hline Compact lot description: & AGR-2 B\&W UO2 Fuel, from G73H-10-93085B \\
\hline Filename: & IImC-agrVAGRIUranlumLoadinglLEU11-OP2-Z_DRF25R2.xIS \\
\hline
\end{tabular}

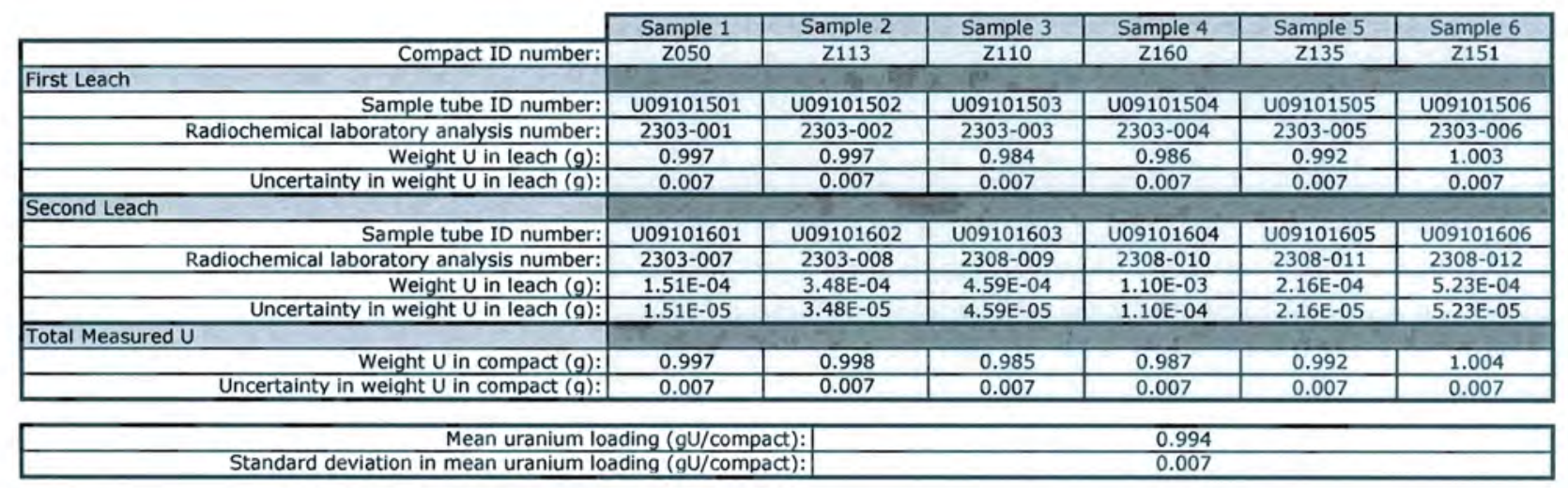

\section{Comments}

Leach 1 was analyzed by Davies-Gray titration method. Leach 2 was analyzed by ICP-MS, due to low U concentration

wt. $\%$ U235 enrichment: sample $1=9.63$; sample $2=9.62$; sample $3=9.62$; sample $4=9.62$; sample $5=9.62 ;$ sample $6=9.62$.

Davies gray: initial known $U$ recovery $=100.48 \%$; final known $U$ recovery $=100.69 \%$; blind titration $U$ recovery $=100.92 \%$

Uncertainty in Davies-Gray $(0.7 \%)$ based on average of measured \% recovery data for this analysis.

Checked against official results of analyses for RMAL2303 by FCM on 11/19/2009

\section{Zed c. monteromeny


Data Report Form DRF-25: Fuel Compact Mean Uranium Loading

\begin{tabular}{|r|l|}
\hline Procedure: & AGR-CHAR-DAM-25 Rev. 2 \\
\hline Operator: & Montgomery \\
\hline Compact lot ID: & LEU11-OP2-Z \\
\hline Compact lot description: & AGR-2 B\&W UO2 Fuel, from G73H-10-93085B \\
\hline Filename: & IImC-agr\AGRIUraniumLoading|LEU11-OP2-Z_Reanalysis_DRF25R2.xIS \\
\hline
\end{tabular}

\begin{tabular}{|c|c|c|c|c|c|c|}
\hline & Sample 1 & Sample 2 & Sample 3 & Sample 4 & Sample 5 & Sample 6 \\
\hline Compact ID number: & $\mathrm{Z} 050$ & Z113 & Z110 & $\mathrm{Z} 160$ & $\mathrm{Z} 135$ & $\mathrm{Z151}$ \\
\hline \multicolumn{7}{|l|}{ First Leach } \\
\hline Sample tube ID number: & U09101501 & U09101502 & U09101503 & U09101504 & U09101505 & U09101506 \\
\hline Radiochemical laboratory analysis number: & $2382-001$ & $2382-002$ & $2382-003$ & $2382-004$ & $2382-005$ & $2382-006$ \\
\hline Weight U in leach $(g):$ & 0.994 & 0.995 & 0.984 & 0.983 & 0.992 & 0.998 \\
\hline Uncertainty in weight $U$ in leach (a): & 0.007 & 0.007 & 0.007 & 0.007 & 0.007 & 0.007 \\
\hline \multicolumn{7}{|l|}{ Second Leach } \\
\hline Sample tube ID number: & U09101601 & 009101602 & U09101603 & U09101604 & U09101605 & U09101606 \\
\hline Radiochemical laboratory analysis number: & $2303-007$ & $2303-008$ & $2308-009$ & $2308-010$ & $2308-011$ & $2308-012$ \\
\hline Weight U in leach $(g)$ : & $1.51 \mathrm{E}-04$ & $3.48 \mathrm{E}-04$ & $4.59 \mathrm{E}-04$ & $1.10 \mathrm{E}-03$ & $2.16 \mathrm{E}-04$ & $5.23 \mathrm{E}-04$ \\
\hline Uncertainty in weight U in leach (g): & $1.51 \mathrm{E}-05$ & $3.48 \mathrm{E}-05$ & $4.59 \mathrm{E}-05$ & $1.10 \mathrm{E}-04$ & $2.16 \mathrm{E}-05$ & $5.23 \mathrm{E}-05$ \\
\hline \multicolumn{7}{|l|}{ Total MeasuredU } \\
\hline Weight $\mathrm{U}$ in compact $(\mathrm{g})$ : & 0.995 & 0.995 & 0.984 & 0.984 & 0.992 & 0.998 \\
\hline Uncertainty in weight $\mathrm{U}$ in compact $(\mathrm{g})$ : & 0.007 & 0.007 & 0.007 & 0.007 & 0.007 & 0.007 \\
\hline \multicolumn{7}{|c|}{ Mean uranium loading $(\mathrm{gU} / \mathrm{com}$} \\
\hline \multicolumn{3}{|c|}{$\begin{array}{l}\text { Mean uranium loading ( } \mathrm{gU} / \mathrm{com} \\
\text { Standard deviation in mean uranium loading }(\mathrm{gU} / \mathrm{com}\end{array}$} & & 0.006 & & \\
\hline
\end{tabular}

Analysis of leach solutions was repeated because of higher than normal uncertaintyin first analysis.

Leach 1 was analyzed by Davies-Gray titration method. D.G. data from 2 nd analysis. Leach 2 was analyzed by ICP-MS, due to low U concentration.

wt. $\%$ U235 from initial Davies-Gray titration samples: sample $1=9.63$; sample $2=9.62$; sample $3=9.62$; sample $4=9.62$; sample $5=9.62 ;$ sample $6=9.62$.

Davies gray: initial known $U$ recovery $=100.49 \%$; final known $U$ recovery $=100.66 \%$; blind titration $U$ recovery $=100.84 \%$

Uncertainty in Davies-Gray $(0.7 \%)$ based on average of measured \% recovery data for this analysis.

Checked against official results of analyses for RMAL2382 by FCM on 11/19/2009

\section{Fied c. Muntgomery

